Probing Ultrafast Dynamics of Bacterial Reaction Centers

\title{
Using Two-Dimensional Electronic Spectroscopy
}

by

Andrew J. Niedringhaus

A dissertation submitted in partial fulfillment

of the requirements for the degree of

Doctor of Philosophy

(Physics)

in the University of Michigan

2017

Doctoral Committee:

Professor Jennifer P. Ogilvie, Chair

Professor Steven T. Cundiff

Professor Eitan Geva

Professor Kevin J. Kubarych

Professor Roseanne J. Sension 
Andrew J. Niedringhaus

aniedrin@umich.edu

ORCID iD: 0000-0002-8385-6706 


\section{ACKNOWLEDGMENTS}

I would like to thank my advisor, Professor Ogilvie, for her guidance and advice throughout my time here at U of M. She has provided interesting, challenging, and important research problems which have pushed me to grow as a scientist. Whenever I felt stuck on a problem, her insight and optimism would always put things in perspective. Thank you to the past group members - Frank, Daniel, Seckin, Jeff, Kristin - for doing most of the legwork in developing the versatile 2D setup I inherited. I would like to thank Veronica for her hard work doing all the sample preps for the experiments presented here, and for all the late nights and early mornings she spent running experiments. To the 2015 TRVS group - Anton, Veronica, Seckin, Libby, Yassel - thank you for going along with my ill-conceived plan to traverse 20 miles through the Porcupine Mountains in one day. It didn't look that far on the map. Thank you to Professors Christine Kirmaier and Dewey Holten at Washington University for providing the BRC samples, and for patiently answering our many questions.

Finally, I want to thank my wife Ellen for her love, support, and encouragement, and for

her patience and understanding through the many late nights and weekends I have spent working on this project. 
TABLE OF CONTENTS

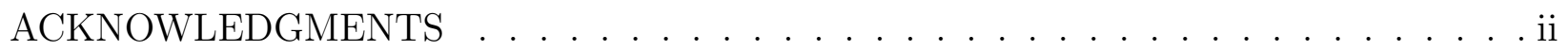

LIST OF FIGURES . . . . . . . . . . . . . . . . . . . . vi

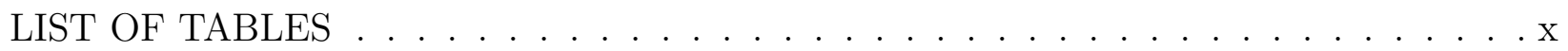

LIST OF ABBREVIATIONS . . . . . . . . . . . . . . . . . . . xi

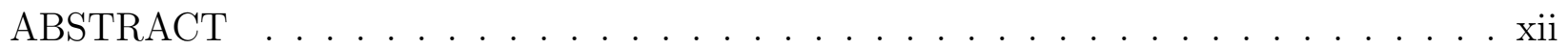

CHAPTER 1 INTRODUCTION $\ldots \ldots \ldots \ldots \ldots \ldots \ldots$

1.1 Bacterial Photosynthesis . . . . . . . . . . . . . . . . . 1

1.2 Ultrafast Energy Transfer and Charge Separation . . . . . . . . . . . . . 6

1.3 Thesis Outline . . . . . . . . . . . . . . . . . . . . . . . . . . . 10

CHAPTER 2 TWO-DIMENSIONAL ELECTRONIC SPECTROSCOPY . . . . . . . 13

2.1 2DES Theory . . . . . . . . . . . . . . . . . . . 14

2.2 Experimental Implementation . . . . . . . . . . . . . . . . . . . 21

2.2 .1 Phase Cycling . . . . . . . . . . . . . . . . . . . . . . 23

2.2 .2 Spectral Interferometry and Phasing . . . . . . . . . . . . . 27

$2.2 .3 \quad$ BRC 2DES Experiments . . . . . . . . . . . . . . . . . . . . 29

2.3 Optical Parametric Amplifiers . . . . . . . . . . . . . . . . . . . . . . . 32

CHAPTER 3 ANALYSIS OF TIME-RESOLVED SPECTRA . . . . . . . . . . . . 38 
3.1 Kinetic Fitting of Transient Spectra . . . . . . . . . . . . . . . . 39

3.1.1 Exponential and Sequential Kinetic Models . . . . . . . . . . . . . . . . 42

3.1.2 Uniqueness of Compartmental Models . . . . . . . . . . . . . . . . . 44

3.1 .3 Temperature-Dependent Kinetic Fitting . . . . . . . . . . . 46

3.1.4 Excitation-Dependent Kinetic Fitting . . . . . . . . . . . . . . 47

3.2 Global Analysis of 2DES Data . . . . . . . . . . . . . . . 48

3.2 .12 D Analog to DADS and SADS . . . . . . . . . . . 48

3.2.2 Excitation-Dependent 2DES Fitting . . . . . . . . . . . . . . . . 49

3.2.3 Simultaneous Fitting of Multiple Data Sets . . . . . . . . . . . . . . 55

3.3 The Magic Angle and Polarization Associated Spectra . . . . . . . . . . . 56 CHAPTER 4 2DES KINETIC FITTING OF BRC MUTANTS . . . . . . . . . . . 61

4.1 Real Absorptive 2DES Data . . . . . . . . . . . . . . . . . . 63

4.1 .1 The $\mathrm{W}(\mathrm{M} 250) \mathrm{V}$ Mutant . . . . . . . . . . . . . . . . . . 63

4.1 .2 The DLL Mutant . . . . . . . . . . . . . . . . . . . 68

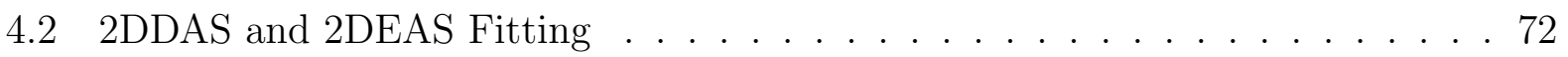

$4.2 .1 \mathrm{~W}(\mathrm{M} 250) \mathrm{V} 2 \mathrm{DDAS}$ Fitting $\ldots \ldots \ldots \ldots \ldots . \ldots \ldots$

$4.2 .2 \mathrm{~W}(\mathrm{M} 250) \mathrm{V}$ 2DEAS Fitting $\ldots \ldots \ldots \ldots \ldots . \ldots . \ldots . \ldots$

4.2 .3 DLL 2DEAS Fitting $\ldots \ldots \ldots \ldots \ldots \ldots \ldots$

4.3 Fitting of Individual Excitation Bands _ . . . . . . . . . . . . 80

4.4 Multi-excitation Global Analysis . . . . . . . . . . . . . . . . . . . . 89

4.4 .1 Optimization Parameters . . . . . . . . . . . . . . . . . . . . 89

4.4 .2 Kinetic model $\# 1 \ldots \ldots$. . . . . . . . . . . . . . . . . . . . 94

$4.4 .3 \quad$ Kinetic model $\# 2 \ldots \ldots$ 
4.5 Discussion . . . . . . . . . . . . . . . . . . 105

CHAPTER 5 CONCLUSION . . . . . . . . . . . . . . . . 107

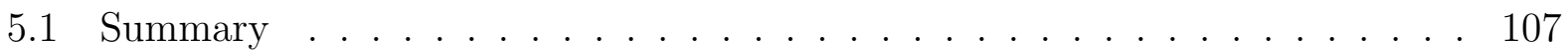

5.2 Future Directions . . . . . . . . . . . . . . . . . 109

REFERENCES . . . . . . . . . . . . . . . . . . . . 112 


\section{LIST OF FIGURES}

Figure 1.1 Structure of the bacterial reaction center from $R$. sphaeroides. P: special pair, B: bacteriochlorophyll a, H: bacteriopheophytin a, Q: ubiquinone. Figure adapted from $[9] . \ldots \ldots$. . . . . . . . . . . . . . . . . 3

Figure 1.2 Linear absorption spectrum of the bacterial reaction center from $R$. capsulatus. The $\mathrm{P}, \mathrm{B}$, and $\mathrm{H}$ absorption bands correspond to the special pair, accessory Bchl, and BPheo, respectively. . . . . . . . . . . . . 3

Figure 1.3 Photocycle of the reaction center for bacterial photosynthesis. The initial photoexcitation and charge separation steps highlighted in red are the focus of this study. Figure adapted from [16] . . . . . . . . . . . . . . 4

Figure 1.4 Energy level diagram indicating the timescales involved for the initial photoinduced reactions in the bacterial reaction center. Figure adapted from $[16] . \ldots \ldots \ldots \ldots \ldots$

Figure 1.5 Branched charge separation pathways from photoexcitation of $\mathrm{B}_{A}$. From left to right: $\mathrm{B}^{*} \rightarrow \mathrm{P}^{*} \rightarrow \mathrm{P}^{+} \mathrm{B}_{A}^{-} \rightarrow \mathrm{P}^{+} \mathrm{H}_{A}^{-} ; \mathrm{B}^{*} \rightarrow \mathrm{P}^{+} \mathrm{B}_{A}^{-} \rightarrow \mathrm{P}^{+} \mathrm{H}_{A}^{-}$; $\mathrm{B}^{*} \rightarrow \mathrm{B}_{A}^{+} \mathrm{H}_{A}^{-} \rightarrow \mathrm{P}^{+} \mathrm{H}_{A}^{-}$. BRC figure adapted from $[2] . \ldots . . . . .8$

Figure 2.1 Pulse sequence used for 2DES experiments. $\tau$ : coherence time, T: population time, t: probe/local oscillator delay (fixed) . . . . . . . . 16

Figure 2.2 Phase-matched four wave mixing signals in the box-CARS geometry. $\vec{k}_{\mathrm{R}}$ and $\vec{k}_{\mathrm{NR}}$ correspond to rephasing and nonrephasing signals, respectively. . . 17

Figure 2.3 Illustration of the 2D spectrum of an inhomogeneously broadened lineshape. (A) Linear absorption spectrum comprised of a Gaussian distribution of narrow Lorentzian peaks. (B) Representative sketch of the associated absorptive $2 \mathrm{D}$ spectrum. . . . . . . . . . . . . . . 19 
Figure 2.4 Features appearing in absorptive 2D spectra for several simple systems. (A) Two uncoupled systems, with excited state absorption. (B) Two weakly coupled two-level systems with downhill energy transfer. (C) Two strongly coupled two-level systems exhibiting excitonic splitting. Figure adapted from [94]. . . . . . . . . . . . . . . . . . . . . . 20

Figure 2.5 Layout of 2DES setup based on the design of Fuller et al. . DOPA: degenerate optical parametric amplifier, BS: beam splitter, CM: chirped mirror, DO: diffractive optic, LO: local oscillator . . . . . . . . . . . . . . . 21

Figure 2.6 Diffractive optic beam geometry used to make box-CARS configuration. Beam blocks are placed in front of the imaging mirror to select the pump, probe and LO beams. A fused silica window is inserted in the LO path to impart a time delay for spectral interferometry. . . . . . . . . . . 22

Figure 2.7 Pulse timing diagrams for the four pump-pair interactions generating signal in the $-\vec{k}_{1}+\vec{k}_{2}+\vec{k}_{3}$ direction. Pulses closer to the center arrive at the sample first. Red and orange circles represent pump pulses with phases $\phi_{1}$ and $\phi_{2}$ respectively, and blue represents the probe pulse. Figure adapted from [95]. . . . . . . . . . . . . . . . . . 23

Figure 2.8 Linear absorption spectra at $298 \mathrm{~K}$ and $77 \mathrm{~K}$, pump and probe spectra used for W(M250)V and DLL 2DES experiments. . . . . . . . . . . . . 32

Figure 2.9 Noncollinear pump, signal, and idler wavevectors . . . . . . . . . . . . 36

Figure 2.10 Schematic of Degenerate OPA design. BS: beam splitter, BBO: $\beta$-barium borate crystal, YAG: yttrium aluminum garnet crystal, PM: off-axis parabolic mirror, DM: dichroic mirror, LPF: long-pass filter $(1 \mu \mathrm{m}), \mathrm{HS}$ : harmonic separater $(400 \mathrm{~nm} / 800 \mathrm{~nm}) \ldots . . \ldots . . \ldots 37$

Figure 3.1 Dipole orientation dependence of the parallel $\left(S_{\|}\right)$, perpendicular $\left(S_{\perp}\right)$, $S_{z}$, and $S_{y}$ signals as a function of angle $\alpha_{i j}$ between state transition dipoles.

Figure 4.1 Linear absorption spectra from W(M250)V and DLL vs wavenumber . . . 64

Figure 4.2 Absorptive 77K 2DES spectra from W(M250)V mutant BRCs with magic angle polarization . . . . . . . . . . . . . . . 65

Figure $4.3 S_{y}$ polarization associated spectra from $\mathrm{W}(\mathrm{M} 250) \mathrm{V}$ at $77 \mathrm{~K} \ldots . . . . .66$

Figure $4.4 S_{z}$ polarization associated spectra from $\mathrm{W}(\mathrm{M} 250) \mathrm{V}$ at $77 \mathrm{~K} \ldots$. . . . . 67 
Figure 4.5 Real absorptive 77K 2DES spectra from DLL mutant BRCs with magic angle polarization . . . . . . . . . . . . . . . . . . 69

Figure $4.6 S_{y}$ polarization associated spectra from DLL mutant at $77 \mathrm{~K} \ldots \ldots$

Figure $4.7 S_{z}$ polarization associated spectra from DLL mutant at $77 \mathrm{~K} \ldots \ldots 71$

Figure 4.8 (A) 4-compartment 2DDAS basis spectra from W(M250)V magic angle data at $77 \mathrm{~K}$. (B) Time-dependent concentrations of the basis spectra, plotted on a split linear/logarithmic scale. . . . . . . . . . . . . . 73

Figure 4.9 (A-D) 2DEAS spectra for $\mathrm{W}(\mathrm{M} 250) \mathrm{V}$ with magic angle polarization using a 4-compartment unbranched sequential model. (E) Time-dependent concentrations. . . . . . . . . . . . . . . 75

Figure 4.10 (A-C) 2DEAS spectra for DLL using a 3-compartment unbranched sequential model. (B) Time-dependent concentrations. . . . . . . . . . 78

Figure 4.11 (A-C) 2DEAS spectra for DLL using a 3-compartment model with the same rates as Figure 4.10 in reverse order. (B) Time-dependent concentrations. . . . . . . . . . . . . . . . . . . 79

Figure 4.12 (A) 77K linear absorption spectrum from W(M250)V, the pump spectrum used for the 2DES experiments, and the four excitation bands selected for kinetic fitting, corresponding to $865 \mathrm{~nm}, 818 \mathrm{~nm}, 796 \mathrm{~nm}$, and $760 \mathrm{~nm}$. (B) Excitation bands projected onto a representative 2D spectrum at $\mathrm{T}=2 \mathrm{ps} \ldots \ldots \ldots \ldots \ldots$. . . . . . . . . . . . . . . . . .

Figure 4.13 SADS derived from cross-sections of W(M250)V 2DES spectra at specific excitation wavelengths. (A-C) The rate constants were fixed at the values reported by Brederode et al.. (D-F) SADS published by Brederode et al. corresponding to similar excitation wavelengths. . . . . . . . . . 82

Figure $4.14 \mathrm{P}^{+} \mathrm{H}_{A}^{-}$SADS from $796 \mathrm{~nm}$ and $818 \mathrm{~nm}$ excitation (blue and red respectively), normalized to the P-band bleaching. Plotted in green is the difference between the two normalized spectra. . . . . . . . . . . . 84

Figure 4.15 Optimized SADS and time constants corresponding to (A-C) of Figure 4.1384

Figure 4.16 (A) 4-compartment SADS calculated from 760nm excitation of W(M250)V. (B) Measured spectra (black) and SADS fit (red) from slices of the 2DES spectrum at $760 \mathrm{~nm}$ excitation and several population times. . 85

Figure 4.17 2-compartment SADS calculated from DLL 2D spectra at specific

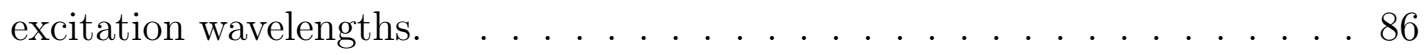


Figure 4.18 Measured spectra (black) and SADS fit (red) from the 2-compartment models shown in Figure $4.17 \ldots$. . . . . . . . . . . . . . 87

Figure $4.1977 \mathrm{~K}$ linear absorption spectrum of $\mathrm{W}(\mathrm{M} 250) \mathrm{V}$ (red) and the resulting fit using six peaks with dipole strengths taken from . . . . . . . . . . 90

Figure 4.20 Reaction scheme of model \#1 used for 2D multi-excitation global fitting . . 94

Figure 4.21 Basis spectra from the global analysis of W(M250)V. The spectra plotted in red were constrained to match the SADS from Figure 4.13D-E. The black basis spectra were calculated by linear least squares. . . . . . . . . . 95

Figure 4.22 Comparison of measured 2D spectra (left) and 2D spectra reconstructed from the global fit (right) at population times of 31.4fs (top) and 356fs (bottom) . . . . . . . . . . . . . . . . . 97

Figure 4.23 Comparison of measured 2D spectra (left) and 2D spectra reconstructed from the global fit (right) at population times of $2 \mathrm{ps}$ (top) and $45 \mathrm{ps}$

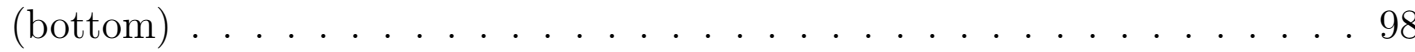

Figure 4.24 Comparison between W(M250)V 2DES data (black) and reconstructed data from global fit (red) at several excitation wavelengths and population times. . . . . . . . . . . . . . . . . . . . . 99

Figure 4.25 Time-dependent concentrations from global fit at several excitation wavelengths representing selective excitation of different mixtures of chromophores in the $\mathrm{P}, \mathrm{B}$ and $\mathrm{H}$ absorption bands. . . . . . . . . . . 100

Figure 4.26 (A) Basis spectra from 2D global fit with the reaction scheme in Figure 4.20. Red spectra were constrained to match the SADS from Figure 4.13D-E, and the black spectrum was calculated by linear least squares. (B) Measured 2D spectrum at 31fs. (C) 2D spectrum reconstructed using the basis spectra from (A) . . . . . . . . . . . 101

Figure 4.27 (A) 9-state branched reaction scheme defining kinetic model \#2. (B) Optimized basis spectra, with red/black being constrained/unconstrained as in model 1. (C) Measured spectra (black) vs fit (red) at several population times and excitation wavelengths. . . . . . . . . 103 


\section{LIST OF TABLES}

Table 4.1 Fitting parameters used for W(M250)V 77K linear absorption spectrum . . 91

Table 4.2 Optimized time constants for the kinetic model depicted in Figure 4.20 . . 96

Table 4.3 Optimized time constants for the second kinetic model, depicted in Figure 4.27 . . . . . . . . . . . . . . . . . . . . . . . . 102 


\section{LIST OF ABBREVIATIONS}

$\beta$-Barium Borate . . . . . . . . . . . . . . . . . BBO

Bacterial Reaction Center . . . . . . . . . . . . . . . . . . . . BRC

Decay Associated Difference Spectrum . . . . . . . . . . . . . . . DADS

Degenerate Optical Parametric Amplifier/Amplification . . . . . . . . . . . . . DOPA

Excited State Absorption _. . . . . . . . . . . . . . ESA

Ground State Bleach . . . . . . . . . . . . . . . . . . GSB

Group Delay Dispersion . . . . . . . . . . . . . . . G GDD

Noncollinear Optical Parametric Amplifier/Amplification . . . . . . . . . . . NOPA

Optical Parametric Amplifier/Amplification . . . . . . . . . . . . . . OPA

Second-Harmonic Generation . . . . . . . . . . . . . . . . SHG

Species Associated Difference Spectrum _. . . . . . . . . . . . . SADS

Stimulated Emission . . . . . . . . . . . . . . . . . SE

Transient Absorption . . . . . . . . . . . . . . . . . TA

Two-Dimensional Decay Associated Spectrum . . . . . . . . . . . . 2DDAS

Two-Dimensional Electronic Spectroscopy . . . . . . . . . . . . . . 2DES

Two-Dimensional Evolution Associated Spectrum . . . . . . . . . . . 2DEAS 


\begin{abstract}
In the initial steps of photosynthesis, solar energy is converted to stable charge separated states with high efficiency. Understanding the relationship between structure and function in the photosynthetic reaction centers where these conversion steps take place could guide the development of more efficient artificial light harvesting systems. Reaction centers are complicated pigment-protein complexes with multiple spectrally overlapped absorption bands, making interpretation of spectroscopic data challenging. The sub-picosecond time scales involved in the energy transfer and charge separation processes present another challenge. Two-dimensional electronic spectroscopy (2DES) has proven to be a powerful tool for disentangling features in spectrally congested systems like reaction centers by resolving the optical response with respect to excitation and detection frequencies. 2DES also obtains the excitation frequency dependence without sacrificing time resolution, which is necessary to resolve energy transfer processes in reaction centers occurring on time scales faster than $100 f s$.

We perform 2DES on bacterial reaction centers (BRCs) from the purple bacterium Rhodobacter capsulatus, using a degenerate optical parametric amplifier producing $12 \mathrm{fs}$ pulses with bandwidth spanning the broad near-IR absorption bands of the BRC. The 2D spectra are analyzed using several global analysis methods to extract the underlying energy transfer and charge separation kinetics, and we compare the results to published transient absorption studies on BRCs. Commonly used 2DES global analysis techniques proved inadequate for resolving specific branched and parallel reaction mechanisms. We developed an improved
\end{abstract}


2D kinetic fitting approach which employs a common set of basis spectra for all excitation frequencies, and uses information from the linear absorption spectrum and BRC structure to model the excitation frequency dependence of the $2 \mathrm{D}$ spectrum. Using the improved fitting method, we show that the entire time-dependent 2D spectrum is well-represented by a sequential reaction scheme with a single charge-separation pathway. We tested several proposed alternative reaction schemes involving branched charge separation pathways, and did not find compelling evidence from our data that favors a particular branched model. Based on this analysis, we conclude that our data supports the simpler, single pathway charge separation model. 


\section{CHAPTER 1}

\section{INTRODUCTION}

Photosynthesis, the process by which sunlight is converted into chemical energy, is arguably the most important chemical reaction required to sustain the abundance of life on Earth. In addition to its ubiquitous role in supporting life, photosynthesis also offers examples of well-optimized systems which efficiently convert photoexcitation into stable chargeseparated states. A better understanding of the functionality of this process could potentially

lead to insights guiding the development of artificial light harvesting devices [1, 2]. Despite decades of studies characterizing the structure and photoexcitation dynamics of the reaction centers where this charge separation occurs, there remain many open questions regarding the relationship between the structure of RCs and the functionality of the system as a whole $[3-5]$.

\subsection{Bacterial Photosynthesis}

Photosynthesis begins with the absorption of photons by light-harvesting antenna complexes. These pigment-protein complexes generally absorb over a wide range of wavelengths, and act as an energy funnel to transfer excitation energy from each pigment, through a sequence of successively lower-energy states, to the reaction center [6]. There, the energy is transferred to a strongly-coupled pair of pigments which donate an electron to a neighboring acceptor. Subsequent electron transfer reactions out-compete charge recombination, resulting in the formation of a stable charge-separated state less than 1ns after the photoexcitation, with a quantum efficiency $>95 \%$ [6-8]. This charge separation is used to create a potential gradient across the photosynthetic membrane, which provides the energy that 
drives the formation of adenosine triphosphate (ATP). The composition, structure, and function of antenna complexes vary substantially between different photosyntheic organisms, as do the methods of chemically storing the energy. The scope of this thesis will be limited to anoxygenic photosynthesis in purple bacteria, although there are many parallels between the bacterial reaction center (BRC) and the photosystem II (PSII) reaction center found in plants [9].

The first measurement of the structure of the BRC was performed in 1984 by x-ray analysis of BRC crystals from Rhodopseudomonas viridis [10, 11]. Soon thereafter, the crystal structure of Rhodobacter sphaeroides was measured with increasingly fine resolution down to $2.3 \AA$ [12-15]. Both are comprised of four bacteriochlorophyll (BChl) pigments, two bacteriopheophytins (BPheo), two quinones, a carotenoid, and one $\mathrm{Fe}^{2+}$ ion [6]. BRCs of $R$. viridis contain bacteriochlorophyll b (BChl b) and bacteriopheophytin b (BPheo b) pigment, absorbing between 770 and 1000nm, while BRCs of $R$. sphaeroides and most other purple bacteria contain Bchl a and BPheo a, and absorb in the 750-900nm region [4].

Figure 1.1 shows the structure of the BRC from $R$. sphaeroides. It is arranged in two branches, joined by a closely spaced, excitonically coupled pair of Bchl called the special pair. Most of the oscillator strength of the special pair is in the lower excitonic state $\mathrm{P}_{-}$, which accounts for the low-energy P-band in the absorption spectrum, near 860nm at room temperature. Nearest the special pair are the "accessory" or monomeric bacteriochlorophylls on the A- and B-branches, with overlapping absorption peaks at 800nm corresponding to the Bchl Qy transition. Next are the two bacteriopheophytins, which account for the $\mathrm{H}$ absorption band at 760nm, and a quinone terminates each branch. These cofactors are held in place by a polypeptide scaffolding structure comprised of three subunits designated L, M, and $\mathrm{H}[3,6]$. The $\mathrm{A}$ - and $\mathrm{B}$-branches are associated with the $\mathrm{L}$ and $\mathrm{M}$ protein subunits, respectively, and the cofactors are sometimes labeled by this convention in the literature.

The photocycle of the bacterial reaction center is depicted in Figure 1.3. The reaction is initiated by the absorption of a photon, either by an antenna complex or direct absorption by 


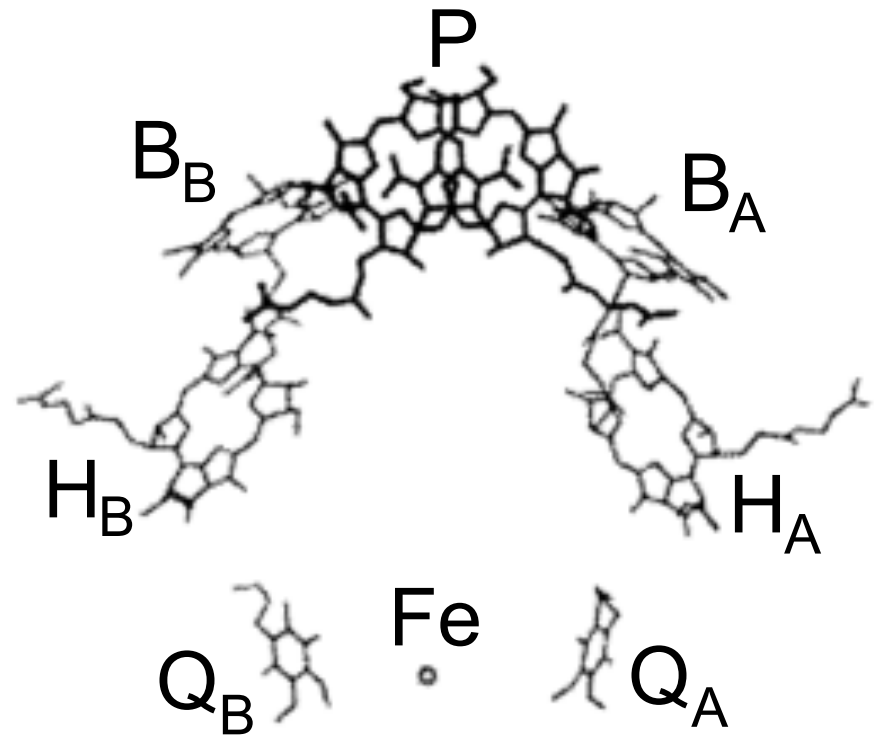

Figure 1.1: Structure of the bacterial reaction center from $R$. sphaeroides. P: special pair, B: bacteriochlorophyll a, H: bacteriopheophytin a, Q: ubiquinone. Figure adapted from [9].

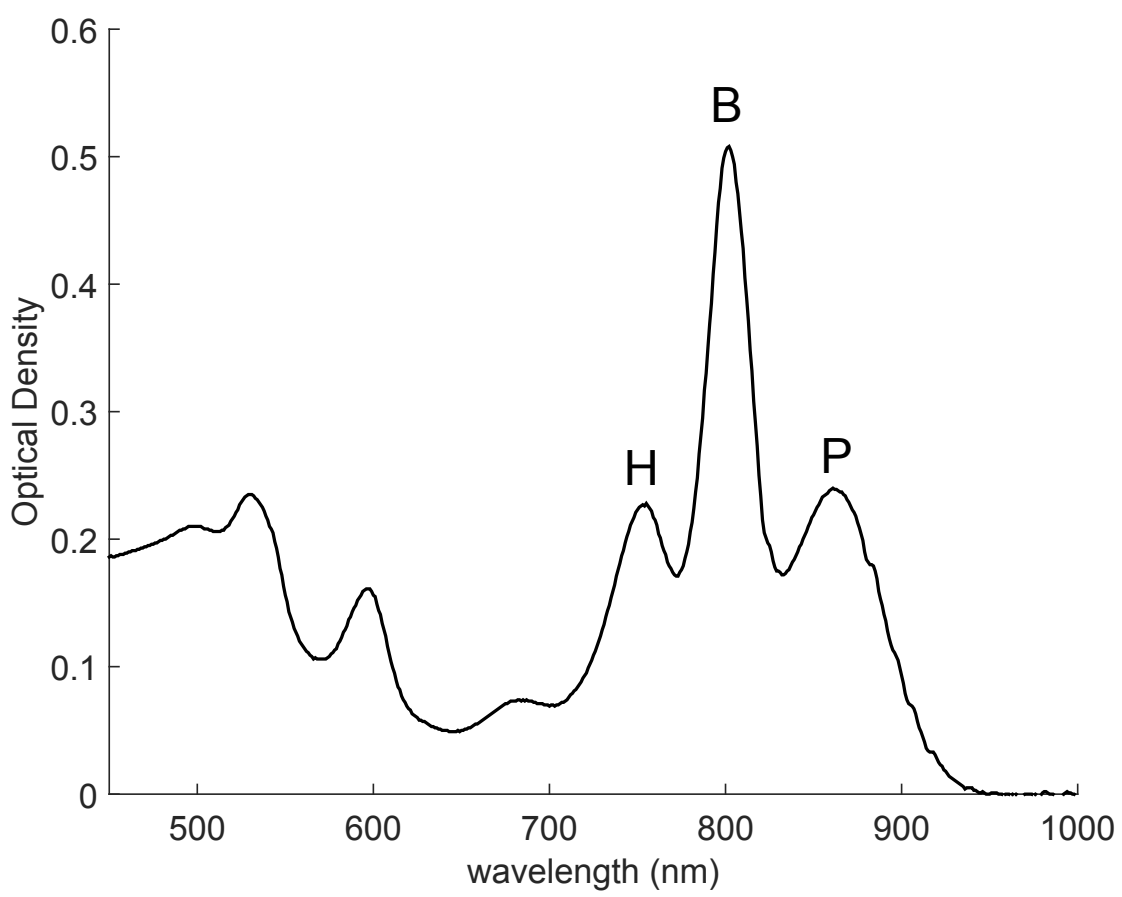

Figure 1.2: Linear absorption spectrum of the bacterial reaction center from $R$. capsulatus. The $\mathrm{P}, \mathrm{B}$, and $\mathrm{H}$ absorption bands correspond to the special pair, accessory Bchl, and BPheo, respectively. 
the BRC. The excitation energy is transferred to the special pair, which donates an electron to the A-branch BPheo, $\mathrm{H}_{A}$. These first two steps of the reaction, highlighted in red in Figure 1.3, are the focus of this thesis. After reducing $\mathrm{H}_{A}$, the electron transfer proceeds to $\mathrm{Q}_{A}$, then across to $\mathrm{Q}_{B}$. A second photoexcitation leads to formation of a doubly reduced $\mathrm{Q}_{B}$, which combines with two protons from the cytoplasm to form a quinol. The quinol is released and replaced by an oxidized quinone, returning the reaction center to its initial state to repeat the process. The quinol formed by this process is then reoxidized and releases the protons to the periplasm, so that the net effect of the reaction is to pump protons from the cytoplasm to periplasm, making the transmembrane potential gradient used for ATP production [6].

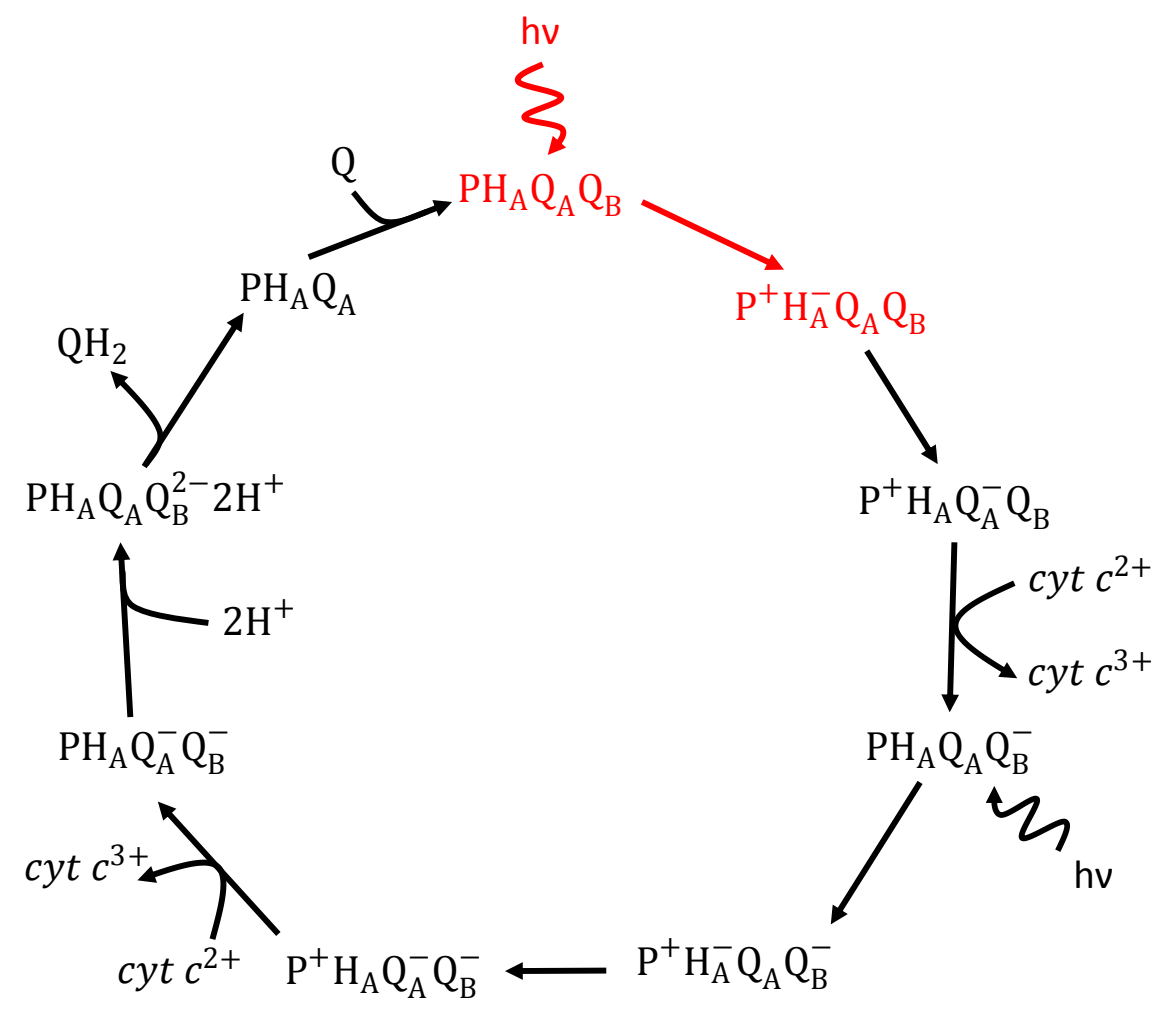

Figure 1.3: Photocycle of the reaction center for bacterial photosynthesis. The initial photoexcitation and charge separation steps highlighted in red are the focus of this study. Figure adapted from [16].

Despite the two branches of the BRC being nearly symmetric, more than $99 \%$ of the electron transfer occurs on the A-branch [4]. The reason for this unidirectionality is not 


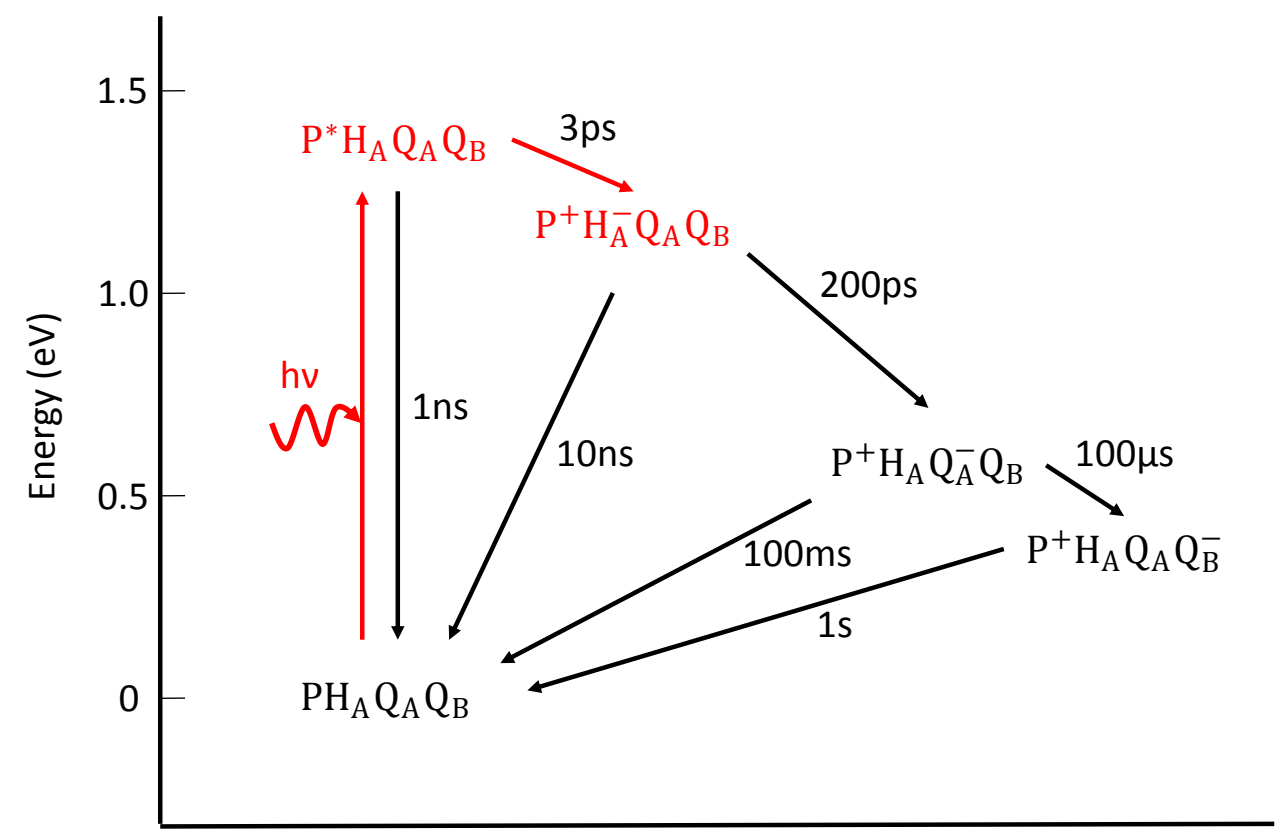

Figure 1.4: Energy level diagram indicating the timescales involved for the initial photoinduced reactions in the bacterial reaction center. Figure adapted from [16].

fully understood [5]. The distances between the Bchl and BPheo are slightly larger on the B-branch than the A-branch [10, 11], resulting in slower $\mathrm{H}_{B} \rightarrow \mathrm{B}_{B}$ energy transfer [16] that may contribute to the preferential A-branch electron transfer. There are also subtle differences in the protein environment between the two branches which could favor one branch over the other [17-19]. Parson et al. showed from calculations based on the crystal structure of $R$. viridis that electrostatic interaction between the special pair and the protein environment favors oxidation of $\mathrm{P}_{B}$ (the special pair Bchl on the B-branch) over $\mathrm{P}_{A}$ [20], suggesting that the protein structure plays a significant role in the reaction kinetics. B-side electron transfer has been demonstrated in a mutant BRC with targeted changes to the Msubunit polypeptide structure [21], demonstrating the importance of the protein structure in determining the free energies of the charge separated states. Another mutant BRC in which the B-branch Bchl is replaced with BPheo has also been shown to exhibit B-side electron transfer [22]. Several groups have since studied B-side electron transfer in various BRC mutants from R. sphaeroides and R. capsulatus [23-26]. 


\subsection{Ultrafast Energy Transfer and Charge Separation}

The initial photo-induced charge separation in BRCs has been studied extensively using time-resolved spectroscopy. Due to the ultrafast time-scales involved, resolving the reaction kinetics requires the use of sub-picosecond laser pulses, with a pump pulse initially exciting the sample, and a time-delayed probe pulse interrogating the time-dependent changes in the optical properties of the sample induced by the excitation. Ultrafast transient absorption and time-resolved fluorescence experiments under a wide variety of excitation conditions, detection wavelengths, and temperatures have been performed on BRCs from R. sphaeroides $[18,19,27-39], R$. viridis $[27,39]$, and $R$. capsulatus [17, 40, 41]. The following is an overview of ultrafast spectroscopic measurements on BRCs. This is by no means an exhaustive review of the photophysics of BRCs, as entire textbooks have been written on the subject (see for example [3, 4]; for shorter review papers, see [5, 9, 42-44]).

Early transient absorption studies resolved the formation of $\mathrm{P}^{+} \mathrm{H}_{A}^{-}$within 2-5ps of photoexcitation depending on the species and strain of the $\mathrm{RC}[27,36,40]$, with a debate as to whether the charge separation mechanism included an intermediate state involving the accessory bacteriochlorophyll $\mathrm{B}_{A}$ or efficient super-exchange from $\mathrm{P}^{*}$ directly to $\mathrm{P}^{+} \mathrm{H}_{A}^{-}[17-$ 19, 37, 38, 45-47]. There is now a general consensus that photoexcitation of the special pair produces $\mathrm{P}^{+} \mathrm{H}_{A}^{-}$through the two-step sequential reaction $\mathrm{P}^{*} \rightarrow \mathrm{P}^{+} \mathrm{B}_{A}^{-} \rightarrow \mathrm{P}^{+} \mathrm{H}_{A}^{-}$, with the first step proceeding with a 2 ps time constant, and the second with $0.9 \mathrm{ps}[5,18,35,38,48-$ $50]$.

However, several studies present evidence of alternative charge separation pathways not involving excitation of the special pair, such as $\mathrm{B}_{A}^{*} \rightarrow \mathrm{B}_{A}^{+} \mathrm{H}_{A}^{-}[46,51,52,52-54]$. Calculation of electronic coupling between the excitonic and charge-transfer states based on the crystal structure of $R$. viridis suggest that a $\mathrm{B}_{A}^{+} \mathrm{H}_{A}^{-}$intermediate might be more energetically favorable than $\mathrm{P}^{+} \mathrm{B}_{A}^{-}[45,47]$. Zhou and Boxer performed higher-order Stark spectroscopy on a number of BRC mutants which revealed an intermediate charge separated species involving both $\mathrm{B}_{A}$ and $\mathrm{H}_{A}$, taken as evidence of a $\mathrm{B}_{A}^{*} \rightarrow \mathrm{B}_{A}^{+} \mathrm{H}_{A}^{-}$electron transfer pathway 
$[55,56]$. Further evidence of an alternative pathway to $\mathrm{P}^{+} \mathrm{H}_{A}^{-}$not involving $\mathrm{P}^{*}$ was found in the YM210W mutant of $R$. sphaeroides by comparing the fluorescence excitation spectra of $\mathrm{P}^{*}$ and $\mathrm{P}^{+} \mathrm{Q}_{A}^{-}$[34]. Huang et al. also found evidence of a $\mathrm{B}_{A}^{*} \rightarrow \mathrm{B}_{A}^{+} \mathrm{H}_{A}^{-}$pathway in $R$. sphaeroides using polarization-selective transient absorption on BRC crystals [57].

Another proposed alternative charge separation pathway involves the formation of $\mathrm{P}^{+} \mathrm{B}_{A}^{-}$ directly from $\mathrm{B}^{*}$, such that $\mathrm{B}_{A}$ transfers a hole to $\mathrm{P}$ rather than the two step $\mathrm{B}_{A}^{*} \rightarrow \mathrm{P}^{*} \rightarrow$ $\mathrm{P}^{+} \mathrm{B}_{A}^{-}$sequence $[51,54]$. van Brederode et al. show evidence of this pathway in YM210 BRC mutants with slowed electron transfer from $\mathrm{P}^{*}[35,53]$. They even suggest a three-way branched reaction in YM210W with approximately a 4:2:1 ratio of $\mathrm{P}^{*}, \mathrm{P}^{+} \mathrm{B}_{A}^{-}$, and $\mathrm{B}_{A}^{+} \mathrm{H}_{A}^{-}$ formed upon excitation of $\mathrm{B}_{A}$ [53]. Figure 1.5 depicts the various proposed charge separation pathways proceeding from photoexcitation of $\mathrm{B}_{A}$.

A number of additional methods beyond probing the Qy band with transient absorption meausurements have proven useful for isolating kinetics and spectroscopic signatures from specific pathways in BRCs.

- Mutant BRCs. One method of determining the functionality of specific components within the complicated, spectrally congested BRC is to make targeted structural changes to the system with point mutations, and infer information from differences in the measured time scales and spectral features in the kinetics [17, 29, 29, 32, 34, 40, 55, 58]. The M182HL mutant of $R$. sphaeroides, in which the B-branch accessory $\mathrm{Bchl} \mathrm{a}$ is replaced with BPheo a, has been studied in relation to the wild-type RC to isolate the energy transfer kinetics of the A-branch [32]. It is generally not possible to distinguish transient absorption signals from the two pathways since the $\mathrm{B}_{A}$ and $\mathrm{B}_{B}$ absorption bands closely overlap. A study on the heterodimer mutant (M)H202L, in which the Mg atom is removed from one of the special pair bacteriochlorophylls, demonstrated that the energy transfer rates to the special pair can be perturbed such that $\mathrm{B}_{A} \rightarrow \mathrm{P}$ and $\mathrm{B}_{B} \rightarrow \mathrm{P}$ proceed at much different rates [58]. The $\beta$ mutant $(\mathrm{M}) \mathrm{L} 214 \mathrm{H}$, which replaces $\mathrm{H}_{A}$ with a bacteriochlorophyll $\beta_{A}$, has well-separated $\mathrm{H}_{B}$ and $\beta_{A}$ ab- 


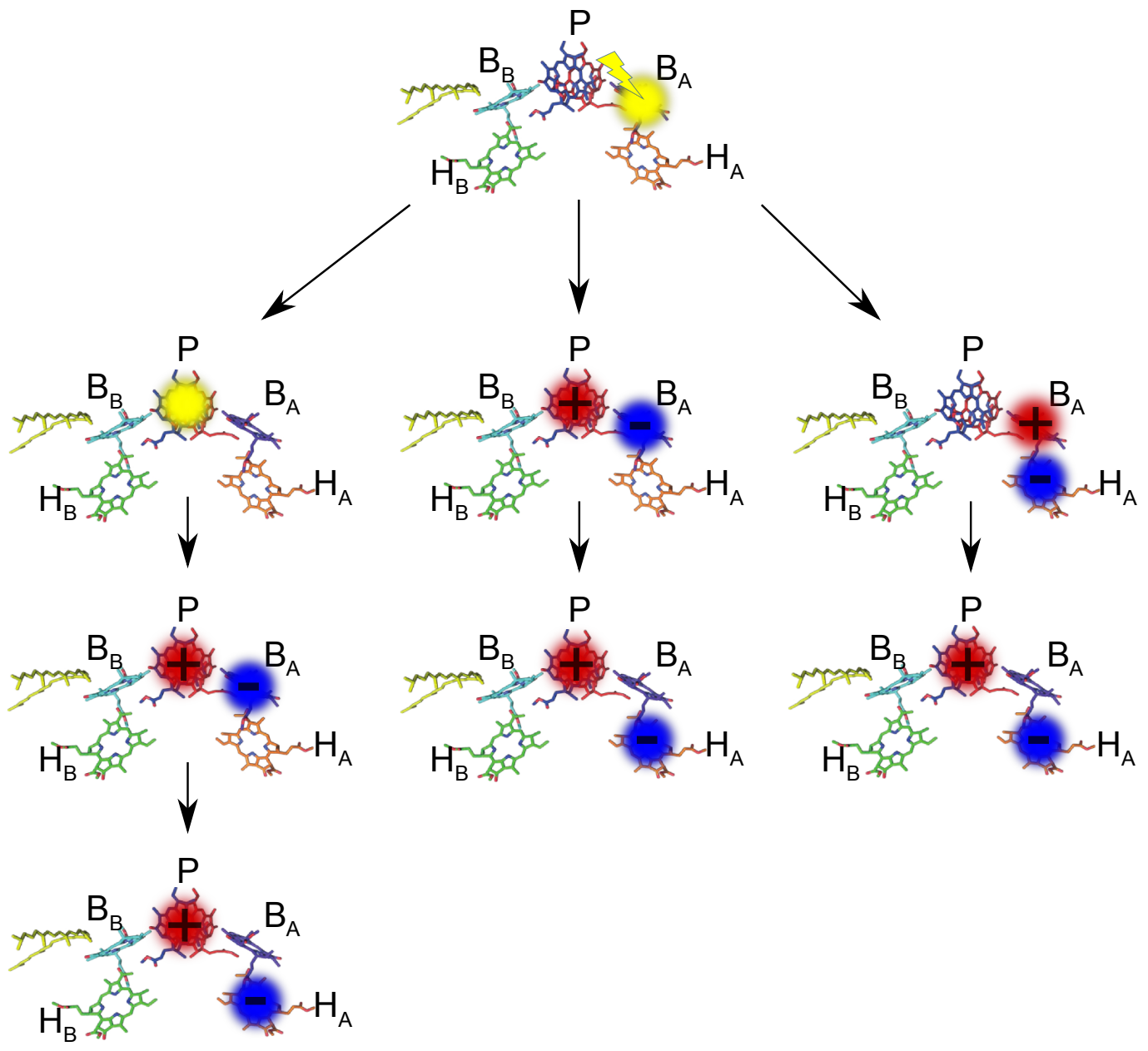

Figure 1.5: Branched charge separation pathways from photoexcitation of $\mathrm{B}_{A}$. From left to right: $\mathrm{B}^{*} \rightarrow \mathrm{P}^{*} \rightarrow \mathrm{P}^{+} \mathrm{B}_{A}^{-} \rightarrow \mathrm{P}^{+} \mathrm{H}_{A}^{-} ; \mathrm{B}^{*} \rightarrow \mathrm{P}^{+} \mathrm{B}_{A}^{-} \rightarrow \mathrm{P}^{+} \mathrm{H}_{A}^{-} ; \mathrm{B}^{*} \rightarrow \mathrm{B}_{A}^{+} \mathrm{H}_{A}^{-} \rightarrow \mathrm{P}^{+} \mathrm{H}_{A}^{-}$. BRC figure adapted from [2]. 
sorption bands which can be selectively excited, revealing similar time constants of 300fs and 239fs for $\mathrm{H}_{B} \rightarrow \mathrm{P}$ and $\beta_{A} \rightarrow \mathrm{P}$ energy transfer, respectively [33]. The $\mathrm{D}_{\mathrm{LL}}$ mutant, which lacks the A-branch BPheo [59], cannot form $\mathrm{P}^{+} \mathrm{H}_{A}^{-}$, allowing for direct measurements of $\mathrm{P}^{*}$ and its decay rate in the absence of charge separation [36, 41]. Pump-probe experiments on the YM210W and YM210L mutants of $R$. sphaeroides, which exhibit very slow, 100 ps charge separation from $\mathrm{P}^{*}$, have successfully isolated charge separation pathways from $\mathrm{B}_{A}$ excitation which do not involve $\mathrm{P}^{*}[34,35,52,53]$.

- Anion absorption bands. Bchl a has anion absorption bands near $650 \mathrm{~nm}$ and $1020 \mathrm{~nm}$ which can be probed to reveal more direct signatures of the intermediate $\mathrm{P}^{+} \mathrm{B}_{A}^{-}$state $[5,38,43,60]$. BPheo also has an anion absorption band near $650 \mathrm{~nm}$ which indicates formation of $\mathrm{P}^{+} \mathrm{H}_{A}^{-}$or $\mathrm{B}^{+} \mathrm{H}_{A}^{-}[5,57,60]$.

- Polarization control/anisotropy. The polarization dependence of the optical response can be used to isolate signals originating from species with different transition dipole directions [28, 31-33, 38, 58, 61]. For example, pump-probe anisotropy measurements, with an 800nm pump exciting both the accessory Bchl $\mathrm{B}_{A}$ and upper excitonic state $\mathrm{P}^{+}$, were compared to theoretical anisotropy calculations based on the crystal structure to show that the $\mathrm{P}^{+}$state is mixed with the excited state of $\mathrm{B}_{A}$ [31], explaining the extremely fast $\tilde{1} 00 \mathrm{fs} \mathrm{B}_{A}^{*} \rightarrow \mathrm{P}^{*}$ energy transfer. Polarization selectivity was also a key component in the pump-probe measurements of BRC crystals by Huang et al. [57], allowing them to selectively excite specific cofactors of the BRC with unprecedented precision.

- Temperature Dependence. The rate of formation of the $\mathrm{P}^{+} \mathrm{H}_{A}^{-}$state has also been shown to vary with temperature $[17,27,38,62]$. Chan et al. [38] concluded from temperaturedependent pump-probe experiments spanning the Bchl a Qx and anion bands that the reaction is dominated by a sequential mechanism involving the $\mathrm{P}^{+} \mathrm{B}_{A}^{-}$intermediate at room temperature, while undergoing a one-step superexchange to $\mathrm{P}^{+} \mathrm{H}_{A}^{-}$at $22 \mathrm{~K}$. 
Energy transfer rates to $\mathrm{P}^{*}$ also have significant temperature-dependence $[16,32,33$, $54,58,61]$. Given a model for the temperature dependence of the transition rates, using the Arrhenius equation or Marcus equation [63] for instance, adds an additional dimension which may be used in a global analysis to extract kinetics [64] (see Section $3.1 .3)$.

\subsection{Thesis Outline}

In the past two decades, the technique of two-dimensional electronic spectroscopy (2DES), which measures the excitation frequency dependence of the detected time-resolved signal, has been successfully applied to study a variety of photosynthetic systems [65-71]. By spreading the frequency dependence of the optical response onto two axes, 2DES reduces the spectral congestion which makes interpretation of transient absorption experiments so challenging for complicated, multi-chromophoric systems such as photosynthetic reaction centers. 2DES also distinguishes homogeneous and inhomogeneous linewidths, which broadband pumpprobe cannot resolve. Using the wealth of information available from $2 \mathrm{D}$ spectroscopy will provide a clearer picture of both the energy transfer and the charge separation processes occurring in the bacterial reaction center of Rhodobacter capsulatus. Specifically, we aim to address the following:

1. Use the entire information content of the 2DES spectrum to quantitatively distinguish between the different charge separation pathways depicted in Figure 1.5. Much of the evidence presented in support of these proposed parallel kinetic reactions involves global analysis of transient absorption data, which is known to produce non-unique results which are difficult to replicate [72-74]. Global fitting of 2D spectra can, in principle, remove this ambiguity and uniquely identify a kinetic model [75].

2. Better resolve the energy transfer rates between specific chromophores. 2DES acquires the excitation dependence obtained from narrow bandwidth pump-probe measurements, while retaining the time resolution imparted by transform-limited broad 
bandwidth pump and probe pulses. The 10-12fs pulses used in our 2DES setup are several times shorter than those used in previous transient absorption experiments measuring energy transfer rates $[32,33,54,58,61]$.

3. Determine what role inhomogeneity plays in the reaction kinetics. Most global and target analyses of BRC kinetics assume a single effective rate constant for each reaction, while multi-exponential fits of pump-probe kinetic data suggest a distribution of rates $[17,29,76,77]$. This can been modeled by a rate constant $k(\Delta G)$ with a distribution of free energies and thermal fluctuations which yields the averaged, apparent rates of the system $[62,78]$. 2DES is well-suited to address this question by resolving inhomogeneous lineshapes and the excitation frequency dependence of the kinetics.

4. Use 2DES data to test different excitonic models of the BRC. 2DES data has recently been used to refine excitonic models of the Photosystem II reaction center $[69,79,80]$ based on the Novoderezkin model $[81,82]$. 2D data from BRCs can also potentially be used to test proposed models of BRC charge separation processes [83-86].

This thesis is organized into three main chapters. Chapter 2 focuses on the experimental implementation of 2DES, beginning with a brief introduction of the technique, followed by details specific to our $2 \mathrm{D}$ spectrometer. A significant portion of the project involved developing a degenerate optical parametric amplifier to generate laser pulses spanning the entire 700-900nm BRC absorption region, which is described at the end of the chapter.

Chapter 3 discusses data analysis and global fitting methods, and the extension of transient absorption fitting techniques to multi-dimensional data sets. It explores the commonly used 2DES global fitting approaches, and their limitations for uniquely identifying sequential and parallel reaction kinetics. An improved 2D kinetic fitting approach is presented which greatly reduces the number of free parameters by using a common set of basis spectra for all excitation frequencies, and models the excitation-dependence based on linear absorption data. The possible extension of the fitting technique to multiple data sets of different 
types is discussed as a means of further constraining the fitting results. The last section discusses the polarization-dependence of 2D spectra, its implications for data fitting, and the decomposition of 2D spectra into orthogonal polarization components.

Chapter 4 presents 2DES measurements of two BRC mutants from $R$. capsulatus. The results from the conventional global analysis techniques described in Chapter 3 are discussed, as well as a close comparison of our data with published transient absorption studies. The improved 2D excitation-dependent global fitting approach is used to test the 2DES data against different kinetic models. Chapter 5 summarizes the results, and discusses future directions for the project. 


\section{CHAPTER 2}

\section{TWO-DIMENSIONAL ELECTRONIC SPECTROSCOPY}

Studying energy and charge transfer dynamics in photosynthetic light harvesting systems is particularly challenging due to the spectral overlap between pigments and the subpicosecond timescales involved [3, 4]. Transient absorption (TA) spectroscopy has proven to be a powerful tool for studying ultrafast molecular dynamics in a wide variety of systems, but spectral congestion in RCs, combined with complicated, multiply-branched energy transfer pathways, makes interpretation of TA spectra difficult, especially when only one or a few excitation frequencies are measured.

Two-dimensional electronic spectroscopy (2DES) measures the optical response with respect to excitation and detection frequencies, offering a number of advantages over linear and TA spectroscopy. The excitation frequency dependence of BRC dynamics is particularly informative since the $\mathrm{P}, \mathrm{B}$ and $\mathrm{H}$ absorption bands are sufficiently seperated to selectively excite different subsets of pigments. TA spectroscopy is fundamentally limited by the timebandwidth product of the excitation pulse, resulting in a trade-off between time resolution and excitation frequency resolution [87]. Given that energy transfer has been observed on timescales < 100fs in BRCs $[16,54,58]$, this limitation actually becomes signficant, requiring pump bandwidths of tens of nanometers to observe the early energy transfer dynamics. 2DES overcomes this limitation, acquiring broadband excitation-dependent spectra with high resolution in both excitation frequency and time. 


\subsection{DES Theory}

2DES measures the optical response of a system induced by interactions with a sequence of laser pulses, so the signal of interest necessarily has a nonlinear dependence on the electric field applied by the pulse sequence. The nonlinear response of a bulk sample can be described as an expansion of the polarization density $\boldsymbol{P}(t)$ with respect to the electric field [88]:

$$
\begin{aligned}
\boldsymbol{P}(t) & =\epsilon_{0}\left(\chi^{(1)} \boldsymbol{E}(t)+\chi^{(2)} \boldsymbol{E}^{2}(t)+\chi^{(3)} \boldsymbol{E}^{3}(t)+\ldots\right) \\
& =\boldsymbol{P}^{(1)}(t)+\boldsymbol{P}^{(2)}(t)+\boldsymbol{P}^{(3)}(t)+\ldots
\end{aligned}
$$

The first-order susceptibility $\chi^{(1)}$ gives rise to the linear dispersion and absorption effects of classical optics. Separating $\boldsymbol{P}(t)$ into the linear term $\boldsymbol{P}^{(1)}(t)$ and nonlinear terms $\boldsymbol{P}^{(N L)}(t)$, a nonlinear wave equation for the electic field follows from Maxwell's equations:

$$
\nabla^{2} \boldsymbol{E}-\frac{n^{2}}{c^{2}} \frac{\partial^{2}}{\partial t^{2}} \boldsymbol{E}=\frac{1}{\epsilon_{0} c^{2}} \frac{\partial^{2}}{\partial t^{2}} \boldsymbol{P}^{N L}
$$

The homogeneous solutions to Equation 2.3 are classical electromagnetic waves, and $\boldsymbol{P}^{N L}(t)$ is effectively a forcing term, which itself depends on the local electric field. Crossterms between electric field components in $\boldsymbol{P}^{N L}(t)$ result in nonlinear mixing processes. The second-order susceptibility $\chi^{(2)}$ is responsible for the second-harmonic generation and difference-frequency mixing processes used in our optical parametric amplifiers, as well as sum-frequency mixing and optical rectification. $\chi^{(3)}$ processes include third harmonic generation, cross-polarized wave generation, the optical Kerr effect, and four-wave mixing.

2DES and transient absorption spectroscopy both measure signals that are third-order in the electric field. The reaction kinetics information we wish to probe is encoded in the time-dependent third-order susceptibility $\chi^{(3)}$, a rank-4 tensor relating the polarization components of each of the three interacting fields to the generated response. In linear optics, the polarization induced by the electric field is the convolution of the field with a response function $\boldsymbol{R}^{(1)}(t)$ characterizing the system:

$$
\boldsymbol{P}^{(1)}(t)=\int_{-\infty}^{\infty} d \tau \boldsymbol{R}^{(1)}(t ; \tau) \cdot \boldsymbol{E}(\tau)
$$


where in the general case $\boldsymbol{R}^{(1)}$ is a second-order tensor, since each component of $\boldsymbol{P}^{(1)}$ can depend on the three polarization components of $\boldsymbol{E}$. The third-order polarization can analogously be described as a convolution of the third-order response $\boldsymbol{R}^{(3)}\left(t, \tau_{1}, \tau_{2}, \tau_{3}\right)$ :

$$
\boldsymbol{P}^{(3)}(t)=\int_{-\infty}^{\infty} d \tau_{1} \int_{-\infty}^{\infty} d \tau_{2} \int_{-\infty}^{\infty} d \tau_{3} \boldsymbol{R}^{(3)}\left(t ; \tau_{1}, \tau_{2}, \tau_{3}\right) \boldsymbol{E}\left(\tau_{1}\right) \boldsymbol{E}\left(\tau_{2}\right) \boldsymbol{E}\left(\tau_{3}\right)
$$

where $\boldsymbol{R}^{(3)}$ is a rank 4 tensor. The goal of $2 \mathrm{DES}$ is to extract the response function $\boldsymbol{R}^{(3)}$ by measuring the nonlinear signal $\boldsymbol{E}_{\text {sig }}(t)$ generated from $\partial^{2} \boldsymbol{P}^{(3)}(t) / \partial t^{2}$. This is done by applying an electric field that is a sequence of pulses $E(t)=E_{1}\left(t-t_{1}\right)+E_{2}\left(t-t_{2}\right)+E_{3}(t-$ $\left.t_{3}\right)$. In the limit where $E_{i}(t)=\delta\left(t-t_{i}\right)$, then $P^{(3)}(t)=R^{(3)}\left(t ; t_{1}, t_{2}, t_{3}\right)$, and the response function can be reconstructed by scanning the pulse time delays. The frequency response (or susceptibility) of the system, $\boldsymbol{\chi}^{(3)}\left(\omega_{1}, \omega_{2}, \omega_{3}\right)$ can then be obtained by Fourier transformation with respect to $t, t_{1}, t_{2}$, and $t_{3}$.

For a three-pulse experiment, the electric field in Equation 2.5 is the sum of the fields from all three pulses, each with arbitrary polarization $\hat{e}_{j}$, carrier frequency $\omega_{j}$, wavevector $\vec{k}_{j}$, constant phase factor $\phi_{j}$, and envelope function $E_{j}(t)$ :

$$
\begin{aligned}
\boldsymbol{E}_{\boldsymbol{j}}(t, \vec{r}) & =E_{j}(t) e^{i\left(\vec{k}_{j} \cdot \vec{r}-\omega_{j} t+\phi_{j}\right)} \hat{e}_{j}+c . c . \\
\boldsymbol{E}(t, \vec{r}) & =\boldsymbol{E}_{1}+\boldsymbol{E}_{2}+\boldsymbol{E}_{3}
\end{aligned}
$$

Inserting this expression into Equation 2.5 and expanding, there are $6^{3}=216$ terms (including conjugates). The 216 terms in $\boldsymbol{P}^{(3)}(t, \vec{r})$ will have frequencies $\omega= \pm \omega_{l} \pm \omega_{m} \pm \omega_{n}$, and spatially dependent phase factors $e^{i\left( \pm \vec{k}_{l} \pm \vec{k}_{m} \pm \vec{k}_{n}\right) \cdot \vec{r}}$, for $\{l, m, n\}=\{1,2,3\}$. In practice, however, only a few of these terms are measured in a 2DES experiment. Specific signal contributions can be isolated by controlling the directions, polarizations, relative phases, and time-ordering of the input pulses, and selecting the detected direction and carrier frequency of the emitted signals [89].

The pulse sequence used for the 2DES experiment is shown in Figure 2.1. $E_{1}(t)$ and $E_{2}(t)$ serve as the pump pulses, and $E_{3}(t)$ is the probe pulse. The polarization $\boldsymbol{P}^{(3)}(t)$ oscillates in response to the pulse sequence according to Equation 2.5 and radiates a signal 
field according to Equation 2.3, with direction $\vec{k}_{s}= \pm \vec{k}_{1} \pm \vec{k}_{2} \pm \vec{k}_{3}$ and corresponding carrier frequency $\omega_{s}=\omega_{1} \pm \omega_{2} \pm \omega_{3}$. The signal field is then mixed with a reference field (local oscillator) and measured in a spectrometer by heterodyne detection, giving the detection frequency dependence $\omega_{3}$. Fourier transformation with respect to the coherence time $\tau$ yields the excitation axis $\omega_{1}$.

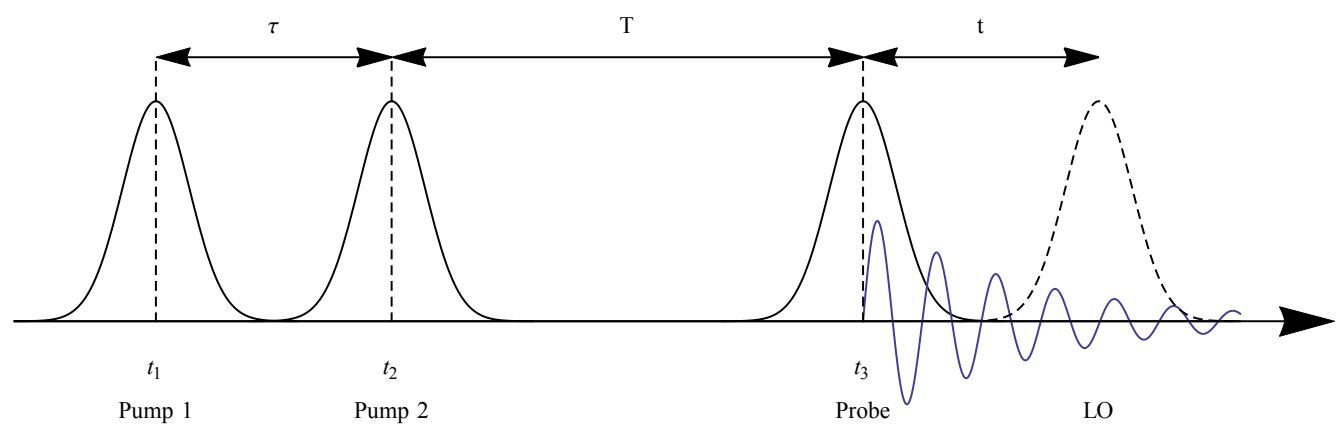

Figure 2.1: Pulse sequence used for 2DES experiments. $\tau$ : coherence time, T: population time, t: probe/local oscillator delay (fixed)

If we restrict our attention to signals emitted at the probe frequency $\omega_{3}$, with the carrier frequencies $\omega_{1}$ and $\omega_{2}$ of the pumps being equal, we get the two terms: $\omega_{s}=-\omega_{1}+\omega_{2}+\omega_{3}=$ $\omega_{3}$ and $\omega_{s}=+\omega_{1}-\omega_{2}+\omega_{3}=\omega_{3}$. The signals associated with these two terms in the $\boldsymbol{P}^{(3)}(t)$ expansion add constructively in the phase-matched directions $\vec{k}_{\mathrm{R}}=-\vec{k}_{1}+\vec{k}_{2}+\vec{k}_{3}$ and $\vec{k}_{\mathrm{NR}}=\vec{k}_{1}-\vec{k}_{2}+\vec{k}_{3}$, respectively, and are termed the rephasing and nonrephasing signals [90]. Figure 2.2 illustrates the phase-matched signal directions for the "box-CARS" beam geometry in which the wavevectors of the incident pump and probe beams form three corners of a square. The $\vec{k}_{1}$ direction corresponds to the first pump pulse in the pulse sequence from Figure 2.1. Switching the pulse order of the two pumps exchanges the directions of $\vec{k}_{\mathrm{R}}$ and $\vec{k}_{\mathrm{NR}}$.

The macroscopic polarization $\boldsymbol{P}(t)$ from Equation 2.1 arises from the sum of the field/matter interactions of each sample molecule, which can be represented in terms of the expectation value of the dipole operator $[89,90]$ :

$$
\boldsymbol{P}(t)=N \operatorname{Tr}[\rho(t) \mu]
$$




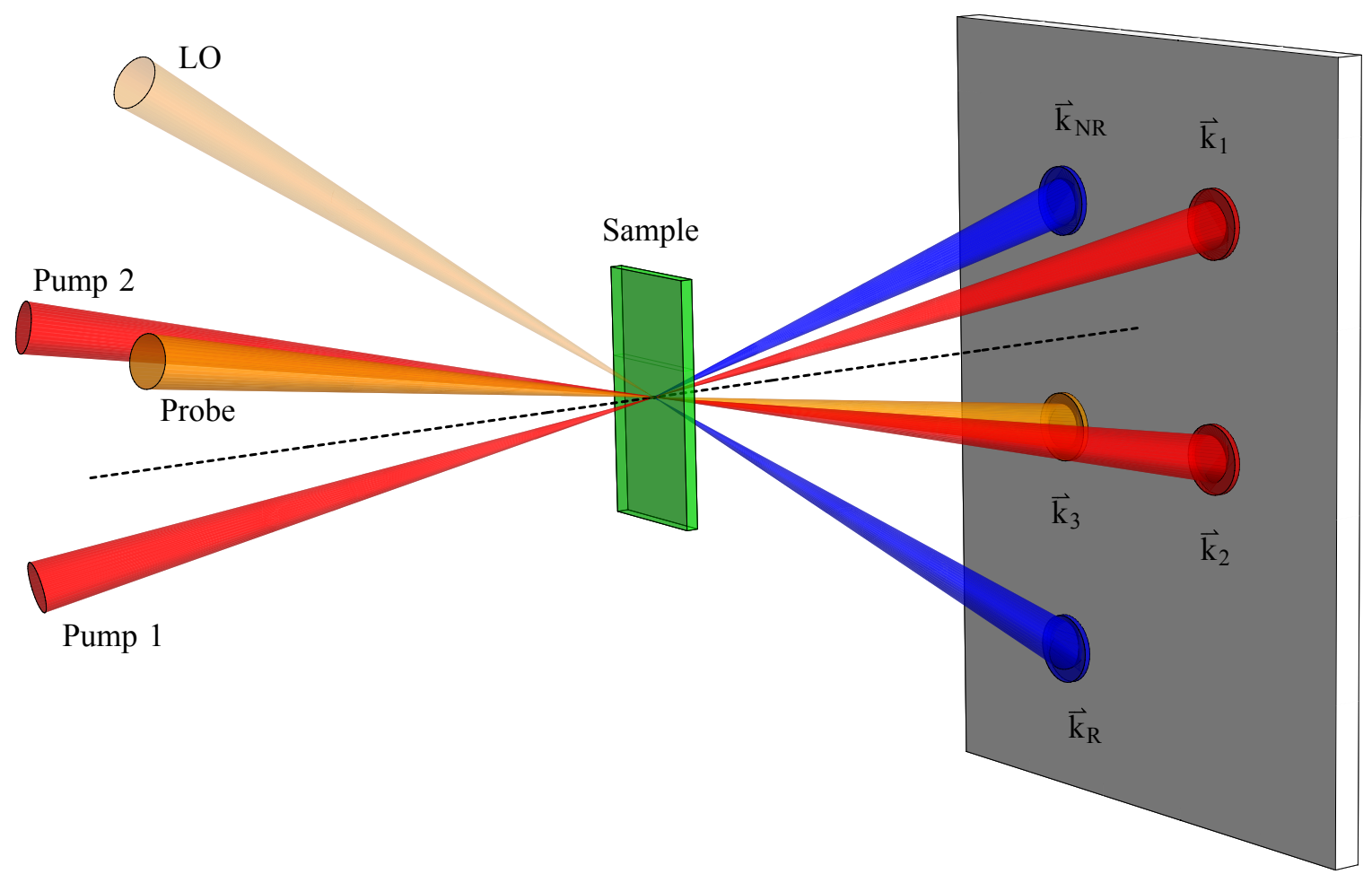

Figure 2.2: Phase-matched four wave mixing signals in the box-CARS geometry. $\vec{k}_{\mathrm{R}}$ and $\vec{k}_{\mathrm{NR}}$ correspond to rephasing and nonrephasing signals, respectively.

$\rho(t)$ is the density matrix, $\mu$ is the dipole operator, and $N$ is the number density of sample molecules. Under the assumption that the coupling between the external field and the system is weak, the time-dependent density matrix can be expanded perturbatively in powers of the field, similarly to $\boldsymbol{P}^{(N L)}(t)$ :

$$
\rho(t)=\rho^{(0)}(t)+\rho^{(1)}(t)+\rho^{(2)}(t)+\ldots
$$

where the $\mathrm{n}^{\text {th }}$-order density matrix term involves $n$ field/matter interactions. The nonlinear polarization measured by $2 \mathrm{DES}$ is then related to the time evolution of the density matrix through the nonlinear response function $S^{(3)}$ [89]:

$$
\begin{gathered}
P^{(3)}(t)=\int_{0}^{\infty} d t_{3} \int_{0}^{\infty} d t_{2} \int_{0}^{\infty} d t_{1} S^{(3)}\left(t_{3}, t_{2}, t_{1}\right) \\
E\left(t-t_{3}\right) E\left(t-t_{3}-t_{2}\right) E\left(t-t_{3}-t_{2}-t_{1}\right) \\
S^{(3)}\left(t_{3}, t_{2}, t_{1}\right)=\left(\frac{i}{\hbar}\right)^{3}\left\langle\left\langle\mu \mid G\left(t_{3}\right) V G\left(t_{2}\right) V G\left(t_{1}\right) V \rho(-\infty)\right\rangle\right\rangle
\end{gathered}
$$


Equation 2.10 is in the form of Equation 2.5, but in terms of the time intevals between successive field interactions. In Equation 2.11, $\rho$ is the density operator in Louiville space, $L$ is a Louiville operator representing a field/matter interaction through dipole coupling, and $G(t)$ is the Louiville space Green function which propagates the system between interactions.

The physical significance of the rephasing and nonrephasing signal components can be better understood in terms of the time evolution of different terms of the density matrix. In terms of the pulse sequence defined in Figure 2.1, the first pulse puts the system in a coherent state (i.e. an off-diagonal density matrix element), the second pulse puts the system in a ground or excited state population, and the third pulse induces another coherent state which oscillates at the detection frequency. The rephasing term is measured in stimulated photon echo experiments, and corresponds to the system evolving with conjugate frequencies during the coherence time $\tau$ and detection time $t[90]$. The dephasing between different molecules during the coherence time evolves with opposite sign during the detection time, so that the phases realign at $t=\tau$ [91]. The nonrephasing term corresponds to density matrix evolution of the same sign during coherence and detection times, and therefore does not produce an echo signal. The absorptive component of the optical response is the sum of the rephasing and nonrephasing terms [90, 92, 93]. Absorptive 2D spectra are more useful than the rephasing spectra for analyzing population kinetics, as they are directly analogous to transient absorption measurements, and probe the absorption difference spectra associated with populations of each excited state. All $2 \mathrm{D}$ spectra shown in this thesis are the real absorptive spectra obtained from the rephasing and nonrephasing signals.

The absorptive 2D spectrum can distinguish between homogeneous and inhomogeneous broadening, and reveals information on the coupling and energy transfer between different states. Figure 2.3 illustrates an inhomogeneously broadened absorption band comprised of a distribution of narrow lineshapes. In the corresponding $2 \mathrm{D}$ spectrum, the signal appears along the $\omega_{e x}=\omega_{\text {det }}$ diagonal. The excitation and detection frequencies are correlated, with the inhomogeneous and homogeneous linewidths corresponding to the diagonal and 
anti-diagonal widths of the 2D spectrum, respectively.
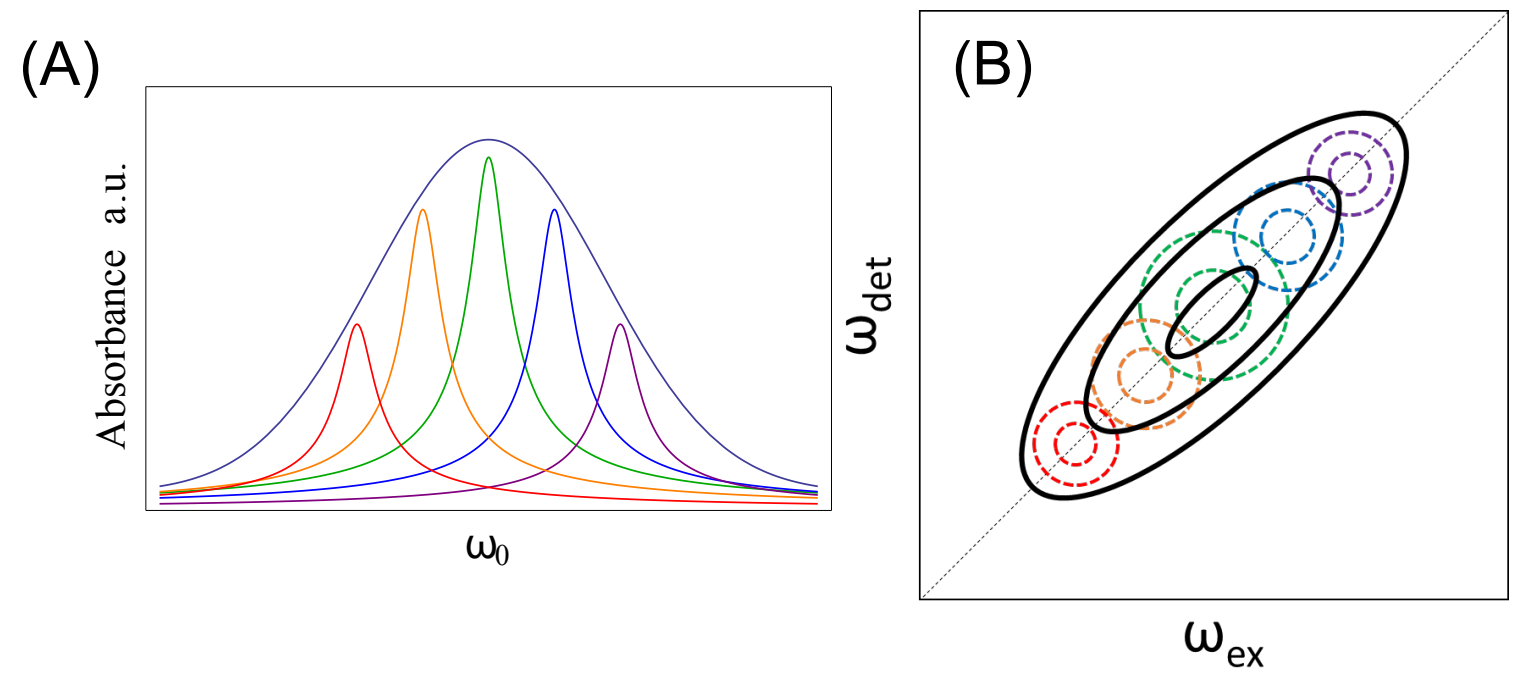

Figure 2.3: Illustration of the 2D spectrum of an inhomogeneously broadened lineshape. (A) Linear absorption spectrum comprised of a Gaussian distribution of narrow Lorentzian peaks. (B) Representative sketch of the associated absorptive 2D spectrum.

Figure 2.4 illustrates the types of features appearing in 2D spectra in simple 2- and 3-level systems. Figure 2.4A represents two uncoupled systems, one of which has a second excited state. Positive peaks appear at $\omega_{1}$ and $\omega_{2}$ corresponding to stimulated emission and ground state bleaching of each system. An additional negative excited state absorption peak appears at $\omega_{\text {det }}=\omega_{2^{\prime}}$, which represents an increased absorption at frequency $\omega_{2^{\prime}}$ upon excitation at frequency $\omega_{2}$. Figure 2.4B shows two weakly coupled two-level systems exhibiting energy transfer from system 2 to system 1 . At $\mathrm{T}=0$, we see the inhomogeneously broadened diagonal peaks from the two systems. As time evolves, a cross-peak appears below the diagonal, indicating that excitation at $\omega_{2}$ populated state 1 , which emitted a signal at $\omega_{1}$. In terms of the BRC, this case describes the weak coupling between the monomeric Bchl and BPheo, where the excitations are mostly localized on the individual chromophores and population transfer is mostly unidirectional from the higher energy BPheo to the lower energy Bchl state. Figure $2.4 \mathrm{C}$ shows the case of two strongly coupled chromophores. The energy eigenstates in this case are delocalized, resulting in two excitonically split levels, and cross-peaks appearing at early population times. 
(A)

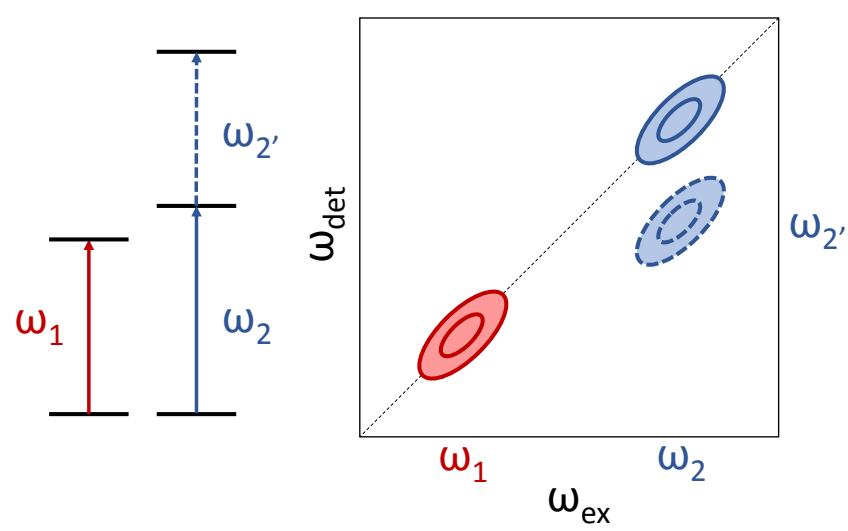

(B)
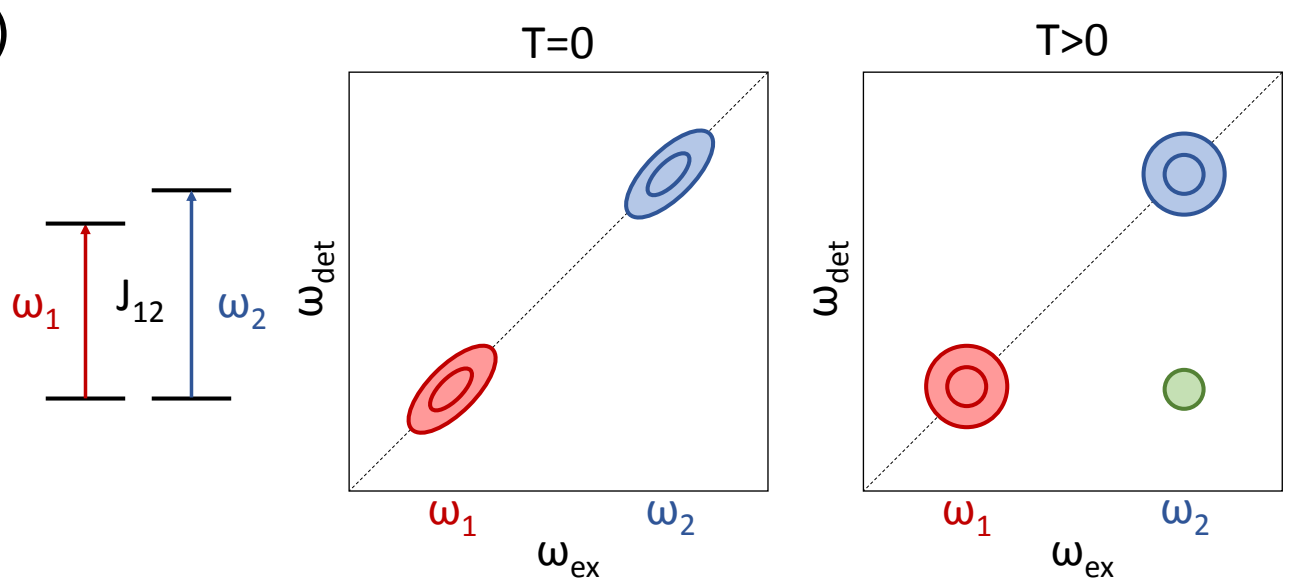

(C)
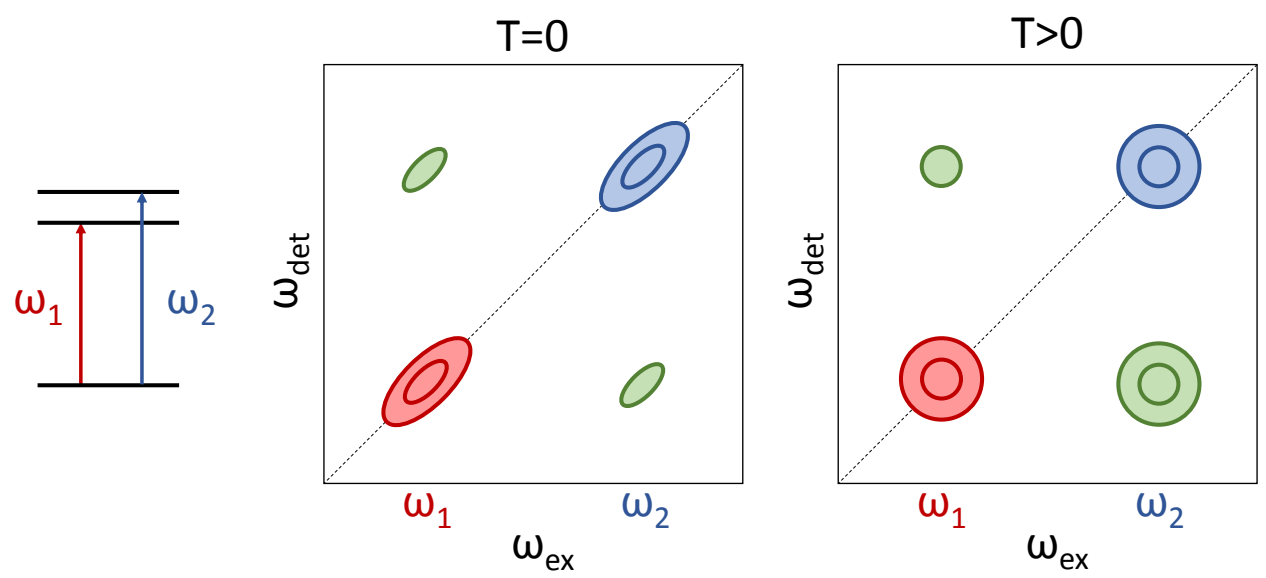

Figure 2.4: Features appearing in absorptive 2D spectra for several simple systems. (A) Two uncoupled systems, with excited state absorption. (B) Two weakly coupled two-level systems with downhill energy transfer. (C) Two strongly coupled two-level systems exhibiting excitonic splitting. Figure adapted from [94]. 


\subsection{Experimental Implementation}

A schematic of the 2DES setup is shown in Figure 2.5 [94]. A Ti:sapphire regenerative amplifier (Spectra Physics Spitfire Pro) produces $4 \mathrm{~mJ}, 50 \mathrm{fs}$ pulses at a $500 \mathrm{~Hz}$ repetition rate. A portion of the output feeds a home-built Degenerate Optical Parametric Amplifier (DOPA) which outputs $8 \mu \mathrm{J}$ pulses with $680-920 \mathrm{~nm}$ bandwidth. The DOPA design is discussed in more detail in Section 2.3. The DOPA output is then split, with $80 \%$ power allocated for the pump and $20 \%$ for the probe. The pump beam is pre-compressed with a pair of broadband chirped mirrors before entering an acousto-optic pulse shaper (Dazzler, Fastlite). The Dazzler generates a duplicate pump pulse with a phase-stable time delay $\tau$ (used for the coherence time scanning) and arbitrary phases $\phi_{1}$ and $\phi_{2}$ for the first and second pump pulses. It can also be used for fine adjustments to pulse compression, amplitude shaping, and spectral shaping for narrow-bandwidth experiments. The probe beam passes through a different set of broadband chirped mirrors, and is further compressed using a spatial light modulator (FemtoJock, Biophotonics Solutions). The relative delay between the pump and probe paths is adjusted with a retroreflector on a linear stage, which scans the population time $T$.

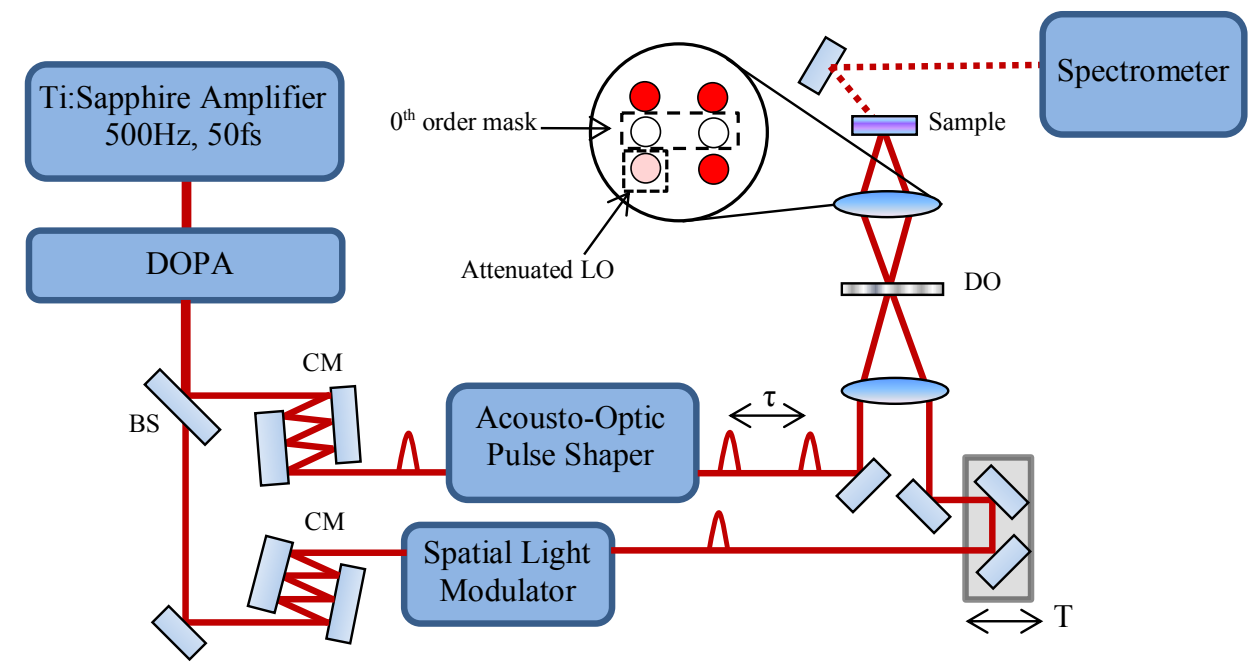

Figure 2.5: Layout of 2DES setup based on the design of Fuller et al. [94]. DOPA: degenerate optical parametric amplifier, BS: beam splitter, CM: chirped mirror, DO: diffractive optic, LO: local oscillator 
The pump and probe beams are then focused onto a diffractive optic $(20 \mathrm{~g} / \mathrm{mm})$ to a spot size of approximately $200 \mu \mathrm{m}$, producing only odd-order diffracted beams. A spatial filter is used to block the unused diffracted beams, as shown in Figure 2.6, selecting the \pm 1 -order pump beams and the +1 -order probe beam. The +3 -order probe beam is used for the local oscillator, and a fused silica window is inserted into the local oscillator path to impart the signal/LO delay of $800 \mathrm{fs}$ for heterodyne detection. A spherical mirror then images the diffractive optic to the sample plane, ensuring that all four beams come to a focus and cross at the same point at the sample, in a "box-CARS" geometry. The reflective coating of the imaging mirror is etched to attenuate LO reflection. By using the etched mirror and selecting the lower-amplitude +3 -order beam, the LO intensity is sufficiently reduced to avoid saturation of the spectrometer CCD.

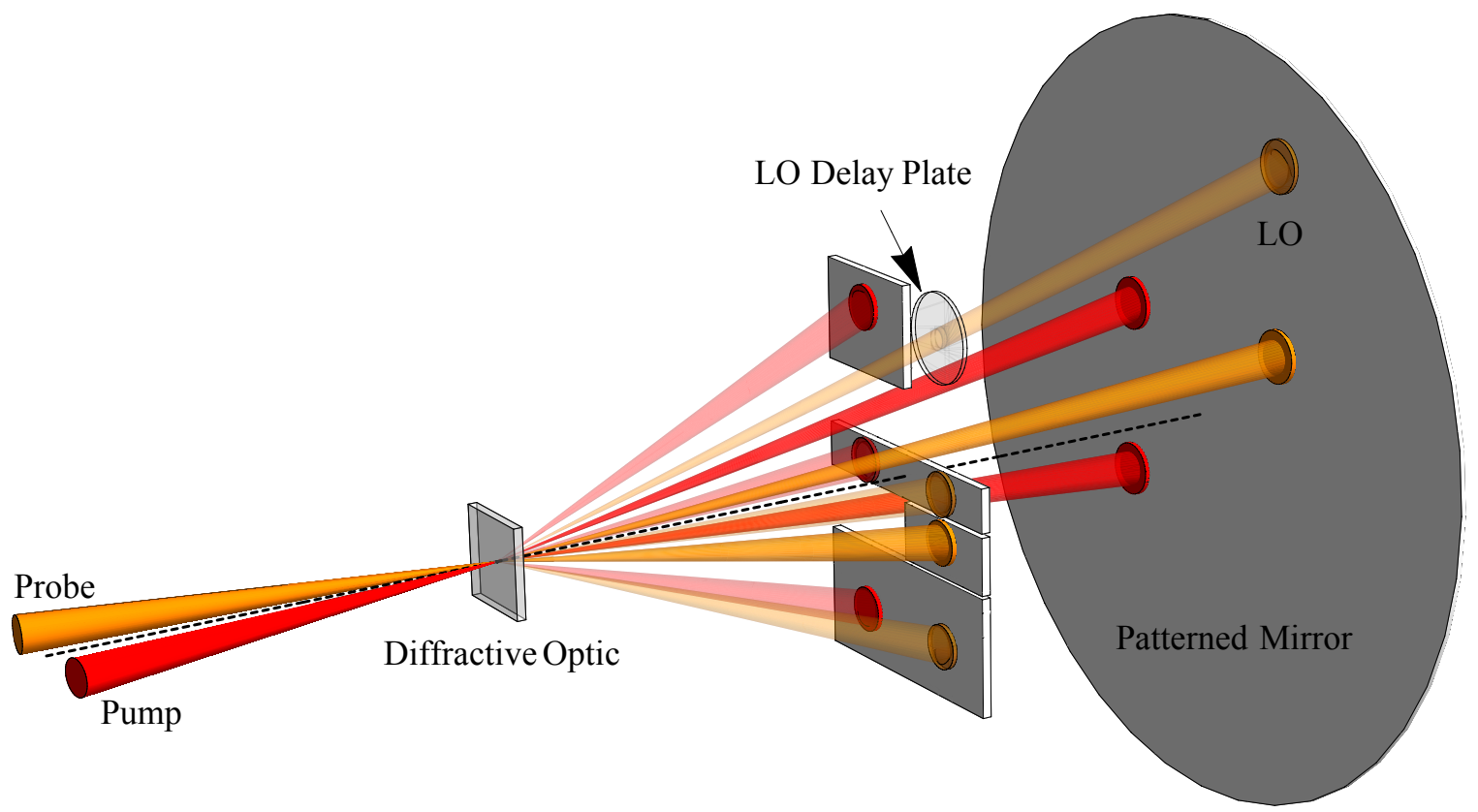

Figure 2.6: Diffractive optic beam geometry used to make box-CARS configuration. Beam blocks are placed in front of the imaging mirror to select the pump, probe and LO beams. A fused silica window is inserted in the LO path to impart a time delay for spectral interferometry. 


\subsubsection{Phase Cycling}

In this configuration, each of the two diffracted pump beams contains two time-delayed pump pulses. There are four pairs of pump pulse interactions which generate signals in the LO direction $\vec{k}_{s}=-\vec{k}_{1}+\vec{k}_{2}+\vec{k}_{3}$. Figure 2.7 illustrates the four combinations and their dependence on the relative phase $\Delta \phi$ between the pump pulses at different time delays. The detected signal is a linear combination of the rephasing signal, nonrephasing signal, and two transient grating signals at different delays [94].
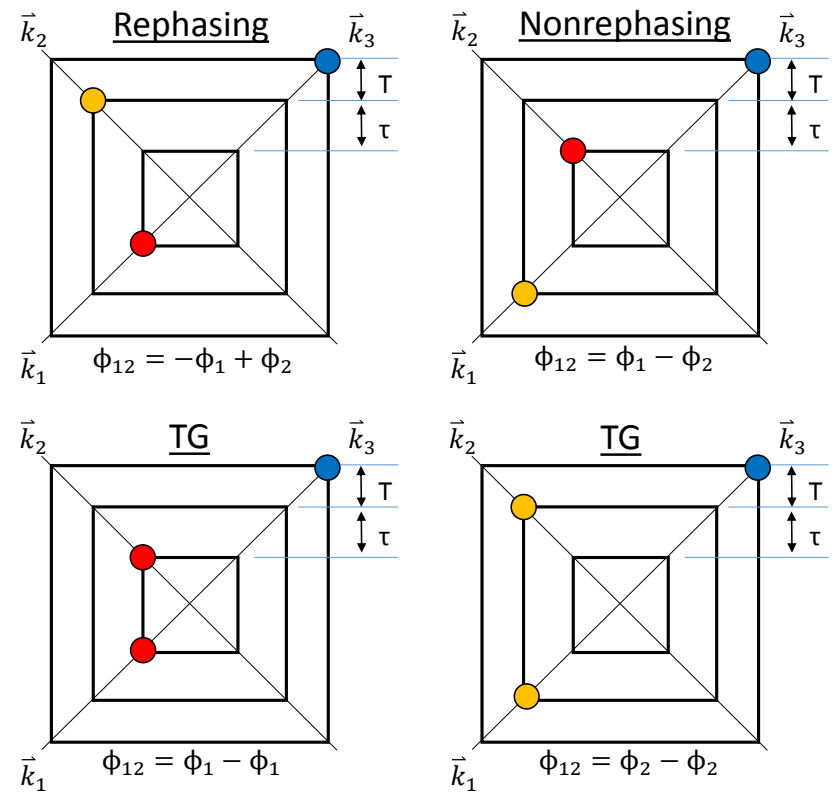

Figure 2.7: Pulse timing diagrams for the four pump-pair interactions generating signal in the $-\vec{k}_{1}+\vec{k}_{2}+\vec{k}_{3}$ direction. Pulses closer to the center arrive at the sample first. Red and orange circles represent pump pulses with phases $\phi_{1}$ and $\phi_{2}$ respectively, and blue represents the probe pulse. Figure adapted from [95].

Define the four pump pulses as $E_{i j}$, where $i$ is the time ordering with $i=1$ arriving first, and $j$ labels the associated wavevector $\vec{k}_{1}$ or $\vec{k}_{2}$. Then the four signal terms in the $-\vec{k}_{1}+\vec{k}_{2}+\vec{k}_{3}$ direction have the following phase dependence: 


$$
\begin{aligned}
\boldsymbol{S}_{\mathrm{R}} & \propto \boldsymbol{E}_{11}^{*} \boldsymbol{E}_{22} \boldsymbol{E}_{3}=E_{1} E_{2} E_{3} \exp \left[i\left(-\vec{k}_{1}+\vec{k}_{2}+\vec{k}_{3}\right) \cdot \vec{r}+i\left(\phi_{2}-\phi_{1}+\phi_{3}\right)-i \omega_{s} t\right] \\
\boldsymbol{S}_{\mathrm{NR}} & \propto \boldsymbol{E}_{12} \boldsymbol{E}_{21}^{*} \boldsymbol{E}_{3}=E_{1} E_{2} E_{3} \exp \left[i\left(-\vec{k}_{1}+\vec{k}_{2}+\vec{k}_{3}\right) \cdot \vec{r}+i\left(\phi_{1}-\phi_{2}+\phi_{3}\right)-i \omega_{s} t\right] \\
\boldsymbol{S}_{\mathrm{TG} 1} & \propto \boldsymbol{E}_{11}^{*} \boldsymbol{E}_{12} \boldsymbol{E}_{3}=E_{1} E_{2} E_{3} \exp \left[i\left(-\vec{k}_{1}+\vec{k}_{2}+\vec{k}_{3}\right) \cdot \vec{r}+i\left(\phi_{1}-\phi_{1}+\phi_{3}\right)-i \omega_{s} t\right] \\
\boldsymbol{S}_{\mathrm{TG} 2} & \propto \boldsymbol{E}_{21}^{*} \boldsymbol{E}_{22} \boldsymbol{E}_{3}=E_{1} E_{2} E_{3} \exp \left[i\left(-\vec{k}_{1}+\vec{k}_{2}+\vec{k}_{3}\right) \cdot \vec{r}+i\left(\phi_{2}-\phi_{2}+\phi_{3}\right)-i \omega_{s} t\right]
\end{aligned}
$$

The rephasing and nonrephasing signals have conjugate phase dependence on $\Delta \phi=\phi_{1}-\phi_{2}$, while the two TG signals have no $\Delta \phi$-dependence. Making three measurements $S_{1}, S_{2}$, and $S_{3}$ with relative pump phases $\Delta \phi \in\left\{\Delta \phi_{1}, \Delta \phi_{2}, \Delta \phi_{3}\right\}$ is sufficient to isolate $S_{\mathrm{R}}, S_{\mathrm{NR}}$ and $S_{\mathrm{TG}}$ [94]:

$$
\left[\begin{array}{l}
S_{1} \\
S_{2} \\
S_{3}
\end{array}\right]=\left[\begin{array}{lll}
e^{i \Delta \phi_{1}} & e^{-i \Delta \phi_{1}} & 1 \\
e^{i \Delta \phi_{2}} & e^{-i \Delta \phi_{2}} & 1 \\
e^{i \Delta \phi_{3}} & e^{-i \Delta \phi_{3}} & 1
\end{array}\right]\left[\begin{array}{c}
S_{\mathrm{R}} \\
S_{\mathrm{NR}} \\
S_{\mathrm{TG}}
\end{array}\right]
$$

In addition to separating the phase-matched signal components, pump phase cycling can also be used to distinguish the four-wave mixing signal of interest from unwanted scatter terms which propagate in the signal direction. By taking a pair of measurements $S_{0}$ and $S_{\pi}$ with pump phases $\phi_{1}=\phi_{2}=0$ and $\phi_{1}=\phi_{2}=0$, respectively, we find that the rephasing and nonrephasing signals are unaffected by the change of phase, while scatter terms involving interactions with only one pump are conjugated [94-96]. Adding the two gives:

$$
\begin{aligned}
\boldsymbol{S}_{0}+\boldsymbol{S}_{\pi} & \propto\left(\boldsymbol{S}_{\mathrm{R}}(0)+\boldsymbol{S}_{\mathrm{NR}}(0)+\boldsymbol{S}_{\mathrm{PS}}(0)\right)+\left(\boldsymbol{S}_{\mathrm{R}}(\pi)+\boldsymbol{S}_{\mathrm{NR}}(\pi)+\boldsymbol{S}_{\mathrm{PS}}(\pi)\right) \\
& =\left(\boldsymbol{S}_{\mathrm{R}}+\boldsymbol{S}_{\mathrm{NR}}\right) e^{i(0-0)}+\left(\boldsymbol{S}_{\mathrm{R}}+\boldsymbol{S}_{\mathrm{NR}}\right) e^{i(\pi-\pi)}+\boldsymbol{S}_{\mathrm{PS}}\left(e^{i(0)}+e^{i(\pi)}\right) \\
& =2\left(\boldsymbol{S}_{\mathrm{R}}+\boldsymbol{S}_{\mathrm{NR}}\right)
\end{aligned}
$$

where $\boldsymbol{S}_{\mathrm{PS}}$ are scatter terms involving any of the four pump pulses. Note that this will not eliminate scatter from the probe, since $\boldsymbol{E}_{\mathrm{Pr}} \boldsymbol{E}_{\mathrm{LO}}^{*}$ has no pump phase dependence. However, using the three phase-cycle scheme from Equation 2.12, probe scatter terms have the same $\Delta \phi$-dependence as the two $\boldsymbol{S}_{\mathrm{TG}}$ signals. By acquiring $S_{0}$ and $S_{\pi}$ measurements for each of the three $\Delta \phi$ values, we get a 6 phase-cycle scheme which isolates the $\boldsymbol{S}_{\mathrm{R}}$ and $\boldsymbol{S}_{\mathrm{NR}}$ signals 
while suppressing scattered light from the pump and probe. Using the following six phases:

$$
\left\{\phi_{1}, \phi_{2}\right\} \in\{\{0,0\},\{0,2 \pi / 3\},\{0,4 \pi / 3\},\{\pi, \pi\},\{\pi, 5 \pi / 3\},\{\pi, 7 \pi / 3\}\}
$$

with associated signals $\left\{\boldsymbol{S}_{6}, \ldots, \boldsymbol{S}_{6}\right\}$, the pump scatter terms can be removed by adding pairs of signals $\left\{\boldsymbol{S}_{1}^{\prime}, \boldsymbol{S}_{2}^{\prime}, \boldsymbol{S}_{3}^{\prime}\right\}=\left\{\boldsymbol{S}_{1}+\boldsymbol{S}_{4}, \boldsymbol{S}_{2}+\boldsymbol{S}_{5}, \boldsymbol{S}_{3}+\boldsymbol{S}_{6}\right\}$, which are then used in Equation 2.12 to get the rephasing and nonrephasing signals [94]. This operation can be expressed in matrix form as:

$$
\left[\begin{array}{c}
\boldsymbol{S}_{\mathrm{R}} \\
\boldsymbol{S}_{\mathrm{NR}} \\
\boldsymbol{S}_{\mathrm{TG}}+\boldsymbol{S}_{\mathrm{Pr}}
\end{array}\right]=\left[\begin{array}{lll}
e^{i \Delta \phi_{1}} & e^{-i \Delta \phi_{1}} & 1 \\
e^{i \Delta \phi_{2}} & e^{-i \Delta \phi_{2}} & 1 \\
e^{i \Delta \phi_{3}} & e^{-i \Delta \phi_{3}} & 1
\end{array}\right]^{-1}\left[\begin{array}{cccccc}
1 & 0 & 0 & 1 & 0 & 0 \\
0 & 1 & 0 & 0 & 1 & 0 \\
0 & 0 & 1 & 0 & 0 & 1
\end{array}\right]\left[\begin{array}{c}
\boldsymbol{S}_{1} \\
\boldsymbol{S}_{2} \\
\boldsymbol{S}_{3} \\
\boldsymbol{S}_{4} \\
\boldsymbol{S}_{5} \\
\boldsymbol{S}_{6}
\end{array}\right]
$$

Similar scatter subtraction can be achieved in other 2D setups using either shutters or optical choppers to measure signals from different combinations of beams, and isolating the terms depending on all three. The advantage of the phase-cycling approach described here is that the signals of interest are contained in each laser shot, so the scatter subtraction is achieved without any reduction in duty cycle. Another advantage of the four-pump configuration is that the rephasing and nonrephasing signals are acquired simultaneously for each laser shot, so it is not neccessary to scan negative values of the coherence time. Not only does this reduce the acquisition time, but it also reduces the effect of long-term laser amplitude fluctuations on the reconstructed absorptive 2D spectrum. By performing the phase-cycling with an acousto-optic pulse shaper, the phases can be cycled with each laser shot, which reduces scatter subtraction errors resulting from laser fluctuations.

Control of the pump phases $\phi_{1}$ and $\phi_{2}$ with the Dazzler also enables measurement of the coherence time axis in a rotating reference frame. After excitation by the first pump pulse, the system evolves with respect to the coherence time with a frequency equal to the induced transition. For an 800nm pump pulse, this translates to a period of $2.7 \mathrm{fs}$, requiring a $\tau$ sample spacing of $<1.3 \mathrm{fs}$. In $\mathrm{BRC}$ experiments at $77 \mathrm{~K}$, the coherence decay time (i.e. dephasing time) is on the order of 100fs. Supposing $1 \mathrm{fs}$ spacing and scanning to $400 \mathrm{fs}$, and 
applying six phase cycles for each time, this requires 2400 waveforms to acquire a single $2 \mathrm{D}$ spectrum. By applying an additional $\tau$-dependent phase factor $\phi_{1}(\tau)=\omega_{L} \tau$, the coherence time points are sampled in a reference frame rotating at the specified phase-lock frequency $\omega_{L}[94,95,97]$. This phase factor combines the with spectral phase $e^{-i \omega \tau}$ applied to the first pump pulse to shift the observed coherence frequency from $\omega_{0}$ to $\omega_{0}-\omega_{L}$. After Fourier transforming with respect to $\tau$ sampled in the rotating frame, the DC point in the Fourier transform is shifted to $\omega_{L}$. By setting the lock frequency to the central frequency of the pump, we detect the slowly varying decay envelope, which only requires $\tau$ sampling of $10 \mathrm{fs}$. In a typical experiment, $\tau$ is scanned from 0 to $390 \mathrm{fs}$ with $10 \mathrm{fs}$ spacing and six phase cycles, for a total of 240 waveforms. At a $500 \mathrm{~Hz}$ repetition rate, this gives $1252 \mathrm{D}$ spectra per minute. By scanning the coherence time faster without losing information, the effect of laser amplitude fluctuations is further reduced.

Combining all of the phase terms applied by the Dazzler to the input pump pulse, we have (1) the phase term imparting the time delay $\tau,(2)$ the sets of phases $\left\{\phi_{1}, \phi_{2}\right\}$ applied for phase cycling, (3) an additional $\tau$-dependent phase $\omega_{L} \tau$ for phase-locked detection, (4) a spectral phase $\psi(\omega)=\psi^{(2)}\left(\omega-\omega_{0}\right)^{2}+\psi^{(3)}\left(\omega-\omega_{0}\right)^{3}+\ldots$ to finely adjust pulse compression, and (5) an amplitude mask $A(\omega)$ with bandwidth limited by the Dazzler crystal thickness and the GDD applied in $\psi(\omega)$. The pump field at the output of the Dazzler is the input $\boldsymbol{E}_{\text {in }}(\omega)$ times the total phase function:

$$
\boldsymbol{E}_{\text {out }}(\omega)=\boldsymbol{E}_{\text {in }}(\omega) A(\omega)\left[e^{i \phi_{1}}+e^{i \phi_{2}} e^{i\left(\omega-\omega_{L}\right) \tau}\right] e^{i \psi(\omega)}
$$

The phase cycling suppresses terms from individual pump and probe beams scattered in the phase-matched direction, but there can also be scatter contributuions from interference between pairs of pump pulses. These terms do depend on both phases $\phi_{1}$ and $\phi_{2}$, and can therefore appear as artifacts in the rephasing and nonrephasing signals even after phasecycling. To suppress these terms, a shutter is placed in the probe beam path before the diffractive optic, which blocks both the probe and local oscillator. Pump scatter frames are acquired for each of the 240 Dazzler waveforms, with a shutter open duty cycle of $90 \%$. The 
pump scatter frames for each corresponding waveform are subtracted from the raw spectra before performing interferometry and phase cycling.

\subsubsection{Spectral Interferometry and Phasing}

The amplitude and phase of signals propagating in the $-\vec{k}_{1}+\vec{k}_{2}+\vec{k}_{3}$ direction are extracted using Fourier transform spectral interferometry [98, 99]. The attenuated local oscillator beam described in Figure 2.6 co-propagates with the phase-matched signals, and the sum of the two fields is detected with a spectrometer. The spectrometer measures the intensity $I(\omega)$ of the signal field $E_{s i g}$ plus the LO field $E_{L O}$, which delayed by $\tau \approx 800 \mathrm{fs}$ (different from the coherence time $\tau$ ) relative to the signal:

$$
\begin{aligned}
I(\omega) & =\left|E_{\text {sig }}(\omega)+E_{L O}(\omega) e^{i \omega \tau}\right|^{2} \\
& =\left|E_{\text {sig }}\right|^{2}+\left|E_{L O}\right|^{2}+E_{\text {sig }}^{*} E_{L O} e^{i \omega \tau}+E_{\text {sig }} E_{L O}^{*} e^{-i \omega \tau}
\end{aligned}
$$

The inverse Fourier transform of $I(\omega)$ gives a sum of convolutions in the time domain [99]:

$$
\begin{aligned}
\mathrm{FT}^{-1}\{I(\omega)\}= & E_{\text {sig }}^{*}(-t) * E_{\text {sig }}(t)+E_{L O}^{*}(-t) * E_{L O}(t) \\
& +f(t-\tau)+f(-t-\tau)^{*}
\end{aligned}
$$

where the two interference terms $f(t):=E_{\text {sig }}^{*}(-t) * E_{L O}(t)$ are centered at $t= \pm \tau$, and * denotes a convolution. The $E_{\text {sig }}$ and $E_{L O}$ autocorrelation terms are centered at $t=0$ with widths inversely proportional to their frequency domain bandwidths. By choosing a sufficiently large probe/LO delay, the interference terms can be isolated from the DC peaks by multiplying $I(t)$ with a window function centered at $t= \pm \tau$. Fourier transforming the isolated term $f(t-\tau)$ gives $f(\omega)=E_{\text {sig }}(\omega) E_{L O}^{*}(\omega)$. The amplitude and phase of the signal field can then be obtained by dividing by the local oscillator field. We use the Fourier transform of the DC term in Equation 2.17 to estimate the amplitude $\left|E_{L O}(\omega)\right| \approx \sqrt{I_{L O}(\omega)}$ under the assumption that $\left|E_{\text {sig }}\right|^{2}<<\left|E_{L O}\right|^{2}$.

$$
E_{\text {sig }}(\omega)=\frac{E_{\text {sig }} E_{L O}^{*}}{\sqrt{I_{L O}(\omega)}}
$$


The choice of window function can make a significant impact on the resulting $2 \mathrm{D}$ spectrum. Multiplying the signal by a window in the spectral interferometry time domain amounts to a convolution of the signal and window in the frequency domain, with the widths of the window functions inversely proportional in the two domains. Using a narrow window in time can be helpful for noise filtering, but causes blurring of the $2 \mathrm{D}$ spectrum along the detection axis. Using a window function with sharp edges leads to Fourier ringing artifacts in the resulting spectrum, which becomes significant for 2DES at low temperatures where spectral resolution is a limiting factor.

Since we do not know the absolute phase of the local oscillator, Equation 2.18 gives the amplitude and phase of $E_{\text {sig }}(\omega)$ times an unknown relative phase between the signal and LO. The "global phasing" procedure to determine this unknown phase involves comparing the 2D spectrum at $\tau=0$ to an independent transient absorption measurement acquired under identical excitation conditions. The projection of the 2DES spectrum along the detection axis is related to the transient grating signal $\boldsymbol{E}_{T G}$ at a given population time by the projection slice theorem $[87,100]$ :

$$
\int_{-\infty}^{\infty} \boldsymbol{S}_{2 D}\left(\omega_{1}, T, \omega_{3}\right) d \omega_{1}=\boldsymbol{S}_{T G}\left(T, \omega_{3}\right) n\left(\omega_{3}\right) /\left|\omega_{3}\right|
$$

The transient grating signal obtained from the 2DES measurement has the same relative phase as the 2D spectrum, and can be obtained by taking the sum of rephasing and nonrephasing signals at $\tau=0$. The unknown spectral phase $\phi\left(\omega_{3}\right)$ is that which minimizes the residual between the TG spectrum $\boldsymbol{S}_{T G}\left(T, \omega_{3}\right)$ and transient absorption spectrum $\boldsymbol{S}_{T A}\left(T, \omega_{3}\right)$ at the same population time $T$ [94]:

$$
\min \left|\boldsymbol{S}_{T G}\left(\omega_{3}\right) e^{i \phi\left(\omega_{3}\right)}-\alpha \boldsymbol{S}_{T A}\left(\omega_{3}\right)\right|^{2}
$$

The spectral phase assumed to be a second-order polynomial:

$$
\phi(\omega)=\phi_{0}+\phi_{1}\left(\omega-\omega_{0}\right)+\phi_{2}\left(\omega-\omega_{0}\right)^{2}
$$

The $\phi_{1}$ and $\phi_{2}$ terms are the relative time delay and GDD, respectively, imparted by the different propagation paths of the probe and local oscillator through the sample and the 
delay plate. The scaling factor $\alpha$ and phase coefficients are found using a nonlinear least squares algorithm. The transient absorption spectrum is easily acquired before each $2 \mathrm{D}$ scan by blocking the probe and one of the pump beams, and removing the delay plate. The local oscillator becomes the probe for the transient absorption measurement, and the measurement is done under identical excitation conditions at the same sample position.

\subsubsection{BRC 2DES Experiments}

In order to obtain accurate time-dependent spectra for the kinetic analysis presented in Chapters 3 and 4, it is important to ensure that each laser shot excites an ensemble of samples with the same initial conditions. For wild-type BRCs, this presents an additional challenge due to the long lifetime of the charge-separated $\mathrm{P}^{+} \mathrm{Q}_{A}^{-}$state, which takes on the order of $100 \mathrm{~ms}$ to decay to the ground state [101]. For high repetition rate experiments, $\mathrm{P}^{+} \mathrm{Q}_{A}^{-}$states will slowly build up with successive laser shots, causing spurious time-dependent trends in the data, as well as a time-independent signal contributuions from an equilibrium concentration of $\mathrm{P}^{+} \mathrm{Q}_{A}^{-}$.

There are several approaches for dealing with the build-up of long-lived states in high repetition rate $\mathrm{BRC}$ experiments. The simplest option is to reduce the repetition rate to the order of $10 \mathrm{~Hz}$, so that the majority of excited samples have decayed to the ground state before the next laser shot [53, 102-104]. This greatly increases the acquisition time, which is already signficant for transient absorption experiments, and becomes outright unfeasible for 2DES. A 2D data set with comparable averaging to the one presented in Chapter 4

would take approximately 2.5 days to acquire at $10 \mathrm{~Hz}$, over which time small changes in laser stability, amplitude and spectrum cause significant noise and distortions in the 2D spectrum.

Often rotating sample cells are used so that each laser shot hits a different, unexcited spot on the sample [105-108]. This approach works well for transient absorption experiments, but 2DES is much more sensitive to scattered light in general, and especially time-dependent scatter which produces Fourier transform artifacts on the excitation axis. Rotating or trans- 
lating the sample is also particularly challenging for experiments at cryogenic temperatures. Similarly, room temperature samples in solution can run through a flow cell with a high enough flow rate to replenish the sample for each laser shot $[28,61,109-111]$. This approach presents similar challenges with time-dependent light scattering, and cannot be done at cryogenic temperatures.

In order to facilitate high-repetition rate experiments at cryogenic temperatures without the use of cumbersome, noise-inducing cryostat rotation scheme, we chose to study two BRC mutants which do not form the long-lived $\mathrm{P}^{+} \mathrm{Q}_{A}^{-}$state. The first mutation, W(M250)V [23], blocks the binding of $\mathrm{Q}_{A}$ but is otherwise unperturbed, allowing us to study the energy transfer dynamics, and the charge separation sequence up to the formation of $\mathrm{P}^{+} \mathrm{H}_{A}^{-}$, which decays to the ground state on a timescale of 15ns [101]. The second mutant, DLL, lacks the A-branch bacteriopheophytin, and does not exhibit any charge separation [36, 59]. Both the W(M250)V and DLL samples were dissolved in a 50/50 buffer/glycerol mixture, and loaded into a $380 \mu \mathrm{m}$ sample cell in a liquid nitrogen cooled cryostat. The sample concentrations were such that the room-temperature P-band peak had an optical density of 0.3 with a $380 \mu \mathrm{m}$ path length.

Another potential source of error is signal contributions from samples with multiple excitations. If the intensity of the pump pulse is sufficiently high, the probability that a given RC absorpbs two pump photons becomes significant, and some such interactions (which are now fifth-order) phase-match in the direction of the measured signal. Such interactions might include, for instance, simultaneously exciting both $\mathrm{B}_{A}$ and $\mathrm{B}_{B}$ or other combinations of chromophores, which would generate signal contributions with dynamics that are not well-represented by a linear kinetic model. To avoid double excitations, transient absorption studies typically use pump pulse energies low enough to excite $5 \%$ or less of the RCs within the pulse overlap region $[49,61,106,107]$, although some earlier studies reported considerably higher excitation probabilities of $>10 \%[77,112]$. 
For the W(M250)V 2DES experiments, with 20nJ pump pulses and $200 \mu \mathrm{m} 1 / \mathrm{e}^{2}$ spot sizes, the pump absorption probability is on the high end of values reported in the literature, with a maximum probability of $30 \%$ at the center of the pump pulse based on beam profile measurements. Based on the $77 \mathrm{~K}$ optical density of the sample and measured pump spectrum, $55 \%$ of pump photons are absorbed by the sample. From the measured pump beam profile and the sample concentration, we estimate from the spatially dependent excitation probability with a Poisson distribution. Averaging over the pump spot size, $\% 7$ of the excited RCs have more than one excitation. As a control experiment, we performed a series of kinetic scans with narrower-bandwidth (20-30nm), low energy $(<4 \mathrm{~nJ})$ pump pulses, and compared the apparent rates to the full bandwidth 2DES measurements. The fast dynamics on the order of tens of femtoseconds were slightly altered due to the longer pulse durations of the narrow-bandwidth pump pulses, but the 100fs and picosecond timescales gave similar results.

2DES spectra for the W(M250)V mutant BRC were recorded at $77 \mathrm{~K}$ for 150 population times ranging from -20fs to 1ns. Population times were spaced hyperbolically with respect to $T=0$, so that the sub-picosecond dynamics were sampled more finely, and the spacing increases continuously over the scan range. The coherence time was scanned to $600 \mathrm{fs}$ with $8 \mathrm{fs}$ spacing, acquired in the rotating frame as described in Section 2.2. Each scan was acquired with pump polarizations parallel, perpendicular, and at the magic angle with respect to the probe polarization. The measured pump and probe pulse energies at the sample position were $14 \mathrm{~nJ}$ and $19 \mathrm{~nJ}$, respectively, and pulses were focused to a $1 / e^{2}$ spot size of $\approx 200 \mu \mathrm{m}$. The pump and probe pulse durations estimated from the SPEAR [113] and MIIPS [114] pulse compression algorithms were $12.8 \mathrm{fs}$ and $10.0 \mathrm{fs}$, respectively. Figure 2.8 shows the measured pump and probe spectra in relation to the $\mathrm{W}(\mathrm{M} 250) \mathrm{V}$ and DLL linear absorption spectra. The DLL absorption spectrum has a reduced $\mathrm{H}$ peak due to the removal of $\mathrm{H}_{A}$, as well as a blue shift of the $\mathrm{P}$ band. The fringes on the pump and probe spectra are due to etaloning effects in the spectrometer CCD, and are filtered out of the 2DES data when the signal is 
extracted using spectral interferometry. The DLL 2DES experiments were performed under similar excitation conditions as W(M250)V, with 24nJ pump and 29nJ probe pulses, and similar spot sizes.

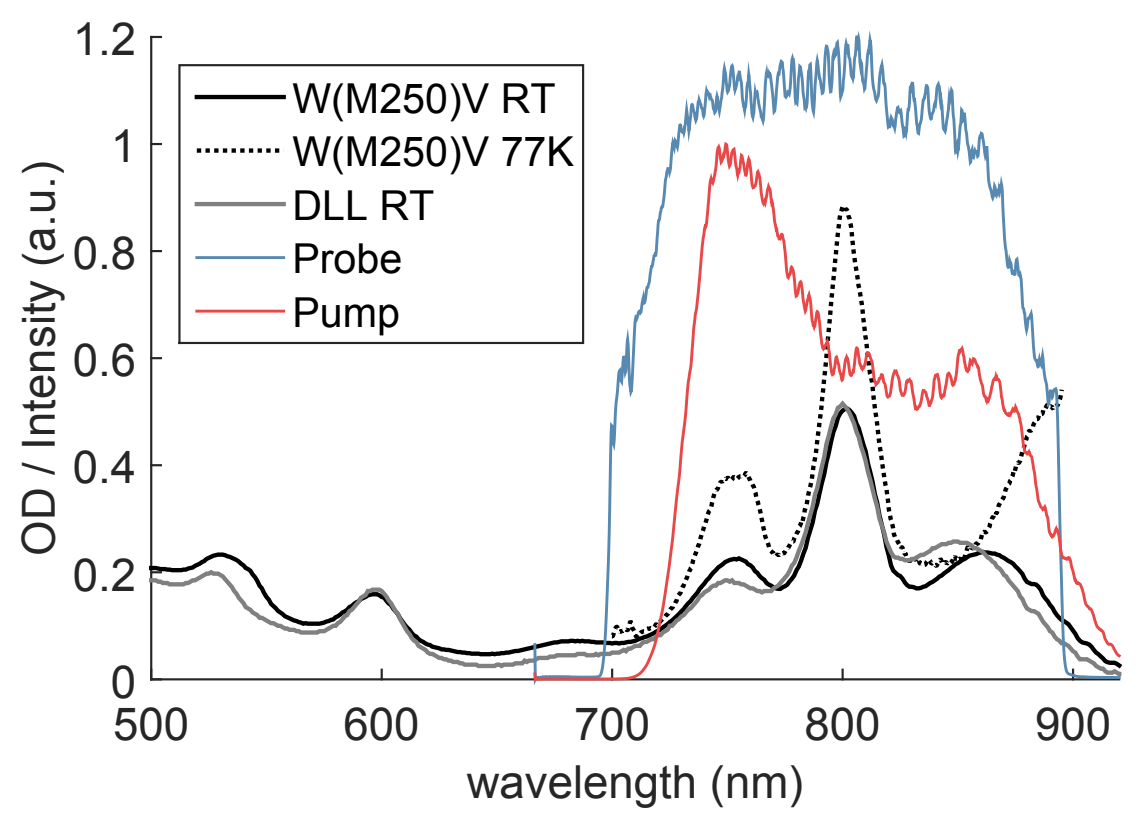

Figure 2.8: Linear absorption spectra at $298 \mathrm{~K}$ and $77 \mathrm{~K}$, pump and probe spectra used for W(M250)V and DLL 2DES experiments.

\subsection{Optical Parametric Amplifiers}

One of the challenges of doing broad-bandwidth spectroscopy in the 700-900nm spectral range is generating laser pulses with a suitably flat spectrum, well-behaved spectral phase, and high stability. Supercontinuum generation is often used in transient absorption spectroscopy as a broadband probe source [48, 57, 107, 108, 115-117], and has also been applied to 2DES [118]. White light pulses are typically generated using the 800nm output of a Ti:sapphire amplifier, making the spectrum and spectral phase highly structured and unstable near 800nm. This instability also makes the white light spectra unsuitable for amplification with an OPA. In order to generate spectrally smooth and stable pulses spanning the BRC Qy band using a Ti:sapphire pump source, we built a degenerate optical parametric amplifier (DOPA) based on the design by Siddiqui et al. [119], which uses the 
output of a near IR OPA to generate the continuum seed. We also routinely use noncollinear optical parametric amplifiers (NOPAs) [120] to generate broad tunable pulses spanning the visible spectrum, which enable us to extend our accessible spectral range to probe the bacteriochlorophyll anion band at 650nm [5, 43, 60].

OPAs are commonly used as light sources for nonlinear spectroscopy applications, and have largely replaced dye lasers as the standard for tunable femtosecond sources in the visible and near-IR [121]. They operate using difference frequency mixing, a second-order nonlinear mixing process described by the $\chi^{(2)}$ term in Equation 2.1, to transfer energy from a higher-frequency "pump" pulse at frequency $\omega_{p}$ to "signal" $\left(\omega_{s}\right)$ and "idler" $\left(\omega_{i}\right)$ pulses, where $\omega_{p}>\omega_{s}>\omega_{i}$. The high-intensity pump pulse with field $E_{p}(t)$ and lower intensity signal pulse $E_{s}(t)$ are focused into a birefringent crystal with a $\chi^{(2)}$ response. Defining the input fields analogously to the $\chi^{(3)}$ case in Equation 2.6:

$$
\begin{aligned}
\boldsymbol{E}_{\boldsymbol{j}}(t, \vec{r}) & =E_{j}(t) e^{i\left(\vec{k}_{j} \cdot \vec{r}-\omega_{j} t\right)} \hat{e}_{j}+c . c . \\
\boldsymbol{E}(t, \vec{r}) & =\boldsymbol{E}_{p}+\boldsymbol{E}_{s}
\end{aligned}
$$

The second-order polarization $P_{i}^{(2)}=\epsilon_{0} \chi_{i j k}^{(2)} E_{j} E_{k}$ oscillates in response to the the input fields, and acts as a source term in Equation 2.3, as in the four-wave mixing case, radiating an electric field with cross-terms between the pump and signal fields. The difference frequency mixing term oscillating at $\omega_{i}=\omega_{p}-\omega_{s}$ with spatial dependence $e^{i\left(\vec{k}_{p}-\vec{k}_{s}\right) \cdot \vec{r}}$ is responsible for parametric amplification. The generated idler field satsifies energy and momentum conservation:

$$
\begin{aligned}
& \omega_{p}=\omega_{s}+\omega_{i} \\
& \vec{k}_{p}=\vec{k}_{s}+\vec{k}_{i}
\end{aligned}
$$

The refractive indices of the pump, signal and idler are different as they propagate through an isotropic crystal, with $n_{p}>n_{s}>n_{i}$ in the normal dispersion region. The difference in group velocities between the three propagating fields leads to a mismatch in the phase 
between the amplified signals generated at different $z$-positions in the crystal. Defining the phase mismatch $\Delta k$ as:

$$
\begin{aligned}
\Delta k\left(\omega_{s}\right) & =k_{p}-k_{s}\left(\omega_{s}\right)-k_{i}\left(\omega_{s}\right) \\
& =\frac{n\left(\omega_{p}\right) \omega_{p}}{c}-\frac{n\left(\omega_{s}\right) \omega_{s}}{c}-\frac{n\left(\omega_{i}\right) \omega_{i}}{c}
\end{aligned}
$$

where $\omega_{i}=\omega_{p}-\omega_{s}$. In order to maximize the signal bandwidth that adds constructively as it propagates through the crystal, we must minimize $\Delta k$ over a range of signal frequencies, which amounts to minimizing its Taylor expansion with respect to the signal frequency about a central frequency $\omega_{0}$ :

$$
\left.\Delta k\left(\omega_{s}\right) \approx \Delta k_{0}\right|_{\omega_{s}=\omega_{0}}+\left.\frac{\partial \Delta k}{\partial \omega_{s}}\right|_{\omega_{s}=\omega_{0}}\left(\omega_{s}-\omega_{0}\right)+\left.\frac{1}{2} \frac{\partial^{2} \Delta k}{\partial^{2} \omega_{1}}\right|_{\omega_{s}=\omega_{0}}\left(\omega_{s}-\omega_{0}\right)^{2}+\ldots
$$

Minimizing the first term of Equation 2.25 is accomplished by using the birefringence of the crystal to control the refractive indices of the pump, signal and/or idler. For a birefringent crystal, the refractive index is given by the projection of the polarization vector of the propagating wave onto the refractive index ellipsoid. For a uniaxial crystal, two of the three indices are degenerate, defined as the ordinary index $n_{o}$, and the unique axis is the extraordinary index $n_{e}$ [122]. If the $n_{e}$ axis is oriented in the plane defined by the polarization $\hat{e}$ and the propagation direction $\vec{k}$ of an incident wave, then the refractive index depends on the angle $\theta$ between $\vec{k}$ and $n_{e}$ :

$$
\frac{1}{n_{e}^{2}(\theta)}=\frac{\cos ^{2} \theta}{n_{o}^{2}}+\frac{\sin ^{2} \theta}{n_{e}^{2}}
$$

The index $n_{e}(\theta)$ ranges from $n_{o}$ to $n_{e}$, both of which also depend on frequency. The $\beta$-Barium Borate (BBO) crystals used in the DOPA and NOPAs is negative uniaxial, meaning $n_{e}<n_{o}$. So, in order to satisfy the condition $n\left(\omega_{p}\right) \omega_{p}-n\left(\omega_{s}\right) \omega_{s}-n\left(\omega_{i}\right) \omega_{i}$ in the normal dispersion region, we must decrease $n\left(\omega_{p}\right)$ by some amount, by projecting it onto the lower-index $n_{e}$ axis so that $n_{p}=n_{e}\left(\theta, \omega_{p}\right)$. If the signal and idler are polarized perpendicular to the pump (known as Type I phase-matching), then $n_{s}=n_{o}\left(\omega_{s}\right)$ and $n_{i}=n_{i}\left(\omega_{s}\right)$. The phase-matching 
condition $\Delta k=0$ can be satisfied for a given $\omega_{s}$ by finding $\theta$ such that:

$$
\Delta k_{0}=2 \pi c\left(\frac{n_{e}\left(\lambda_{p}, \theta\right)}{\lambda_{p}}-\frac{n_{o}\left(\lambda_{s}\right)}{\lambda_{s}}-\frac{n_{o}\left(\lambda_{i}\right)}{\lambda_{i}}\right)=0
$$

The frequency dependence of $n_{o}$ and $n_{e}$ is described by the Sellmeier equations (for BBO, see [123]), usually presented as a function of wavelength. For a 400nm pump and 800nm signal and idler, the phase-matching angle is $29.2^{\circ}$ (not coincidentially the same angle for phase-matched SHG of 800nm, since degenerate difference-frequency generation and SHG are inverse processes).

The phase-matching angle sets the zeroth-order term of the $\Delta k$ expansion in Equation 2.25 to zero at a specified signal frequency $\omega_{s}=\omega_{0}$. For the first-order term, we can evaluate the partial derivative, with $\omega_{i}=\omega_{p}-\omega_{s}$, and $\omega_{p}$ assumed to be constant:

$$
\begin{aligned}
\frac{\partial \Delta k}{\partial \omega_{s}} & =\frac{\partial}{\partial \omega_{s}}\left(k_{p}-k_{s}-k_{i}\right) \\
& =-\frac{\partial k_{s}}{\partial \omega_{s}}-\frac{\partial k_{i}}{\partial \omega_{s}} \\
& =-\left(\frac{1}{v_{g s}}-\frac{1}{v_{g i}}\right)
\end{aligned}
$$

So the first-order term in $\Delta k$ is proportional to the difference of the inverse group velocities $v_{g}=\partial \omega / \partial k$ of the signal and idler evaluated at the phase-matched frequency $\omega_{s}=\omega_{0}$. Both the DOPA and NOPA maximize the amplification bandwidth by minimizing the difference between $v_{g s}$ and $v_{g i}$. When the signal and idler are degenerate (i.e. $\omega_{s}=\omega_{i}, \vec{k}_{s}=\vec{k}_{i}$, and $\hat{e}_{s}=\hat{e}_{i}$ ), they must have the same group velocities, and this condition is met automatically (this only works for Type I phase-matching). In that case, the bandwidth-limiting factor is the second-order term of Equation 2.25, which turns out to be the sum of the group velocity dispersions of the signal and idler.

For the non-degenerate case, the crossing angle between the pump and signal beams provides an additional degree of freedom to eliminate the first-order $\Delta k$ term. For the noncollinear beam geometry shown in Figure 2.9, the new phase-matching condition requires that the vector components of the phase mismatch parallel and perpendicular to $\vec{k}_{p}$ be zero: 


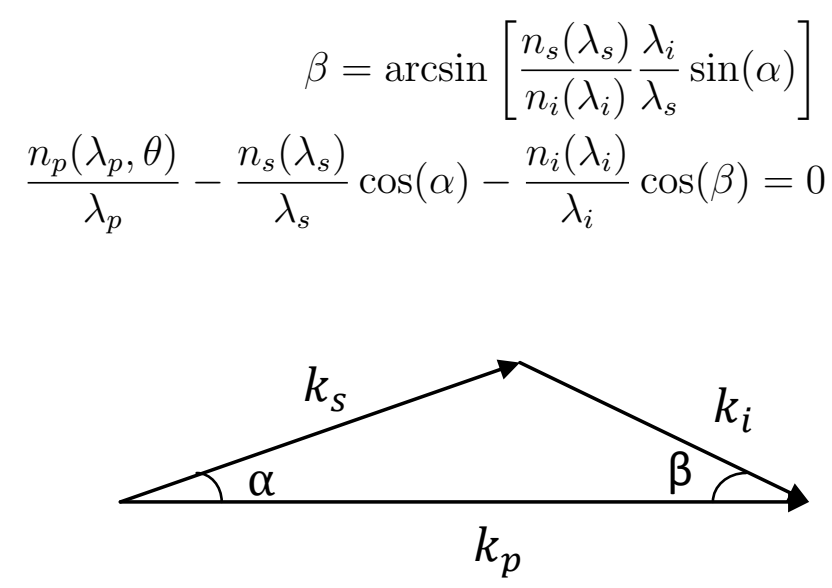

Figure 2.9: Noncollinear pump, signal, and idler wavevectors

For each noncollinear angle $\alpha$ and signal wavelength $\lambda_{s}$, there is an idler angle $\beta$ which satisfies phase-matching perpendicular to $\vec{k}_{p}$ (Equation 2.28), and one can find the BBO angle $\theta$ satisfying the parallel phase-matching condition (Equation 2.28). The optimal $\alpha$ minimizes the partial derivative of Equation 2.28 with respect to $\omega_{s}$ at the desired signal frequency. $\alpha$ is the internal crossing angle inside the BBO. For significant noncollinear angles, one must account for the refraction of $\vec{k}_{s}$ and $\vec{k}_{p}$ at the BBO surface to find the external crossing angle. Note that the DOPA phase-matching conditions are the special case of the NOPA conditions where $\lambda_{s}=\lambda_{i}$, so Equation 2.28 reduces to $\alpha=\beta$, and $\alpha=0$ is the optimal noncollinear angle for signal/idler group velocity matching.

Figure 2.10 shows the layout of the DOPA used as the light source for our 2DES experiments. A portion of the $800 \mathrm{~nm}$ output from a Ti:sapphire regenerative amplifier pumps a two-stage IR OPA generating $5 \mu \mathrm{J}$ pulses tunable from $1.2 \mu \mathrm{m}$ to $1.6 \mu \mathrm{m}$. The $1.2 \mu \mathrm{m}$ output of the OPA focuses onto a sapphire plate to generate a stable continuum spanning the 600-1000nm amplification region. The remaining portion of the Ti:sapphire beam generates the 400nm pump for the final degenerate amplification stage. The pump and seed beams are focused onto a BBO crystals $\left(1 \mathrm{~mm}, 29.2^{\circ}\right)$ with a small crossing angle $\left(1^{\circ}\right)$, which is necessary to spatially separate the signal from the idler. Since the DOPA uses the type 1 phase-matching configuration to achieve broad bandwidth amplification, the signal and idler 
share the same polarization and cannot be separated by polarizer. The DOPA outputs $8 \mu \mathrm{J}$ pulses spanning $680-920 \mathrm{~nm}$, which are used for the pump and probe pulses in the 2DES setup in Figure 2.5.

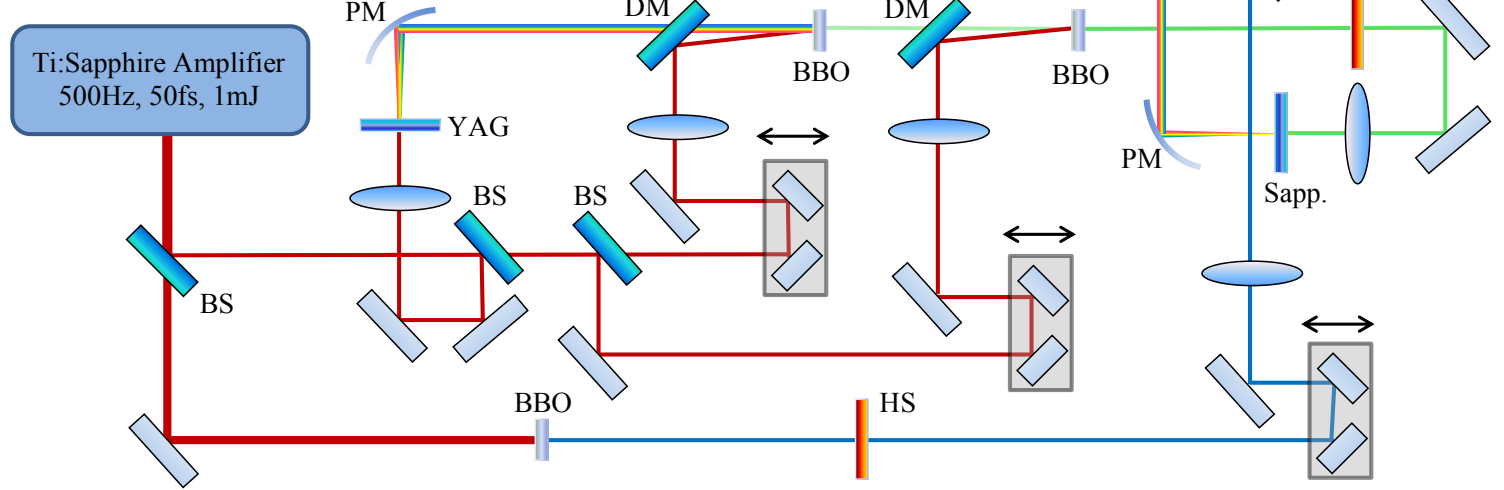

Figure 2.10: Schematic of Degenerate OPA design. BS: beam splitter, BBO: $\beta$-barium borate crystal, YAG: yttrium aluminum garnet crystal, PM: off-axis parabolic mirror, DM: dichroic mirror, LPF: long-pass filter $(1 \mu \mathrm{m})$, HS: harmonic separater $(400 \mathrm{~nm} / 800 \mathrm{~nm})$ 


\section{CHAPTER 3}

\section{ANALYSIS OF TIME-RESOLVED SPECTRA}

One of the greatest challenges in the application of time-resolved spectroscopy to complicated, multi-chromophoric systems such as photosynthetic light harvesting complexes is extracting useful, quantative information from the transient spectra. Generally, the absorption bands of the constituent chromophores overlap, so that exciting an ensemble of samples at a given wavelength results in a superposition of signals from different initial conditions. Furthermore, the spectral signatures associated with different product states often overlap as well, and can vary in amplitude by orders of magnitude. States which are optically dark, such as charge transfer states, or states with relatively weak transition dipole moments, can be obscured by stronger stimulated emission and ground state bleach signals. Short-lived intermediate states such as the $P^{+} B_{A}^{-}$state in BRCs can also be dominated by signals from product states with much higher concentrations. All of these overlapping effects combine to make it difficult (or in some cases impossible) to uniquely determine the transfer rates between different states, and their associated spectral signatures, especially for systems with multiple branched energy transfer pathways.

Most time-resolved spectroscopy experiments on photosynthetic systems have been done in solution, either at room temperature in a flow cell, or in a glassy mixture at 77K. This presents an additional challenge in that the samples are randomly oriented, so the measured signal is a rotational average of all sample orientations. The probability of a given state interacting with a pump or probe pulse depends on the projection of the pulse polarization in the direction of the transition dipole moment. In the case of parallel pump and probe 
polarizations, this results in the detected signal from a product state being weighted by the cosine of the angle between the transition dipole moments of the initial state and the product state. This presents another challenge for kinetic fitting, because the amplitude of the product state signal depends on which state was initially excited in each sample.

\subsection{Kinetic Fitting of Transient Spectra}

In the case of transient absorption data, the measured difference spectrum $S(\lambda, t)$ can be represented as a linear combination of basis spectra $f_{i}(\lambda)$ with time-varying concentrations $c_{i}(t):$

$$
S(\lambda, t)=\sum_{i=1}^{n} c_{i}(t) f_{i}(\lambda)
$$

The goal is to find a model with $n$ states which correspond to real chemical species of the system, each with a difference spectrum $f_{i}(\lambda)$ associated with it. The concentrations $c_{i}(t)$ are determined by the kinetic model and initial conditions chosen. Generally, a linear kinetic scheme is assumed, in which case the concentrations are governed by a system of $n$ first-order differential equations [73]:

$$
\frac{d}{d t} c_{i}(t)=\sum_{j=1}^{n} K_{i j} c_{j}(t)+a_{i}(t)
$$

where the matrix elements $K_{i j}$ define the rate constants for population transfer between different species. The diagonal elements $K_{i i}$ are the rate constants for decay of each compartment $i$ to the ground state. $a_{i}(t)$ defines the initial population of each species, and is determined by the excitation conditions of the experiment, including the temporal profile of the pump pulse, and the time-zero absorbances of each species [124].

Equation 3.2 has a general analytic solution [73] given by:

$$
\vec{c}(t)=e^{K t} * \vec{a}(t)
$$

where $*$ denotes convolution, and $e^{K t}=1+K t+(K t)^{2} / 2+\ldots$ is a matrix exponential. In the impulsive limit where $\vec{a}(t)=\vec{a}_{0} \delta(t)$ (i.e. the pump pulse is much shorter than the measured 
system dyanmics), Equation 3.3 is simply a sum of exponentials, with time constants $\lambda_{i}$ being the eigenvalues of $\mathrm{K}$. By diagonalizing $K$, the matrix exponential can be evaluated:

$$
\begin{aligned}
K & =U \Lambda U^{-1} \\
K^{n} & =U \Lambda^{n} U^{-1} \\
e^{K t} & =U e^{\Lambda t} U^{-1}
\end{aligned}
$$

where $\Lambda$ is a diagonal matrix comprised of the eigenvalues of $K$, and $U$ is a unitary matrix with columns being the eigenvectors of K. Then, in the impulsive limit, Equation 3.3 becomes:

$$
\vec{c}(t)=U \operatorname{diag}\left(e^{-\lambda_{1} t}, \ldots, e^{-\lambda_{n} t}\right) U^{-1} \vec{a}_{0}
$$

In the more general case where the temporal profile of $\vec{a}(t)=\vec{a}_{0} I_{p}(t)$ is the pump pulse intensity, the exponentials in Equation 3.4 are convolved with $I_{p}(t)$.

For a given matrix of rate constants $K$ and excitation conditions $\vec{a}(t)$, the time-dependent concentrations can be computed with Equation 3.4 for arbitrary t>0. Returning to Equation 3.1, we now want to find the basis spectra $f_{i}(\lambda)$ which best reconstruct the measured spectrum $S(\lambda, t)$. Assuming the signal was sampled at $m$ time points and $p$ wavelengths, and using an $n$-compartment model, Equation 3.1 can be written as a matrix equation $[74,125]$ :

$$
\begin{gathered}
\mathbf{S}_{(m \times p)}=\mathbf{C}_{(m \times n)} \mathbf{F}_{(n \times p)} \\
{\left[\begin{array}{ccc}
S\left(\lambda_{1}, t_{1}\right) & \cdots & S\left(\lambda_{p}, t_{1}\right) \\
\vdots & \ddots & \vdots \\
S\left(\lambda_{1}, t_{m}\right) & \cdots & S\left(\lambda_{p}, t_{m}\right)
\end{array}\right]=\left[\begin{array}{ccc}
c_{1}\left(t_{1}\right) & \cdots & c_{n}\left(t_{1}\right) \\
\vdots & \ddots & \vdots \\
c_{1}\left(t_{m}\right) & \cdots & c_{n}\left(t_{m}\right)
\end{array}\right]\left[\begin{array}{ccc}
f_{1}\left(\lambda_{1}\right) & \cdots & f_{1}\left(\lambda_{p}\right) \\
\vdots & \ddots & \vdots \\
f_{n}\left(\lambda_{1}\right) & \cdots & f_{n}\left(\lambda_{p}\right)
\end{array}\right]}
\end{gathered}
$$

where $t=\left\{t_{1}, \ldots, t_{m}\right\}$ and $\lambda=\left\{\lambda_{1}, \ldots, \lambda_{p}\right\}$ are the sampled time and wavelength points, respectively. The columns of $\mathbf{S}$ are the measured time traces at each wavelength, the columns of $\mathbf{C}$ are the concentrations of each compartment, given by Equation 3.4 and evaluated at $t=$ $\left\{t_{1}, \ldots, t_{m}\right\}$, and the rows of $\mathbf{F}$ are the difference spectra associated with each compartment, sometimes called Species Associated Difference Spectra (SADS). 
There is some ambiguity in the literature regarding the use of the terms "compartments", "species" and "states". Generally, global and target analysis methods assume the linear compartmental model defined by Equations 3.1 and 3.2, with different assumptions on the form of $K$. As I will show below, a given model can fit the data using compartments which do not correspond to actual states or species of the system, but rather are linear combinations of them $[73,124]$. Throughout this chapter, I refer to "compartments" as the arbitrary mathematical constructs assumed by a particular model. The compartments can only safely be called "species" or "states" when the chosen model reflects the actual physics of the system.

Generally, Equation 3.1 will have $m$ time-points, and $n<m$ compartments, so for each wavelength there are $m$ equations and $n$ unknown values $f_{n}(\lambda)$, making the system overdetermined. We then want the least-squares solutions for $\vec{f}(\lambda)$ at each wavelength, which can easily be computed using the pseudo-inverse of the concentration matrix $\mathbf{C}$ :

$$
\mathbf{F}=\mathbf{C}^{+} \mathbf{S}=\left(\mathbf{C}^{T} \mathbf{C}\right)^{-1} \mathbf{C}^{T} \mathbf{S}
$$

Given a set of rate constants $K$ and excitation conditions $\vec{a}(t)$, this gives the basis spectra $\mathbf{F}$ which minimize the Frobenius norm (i.e. minimize the sum of the squares of each matrix element) of the error, $\|\mathbf{S}-\mathbf{C F}\|_{F}$. Finding the optimal rate constants for a given $n$-compartment model, however, requires a nonlinear search method. A variety of methods have been employed to search for the global optimum with respect to these nonlinear parameters, including the Levenberg-Marquardt algorithm [67, 124, 126, 127], Adaptive Random Search $[64,128]$, and other variations of iterative gradient descent methods such as Trust Region method [129]. For an overview of methods for solving nonlinear least squares problems, see [130].

The process of separating the parameters in a nonlinear data fitting problem (i.e. Equation 3.1) into nonlinear parameters, and linear parameters which depend on the nonlinear ones, is known as the Variable Projection method [131]. Variable Projection is useful in a wide variety data fitting and inverse problems in which observed data is modeled as a linear 
combination of nonlinear functions [129]:

$$
y_{i}=\sum_{j=1}^{n} a_{j} \phi_{j}\left(\boldsymbol{\alpha} ; t_{i}\right)
$$

where $y_{i}$ are observed data points, $t_{i}$ are independent variables, $\boldsymbol{\alpha}$ are nonlinear parameters of $\phi_{j}$, and $a_{j}$ are the (linear) coefficients of the functions $\phi_{j}$. The least-squares minimal parameters $(\boldsymbol{a}, \boldsymbol{\alpha})$ will minimize the residual $\|\boldsymbol{r}(\boldsymbol{a}, \boldsymbol{\alpha})\|_{2}^{2}=\|\boldsymbol{y}-\boldsymbol{\Phi}(\boldsymbol{\alpha}) \boldsymbol{a}\|_{2}^{2}$, where the columns of $\boldsymbol{\Phi}$ are $\phi_{j}$. The coefficients $\boldsymbol{a}=\boldsymbol{\Phi}(\boldsymbol{\alpha})^{+} \boldsymbol{y}$ are found using the pseudo-inverse of $\boldsymbol{\Phi}$, and the problem becomes a minimization of the residual with respect to only $\boldsymbol{\alpha}$ :

$$
\begin{aligned}
\boldsymbol{\alpha} & =\min _{\boldsymbol{\alpha}}\left\|\left(\boldsymbol{I}-\boldsymbol{\Phi}(\boldsymbol{\alpha}) \boldsymbol{\Phi}(\boldsymbol{\alpha})^{+}\right) \boldsymbol{y}\right\|_{2}^{2} \\
& =\min _{\boldsymbol{\alpha}}\left\|r_{2}(\boldsymbol{\alpha})\right\| .
\end{aligned}
$$

$r_{2}(\boldsymbol{\alpha})$ is the variable projection of $\boldsymbol{y}$, or the projection of $\boldsymbol{y}$ onto the subspace orthogonal to $\left\{\phi_{i}\right\}$ [129]. In terms of the compartmental kinetic model defined in Equation 3.1, the measurements $y_{i}$ for a given wavelength are $S(\lambda, t)$, the coefficients $a_{i}$ are the SADS for each compartment, $f_{i}(\lambda)$, and the nonlinear functions $\phi_{i}(\boldsymbol{\alpha}, t)$ are the solutions to the timedependent concentrations, given by Equation 3.4. The nonlinear variables $\boldsymbol{\alpha}$ are the matrix elements $K_{i j}$ (which are not all independent), and can also include the initial conditions $a_{i}$ in Equation 3.2 depending on what assumptions are made in the model.

\subsubsection{Exponential and Sequential Kinetic Models}

A simple kinetic fitting scheme commonly used in absorption and fluorescence spectroscopy is a sum of decaying exponentials. In this case, the rate matrix $K$ is diagonal, so the compartment concentrations in Equation 3.2 are decoupled. The unitary matrix $U$ in Equation 3.4 is the identity, and we get that $c_{i}(t)=e^{-\lambda_{i} t} * a_{i}(t)$ [73]. The amplitude of the components of $\boldsymbol{a}(t)=\boldsymbol{a}_{0} I_{p}(t)$ can arbitrarily be set to 1 , since the resulting spectra $f_{i}(\lambda)$ can absorb the normalization factors $\boldsymbol{a}_{\mathbf{0}}$. The basis spectra $f_{i}(\lambda)$ in this case are called Decay Associated Spectra (DAS), or Decay Associated Difference Spectra (DADS) in the context of transient absorption spectroscopy. 
DAS fitting does not generally yield basis spectra corresponding to actual chemical species present in the system, unless the species are actually completely decoupled. Systems exhibiting energy transfer between states do not fall into this category, and the resulting DAS will be linear combinations of the "true" species associated spectra. Still, DAS fitting can be a useful tool for analyzing transient spectra, and has been applied in many transient absorption studies on BRCs discussed in Chapter $2[49,107,110,132]$. Firstly, DAS fitting is relatively simple to implement, and only requires one to assume the number of states to include in the model. The nonlinear optimization will generally avoid local minima which can be problematic with more advanced branched kinetic models, and will repeatably converge to a set of rate constants which qualitatively describe the timescales of dynamics in the measured spectra. Likewise, the DAS components point to which parts of the measured spectrum change on a given timescale, making it useful as an initial data visualization tool.

Another relatively simple fitting scheme is an unbranched sequential model, in which each compartment populates the next compartment, with only the final compartment decaying to the ground state. The $K$ matrix in this case has nonzero elements $K_{(i+1, i)}$ for $i=1, \ldots, n-1$, and $K_{(n, n)}$. Only the first compartment is initially populated by the pump pulse. This is often a more physically meaningful model than DAS when studying systems with energy transfer and charge separation, although it will still fail to produce real SADS if there are, for instance, branched reactions or backwards reactions present. The concentrations can also be solved exactly in this case [73, 133]:

$$
\begin{aligned}
c_{i}(t) & =\sum_{j=1}^{i} b_{j i} e^{-k_{j} t} * a_{j}(t) \\
b_{j i} & =\prod_{m=1}^{i-1} k_{m} / \prod_{n-1, n \neq j}^{i}\left(k_{n}-k_{j}\right)
\end{aligned}
$$

where $k_{i}=K_{(i+1, i)}$ and $*$ is the convolution operator. 


\subsubsection{Uniqueness of Compartmental Models}

It is well-known that transient spectra do not contain enough information to uniquely constrain both the rate constants and basis spectra for a given compartmental model [64, $72,73,124]$. Suppose a least-squares optimal solution has been found for the rate constants and SADS given in Equation 3.5 using the minimization solutions described above. Then, applying any invertible operator $\boldsymbol{A}_{(n \times n)}$, Equation 3.5 can equivalently be written as [73]:

$$
\boldsymbol{S}=\boldsymbol{C} \boldsymbol{A}^{-1} \boldsymbol{A F}=\boldsymbol{C}^{\prime} \boldsymbol{F}^{\prime}
$$

So, for every kinetic scheme $\boldsymbol{C}$ and associated spectra $\boldsymbol{F}$ which reproduce the measured data, there are an infinite number of kinetic models $\boldsymbol{C}^{\prime}$ and basis spectra $\boldsymbol{F}^{\prime}$ which reproduce the data equally well. Such a transformation is a basis change to a linear combination of the compartments defined in Equation 3.2, corresponding to a new kinetic model which also satisfies the linear kinetic equations. It is straightforward to show, for example, that an arbitrary sequential model defined by rates $\boldsymbol{K}$ and initial conditions $\overrightarrow{a_{0}}(t)$ is exactly equivalent to a sum of decaying exponentials (i.e. decay associated spectra) if $\boldsymbol{K}$ is diagonalizable. Choosing $\boldsymbol{A}$ so that $\boldsymbol{A}^{-1} \boldsymbol{K} \boldsymbol{A}$ is diagonal, and the new concentrations are defined as $\vec{c}^{\prime}(t)=\boldsymbol{A}^{-1} \vec{c}(t)$, we have from Equation 3.2:

$$
\begin{aligned}
\frac{d}{d t} \vec{c}^{\prime}(t) & =\boldsymbol{A}^{-1} \boldsymbol{K} \boldsymbol{A} \vec{c}^{\prime}(t)+\boldsymbol{A}^{-1} \overrightarrow{a_{0}}(t) \\
& =\boldsymbol{K}^{\prime} \vec{c}^{\prime}(t)+{\overrightarrow{a_{0}}}^{\prime}(t)
\end{aligned}
$$

which has the solution

$$
\begin{aligned}
\vec{c}^{\prime}(t) & =e^{\boldsymbol{K}^{\prime} t} *{\overrightarrow{a_{0}}}^{\prime}(t) \\
& =\boldsymbol{A}^{-1} \vec{c}(t)
\end{aligned}
$$

The $\vec{c}^{\prime}(t)$ term becomes the transformed concentration matrix $\boldsymbol{C A}^{-1}=\boldsymbol{C}^{\prime}$ in Equation 3.11. The corresponding DAS $\boldsymbol{F}^{\prime}$ will then be linear combinations of the SADS $\boldsymbol{F}$. 
So, for any diagonalizable matrix $\boldsymbol{K}$, there is an equivalent DAS model, and the concentrations and SADS for the compartmental model are linear combinations of decaying exponentials and their corresponding DAS. There are an infinite number of such invertible transformations $\boldsymbol{A}$ which result in equivalent linear models satisfying Equation 3.2.

For the special case of the unbranched sequential model (Equation 3.9), if one assumes $N$-compartments with rate constants $k_{i}$, the $\boldsymbol{K}$ matrix is:

$$
\boldsymbol{K}=\left[\begin{array}{cccccc}
-k_{1} & 0 & 0 & \cdots & 0 & 0 \\
k_{1} & -k_{2} & 0 & \cdots & 0 & 0 \\
\vdots & \vdots & \vdots & \ddots & \vdots & \vdots \\
0 & 0 & 0 & \cdots & k_{N-1} & -k_{N}
\end{array}\right]
$$

which has eigenvalues $\left\{\lambda_{i}\right\}=\left\{-k_{i}\right\}$. Evaluating the matrix exponential, the concentrations in the eigenbasis of $\boldsymbol{K}$ are the decaying exponentials of Equation 3.9, with the initial conditions of each compartment determined by the eigenvectors of $\boldsymbol{K}$. Therefore, if we arrive at some optimal set of rates $\left\{k_{i}\right\}$ for the sequential model, any permutation of those same rates will give a $\boldsymbol{K}$ matrix with the same eigenvalues. The initial conditions for each decay term will be different since the eigenvectors are different, but the magnitudes of the least-squares SADS are allowed to vary freely, so this difference is absorbed into the SADS as a scaling factor. The end result is that, for a given set of rates $\left\{k_{i}\right\}$ in an unbranched sequential model, permutations of the rates will give identical residuals but vastly different SADS.

In order to extract the true SADS with a compartmental model, one must impose additional constraints on the fitting based on a priori knowledge of the system [64, 73, 74]. Typically, this involves choosing a number of kinetic models to test, based on the number of apparent rates in the DAS fitting, and other information such as the structure of the system and information from other spectroscopic techniques. The rates for each model are optimized, and the model producing the most physically 'reasonable' set of SADS is chosen. This fitting approach is often called 'target analysis' in the literature, while fitting to decay associated spectra is referred to as 'global analysis' [73]. SADS can be assessed based on the known positions, signs and widths of absorptive features from constituent chromophores, 
and the order of magnitude of reasonable transfer rates can be estimated based on structural information.

\subsubsection{Temperature-Dependent Kinetic Fitting}

Another approach to constraining target analysis is by adding an additional dimension to the measured spectra. Using the temperature-dependence of transient absorption spectra has been proposed as a way to improve target analysis fitting [64, 72, 134]. Under the assumption that only the kinetic rates, and not the SADS, vary substantially over a given temperature range, the transient spectra at all temperatures can be reconstructed using only a single set of basis spectra. The model can be further constrained with the assumption that the rate

constants have an Arrhenius temperature dependence $K_{i j}(T) \propto \frac{k_{B} T}{h} e^{-\Delta E_{i j} / k_{B} T}$, where $k_{B}$ is Boltzmann's constant, $h$ is Planck's constant, and $\Delta E_{i j}$ is the activation enthalpy for each transition $[64,72]$. Then, the nonlinear parameters become $\Delta E_{i j}$, and the concentrations $\vec{c}(t, T)$ can be calculated for each temperature. The measured spectrum at each temperature is given by

$$
\mathbf{S}_{(m \times p)}(T)=\mathbf{C}_{(m \times n)}(T) \mathbf{F}_{(n \times p)}
$$

for $t=\left\{t_{1}, \ldots, t_{m}\right\}, \lambda=\left\{\lambda_{1}, \ldots, \lambda_{p}\right\}$, and $T=T_{1}, \ldots, T_{q}$. Since $\boldsymbol{F}$ is assumed to be temperatureindependent, we can reduce the $3 \mathrm{~d}$ matrix problem to the form of Equation 3.5 by 'stacking' $\boldsymbol{S}(T)$ and $\boldsymbol{C}(T)$ into matrices with $m \times q$ rows [72]: 


$$
\begin{aligned}
& {\left[\begin{array}{ccc}
{\left[\begin{array}{ccc}
S\left(\lambda_{1}, t_{1}, T_{1}\right) & \cdots & S\left(\lambda_{p}, t_{1}, T_{1}\right) \\
\vdots & \ddots & \vdots \\
S\left(\lambda_{1}, t_{m}, T_{1}\right) & \cdots & S\left(\lambda_{p}, t_{m}, T_{1}\right)
\end{array}\right]} \\
\vdots & \\
{\left[\begin{array}{ccc}
S\left(\lambda_{1}, t_{1}, T_{q}\right) & \cdots & S\left(\lambda_{p}, t_{1}, T_{q}\right) \\
\vdots & \ddots & \vdots \\
S\left(\lambda_{1}, t_{m}, T_{q}\right) & \cdots & S\left(\lambda_{p}, t_{m}, T_{q}\right)
\end{array}\right]}
\end{array}\right]=} \\
& {\left[\begin{array}{ccc}
{\left[\begin{array}{ccc}
c_{1}\left(t_{1}, T_{1}\right) & \cdots & c_{n}\left(t_{1}, T_{1}\right) \\
\vdots & \ddots & \vdots \\
c_{1}\left(t_{m}, T_{1}\right) & \cdots & c_{n}\left(t_{m}, T_{1}\right)
\end{array}\right]} \\
{\left[\begin{array}{ccc}
c_{1}\left(t_{1}, T_{q}\right) & \cdots & c_{n}\left(t_{1}, T_{q}\right) \\
\vdots & \ddots & \vdots \\
c_{1}\left(t_{m}, T_{q}\right) & \cdots & c_{n}\left(t_{m}, T_{q}\right)
\end{array}\right]}
\end{array}\right]\left[\begin{array}{ccc}
f_{1}\left(\lambda_{1}\right) & \cdots & f_{1}\left(\lambda_{p}\right) \\
\vdots & \ddots & \vdots \\
f_{n}\left(\lambda_{1}\right) & \cdots & f_{n}\left(\lambda_{p}\right)
\end{array}\right]}
\end{aligned}
$$

The SADS matrix $\boldsymbol{F}$ in Equation 3.14 can be found in the same way as before, using the pseudo-inverse of the concatenated $\boldsymbol{C}$ matrix.

\subsubsection{Excitation-Dependent Kinetic Fitting}

Another potential source of additional information is to excite the sample at different wavelengths. If the absorption bands of the chromophores are sufficiently separated, different wavelength excitation pulses can selectively excite different ratios initial states. In terms of compartmental fitting, this excitation dependence appears in the $\vec{a}(t)$ term in Equation 3.3, and depends on the overlap of the pump bandwidth with the ground state absorbance of each state.

There are several examples in the literature of transient absorption studies of BRCs $[35,51,135,136]$ and other light harvesting systems [117, 137] using multiple excitations, with various levels of complexity applied to the data analysis. In some cases, analysis was limited to a qualitative comparison of the time traces and features in the absorption spectra without global fitting $[51,135]$. Others perform a separate target analysis for each excitation wavelength, and compare features of the resulting SADS to identify common product states 
between the pathways $[35,117,137]$. This approach can in principle identify the 'true' kinetic scheme by finding a self-consistent set of models, one for each excitation, for which the SADS of common states match. However, this requires separately testing and examining countless potential models, especially when branched reactions might be present, and the criteria for 'good' agreement is qualitative. Romero et al. took this approach a step further by performing target analysis on multiple data sets simultaneously, and comparing the results to the individually fitted SADS [137].

\subsection{Global Analysis of 2DES Data}

By resolving the optical response as a function of excitation and detection frequencies, 2DES provides the same information as the narrow-band excitation TA experiments described in Section 3.1.4, but with improved population time resolution, and excitation axis resolution limited only by the pump bandwidth and $t_{1}$ sampling. Data from all excitation

conditions are collected simultaneously, under the same experimental conditions. In principle, this additional information can be used to impose new constraints for target analysis, and ideally would allow one to uniquely invert Equation 3.1 to obtain all transfer rates and SADS. The question, then, is how best to use the 2DES data to extract the population kinetics.

\subsubsection{D Analog to DADS and SADS}

A simple approach is to represent the measured 2D spectrum as a linear combination of 2D basis spectra $f_{i}\left(\lambda_{e x}, \lambda_{\text {det }}\right)$, analogously to Equation 3.1:

$$
S\left(\lambda_{e x}, \lambda_{d e t}, t\right)=\sum_{i=1}^{n} c_{i}(t) f_{i}\left(\lambda_{e x}, \lambda_{d e t}\right)
$$

where $c_{i}(t)$ is governed by the same rate constants $K_{i j}$ in Equation 3.2. Analogously to Equation 3.5, we now have a block of $2 \mathrm{D}$ data of dimension $m \times p \times q$ sampled at $m$ population times, $p$ excitation frequencies, and $q$ detection frequencies.

$$
\boldsymbol{S}_{(m \times p \times q)}=\boldsymbol{C}_{(m \times n)} \boldsymbol{F}_{(n \times p \times q)}
$$


We can reduce this to the same matrix equation as before by reshaping the $2 \mathrm{D}$ spectra at each time into a single vector with $p \times q$ columns:

$$
\boldsymbol{S}_{(m \times(p \times q))}=\boldsymbol{C}_{(m \times n)} \boldsymbol{F}_{(n \times(p \times q))}
$$

After solving Equation 3.17 as before using the pseudo-inverse of $\boldsymbol{C}$, the 2D basis spectra $f_{i}\left(\lambda_{e x}, \lambda_{\text {det }}\right)$ can be reconstructed from $\boldsymbol{F}$. (This is the method that the CarpetView (Light Conversion) analysis software uses for 2DES global fitting.)

This approach, while useful as a data visualization method, suffers the same limitations as typical global and target analysis of transient absorption spectra. By unwrapping the basis spectra into $p \times q$ points, this method treats each point $S\left(\lambda_{e x}, \lambda_{\text {det }}\right)$ as a completely independent measurement, effectively performing a separate DADS or SADS fit for each excitation wavelength. In terms of constraining the fitting, Equation 3.17 does no better than the transient absorption methods described in Section 3.1.4. The 2D SADS for a given model are not unique for the same reasons outlined in Section 3.1.2.

Two-dimensional decay associated spectra (2DDAS) are commonly used in the 2DES literature, and have been successfully applied in a number of studies on light-harvesting systems including the Fenna-Matthews-Olson complex [67], Photosystem II [68], the Fucoxanthinchlorophyll protein complex [138], and chlorophyll binding proteins [139]. Two-dimensional

evolution associated spectra (2DEAS), the 2D analogue to a compartmental SADS model, have been applied in studies of bacterial LH1 [140] and LH2 [141], and have recently been used to reveal a carotenoid dark state in purple bacteria [142]. A modified version of 2DEAS which also includes oscillatory modes, called vibration associated spectra (2DVAS), has recently been used to study coherent dynamics in 2D spectra $[141,143]$.

\subsubsection{Excitation-Dependent 2DES Fitting}

2DES measures the excitation dependence of the transient signal, but the commonly used 2DDAS and 2DEAS models do not effectively use this additional information as a constraint, requiring the user to manually check each fitting result for self-consistency and 
impose constraints accordingly. For an $n$-compartment model, 2DEAS analysis will provide $n \times p$ independent 1D basis spectra, or $n$ SADS for each of $p$ measured excitation frequencies. However, in light harvesting systems such as BRCs, excitations of different chromophores lead to common product states, which will have the same spectral signatures regardless of how the system was initially excited (except weighted by a factor dependent on the pump and probe polarizations relative to the involved transition dipoles, discussed in Section 3.3). The number of free linear parameters can be reduced if we require each excitation frequency to share SADS of common product states.

The implementation of this excitation-dependent global fitting approach is similar in form to the temperature-dependent kinetic fitting method [72] described in Section 3.1.3. For an $n$-compartment model, with $m$ time points and $p$ detection wavelengths, the signal at each excitation wavelength $\lambda_{e x}$ can be written as:

$$
\begin{aligned}
S\left(\lambda^{e x}, \lambda^{d e t}, t\right) & =\sum_{i=1}^{n} c_{i}\left(\lambda^{e x}, t\right) f_{i}\left(\lambda^{d e t}\right) \\
\mathbf{S}_{(m \times p)}\left(\lambda^{e x}\right) & =\mathbf{C}_{(m \times n)}\left(\lambda^{e x}\right) \mathbf{F}_{(n \times p)}
\end{aligned}
$$

Comparing to Equation 3.15 for the usual 2DEAS approach, Equation 3.18 moves the excitation dependence from $f_{i}$ to $c_{i}$, reflecting the fact that the spectral signatures $f_{i}$ of common product states should not depend on $\lambda_{e x}$, and the time-dependent concentrations will depend on how the sample was excited at different $\lambda_{e x}$ values. In matrix form, Equation 3.19 is analogous to Equation 3.13 from the temperature-dependent case. To simultaneously fit all $q$ excitation frequencies, we can stack $\boldsymbol{S}\left(\lambda_{e x}\right)$ and $\boldsymbol{C}\left(\lambda_{e x}\right)$ into matrices with $m \times q$ rows in the same way as Equation 3.14: 


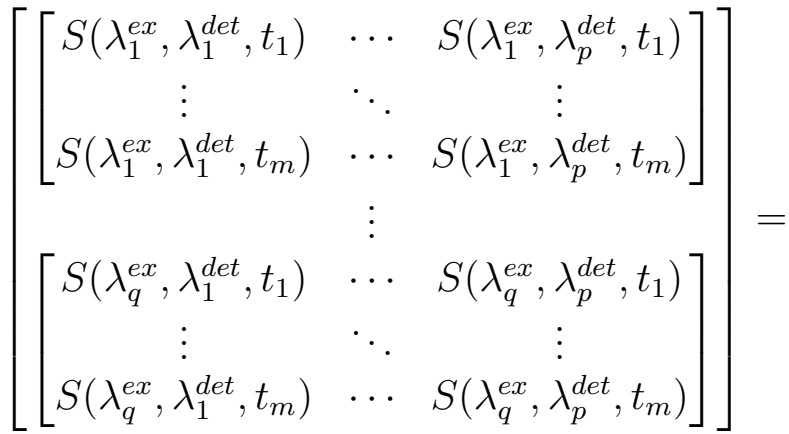

$$
\begin{aligned}
& {\left[\begin{array}{ccc}
{\left[\begin{array}{ccc}
c_{1}\left(\lambda_{1}^{e x}, t_{1}\right) & \cdots & c_{n}\left(\lambda_{1}^{e x}, t_{1}\right) \\
\vdots & \ddots & \vdots \\
c_{1}\left(\lambda_{1}^{e x}, t_{m}\right) & \cdots & c_{n}\left(\lambda_{1}^{e x}, t_{m}\right)
\end{array}\right]} \\
\vdots & \\
{\left[\begin{array}{ccc}
c_{1}\left(\lambda_{q}^{e x}, t_{1}\right) & \cdots & c_{n}\left(\lambda_{q}^{e x}, t_{1}\right) \\
\vdots & \ddots & \vdots \\
c_{1}\left(\lambda_{q}^{e x}, t_{m}\right) & \cdots & c_{n}\left(\lambda_{q}^{e x}, t_{m}\right)
\end{array}\right]}
\end{array}\right]\left[\begin{array}{ccc}
f_{1}\left(\lambda_{1}^{\text {det }}\right) & \cdots & f_{1}\left(\lambda_{p}^{\text {det }}\right) \\
\vdots & \ddots & \vdots \\
f_{n}\left(\lambda_{1}^{\text {det }}\right) & \cdots & f_{n}\left(\lambda_{p}^{\text {det }}\right)
\end{array}\right]}
\end{aligned}
$$

where the spectrum $S$ was sampled at excitation wavelengths $\lambda_{1}^{e x}, \ldots, \lambda_{q}^{e x}$, detection wavelengths $\lambda_{1}^{d e t}, \ldots, \lambda_{p}^{d e t}$, and population times $t_{1}, \ldots, t_{m}$. The pseudo-inverse of $\boldsymbol{C}$ will then give the set of $n$ least-squares optimal SADS $\left\{f_{1}\left(\lambda^{\text {det }}\right), \ldots, f_{n}\left(\lambda^{\text {det }}\right)\right\}$ which minimizes $\|\mathbf{S}-\mathbf{C F}\|_{F}$.

By making the SADS independent of $\lambda^{e x}$, there are now $n$ 1D basis spectra rather than the $n$ 2D spectra (comprised of $n \times q 1 \mathrm{D}$ spectra) we had in the 2DEAS case. However, the compartment concentration equations now require additional input to account for the excitation dependence. The initial conditions $\vec{a}(t)=\vec{a}_{0} I_{p}(t)$ from Equation 3.2 become $\vec{a}\left(\lambda^{e x}, t\right)=\vec{a}_{0}\left(\lambda^{e x}\right) I_{p}\left(\lambda^{e x}, t\right)$, and the new concentration equations are then:

$$
\frac{d}{d t} c_{i}\left(\lambda^{e x}, t\right)=\sum_{j=1}^{n} K_{i j} c_{j}\left(\lambda^{e x}, t\right)+a_{i}\left(\lambda^{e x}, t\right)
$$

with the same analytic solution as before:

$$
\vec{c}\left(\lambda^{e x}, t\right)=e^{K t} * \vec{a}\left(\lambda^{e x}, t\right)
$$

The constants $\vec{a}_{0}\left(\lambda^{e x}\right)$ are the relative contributions of the absorption spectra $\sigma_{i}(\lambda)$ of each species to the total linear absorption spectrum, such that $\sigma(\lambda)=\sum_{i}^{n} \sigma_{i}(\lambda)$. Each element $a_{i}\left(\lambda^{e x}\right)$ is then the probability of excitation of that species by the pump. For the optical 
density $\sigma(\lambda)$, the absorption probability of a given photon is $P_{a b s}(\lambda)=1-10^{-\sigma(\lambda)}$, so

$$
a_{i}\left(\lambda^{e x}\right)=\left(1-10^{-\sigma_{i}\left(\lambda^{e x}\right)}\right) I_{p}\left(\lambda^{e x}\right)
$$

where $I_{p}$ is proportional to the pump photon flux at the sample, integrated over the wavelength bin defined as $\lambda^{e x}$.

Note that, since the fitting relies on accurate relative concentrations between different excitation bins due to sharing the same SADS, it is important that the measured pump spectrum is accurate. The wavelength dependence of the instrument response, including the diffraction efficiency of the spectrometer grating, the CCD quantum efficiency, and the reflectance of all optics between the sample and spectrometer can significantly affect the initial conditions for the fitting.

The initial conditions $\vec{a}_{0}\left(\lambda^{e x}\right)$ can be calculated by fitting $\sigma(\lambda)=\sum_{i}^{n} \sigma_{i}(\lambda)$, where the peak positions, widths, and dipole strengths of each lineshape $\sigma_{i}(\lambda)$ are unknown. This fitting can either be performed (i) once, before the 2DES nonlinear optimization, or (ii) concurrently with the 2DES optimization. Each option presents different challenges.

(i) Performing a single optimization of the linear absorption spectrum before the 2DES fitting is simpler to implement, but less constrained. The peak shapes (i.e. Gaussian, Lorentzian, Voigt), positions, widths, and dipole moments can either be treated as free parameters, or fixed based on a priori knowledge of the system. The initial concentrations $\vec{a}_{0}\left(\lambda^{e x}\right)$ are computed once, using Equation 3.23, and the only nonlinear variables in the 2DES optimization are the rate constants $K_{i j}$. The advantage of this approach is that, with fewer nonlinear parameters, the 2DES fitting converges faster, and is less prone to local minima. The disadvantage is that the $\operatorname{SADS} f_{i}(\lambda)$ and the linear spectra $\sigma_{i}(\lambda)$ are treated completely independently. The GSB and SE signals from each initially excited state should have peak positions and widths corresponding to the linear absorption spectrum. The agreement between $f_{I}$ and $\sigma_{i}$ can be used as a criterion for assessing the validity of a given model. 
(ii) The second option includes the peak positions, widths, and dipole strengths as nonlinear parameters in the 2DES fitting. The residual supplied to the nonlinear optimizer is a weighted sum of the 2DES residual $\|\mathbf{S}-\mathbf{C F}\|_{F}$ and the linear absorption residual $\sigma(\lambda)-\sum_{i}^{n} \sigma_{i}(\lambda)$. The choice of weighting factor drastically affects the fitting results, and the nonlinear optimization can produce nonphysical results if one residual is disproportionately favored over the other. By favoring the 2DES residual, the optimizer is essentially free to choose any initial conditions for the compartments. By favoring the linear absorption residual, the optimization will prioritize the lineshape parameters first, then find the best rate constants, which essentially produces the same result as (i).

A similar 2DES kinetic fitting approach to the one outlined in this section was recently described by Dostal et al [75], and applied to the study of energy transfer in the FMO complex [67]. Their method uses the typical linear kinetic model for the populations, and includes excitation frequency dependence in terms of the line shapes and transition dipoles of the initially excited species in the same way as described above. The primary difference is that they constrain the SADS to have the same line shapes as the ground state spectra used for the initial conditions. The $2 \mathrm{D}$ spectrum is then reconstructed as the matrix product [75]:

$$
\boldsymbol{S}_{\left(n_{3} \times n_{1}\right)}^{2 D}(t)=\boldsymbol{L}_{3\left(n_{3} \times N\right)} \boldsymbol{M}_{(N \times N)} \boldsymbol{F}_{(N \times N)} \exp \left(\boldsymbol{K}_{(N \times N)} t\right) \boldsymbol{M}_{(N \times N)} \boldsymbol{L}_{1\left(N \times n_{1}\right)}
$$

where $\boldsymbol{K}$ is the same rate constant matrix as before, $t$ is population time, $\boldsymbol{M}$ is a diagonal matrix with transition dipole squares $\mu_{i}^{2}, \boldsymbol{L}_{1}$ and $\boldsymbol{L}_{3}$ are line shape functions sampled at the excitation and detection frequencies, respectively, and $\boldsymbol{F}$ defines the amplitudes of contributions from each $\boldsymbol{L}_{3}$ line shape to the transient signal of each species. They perform a global nonlinear optimization with respect to the rate constants $\boldsymbol{K}$, dipole strengths $\mu_{i}^{2}$, linear absorption peak positions and widths of each species. The $N^{2}$ elements of $\boldsymbol{F}$ are linear parameters.

In terms of the fitting approach described by Equations 3.19-3.23, $\boldsymbol{M} \boldsymbol{L}_{\mathbf{1}}$ are the initial conditions $\vec{a}\left(\lambda^{e x}\right)$ in the impulsive limit where $\vec{a}(t)=\vec{a}_{0} \delta(t)$. So, $\exp (\boldsymbol{K} t) \boldsymbol{M} \boldsymbol{L}_{\mathbf{1}}$ gives the time-dependent concentrations in Equation 3.22. The product $\boldsymbol{L}_{3} \boldsymbol{M} \boldsymbol{F}$ in Equation 3.24 is 
analogous to the SADS matrix $\boldsymbol{F}$ from Equation 3.19.

The main difference between the two approaches is in how the SADS are calculated. The first approach computes the SADS as a linear least squares problem, leaving only the rate constants as nonlinear parameters in the 2DES fitting. The resulting SADS are analogous to transient absorption fitting results, but constrained based on excitation conditions. The second method (Equation 3.24) constructs the SADS from the nonlinear fitting parameters (i.e. peak positions, widths, dipole moments) and solves for $\boldsymbol{F}$ by linear least squares. This constrains the resulting SADS to a form that is consistent with the model assumptions.

Equation 3.24 does not reproduce "off-grid" ESA signals, or ESA at detection frequencies which do not correspond to any of the $N$-species absorption peaks [75], whereas the least-squares approach imposes no such constraints. SADS associated with charge transfer states, such as the $\mathrm{P}^{+} \mathrm{H}_{A}^{-}$state in BRCs, exhibit electrochromic shifts which are not well-represented by combinations of the linear absorption bands assumed in Equation 3.24. Simultaneous kinetic fitting with other types of time-resolved data, such as 2D Electronic Stark Spectroscopy (2DESS) spectra [144] also requires a different set of basis spectra than combinations of linear spectra.

It is possible to combine the benefits of both approaches by constraining some of the basis spectra using information from the linear absorption spectrum, while allowing a the rest to be unconstrained linear least squares solutions. If we partition the basis spectra $\boldsymbol{F}$ from Equation 3.20 into unconstrained and constrained terms, we can write $\boldsymbol{C}$ and $\boldsymbol{F}$ as block matrices:

$$
\boldsymbol{S}=\left[\begin{array}{ll}
\boldsymbol{C}_{1} & \boldsymbol{C}_{2}
\end{array}\right]\left[\begin{array}{l}
\boldsymbol{F}_{1} \\
\boldsymbol{F}_{2}
\end{array}\right]
$$

where $\boldsymbol{F}_{1}$ is $n_{1} \times p, \boldsymbol{F}_{2}$ is $n_{2} \times p$, and $N=n_{1}+n_{2}$. The concentration matrices $\boldsymbol{C}_{1}$ and $\boldsymbol{C}_{2}$ are constructed from the columns of $\boldsymbol{C}$ associated with the constrained and unconstrained spectra. Let $\boldsymbol{F}_{1}=\boldsymbol{G} \boldsymbol{L}$, where $\boldsymbol{G}$ is $n_{1} \times n_{1}$ and the rows of $\boldsymbol{L}$ are linear absorption spectra 
analogous to $\boldsymbol{M} \boldsymbol{L}_{\mathbf{1}}$ in Equation 3.24. Taking the pseudo-inverse of 3.25,

$$
\left[\begin{array}{ll}
\boldsymbol{C}_{1} & \boldsymbol{C}_{2}
\end{array}\right]^{+} \boldsymbol{S}=\left[\begin{array}{c}
\boldsymbol{S}_{1}^{\prime} \\
\boldsymbol{S}_{2}^{\prime}
\end{array}\right]=\left[\begin{array}{c}
\boldsymbol{G L} \\
\boldsymbol{F}_{2}
\end{array}\right]
$$

The coefficients $\boldsymbol{G}$ for the constrained basis spectra can be found from $\boldsymbol{G} \boldsymbol{L}=\boldsymbol{S}_{1}^{\prime}$, and the unconstrained least-squares spectra $\boldsymbol{F}_{2}=\boldsymbol{S}_{2}^{\prime}$. The input spectra $\boldsymbol{L}$ do not necessarily have to be in the form of Gaussian peaks from the linear absorption spectrum that Dostal et al. assume. If, for instance, we know the form of a subset of the basis spectra based on a priori knowledge, then $\boldsymbol{G}$ becomes a diagonal matrix of scaling factors for each row of $\boldsymbol{L}$.

\subsubsection{Simultaneous Fitting of Multiple Data Sets}

The 2DES kinetic fitting approach described in Section 3.2.2 can be extended to simultaneously fit multiple data sets with different types of signals which depend on the same kinetic rates $\boldsymbol{K}$. These data sets can potentially be sampled over different frequency ranges and population times. This may prove useful for finding a kinetic model which simultaneously fits 2DES and 2DESS or TGESS [144] data, or for combining 2DES scans with different detection axis ranges.

If all data sets involved in the fitting are modeled by an $n$-compartment kinetic scheme with the same rate constants, then Equation 3.22 gives the concentrations $\vec{c}\left(\lambda^{e x}, t\right)$ for each data set. Then each set can be arranged in the form of Equation 3.20, and the residual supplied to the optimizer is the sum of residuals $\|\mathbf{S}-\mathbf{C F}\|_{F}$ from each set. If the excitation and time axes are the same for all data sets, then only one pseudo-inverse $\boldsymbol{C}^{+}$must be computed for each iteration.

Another potential extension of the fitting method in Section 3.2.2 is to temperaturedependent 2DES data. The 2D analogue to the temperature-dependent transient absorption fitting described in Section 3.1.3 would involve computing the concentrations as a function of both excitation frequency and temperature. Assuming, for instance, an Eyring or Arrhenius temperature dependence for the rate constants [73], we can compute a concentration matrix $\boldsymbol{C}$ in Equation 3.20, with $m \times q$ rows and $n$ columns, for each temperature. Then, if the SADS 
are temperature-independent (which may not be a good assumption if changes in thermal broadening are significant over the temperature range), the blocks of data $\boldsymbol{S}_{((m \times q) \times p)}(T)$ and concentrations $\boldsymbol{C}_{((m \times q) \times n)}(T)$ can be stacked into a matrices with $m \times q \times r$ rows, and we get a single, larger linear least squares problem:

$$
\left[\begin{array}{c}
\boldsymbol{S}_{((m \times q) \times p)}\left(T_{1}\right) \\
\vdots \\
\boldsymbol{S}_{((m \times q) \times p)}\left(T_{r}\right)
\end{array}\right]=\left[\begin{array}{c}
\boldsymbol{C}_{((m \times q) \times n)}\left(T_{1}\right) \\
\vdots \\
\boldsymbol{C}_{((m \times q) \times n)}\left(T_{r}\right)
\end{array}\right]\left[\begin{array}{ccc}
f_{1}\left(\lambda_{1}^{\text {det }}\right) & \cdots & f_{1}\left(\lambda_{p}^{\text {det }}\right) \\
\vdots & \ddots & \vdots \\
f_{n}\left(\lambda_{1}^{\text {det }}\right) & \cdots & f_{n}\left(\lambda_{p}^{\text {det }}\right)
\end{array}\right]
$$

where the temperature is sampled at $T_{1}, \ldots, T_{r}$.

\subsection{The Magic Angle and Polarization Associated Spectra}

The probability of a transition from an initial state $i$ to state $f$ in a molecular system depends on the orientation of the molecule relative to the polarization of the light source. In the limit where an electric field interaction with the molecule can be treated perturbatively to first-order, the transition probability $P_{f i} \propto\left|\boldsymbol{\mu}_{f i} \cdot \boldsymbol{E}\right|^{2}$, where $\boldsymbol{\mu}_{f i}$ is the transition dipole moment (TDM) and $\boldsymbol{E}$ is the electric field vector [87, 145]. For a transient absorption or 2DES experiment, the transition probabilities for a given interaction with the pump pulse and the probe pulse are weighted by the orientation factors [145]:

$$
\begin{aligned}
& P_{p u}=\left|\boldsymbol{\mu}_{p u} \cdot \boldsymbol{E}_{p u}\right|^{2} \\
& P_{p r}=\left|\boldsymbol{\mu}_{p r} \cdot \boldsymbol{E}_{p r}\right|^{2}
\end{aligned}
$$

where $\boldsymbol{\mu}_{p u}$ and $\boldsymbol{\mu}_{p r}$ are TDMs of arbitrary transitions induced by the pump and probe, respectively. The total detected signal is the sum of signal contributions from each randomly oriented sample. This complicates the interpretation of time-resolved spectra, where the initially excited TDM is not generally aligned with TDMs of the product states.

For any given molecule, the probability of detecting a signal corresponding to excitation of state $i$ with pumped TDM $\boldsymbol{\mu}_{i}$, and detection of state $f$ with probed TDM $\boldsymbol{\mu}_{f}$ will be proportional to an orientation-dependent factor:

$$
\hat{P}_{i j}=\left|\hat{\mu}_{i} \cdot \hat{e}_{p u}\right|^{2}\left|\hat{\mu}_{j} \cdot \hat{e}_{p r}\right|^{2}
$$


where $\hat{\mu}$ and $\hat{e}$ are TDP and electric field unit vectors, respectively. The total detected signal corresponding to that transition is the rotational average of Equation 3.30 for all possible orientations of the molecule with respect to the pump and probe. Schott et al. perform this rotational averaging analytically for arbitrary pump and probe polarizations (including elliptical polarizations), pump/probe crossing angle, and angle $\alpha_{i j}$ between $\hat{\mu}_{i}$ and $\hat{\mu}_{j}$ [145].

In our case, pump and probe polarizations are always linear, and we get an orientation factor which depends only on $\alpha$ and the angle $\phi$ between $\hat{e}_{p u}$ and $\hat{e}_{p r}$ :

$$
\hat{P}_{i j}\left(\alpha_{i j}, \phi\right)=\frac{1}{60}\left[7+\cos (2 \phi)+\cos \left(2 \alpha_{i j}\right)(1+3 \cos (2 \phi))\right]
$$

The total signal is the sum of signal contributions from all populated states at time $t$ after excitation. If we excite state $i$, which then transfers population to product states $j=1, \ldots, n$, the signal contribution from that excitation is:

$$
S_{i}\left(\lambda^{\text {det }}, t\right)=\sum_{j=1}^{n} c_{i j}(t) f_{j}\left(\lambda^{\text {det }}\right) \hat{P}\left(\alpha_{i j}, \phi\right)
$$

where $c_{i j}(t)$ are the concentrations of each state $j$ after initial excitation of only state $i$, and $f_{j}\left(\lambda^{d e t}\right)$ are the difference spectra for each state. In terms of the kinetic fitting in Section $3.2 .2, c_{i j}(t)$ would be the solution to Equation 3.22 with $\vec{a}=\delta_{i j} I_{p}(t)$ initially populating only state $i$.

If there are overlapping ground state absorption spectra for different species, then excitation at a given frequency will populate several states, and the signal will be a sum of contributions $S_{i}$ from each excitation:

$$
S\left(\lambda_{e x}, \lambda_{d e t}, t\right)=\sum_{i} S_{i}\left(\lambda_{d e t}, t\right)=\sum_{i=1}^{n} \sum_{j=1}^{n} c_{i j}(t) f_{j}\left(\lambda_{d e t}\right) \hat{P}\left(\alpha_{i j}, \phi\right)
$$

The total signal cannot be written as $\sum_{i} c_{i}\left(\lambda_{e x}, t\right) f_{i}\left(\lambda_{\text {det }}\right)$ as in Equation 3.18, because the signal from a molecule in state $j$ depends on which state $i$ was initially excited. The excitationdependent 2D fitting approach described in Section 3.2.2 works under the assumption that the basis spectra $f_{j}\left(\lambda_{\text {det }}\right)$ are only weighted by their time-dependent concentrations. If the transition dipoles of the excited states are unknown, then the dipole terms $\alpha_{i j}$ add additional 
free parameters to the model.

Equation 3.33 reduces to the simpler, orientation-independent form of Equation 3.18 that we assumed for the linear kinetic model if $\hat{P}(\alpha, \phi)$ is constant. Examining Equation 3.31, the only $\alpha$-dependent term is multiplied by $(1+3 \cos (2 \phi))$. Setting this term equal to zero yields $\phi_{\mathrm{MA}}=\cos ^{-1}(1 / \sqrt{3}) \approx 54.7^{\circ}$. This is known as the "Magic Angle" (MA), and widely used transient absorption spectroscopy $[17,49,106,108,111,117,146]$ with an analogous condition for 2D spectroscopy [67, 147, 148].

Evaluating Equation 3.31 at $\phi=\{0, \pi\}$ corresponding to probe polarizations parallel and perpendicular to the pump, respectively, we get signals $S_{\|}$and $S_{\perp}$ weighted by the following dipole orientation factors:

$$
\begin{aligned}
S_{\|} & \propto \frac{1}{15}\left(2+\cos \left(2 \alpha_{i j}\right)\right) \\
S_{\perp} & \propto \frac{1}{30}\left(3-\cos \left(2 \alpha_{i j}\right)\right)
\end{aligned}
$$

The dependence on $\alpha_{i j}$ cancels if we take $S_{\|}+2 S_{\perp}$ :

$$
\begin{aligned}
\frac{1}{3}\left(S_{\|}\left(\alpha_{i j}\right)+2 S_{\perp}\left(\alpha_{i j}\right)\right) & =\hat{P}_{i j}\left(\alpha_{i j}, \phi_{\mathrm{MA}}\right) \\
& =S_{\mathrm{MA}}\left(\alpha_{i j}\right)=\text { const. }
\end{aligned}
$$

So, the magic angle signal contribution $S_{\mathrm{MA}}$ from a given transition with $\alpha_{i j}$ is a linear combination of the $S_{\|}\left(\alpha_{i j}\right)$ and $S_{\perp}\left(\alpha_{i j}\right)$ signals. From Equation 3.33, we have that the total measured signal measured with polarization $\phi$ is the sum of signal contributions from each transition, with different dipole angles $\alpha_{i j}$. It is straightforward to show that combining the measured 2DES signals $S_{\|}\left(\lambda_{e x}, \lambda_{\text {det }}, t\right)$ and $S_{\perp}\left(\lambda_{e x}, \lambda_{\text {det }}, t\right)$ yields the $\alpha$-independent magic angle 2D spectrum: 


$$
\begin{aligned}
S_{\|}\left(\lambda_{e x}, \lambda_{\text {det }}, t\right)+2 S_{\perp}\left(\lambda_{\text {ex }}, \lambda_{\text {det }}, t\right) & =\sum_{i=1}^{n} \sum_{j=1}^{n} c_{i j}(t) f_{j}\left(\lambda_{\text {det }}\right)\left(\hat{P}\left(\alpha_{i j}, 0\right)+2 \hat{P}\left(\alpha_{i j}, \pi / 2\right)\right) \\
& =\sum_{i=1}^{n} \sum_{j=1}^{n} c_{i j}(t) f_{j}\left(\lambda_{\text {det }}\right)\left(3 \hat{P}\left(\alpha_{i j}, \phi_{\mathrm{MA}}\right)\right) \\
& =3 S_{\mathrm{MA}}\left(\lambda_{\text {ex }}, \lambda_{\text {det }}, t\right)
\end{aligned}
$$

Therefore, to obtain an anisotropy-independent 2D spectrum suitable for kinetic fitting with the method in Section 3.2.2, one can either directly measure the magic angle spectrum, or reconstruct it from 2D spectra with parallel and perpendicular polarizations.

The parallel and perpendicular polarization components can also be combined to generate polarization associated spectra (PAS) defined as [67, 149]:

$$
\begin{aligned}
& S_{z}=\frac{1}{3}\left(S_{\|}+2 S_{\perp}\right)\left(5 \frac{S_{\|}-S_{\perp}}{S_{\|}+2 S_{\perp}}+1\right) \\
& S_{y}=\frac{1}{3}\left(S_{\|}+2 S_{\perp}\right)\left(2-5 \frac{S_{\|}-S_{\perp}}{S_{\|}+2 S_{\perp}}\right)
\end{aligned}
$$

Substituting Equations 3.34 and 3.35 for $S_{\|}$and $S_{\perp}$, these expressions reduce to

$$
\begin{aligned}
& S_{z}=\cos ^{2}\left(\alpha_{i j}\right) / 3 \\
& S_{y}=\sin ^{2}\left(\alpha_{i j}\right) / 3
\end{aligned}
$$

Therefore, the $S_{z}$ PAS component enhances the signal components associated with pumped and probed states with parallel transition dipoles, while $S_{y}$ enhances those terms with orthogonal transition dipoles. The $S_{y}$ spectrum can be useful for isolating signatures of product states that are obscured by the large stimulated emission signals on the diagonal of 2D spectra [67].

Figure 3.1 shows the $\alpha$-dependence of the terms defined in Equations 3.34-3.39. The $S_{\|}$ term is maximized when the TDMs are aligned and minimized when they are perpendicular, but note that there is a nonzero $S_{\|}$signal from perpendicular transition dipoles. This is because the rotational average includes orientations where the parallel pump and probe 
polarizations have nonzero projections onto $\hat{\mu}_{i}$ and $\hat{\mu}_{j}$. Likewise, $S_{\perp}$ has a nonzero signal from parallel TDMs. The $S_{z}$ and $S_{y}$ components reveal similar information as $S_{\|}$and $S_{\perp}$, but with greater contrast. Also note that the sum of $S_{z}$ and $S_{y}$ also gives the $\alpha$-independent magic angle spectrum.

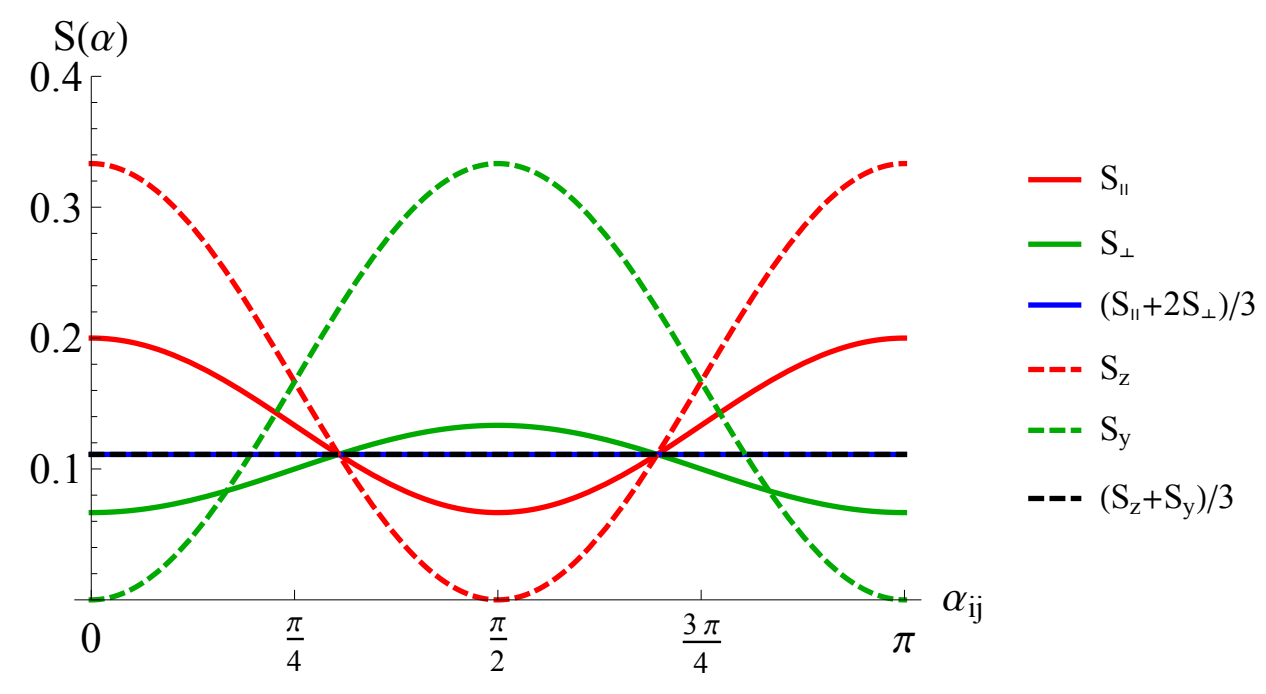

Figure 3.1: Dipole orientation dependence of the parallel $\left(S_{\|}\right)$, perpendicular $\left(S_{\perp}\right), S_{z}$, and $S_{y}$ signals as a function of angle $\alpha_{i j}$ between state transition dipoles. 


\section{CHAPTER 4}

\section{DES KINETIC FITTING OF BRC MUTANTS}

The kinetics of energy transfer and charge separation in BRCs has been studied extensively using transient absorption and time-resolved fluorescence experiments, under a variety of excitation conditions. Many studies perform some form of global analysis [22, 49, 107, $110,132]$ and/or target analysis $[35,48,49,53,107,136,150,151]$, with the general approach being to do a separate parallel or sequential fit for each excitation frequency. Comparing the resulting SADS from sequential models in different studies, the product states share similar features, especially the $\mathrm{P}^{+} \mathrm{H}_{A}^{-}$state, but the resulting time constants vary significantly.

Differences in measured SADS components and time constants can potentially be attributed to variation in experimental conditions, such as the choice of solvent, glycerol content, and pump fluence. Structural differences between BRC strains and point mutations can also affect spectral signatures and kinetic rates. However, another likely explanation for the wide variation in fitting results reported in the literature is that, as discussed in Section 3.1.2, linear kinetic models using a single excitation condition are not unique. For instance, modeling the often-cited charge separation pathway $\mathrm{P}^{*} \rightarrow \mathrm{P}^{+} \mathrm{B}_{A}^{-} \rightarrow \mathrm{P}^{+} \mathrm{H}_{A}^{-}$, one can obtain an equally "good" fit in the least-squares sense by assuming a fast component followed by a slow component, or vice versa. Both optimized models result in identical residuals, but different SADS. Furthermore, the nonlinear optimization problem with respect to rate constants is often poorly conditioned, so that large changes in the rate constants result in negligible differences in the residual. 
The ultimate goal in performing a 2DES target analysis is to test various proposed charge transfer pathways against a more stringent set of constraints than is possible with singleexcitation transient absorption experiments. Dostal et al. [75] have shown that, under ideal circumstances where the ground state absorption bands are sufficiently separated, and in the absence of dark states and inhomogeneity, the 2DES spectrum can be uniquely inverted to find the rates and associated spectra. However, despite having well-separated P,B and $\mathrm{H}$ absorption bands in the $\mathrm{BRC}$, the overlap of $\mathrm{B}_{A}$ with $\mathrm{B}_{B}$, and $\mathrm{H}_{A}$ with $\mathrm{H}_{B}$, makes it challenging to disentangle the signal contributions from the active A-branch and inactive B-branch energy transfer. Also, the degree of inhomogeneity in peak positions and rate constants is difficult to determine with a linear kinetic model, and may vary between mutants.

The overlapping A- and B-branch spectra, along with the similar energy transfer rates in both branches, result in many of the same challenges for 2DES fitting that are present in TA kinetic fitting. The 2DES residual often contains local minima with nearly degenerate rate constants and linearly dependent basis spectra which result in non-physical solutions. Other non-physical solutions arise when the model is given more compartments than are resolvable with the given data set, in which case the least-squares SADS for low-population compartments can have amplitudes orders of magnitude larger than those of the other compartments. Given these challenges, it is important to ensure that a given 2DES kinetic fit (i) makes physical sense in terms of the features present in the SADS and the order of magnitude of the resulting rates, and (ii) is not equivalent to a different kinetic model that fits the data equally well.

Chapter 4 is organized as follows. In Section 4.1, a representative subset of the measured 2DES spectra is presented and discussed. Section 4.2 shows the results from the commonly used 2DDAS and 2DEAS fitting approaches described in Section 3.2.1. We discuss what information can be gleaned from these types of decompositions, and their limitations in quantitatively distinguishing between different reaction schemes. Section 4.3 examines subsets of the measured 2DES spectra at specific excitation frequencies, which correspond to 
transient absorption spectra excited by a narrow-bandwidth pump. The apparent rates and basis spectra from individual excitation bands are carefully compared to results from published excitation-dependent transient absorption experiments. Section 4.4 presents the results of the excitation-dependent 2DES fitting technique described in Section 3.2.2, which tests the information contained in the entire 2D data set against the kinetic models proposed in the literature and discussed in Section 1.2.

\subsection{Real Absorptive 2DES Data}

Before performing any fitting, we can make a number of qualitative observations from the structure and apparent timescales depicted in the 2D spectra, which will guide our choice of potential kinetic models to test. In this section, we present 2DES with magic angle polarization, as well as the polarization associated spectra, from both W(M250)V and DLL mutant BRCs, and comment on the spectral features directly observable from the data.

\subsubsection{The W(M250)V Mutant}

The real absorptive 2DES spectra from W(M250)V are shown in Figure 4.2 for several representative population times. At early population times, the cross-section of the 2D spectrum along the diagonal is predominantly SE and GSB components from the initial excitation, with features corresponding to the linear absorption spectrum. There are clearly discernible diagonal peaks corresponding to the $\mathrm{P}, \mathrm{B}$, and $\mathrm{H}$ bands. The splitting of the absorption peaks of the $\mathrm{B}_{A}$ and $\mathrm{B}_{B}$ pigments is apparent from the broadening of the B-band peak along the diagonal. This B-band splitting is also visible as a shoulder on the red side of the B-band in the $77 \mathrm{~K}$ linear absorption spectrum of Figure 4.1.

Within the first 30fs, there is already significant energy transfer from $\mathrm{H} \rightarrow \mathrm{B}$, and from $\mathrm{B} \rightarrow \mathrm{P}$, indicated by the $\mathrm{H} / \mathrm{B}$ and $\mathrm{B} / \mathrm{P}$ cross-peaks below the diagonal. The spectrum along the detection axis at $11500 \mathrm{~cm}^{-1}$ excitation frequency corresponds to excitation of $\mathrm{P}^{*}$, and shows a weak ESA signal at $12400 \mathrm{~cm}^{-1}$. By 250fs, an H/P cross-peak appears, which indicates $\mathrm{H} \rightarrow \mathrm{P}$ energy transfer occurs on a $100 \mathrm{fs}$ timescale, likely through a $\mathrm{B}^{*}$ intermediate 


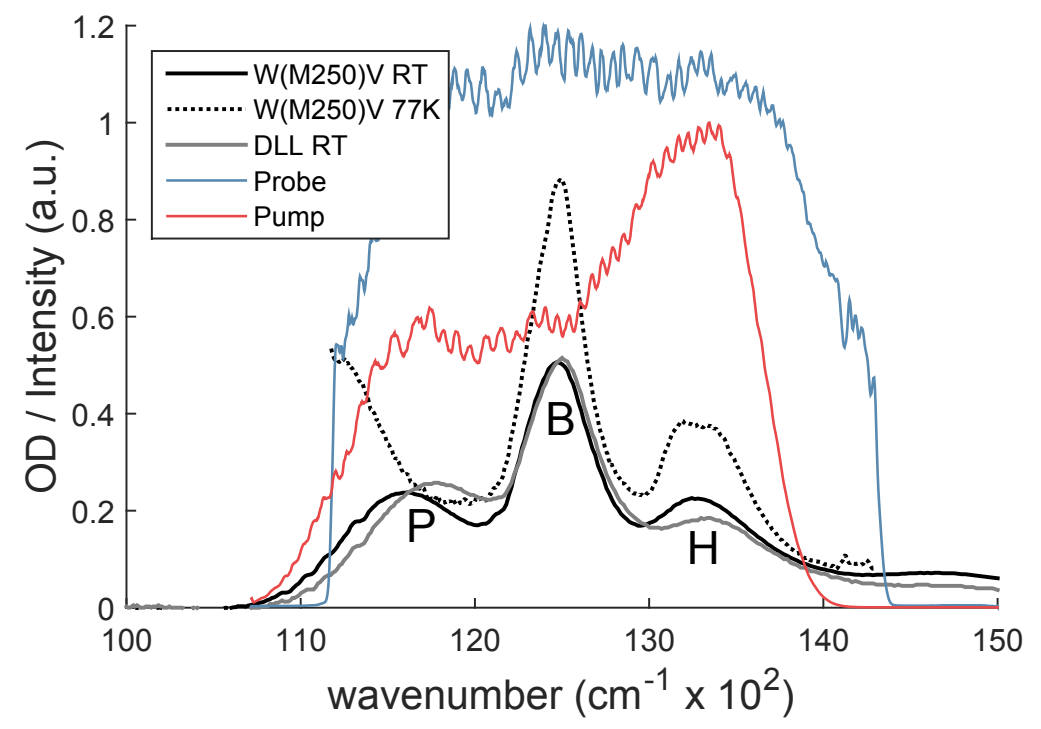

Figure 4.1: Linear absorption spectra from W(M250)V and DLL vs wavenumber

[16]. The diagonal B-band peak shows inhomogeneous broadening corresponding to $\mathrm{B}_{A}$ and $\mathrm{B}_{B}$ excitations.

At 500fs, the H-band diagonal peak has decayed almost entirely, and a second $\mathrm{H} / \mathrm{B}$ crosspeak appears at $12300 \mathrm{~cm}^{-1}$ corresponding to energy transfer from $\mathrm{H}$ to the lower-energy $\mathrm{B}_{B}$ pigment. We should be able to resolve from this splitting the $\mathrm{H} \rightarrow \mathrm{B}$ energy transfer rates on each branch. The B and P-excitation bands show similar broad, negative ESA features above the diagonal, indicating the formation of a common product state.

Between 500fs and 1.5ps, we observe a growth in the amplitude of the negative ESA peaks present at $11500 \mathrm{~cm}^{-1}$ and $12400 \mathrm{~cm}^{-1}$ excitation frequencies, as well as a pronounced splitting of the B-band peaks near the diagonal, evolving from the inhomogeneously broadened diagonal peak at early times to two horizontal bands at detection frequencies $12270 \mathrm{~cm}^{-1}$ and $12500 \mathrm{~cm}^{-1}$. The cross-section of the $1.5 \mathrm{ps} 2 \mathrm{D}$ spectra with B-band excitation frequency resembles the $\mathrm{P}^{+} \mathrm{B}_{A}^{-}$difference spectrum reported in a number of TA studies $[35,49,107]$, which is consistent with the initiation of charge separation on a picosecond timescale. After the first several picoseconds, the 2D spectrum exhibits little change; the B-band splitting vanishes, and the detected spectra from $\mathrm{P}, \mathrm{B}$, and $\mathrm{H}$ excitations all resemble the same prod- 

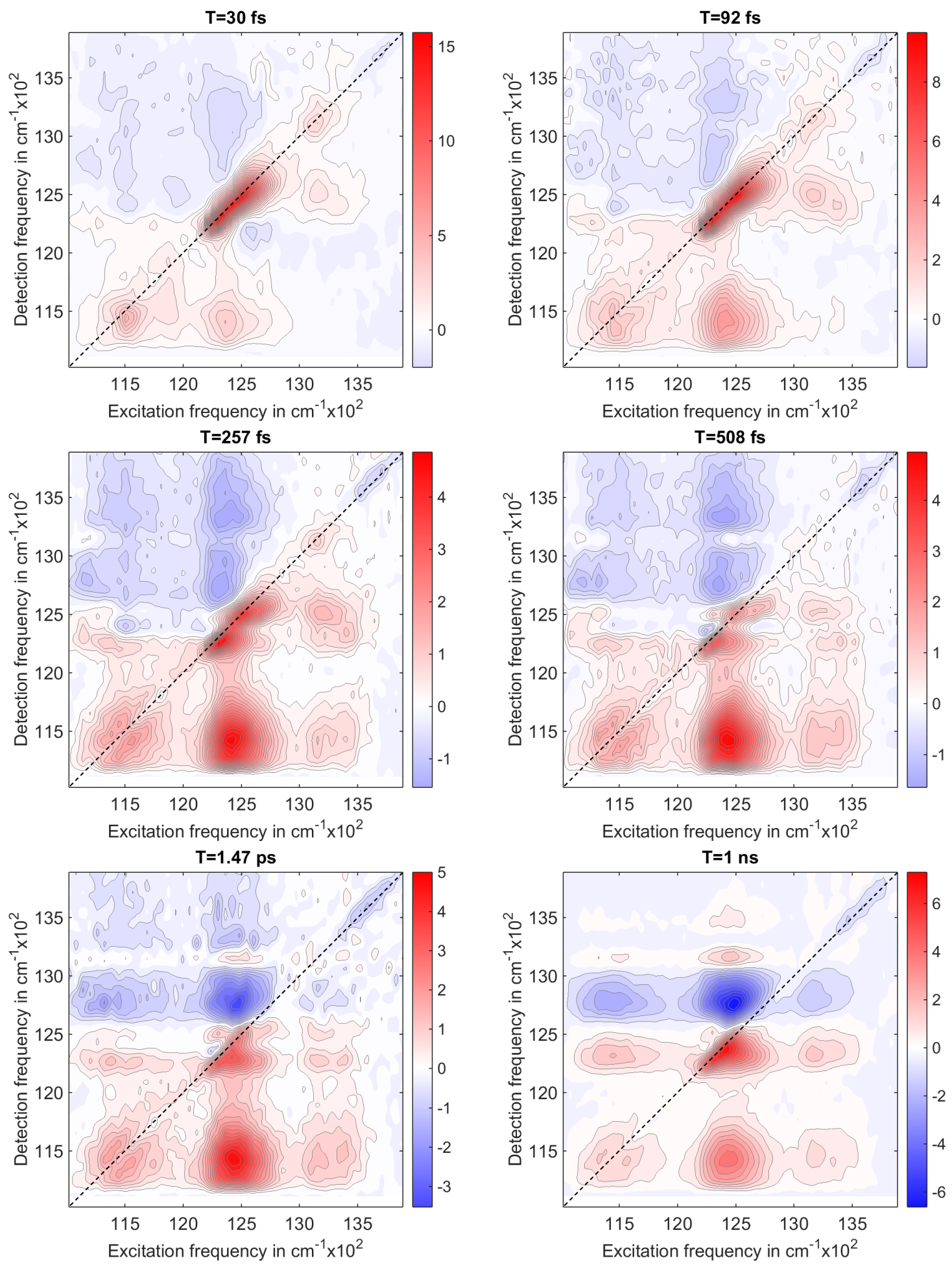

Figure 4.2: Absorptive 77K 2DES spectra from W(M250)V mutant BRCs with magic angle polarization 

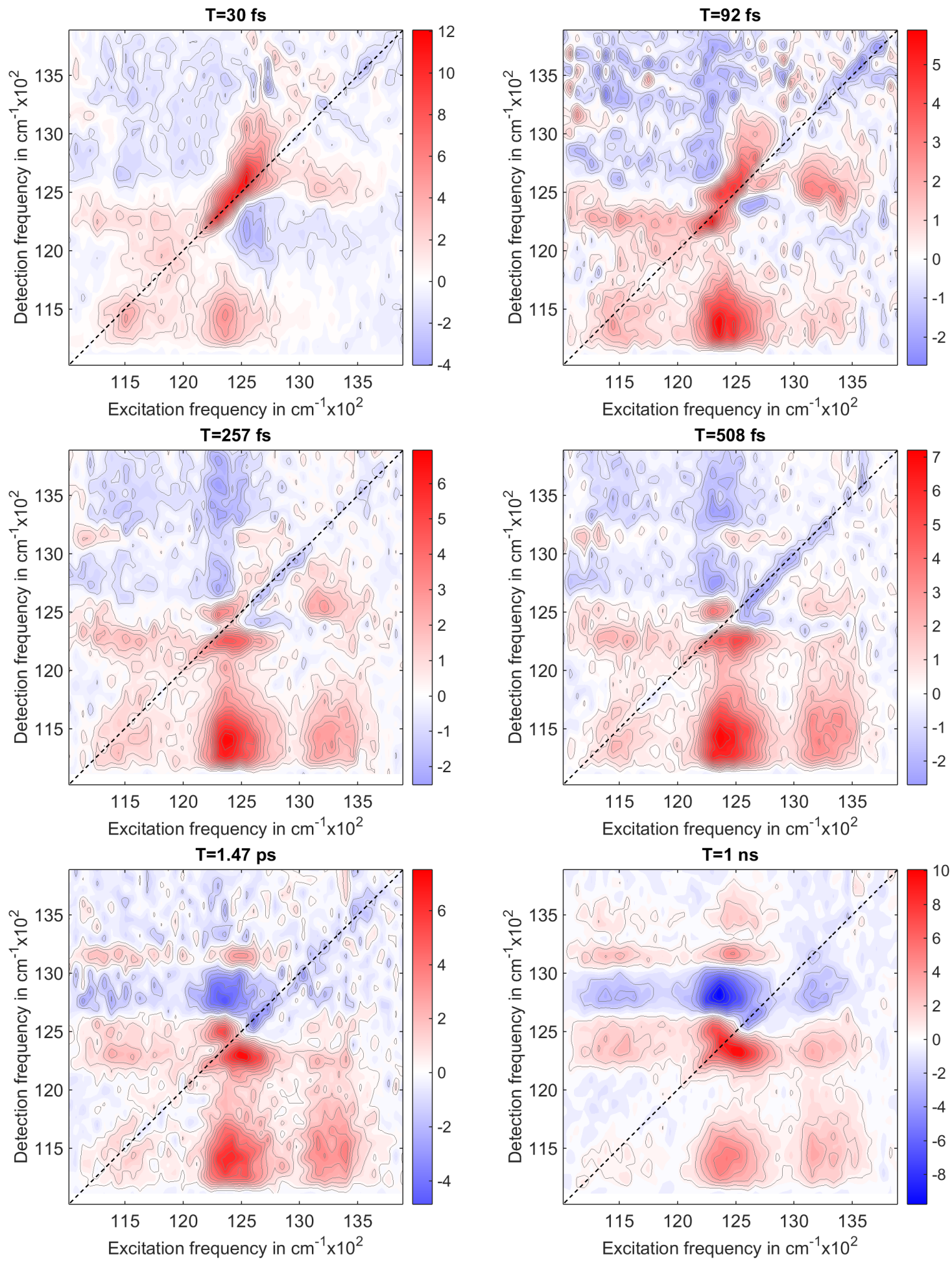

Figure 4.3: $S_{y}$ polarization associated spectra from $\mathrm{W}(\mathrm{M} 250) \mathrm{V}$ at $77 \mathrm{~K}$ 

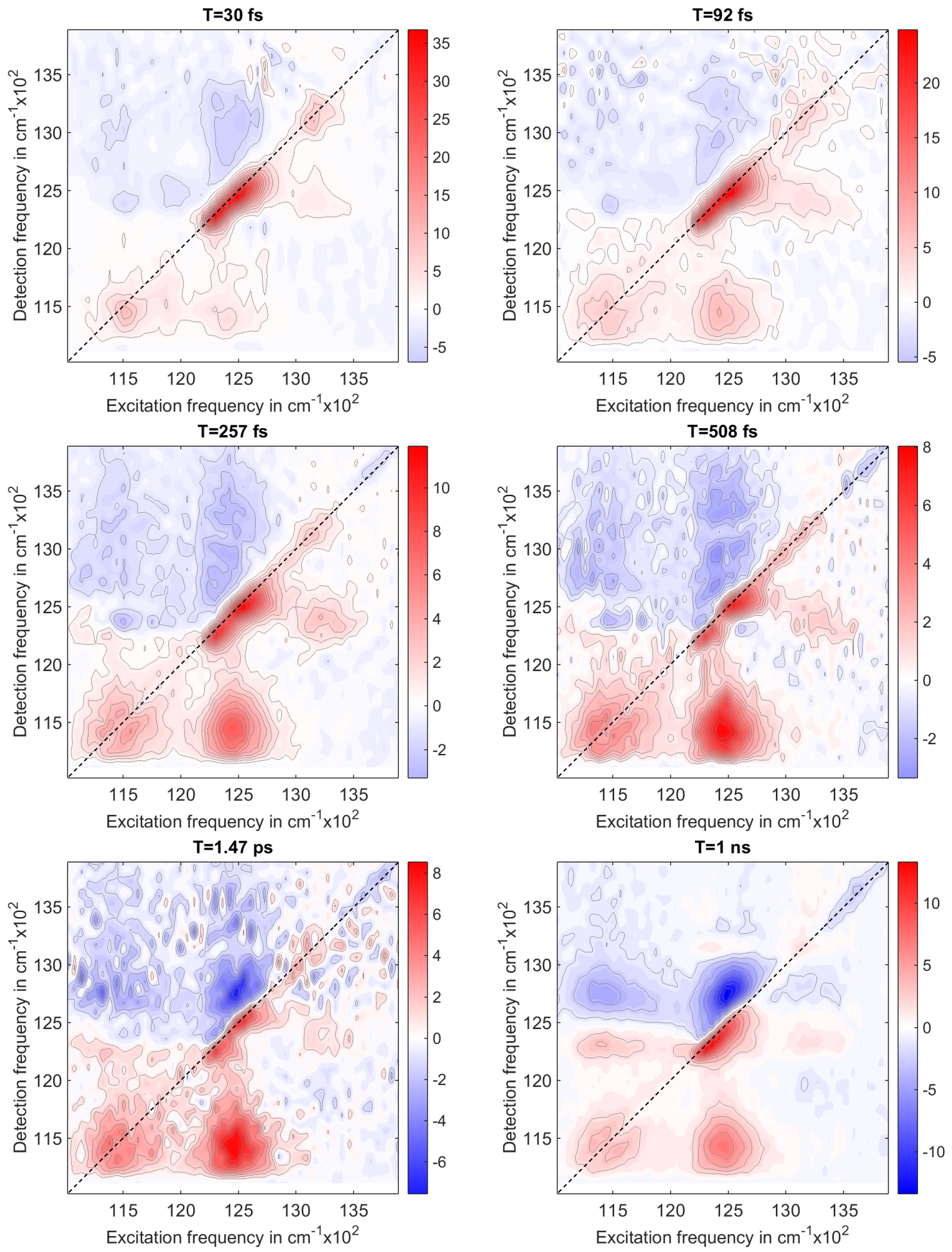

Figure 4.4: $S_{z}$ polarization associated spectra from $\mathrm{W}(\mathrm{M} 250) \mathrm{V}$ at $77 \mathrm{~K}$ 
uct state. Since $\mathrm{W}(\mathrm{M} 250) \mathrm{V}$ lacks $\mathrm{Q}_{A}$, we can assign this final state to $\mathrm{P}^{+} \mathrm{H}_{A}^{-}$, which decays on a $20 \mathrm{~ns}$ timescale [152] that is slower than we can accurately resolve in a 1 ns scan.

Figure 4.3 and Figure 4.4 show the $S_{y}$ and $S_{z}$ polarization associated 2D spectra described in Section 3.3. The $S_{y}$ component suppresses signals from probed transition dipoles which are parallel to the pumped transition dipole, reducing the amplitude of diagonal SE and GSB peaks from excitation and detection of the same state. At 30fs, the H-band diagonal peak is completely suppressed, while the $\mathrm{H} / \mathrm{B}_{A}$ cross-peak is visible due to the different dipole orientations between the two states. The most pronounced difference between $S_{y}$ and $S_{z}$ spectra is around the $\mathrm{B}$-band diagonal, with the $\mathrm{B}_{A} / \mathrm{B}_{B}$ cross-peaks appearing exclusively in the $S_{y}$ components.

\subsubsection{The DLL Mutant}

2D spectra from the DLL mutant with magic angle polarization are shown in Figure 4.5. Since DLL lacks the A-branch bacteriopheophytin, the charge-separated $\mathrm{P}^{+} \mathrm{H}_{A}^{-}$state never forms, so the final product state after energy transfer to $\mathrm{P}$ should be the excited $\mathrm{P}^{*}$ state, or possibly $\mathrm{P}^{+} \mathrm{B}_{A}^{-}$. Examining the P-excitation band in the first $100 \mathrm{fs}$, the cross-section along the detection axis changes very little over the course of the 1ns scan, indicating that this is in fact the $\mathrm{P}^{*}$ state. This assignment is consistent with the transient absorption $\mathrm{P}^{*}$ spectrum from Brederode et al. [35] and Zinth et al. [5], which both show a strong 880nm bleach, with a sharp ESA band at 806nm and a broad ESA feature extending beyond 750nm. Within a few hundred femtoseconds, the B-excitation spectrum resembles the same $\mathrm{P}^{*}$ state. Isolating the features of the $\mathrm{P}^{*}$ state proves to be helpful later on for kinetic fitting of the W(M250)V data, which we expect to have a similar $\mathrm{P}^{*}$ difference spectrum.

Excitation of the H-band produces a much weaker signal than in W(M250)V, which can be attributed entirely to $\mathrm{H}_{B}$ due to the lack of $\mathrm{H}_{A}$ in DLL. Unlike in W(M250)V, DLL has only one $\mathrm{H} / \mathrm{B}$ cross-peak from $\mathrm{H}_{B} \rightarrow \mathrm{B}_{B}$, which is further evidence that the two $\mathrm{H} / \mathrm{B}$ cross-peaks in $\mathrm{W}(\mathrm{M} 250) \mathrm{V}$ are from the two branches, and not $\mathrm{B}$ and $\mathrm{P}^{*}$. 

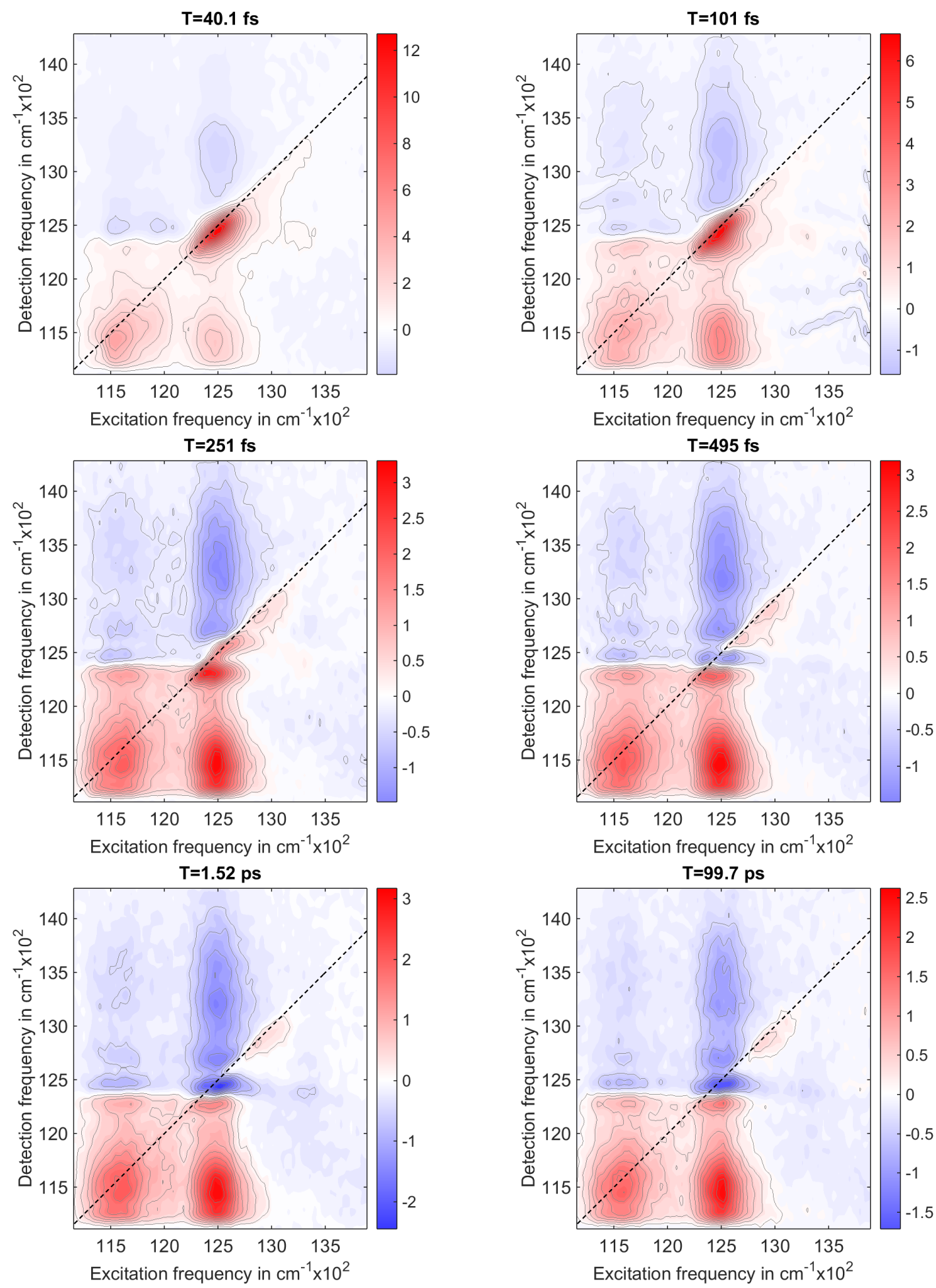

Figure 4.5: Real absorptive 77K 2DES spectra from DLL mutant BRCs with magic angle polarization 


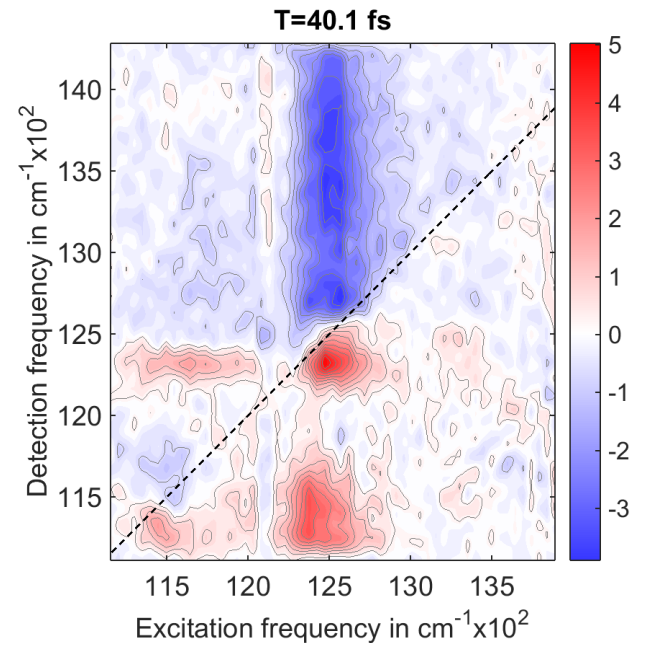

$\mathbf{T}=\mathbf{2 5 1}$ fs

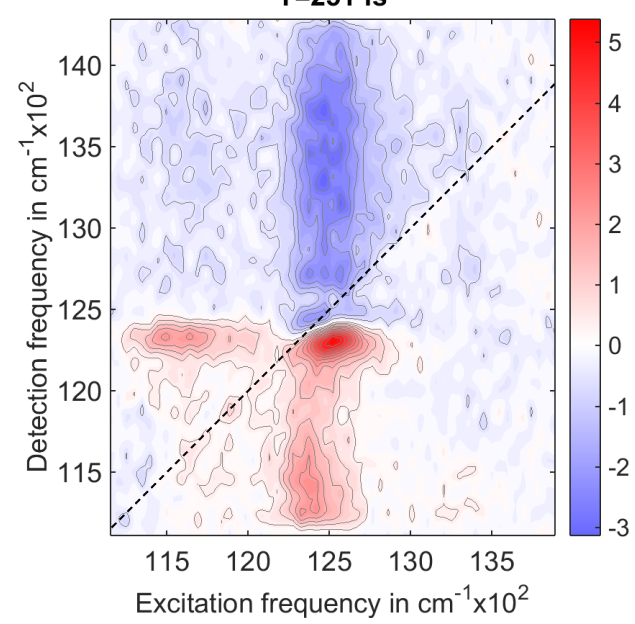

$\mathrm{T}=1.52 \mathrm{ps}$

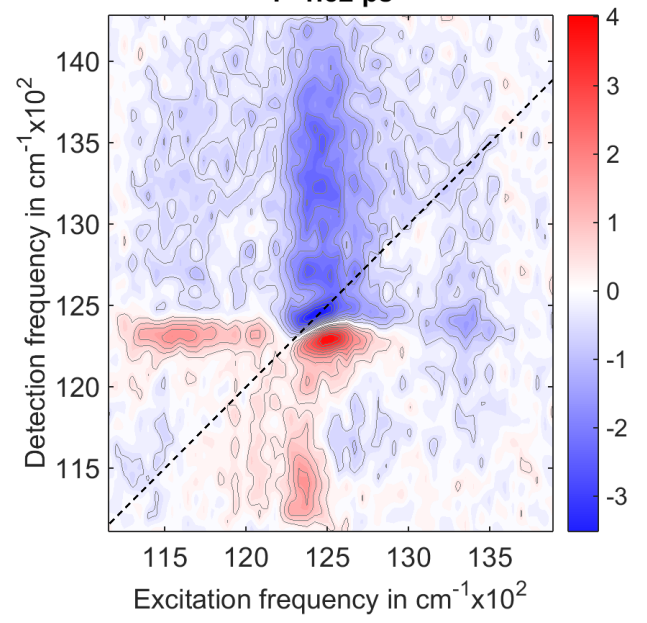

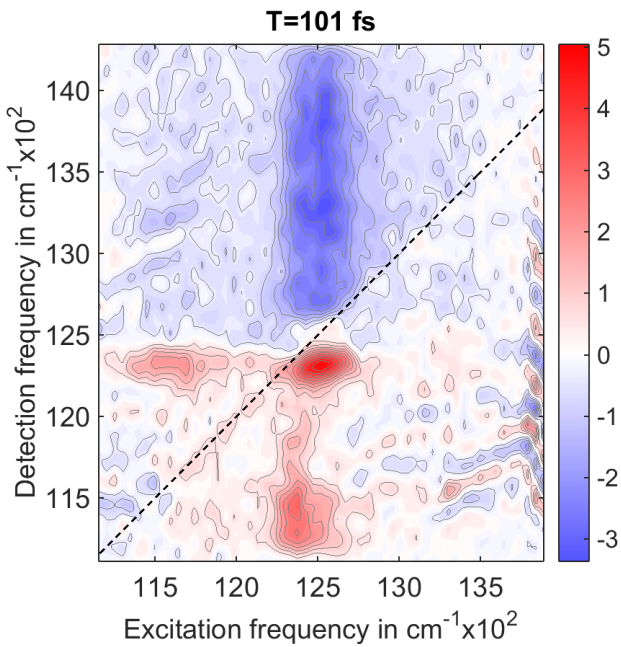

$\mathrm{T}=495 \mathrm{fs}$

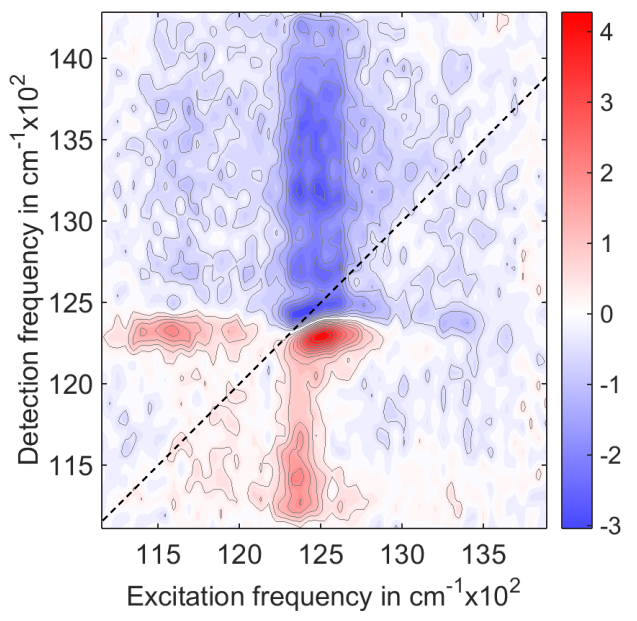

$\mathrm{T}=99.7 \mathrm{ps}$

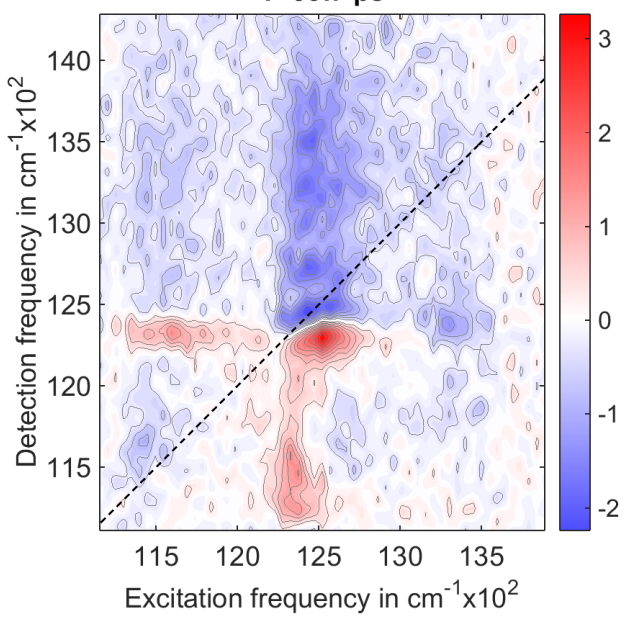

Figure 4.6: $S_{y}$ polarization associated spectra from DLL mutant at $77 \mathrm{~K}$ 


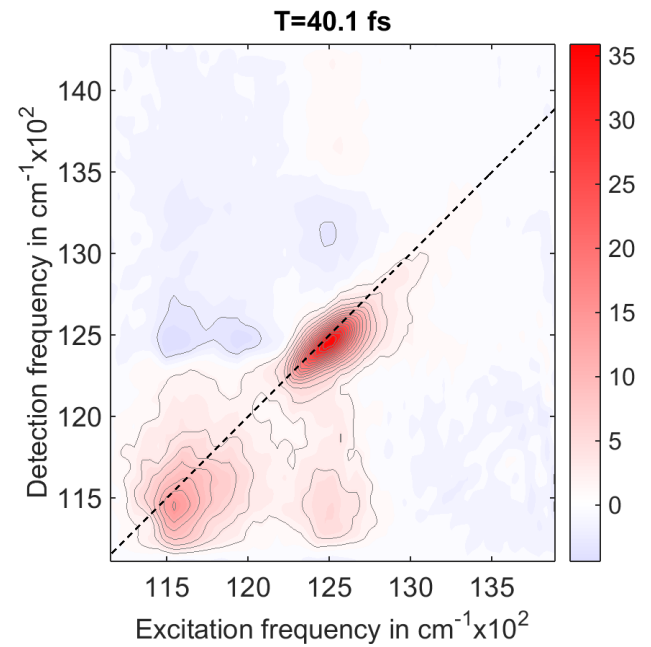

$\mathbf{T}=\mathbf{2 5 1} \mathrm{fs}$

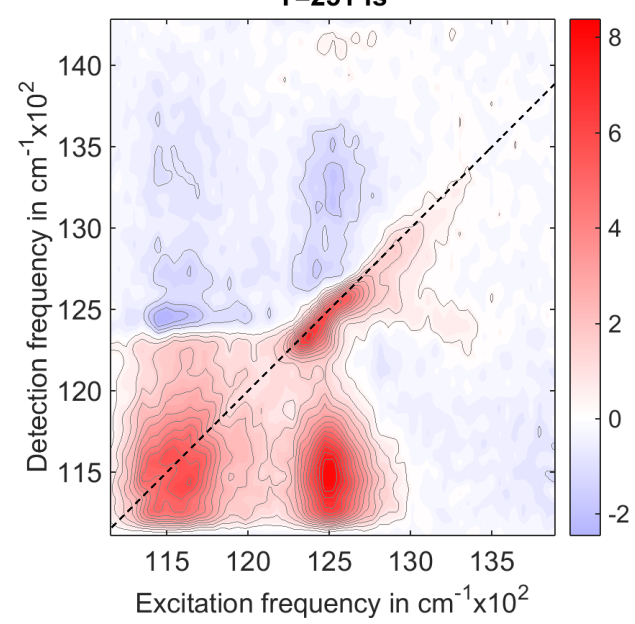

$\mathrm{T}=1.52 \mathrm{ps}$

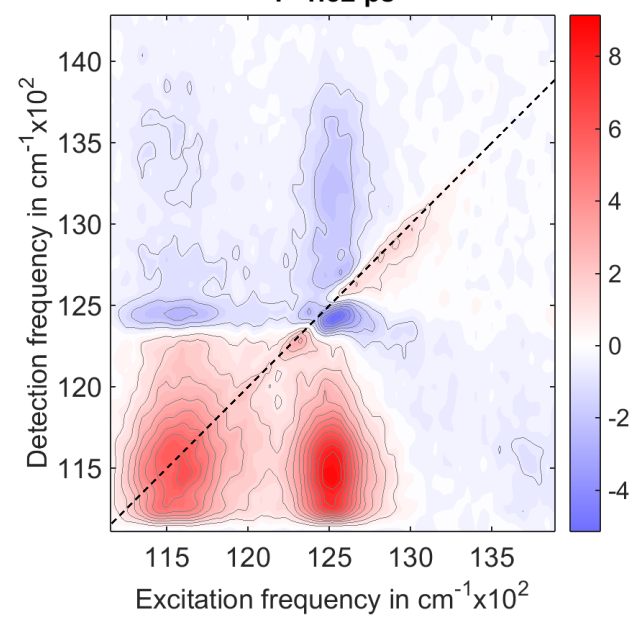

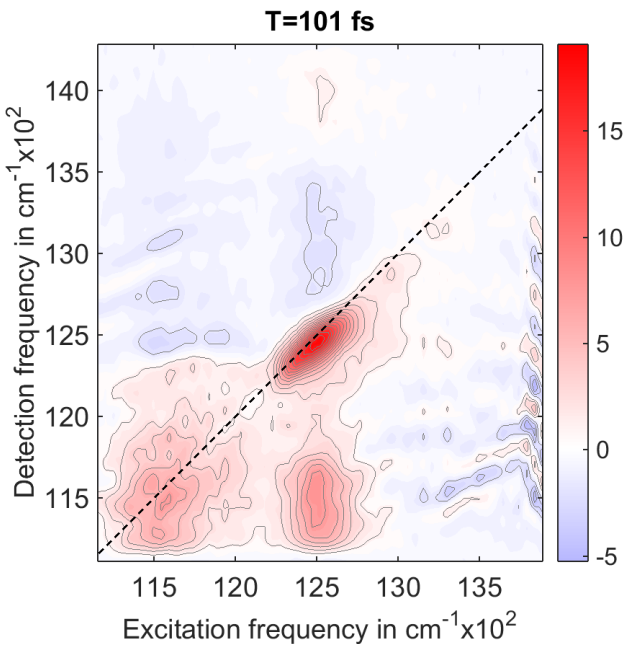

$\mathrm{T}=495 \mathrm{fs}$

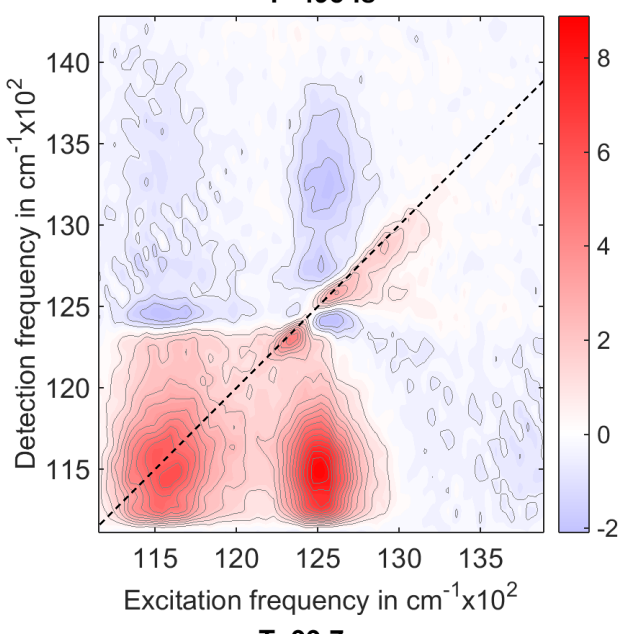

$\mathrm{T}=99.7 \mathrm{ps}$

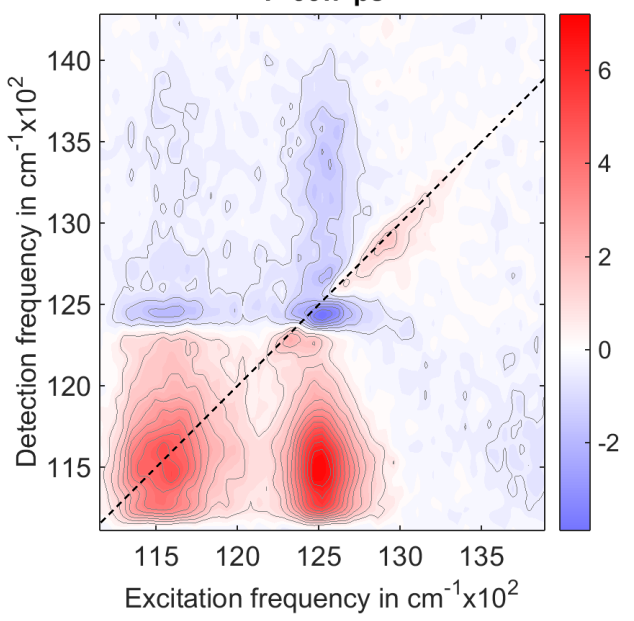

Figure 4.7: $S_{z}$ polarization associated spectra from DLL mutant at $77 \mathrm{~K}$ 
Figure 4.6 and Figure 4.7 show the 2D polarization associated spectra from DLL. The $S_{y}$ spectrum shows little change over the entire scan range. The B-excitation band of $S_{y}$ at $40 \mathrm{fs}$ has a spectrum resembling the final $P^{*}$ state, but missing the narrow negative band at $12500 \mathrm{~cm}^{-1}$. This band grows in within $250 \mathrm{fs}$, after which the spectrum remains unchanged. The timescales involved are consistent with $\mathrm{B} \rightarrow \mathrm{P}_{+} \rightarrow \mathrm{P}_{-}$energy transfer, which Jonas et al. found to occur with respective time constants of $80 \mathrm{fs}$ and 160fs [61]. The $S_{z}$ spectra have a strongly inhomogeneous B-band bleaching on the diagonal which decays within $500 \mathrm{fs}$ as the $\mathrm{P}^{*}$ state forms.

\subsection{DDAS and 2DEAS Fitting}

Two-dimensional Decay Associated Spectra and Species Associated Spectra were calculated using the procedure described in Section 3.2.1. In both cases, the only free parameters are the rate constants, as the initial conditions for the compartments are assumed implicitly for these models. This type of decomposition provides qualitative information about which parts of the 2D spectrum vary on different timescales.

The 2DDAS model has the fewest degrees of freedom, and converges to a unique solution for a given number of compartments. To determine how many states to use, several models were tested with different numbers of compartments, and the optimal rates for each model were found using a nonlinear least squares optimization algorithm. The initial guess for the $N$ rate constants was spaced logarithmically from $10 \mathrm{fs}$ to $1 \mathrm{~ns}$. The quality of the fit for each model was judged based on the total residual, the structure of the residual 2D spectra at representative population times from $<100 \mathrm{fs}$ to $1 \mathrm{~ns}$, and the amplitudes of the optimized basis spectra.

\subsubsection{W(M250)V 2DDAS Fitting}

Models with $N$ ranging from 2 to 7 states were tested, with the marginal improvement in residual becoming small after $N=4$. Figure 4.8(A) shows the computed basis spectra for $\mathrm{W}(\mathrm{M} 250) \mathrm{V}$ with the 4-compartment model, giving optimized decay rates of $81 \mathrm{fs}$, 171fs, 

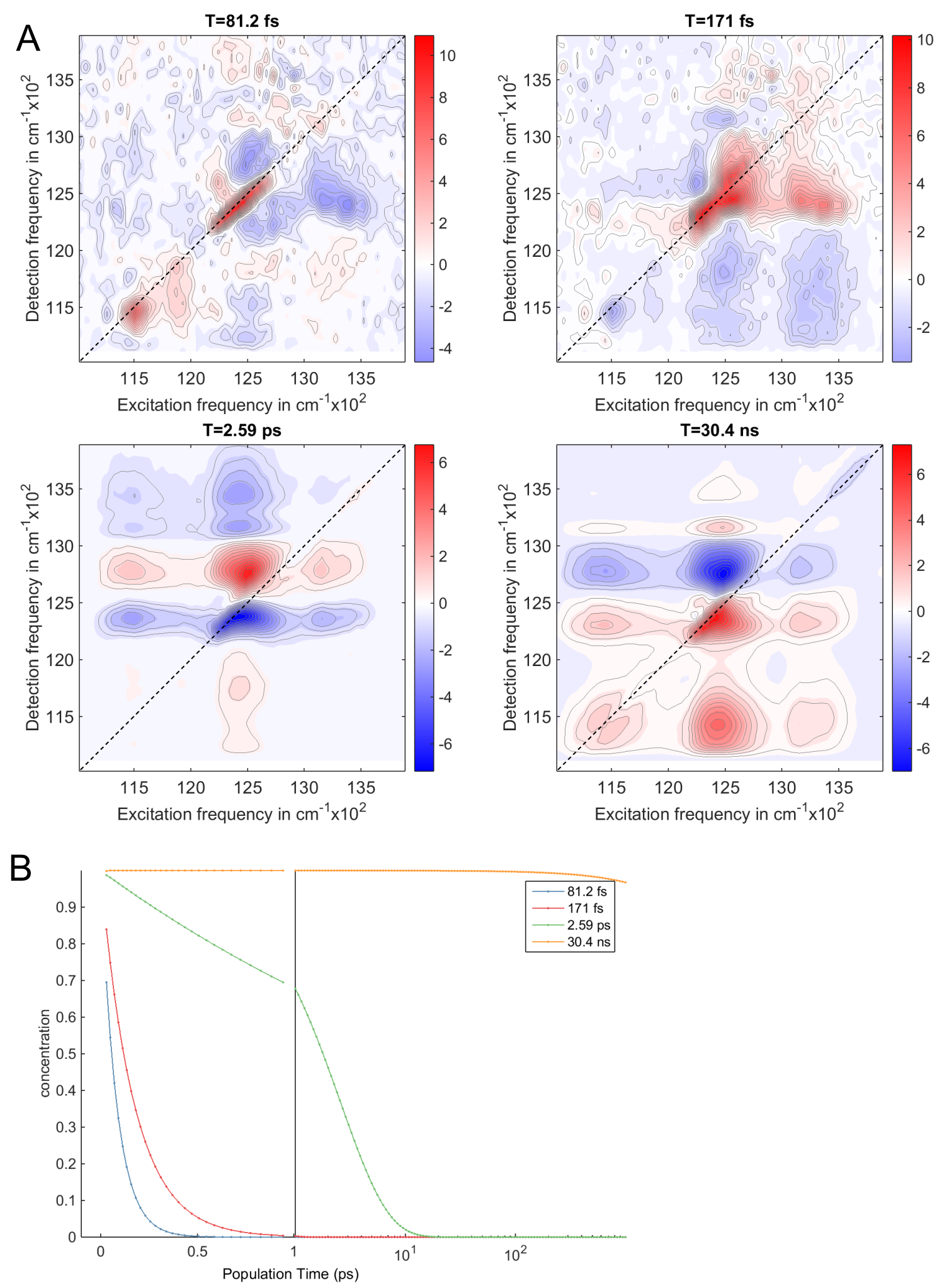

Figure 4.8: (A) 4-compartment 2DDAS basis spectra from W(M250)V magic angle data at 77K. (B) Time-dependent concentrations of the basis spectra, plotted on a split linear $/ \log$ arithmic scale. 
2.6ps, and 30ns, and Figure 4.8(B) shows the associated time-dependent concentrations of each compartment. The $81 \mathrm{fs}$ component reveals several features that were not easily visible in the absorptive 2D spectra shown in Figure 4.2. A weak positive diagonal peak appears at $11800 \mathrm{~cm}^{-1}$ which matches the position of the upper excitonic level $\mathrm{P}_{+}$based on fitting of the $77 \mathrm{~K}$ linear absorption spectrum (see Figure 4.19). This position also agrees reasonably well with the $11970 \mathrm{~cm}^{-1}$ position of $\mathrm{P}_{+}$calculated from the crystal structure of $R$. viridis [16].

Note that many of the features appearing in each 2DDAS spectrum have complimentary features of inverse sign appearing in an adjacent DAS spectrum, so that the sum of the two cancel when the concentrations of each are comparable. This effect typically appears in DAS fitting sequential reactions. The DAS basis spectra only approximate the true difference spectra when the species are uncoupled, making this approach unsatisfactory for representing energy and charge transfer processes, even though the reconstructed 2D spectra fit the measured data well with only a few components. 2DDAS fitting of the DLL data (not shown here) exhibits similar mutually canceling linearly dependent basis spectra.

\subsubsection{W(M250)V 2DEAS Fitting}

2DEAS fitting with a sequential kinetic model can produce basis spectra which are more reasonable approximations of difference spectra from actual states of the system. For a given number of compartments, both 2DDAS and 2DEAS have the same number of free rate parameters (assuming no branching), but even with only a few parameters, 2DEAS optimization runs into uniqueness problems. With 2DDAS, the order of the $N$ decay rates is arbitrary, since the initial conditions of each compartment are the same. For 2DEAS, however, the order of the rates $\left\{k_{12}, k_{23} \ldots k_{\mathrm{NN}}\right\}$ matters, and as discussed in Section 3.1.2, permutations of the rates $\left\{k_{i}\right\}$ will result in identical residuals but drastically different SADS. For the BRC data, a reasonable first guess for an unbranched sequential model might be to order the rates as $k_{1}>k_{2}>\ldots>k_{N}$ (or $\tau_{12}<\tau_{23}<\ldots<\tau_{\mathrm{N}-1, \mathrm{~N}}<\tau_{\mathrm{NN}}$ ) to allow for fast energy transfer dynamics followed by picosecond charge separation. After finding the 

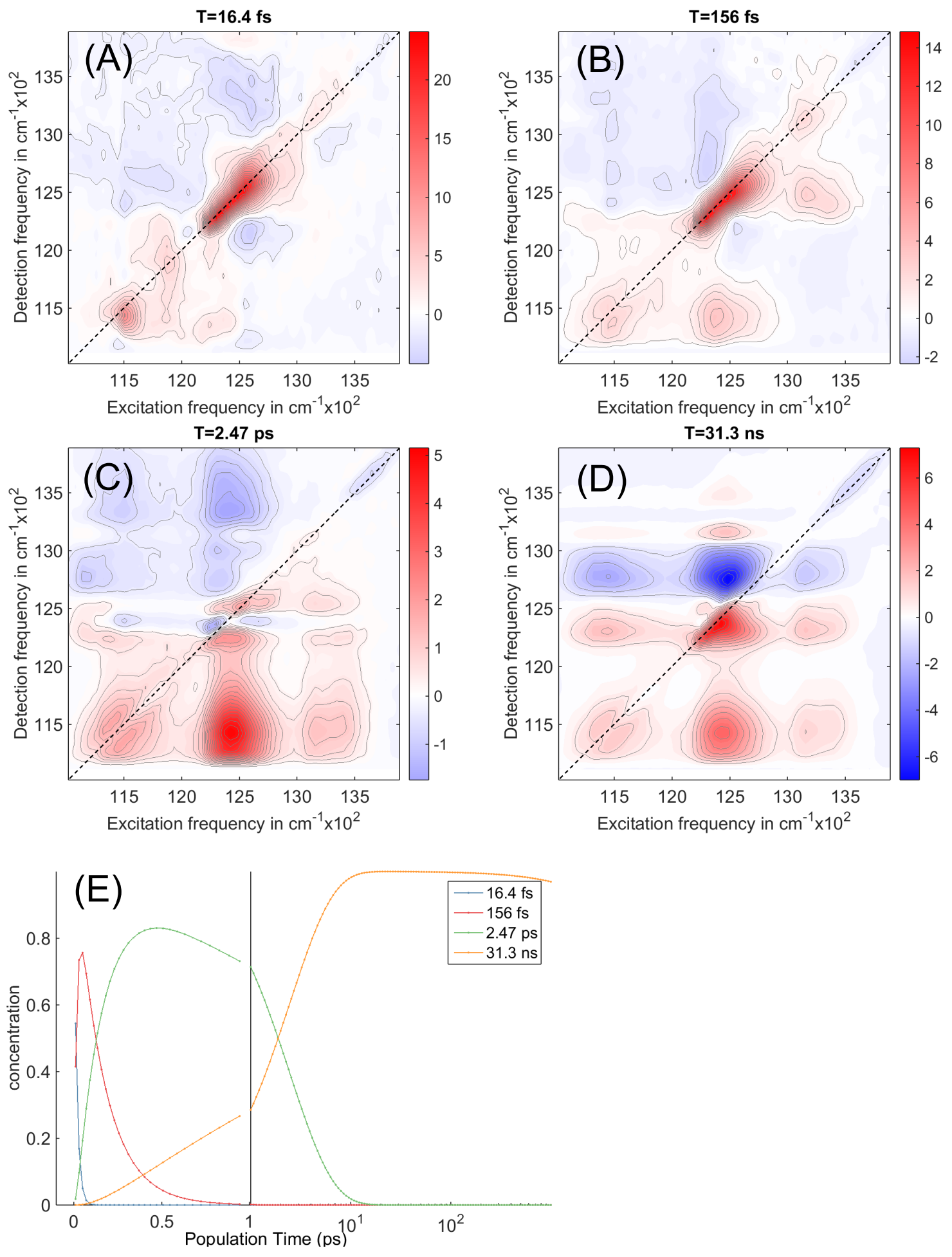

Figure 4.9: (A-D) 2DEAS spectra for $\mathrm{W}(\mathrm{M} 250) \mathrm{V}$ with magic angle polarization using a 4-compartment unbranched sequential model. (E) Time-dependent concentrations. 
optimal rates for an $N$-compartment model, one must then try different permutations of those rates and examine the features of the resulting basis spectra to choose the most reasonable model. This ambiguity inherent in sequential models has led to inconsistent conclusions in the literature regarding the rates involved in the $\mathrm{P}^{*} \rightarrow \mathrm{P}^{+} \mathrm{H}_{A}^{-} \rightarrow \mathrm{P}^{+} \mathrm{B}_{A}^{-}$sequence, and will be discussed in more detail in Section 4.3.

Figure 4.9 shows the basis spectra for a 4-compartment sequential model from the W(M250)V data. The optimized timescales are similar to those found in the 2DDAS case, and the spectrum of the first EAS compartment shares similar features to the first DAS compartment, since the concentration of the first compartment decays as $1 / \tau_{1}$ in both models. The 16.4fs EAS compartment (Figure 4.9A) has the same diagonal peak at $11900 \mathrm{~cm}^{-1}$ with a cross-peak at the $\mathrm{P}_{-}$detection frequency $11500 \mathrm{~cm}^{-1}$. The $156 \mathrm{fs}$ component (Figure 4.9B) shows growth of the $\mathrm{H} / \mathrm{B}$ and $\mathrm{B} / \mathrm{P}$ cross-peaks, indicating that energy transfer has begun within 10's of femtoseconds. Comparing the final amplitudes of these cross-peaks after several hundred femtoseconds in Figure 4.2, the B/P cross peak has much higher amplitude than $\mathrm{H} / \mathrm{B}$, while they are comparable in the 156fs compartment. $\mathrm{H} \rightarrow \mathrm{B}$ grows in faster than $\mathrm{B} \rightarrow \mathrm{P}$, but the 2DEAS decomposition does a poor job of distinguishing these rates. All we can conclude from (Figure 4.9B) is that some energy transfer has occurred on $<100 \mathrm{fs}$ timescale, and we must fit the kinetics of the individual excitation bands to extract the individual rates.

Figure $4.9 \mathrm{C}$ shows an intermediate state with a $2.5 \mathrm{ps}$ lifetime, and cross-sections of the detected spectrum are mostly independent of the excitation frequency. On this timescale, the excitation-dependent energy transfer processes have mostly finished, and a common intermediate state has formed. Likewise, Figure 4.9D looks like the final $\mathrm{P}^{+} \mathrm{H}_{A}^{-}$state independent of excitation. Due to the lack of excitation dependence, spectra (C) and (D) resemble real basis spectra for the system, while (A) and (B) only qualitatively represent the energy transfer dynamics. 


\subsubsection{DLL 2DEAS Fitting}

For the DLL data, a 3-state sequential model reproduces the data reasonably well. Adding additional compartments does improve the residual, but the added basis spectra are low-amplitude and nearly linearly dependent relative to the 3-state spectra. The resulting 2DEAS and their associated concentrations are shown in Figure 4.10. The first two compartments show similar information as Figure 4.9A-B from W(M250)V. (A) shows the lineshapes associated with initial excitation of $\mathrm{B}$ and $\mathrm{P}$, and an early $\mathrm{B} / \mathrm{P}$ cross-peak, and (B) indicates $\mathrm{H}_{B} \rightarrow \mathrm{B}_{B}$ and $\mathrm{B} \rightarrow \mathrm{P}$ energy transfer on timescales on the order of 100fs, but the individual rates cannot be extracted solely with the 2DEAS decomposition. (C) shows the spectrum of the same $\mathrm{P}^{*}$ state discussed from Figure 4.5.

Figure 4.11 demonstrates the effect of choosing the wrong order for the rate constants in a sequential model. The DLL fitting presents an example with very few free parameters in which one of the six possible permutations of rate constants is clearly the best model. The basis spectra and concentrations shown in Figure 4.11 give an identical residual to the model in Figure 4.10, despite the order of the rate constants being reversed. Note that the concentrations of the second and third compartments are negligibly small, but the basis spectra are orders of magnitude larger, so that the product of the two has a noticeable effect

on the fit. The three basis spectra are very close to being linearly dependent, and the finer features in the data are reproduced by subtle differences in the basis spectra, multiplied by negligibly small scaling factors. This is an extreme example of the types of effects that can appear in a poorly constrained global fit, and what we seek to avoid in the multi-excitation fitting described in Section 4.4.

In summary, the 2DDAS and 2DEAS fitting has shown us several things: (1) The improvement to the quality of fit becomes small after 4 compartments for the $\mathrm{W}(\mathrm{M} 250) \mathrm{V}$ data, and 3 compartments for the DLL. Resolving more time constants that this requires additional information, which the multi-excitation fitting provides by imposing model-specific co-dependences between the columns of the 2D spectra. (2) The picosecond and nanosecond 

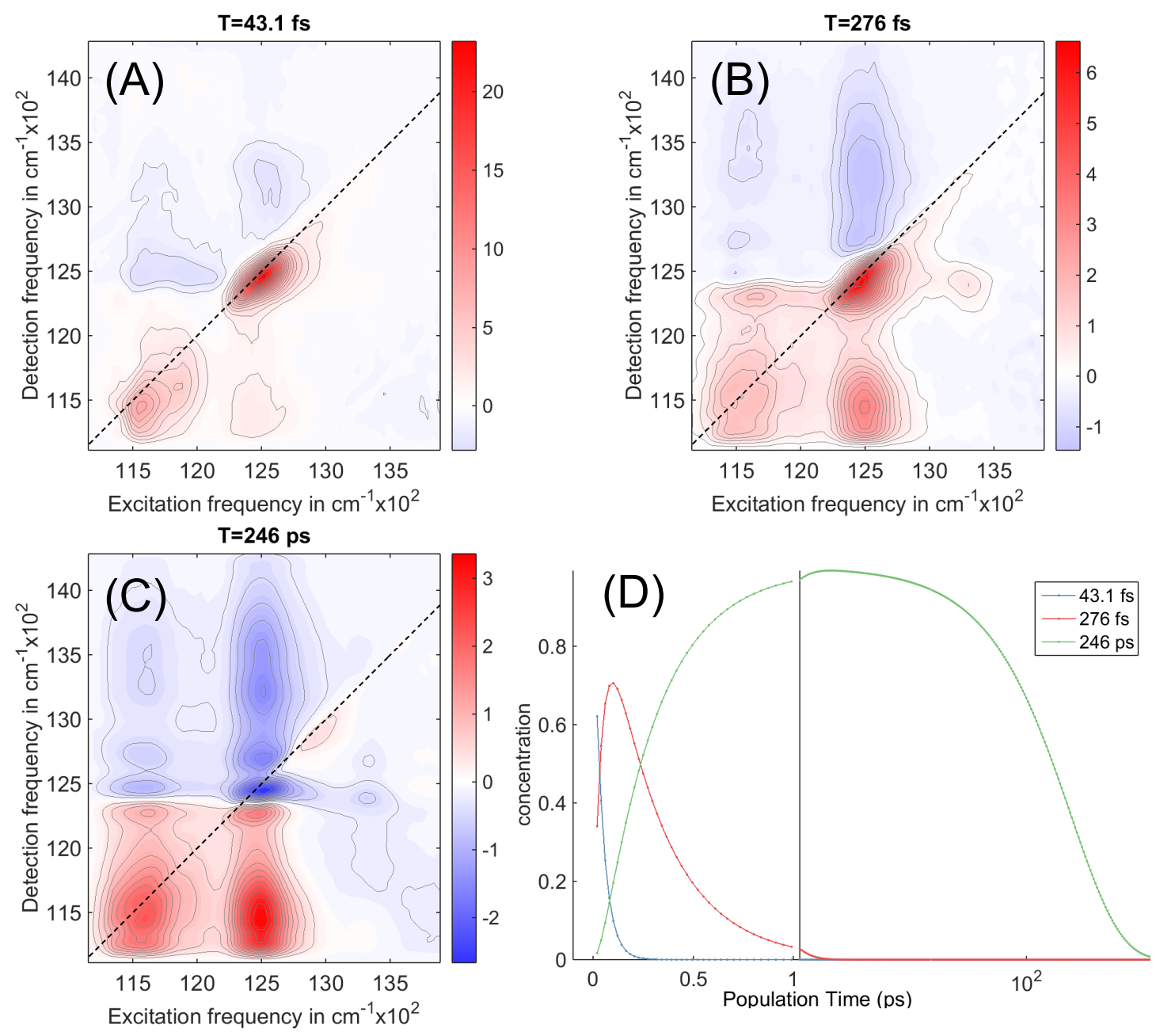

Figure 4.10: (A-C) 2DEAS spectra for DLL using a 3-compartment unbranched sequential model. (B) Time-dependent concentrations. 

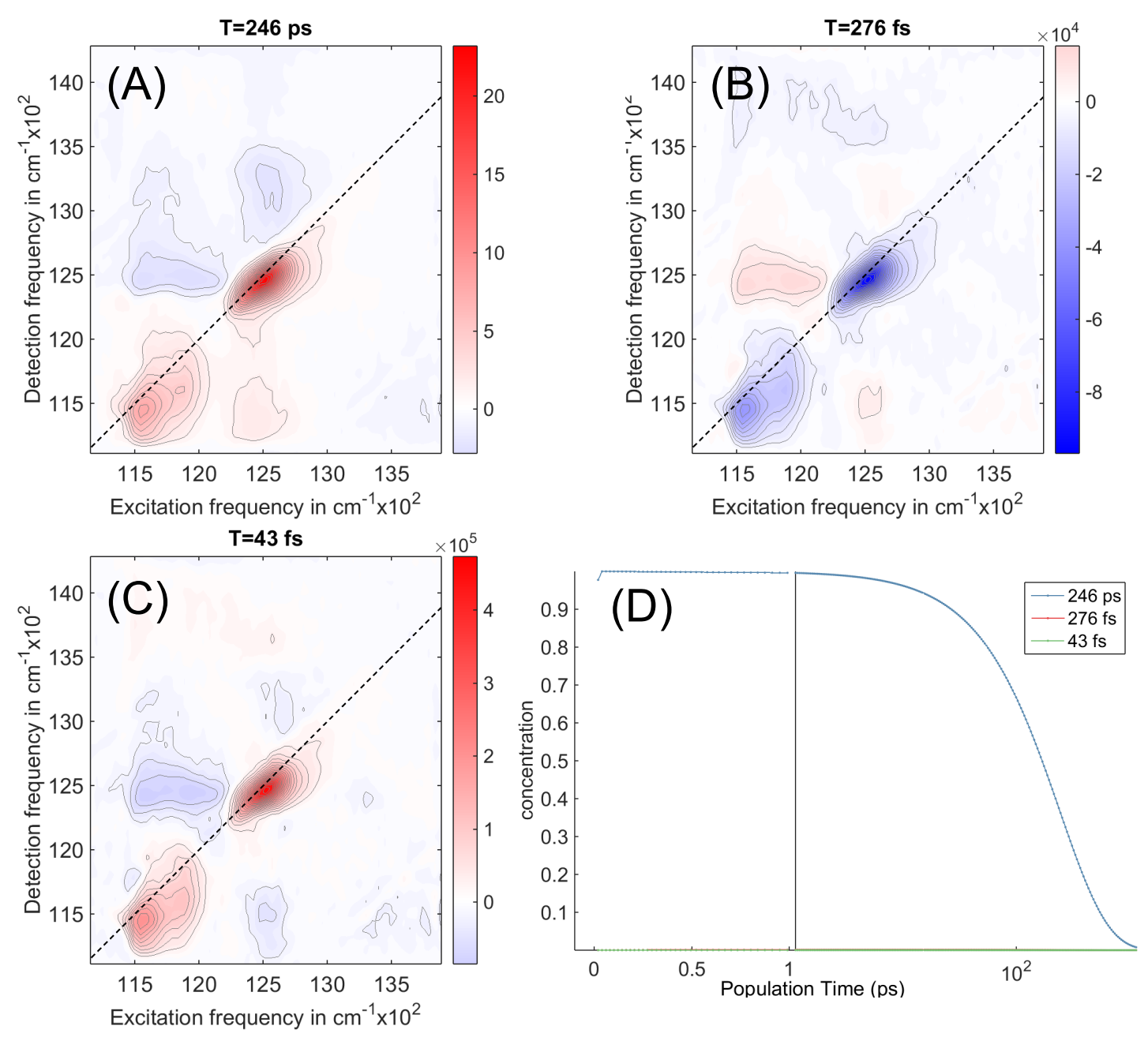

Figure 4.11: (A-C) 2DEAS spectra for DLL using a 3-compartment model with the same rates as Figure 4.10 in reverse order. (B) Time-dependent concentrations. 
timescale compartments show 2D spectra which are mostly excitation-independent, with the exception of the inhomogeneous broadening on the B-band diagonal in W(M250)V. This B-band inhomogeneity presents an additional challenge in the multi-excitation fitting. (3) Best-fit timescales for the overall evolution of the $2 \mathrm{D}$ spectrum. These values provide a reasonable initial guess for the gradient-descent optimization in Section 4.4. (4) We demonstrated how the 2DEAS fit suffers from the same uniqueness problem as SADS fitting of TA spectra (Figure 4.11). Even with the more physically reasonable model in Figure 4.10, the $2 \mathrm{D}$ basis spectra are not even approximately equal to $2 \mathrm{D}$ spectra from individual species.

\subsection{Fitting of Individual Excitation Bands}

Cross-sections of the absorptive 2DES spectrum at specific excitation frequencies are directly comparable to TA experiments with a corresponding narrow-bandwidth pump. Therefore, examining the kinetic fitting results from the separate excitation bands is useful for checking the consistency of our results with previous studies, to reproduce and test the uniqueness of specific proposed kinetic models using a comparable data set, and as a control with which to compare the results of the multi-excitation 2DES fitting presented in Section 4.4. Fitting each excitation frequency separately, with independent time constants, is less constrained than the 2DEAS fit, which required all excitation frequencies to share one set of rates. However, given that each excitation frequency excites different populations which proceed with different sequential reactions, it is not necessarily reasonable to assume the same number of compartments and common rates at all excitation frequencies, as 2DEAS fitting does. For example, an 880nm excitation may initiate the sequence $\mathrm{P}^{*} \rightarrow \mathrm{P}^{+} \mathrm{B}_{A}^{-} \rightarrow \mathrm{P}^{+} \mathrm{H}_{A}^{-}$, while 760nm excitation initiates $\mathrm{H} \rightarrow \mathrm{B} \rightarrow \mathrm{P}^{*} \rightarrow \mathrm{P}^{+} \mathrm{B}_{A}^{-} \rightarrow \mathrm{P}^{+} \mathrm{H}_{A}^{-}$. Representing the former sequence with the same 5-compartment scheme as the latter would not yield physically meaningful results.

Brederode et al. [35] performed excitation wavelength-dependent TA measurements on wild-type Rhodobacter sphaeroides reaction centers at $77 \mathrm{~K}$. The reported instrument response function for these experiments was $350 \mathrm{fs}$, which is an order of magnitude longer than 
in our 2D measurements. Despite this difference, the picosecond kinetics and product state difference spectra should be consistent between the pump-probe and 2D measurements. Below, we show "transient absorption" spectra extracted from the 2DES data at the specific excitation frequencies shown in Figure 4.12, and compare global fits of each excitation band to the results from Brederode et al.
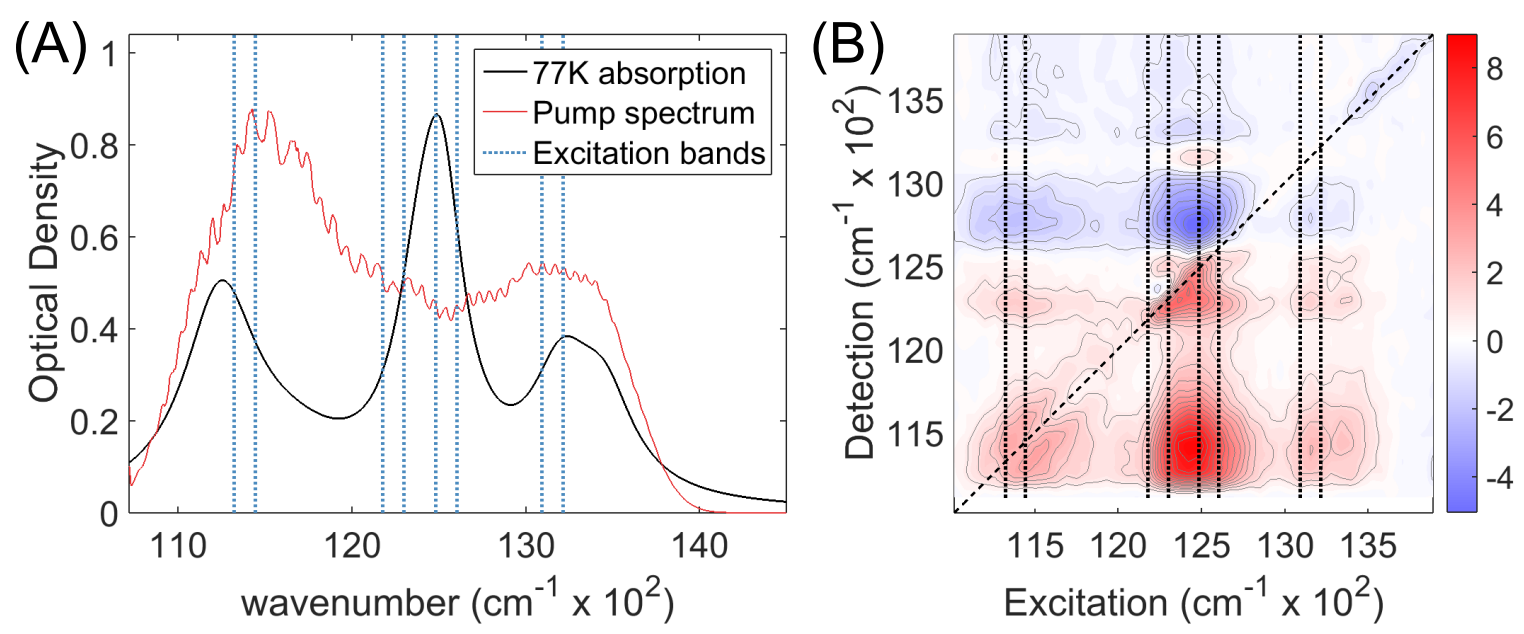

Figure 4.12: (A) 77K linear absorption spectrum from $\mathrm{W}(\mathrm{M} 250) \mathrm{V}$, the pump spectrum used for the 2DES experiments, and the four excitation bands selected for kinetic fitting, corresponding to $865 \mathrm{~nm}, 818 \mathrm{~nm}, 796 \mathrm{~nm}$, and $760 \mathrm{~nm}$. (B) Excitation bands projected onto a representative $2 \mathrm{D}$ spectrum at $\mathrm{T}=2 \mathrm{ps}$

Plots (A-C) of Figure 4.13 show the SADS derived from the 2DES data at the listed excitation wavelengths, using the rate constants reported by Brederode et al. Plots (DF) show the published SADS from [35] with the same excitation wavelengths, except that we selected $865 \mathrm{~nm}$ rather than $880 \mathrm{~nm}$ to stay within the spectrally flat part of our pump bandwidth. For all three excitation wavelengths, there is little difference between the last two components (assigned as $\mathrm{P}^{+} \mathrm{H}_{A}^{-}$and $\mathrm{P}^{+} \mathrm{Q}_{A}^{-}$), which is expected since the $\approx 80 \mathrm{ps}^{+} \mathrm{H}_{A}^{-} \rightarrow$ $\mathrm{P}^{+} \mathrm{Q}_{A}^{-}$is absent in $\mathrm{W}(\mathrm{M} 250) \mathrm{V}$. The first three SADS of (A-C) and the $\mathrm{P}^{+} \mathrm{H}_{A}^{-}$components in (A-B) match the corresponding spectra in (D-F). The 1.7ps and $2 \mathrm{ps}$ components in (B) and $(\mathrm{C})$, respectively, show the same P-band bleaching and B-band derivative line shapes as in [35], but the relative amplitudes of the peaks in the B-band are not consistent. These SADS were assigned as mixtures of $\mathrm{P}^{*}$ and $\mathrm{P}^{+} \mathrm{B}_{A}^{-}$in [35]. The inconsistency could be due 

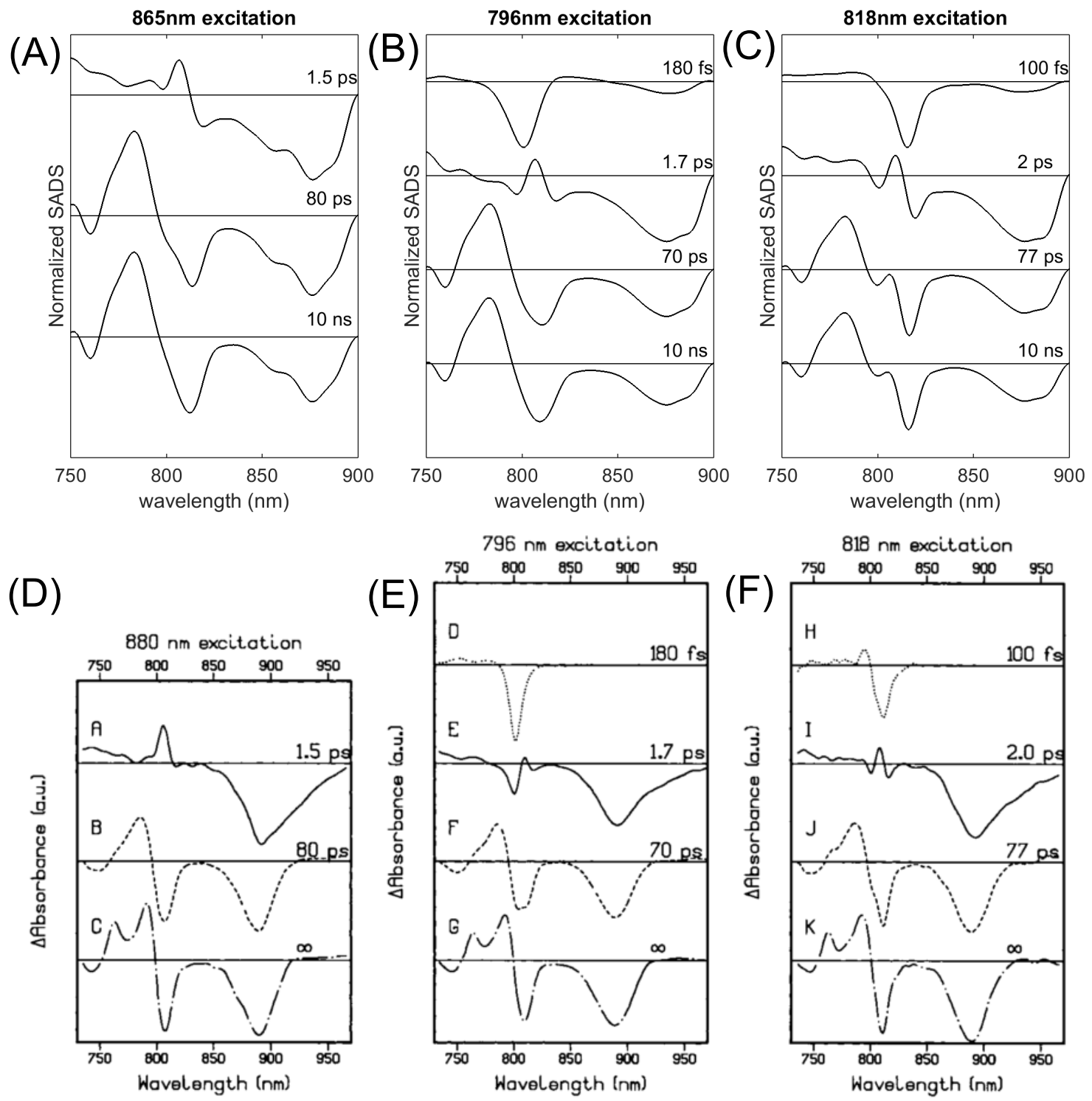

Figure 4.13: SADS derived from cross-sections of W(M250)V 2DES spectra at specific excitation wavelengths. (A-C) The rate constants were fixed at the values reported by Brederode et al.[35]. (D-F) SADS published by Brederode et al.[35] corresponding to similar excitation wavelengths. 
to different ratios of $\mathrm{P}_{+}$and $\mathrm{B}$ being excited at these specific wavelengths in wild-type $R$. sphaeroides vs $\mathrm{W}(\mathrm{M} 250) \mathrm{V}$, or from solvent-dependent shifts in the peak positions.

Another notable difference is in the $\mathrm{P}^{+} \mathrm{H}_{A}^{-}$SADS with $818 \mathrm{~nm}$ excitation (77ps and 10ns components), which have distinctly split peaks at $800 \mathrm{~nm}$ and $815 \mathrm{~nm}$ not present in the $865 \mathrm{~nm}$ and $796 \mathrm{~nm}$ spectra. This feature is apparent in the $\mathrm{T}=1 \mathrm{~ns} 2 \mathrm{D}$ spectrum in Figure 4.2 as the diagonal peak and above-diagonal cross-peak in the B-band. A similar splitting of the B-band 77K difference spectra was observed in R. sphaeroides by Kirmaier et al. [103], which showed a strong dependence on the glycerol content of the sample. The $\mathrm{P}^{+} \mathrm{H}_{A}^{-}$difference spectrum with $56 \%$ glycerol showed distinct splitting of the B band similar to the $818 \mathrm{~nm}$ excitation in Figure 4.13 , while the $65 \%$ glycerol sample resembled the smoother $\mathrm{P}^{+} \mathrm{H}_{A}^{-}$spectrum shown in [35]. Our samples were in a 50/50 buffer/glycerol mixture, while Brederode et al. used $60 \%$ glycerol, which is consistent with the observations in [103]. With the exception of the B-band splitting, the SADS of the two final states in Figure 4.13(B-C) look very similar to each other and that of (A). Upon closer examination, the difference between these two SADS, shown in Figure 4.14, resembles a slightly asymmetric second-derivative line shape centered at $806 \mathrm{~nm}$. The $\mathrm{P}^{+} \mathrm{H}_{A}^{-}$spectrum is also similar at all other excitation wavelengths, including H-band excitation in Figure 4.16, and this feature persists from 2ps to the full 1ns scan range.

Figure 4.15 shows the least-squares optimized rates and SADS, using the rates in Figure 4.13A-C as initial conditions for the optimization. Since our data do not contain the $80 \mathrm{ps}^{+} \mathrm{H}_{A}^{-} \rightarrow \mathrm{P}^{+} \mathrm{Q}_{A}^{-}$component, the optimizer re-allocates those compartments to fit the faster energy transfer dynamics. The $36 \mathrm{fs}$ component in (A) might correspond to internal conversion between the special pair excitonic levels $\mathrm{P}_{+} \rightarrow \mathrm{P}_{-}$, estimated by Vos et al. to have a 50-100fs time constant [54]. The 865nm pump excites a mixture of $\mathrm{P}_{+}$and $\mathrm{P}_{-}$, and does not have significant overlap with B or H. The SADS in (B-C) correspond to excitation of different ratios of $\mathrm{B}_{A}$ and $\mathrm{B}_{B}$, with a small overlap with $\mathrm{P}_{-}$, so the rates associated with each compartment are effective rates from energy transfer from those different mixtures. 


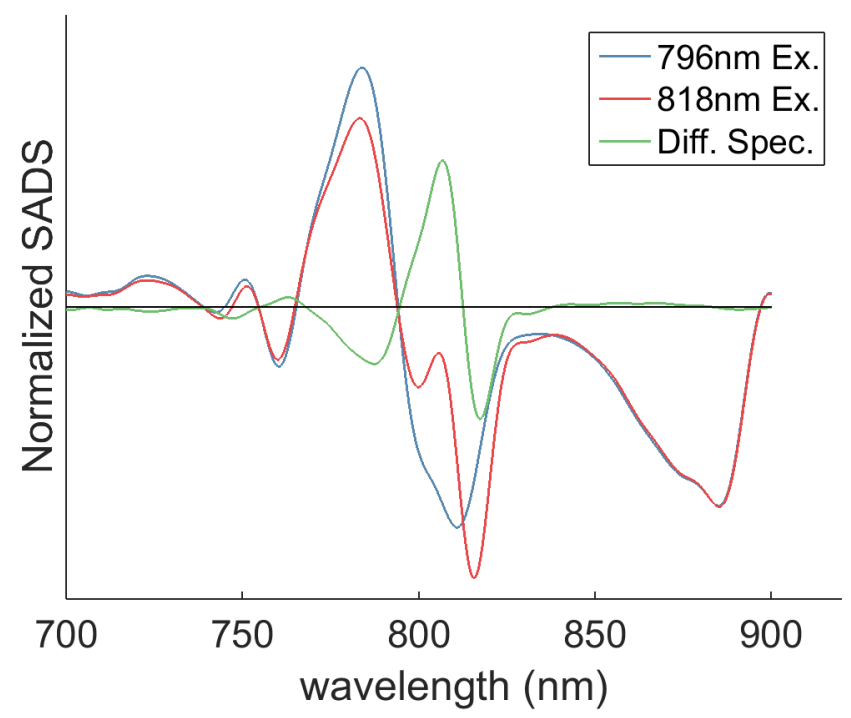

Figure 4.14: $\mathrm{P}^{+} \mathrm{H}_{A}^{-}$SADS from 796nm and 818nm excitation (blue and red respectively), normalized to the P-band bleaching. Plotted in green is the difference between the two normalized spectra.

\section{Optimized SADS}
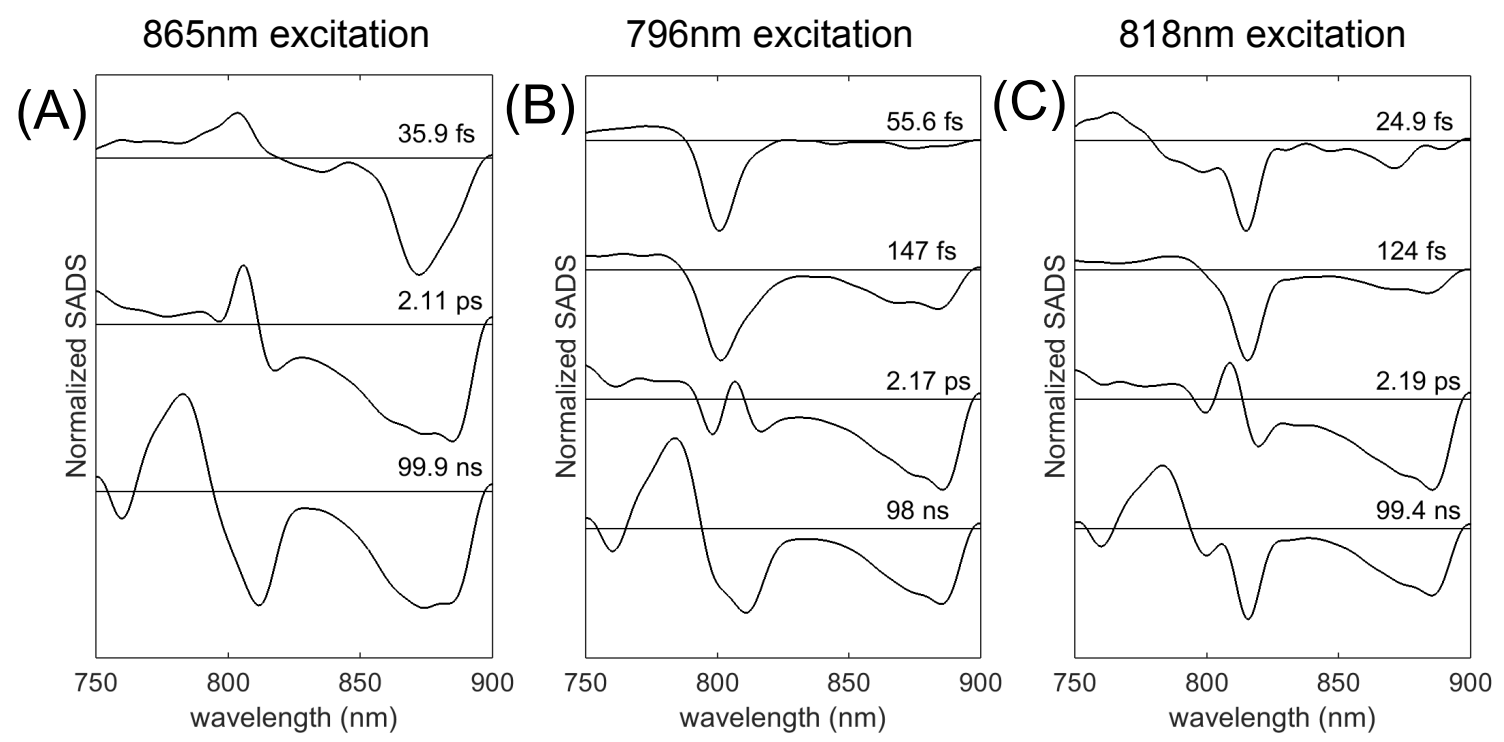

Figure 4.15: Optimized SADS and time constants corresponding to (A-C) of Figure 4.13 
The first two compartments of each have timescales consistent with $\mathrm{B} \rightarrow \mathrm{P}$ energy transfer between 150 and 200fs $[16,32,33,54]$, and the 2 ps compartments resemble mixtures of $\mathrm{P}^{*}$ and $\mathrm{P}^{+} \mathrm{B}_{A}^{-}[35]$. The sub-picosecond B-band kinetics are particularly difficult to disentangle for this reason.

760nm excitation
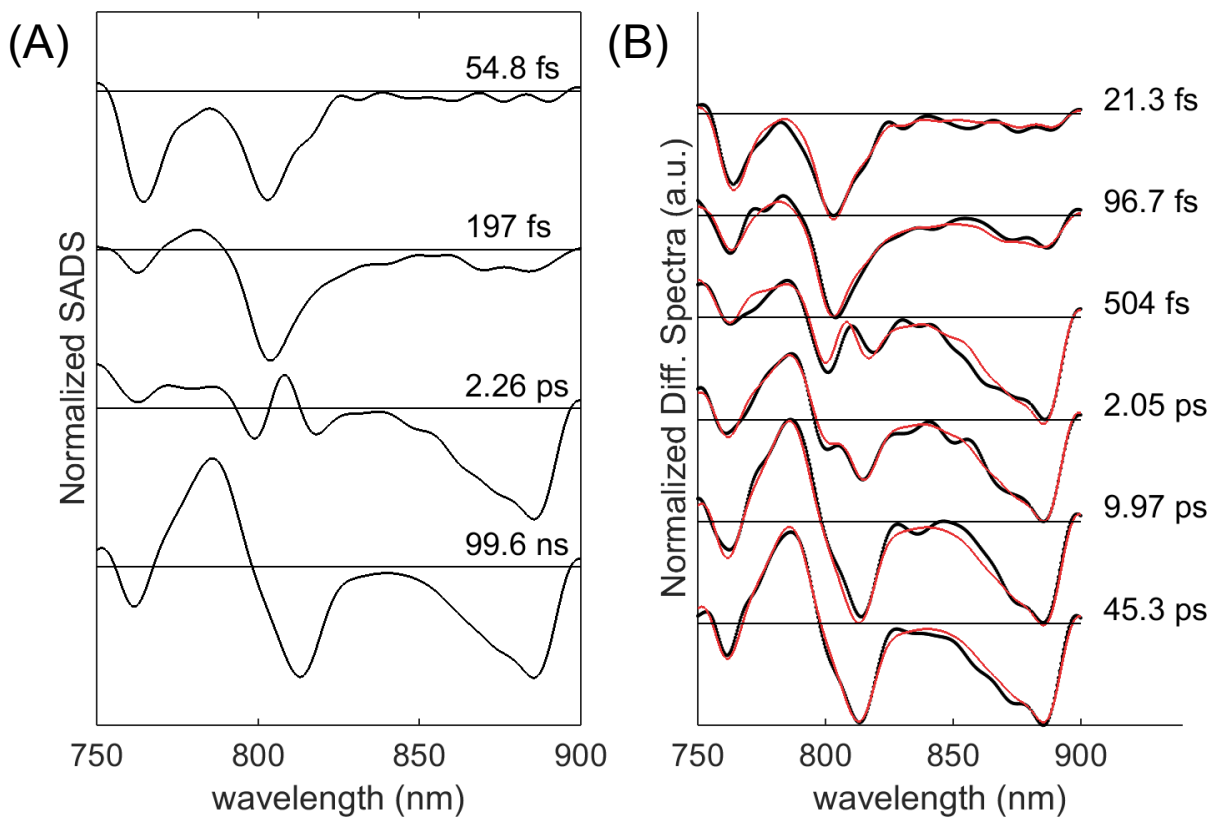

Figure 4.16: (A) 4-compartment SADS calculated from 760nm excitation of W(M250)V. (B) Measured spectra (black) and SADS fit (red) from slices of the 2DES spectrum at 760nm excitation and several population times.

Figure 4.16(A) shows the SADS fit from excitation of the H-band, and (B) shows traces of the H-band spectrum at several population times. Examining the early time traces reveals that the H/B cross-peak appears almost instantly after excitation. This is consistent with measurements by Vos et al. [54] and Lin et al. [153], who observed $\mathrm{H} \rightarrow \mathrm{B}$ energy transfer in $<100 \mathrm{fs}$. Photochemical hole-burning measurements on $R$. sphaeroides estimated $30 \mathrm{fs}$ lifetimes for both $\mathrm{H}_{A}$ and $\mathrm{H}_{B}$ [102]. The subsequent SADS are nearly identical to those of Figure 4.13E, corresponding to $\mathrm{B} \rightarrow \mathrm{P} \rightarrow \mathrm{P}^{+} \mathrm{B}^{-} \rightarrow \mathrm{P}^{+} \mathrm{H}^{-}$. Since the $760 \mathrm{~nm}$ excitation overlaps both $\mathrm{H}_{A}$ and $\mathrm{H}_{B}$, the SADS for this sequence also represent a mixture of the energy transfer kinetics of both branches. 
Global fits of individual excitation bands in DLL only required two compartments each. The SADS are shown in Figure 4.17, and a comparison between the data and SADS fit is shown in Figure 4.18. The 865nm excitation SADS (Figure 4.17A) match the first two SADS from the W(M250)V 865nm excitation (Figure 4.15A), which further supports the conclusion that the SADS derived from P-band excitation represent the actual difference spectra and not linear combinations of them. The SADS from $813 \mathrm{~nm}$ and $793 \mathrm{~nm}$ excitation are similar, with a fast decaying component that is mostly bleaching centered at the excitation wavelength, and a slow-decaying $\mathrm{P}^{*}$ spectrum similar to those in Figure 4.13D and Figure 4.17A. The $\mathrm{P}^{*}$ spectrum also matches that reported in [50].

(A) $865 \mathrm{~nm}$ excitation
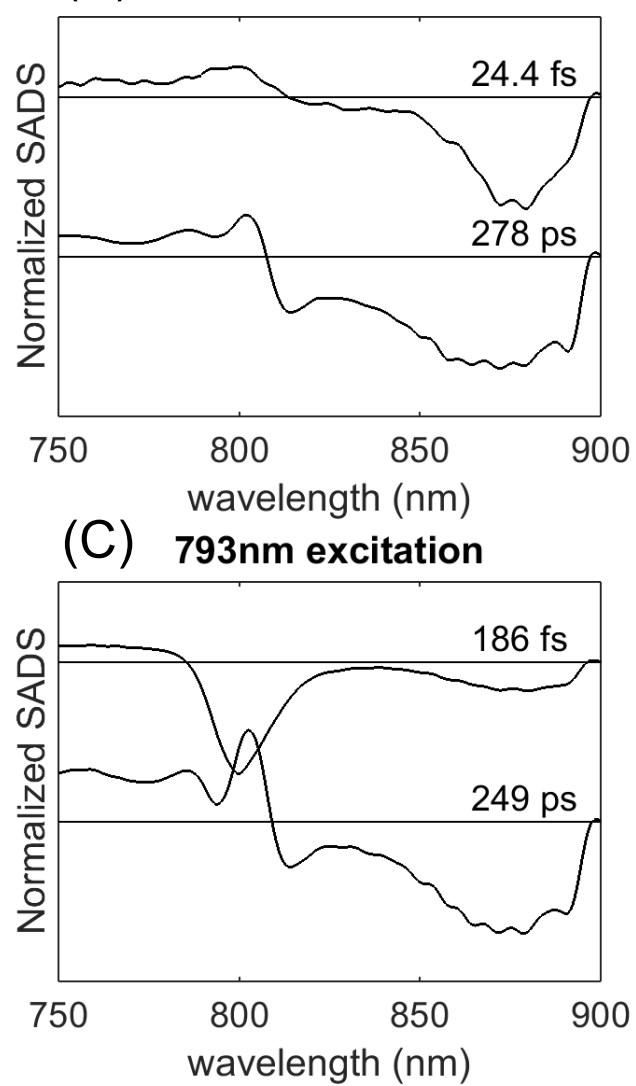

(B) $813 \mathrm{~nm}$ excitation

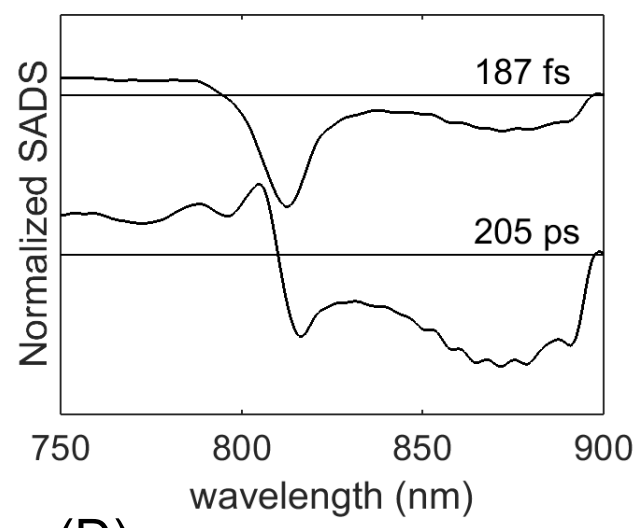

(D) $757 \mathrm{~nm}$ excitation

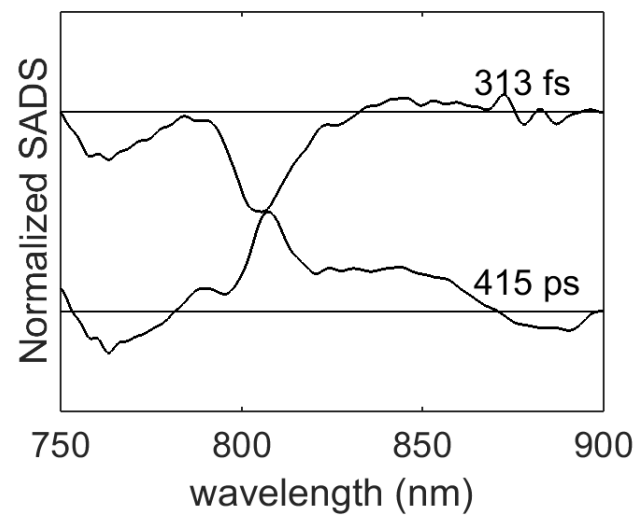

Figure 4.17: 2-compartment SADS calculated from DLL 2D spectra at specific excitation wavelengths.

For DLL, the $757 \mathrm{~nm}$ excitation in Figure 4.17D only excites $\mathrm{H}_{B}$, and the resulting SADS do not match the expected $\mathrm{H}_{B} \rightarrow \mathrm{B}_{B} \rightarrow \mathrm{P}$ energy transfer sequence. The initially excited 
(A) $865 \mathrm{~nm}$ excitation

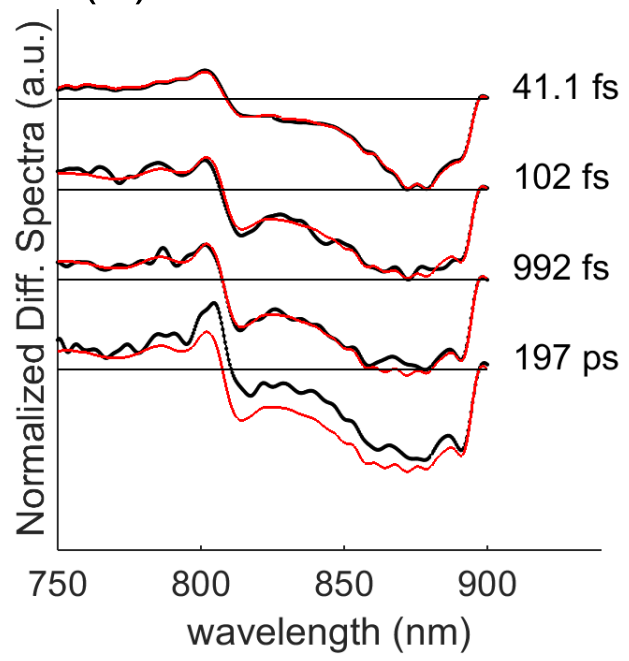

(C) 793nm excitation

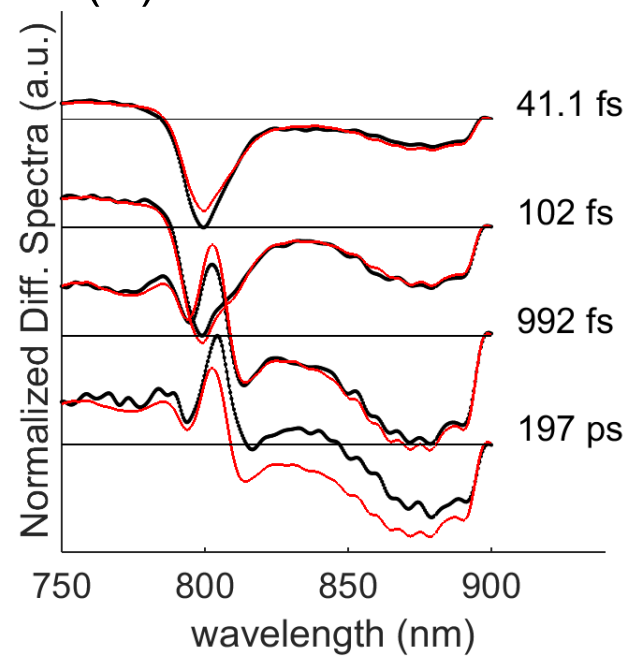

(B) $813 \mathrm{~nm}$ excitation

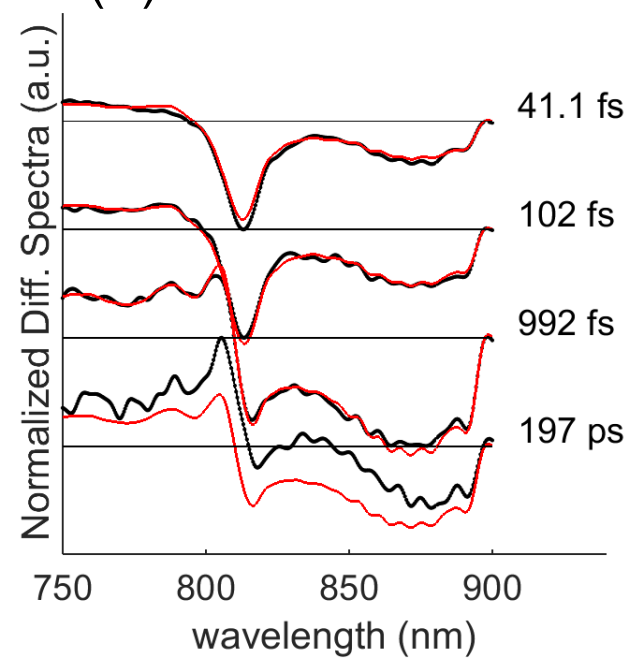

(D) $757 \mathrm{~nm}$ excitation

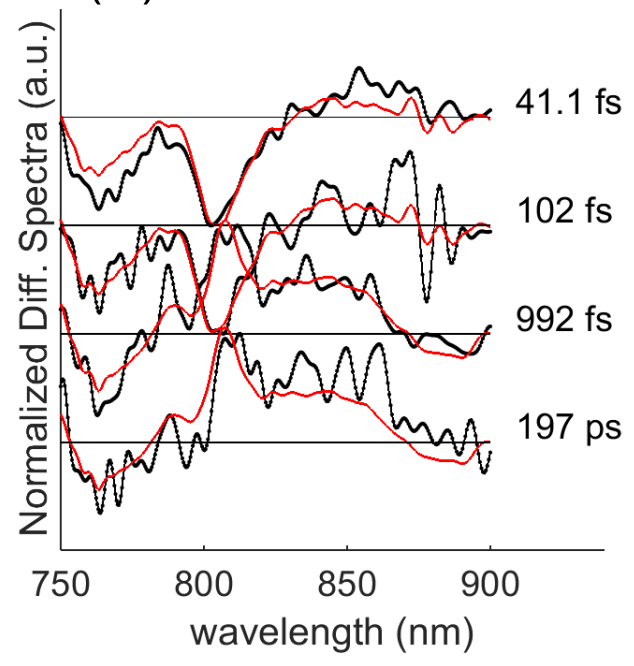

Figure 4.18: Measured spectra (black) and SADS fit (red) from the 2-compartment models shown in Figure 4.17 
state resembles that of Figure 4.16, with simultaneous bleaching of the $\mathrm{H}$ and $\mathrm{B}$ bands. However, unlike the H-band excitation of $\mathrm{W}(\mathrm{M} 250) \mathrm{V}$ which proceeded to $\mathrm{B}^{*}$ with a $55 \mathrm{fs}$ time constant, the DLL H-excitation forms a single product state in 300fs, which decays at a slower rate than the $\mathrm{P}^{*}$ state formed by the other excitation wavelengths. The spectrum associated with this product state does not resemble any state observed in the $\mathrm{W}(\mathrm{M} 250) \mathrm{V}$ SADS. It has a sharp ESA peak at $807 \mathrm{~nm}$, near the P* ESA peaks in Figure 4.17(A-C), but also has a broad pedestal extending to $850 \mathrm{~nm}$ where $\mathrm{P}^{*}$ shows bleaching. The $\mathrm{H}$ band and the $\mathrm{P}$ band at the edge of our detection bandwidth also show bleaching, suggesting $\mathrm{H}_{B}$ and $\mathrm{P}$ are both involved in this state. Examining the raw data and SADS fit in Figure 4.18(D), the H-band signal-to-noise is much lower than the other excitation frequencies, so the fine features of the SADS fit might be misleading. Specifically, the P-band bleaching in the 415ps component may not be real, but the other features of this state are clearly visible from the actual data in both the 1ps and 200ps time traces.

The global fits of the individual excitation bands have revealed a number of common features and notable differences between the kinetic rates and apparent spectra within different subsets of the 2D spectrum, which will be used in the 2D global analysis below to either impose explicit constraints on the model or to check the consistency of the fitting results:

1. The SADS from P-band excitation of W(M250)V and DLL match, and the final product state from $\mathrm{P}$ - and B-band excitation in DLL matches $\mathrm{P}^{*}$ spectra reported in the literature. We can treat this SADS component as the actual difference spectrum of $\mathrm{P}^{*}$.

2. Based on comparison of the $\mathrm{W}(\mathrm{M} 250) \mathrm{V}$ and DLL H-band data, excitations of $\mathrm{H}_{A}$ and $\mathrm{H}_{B}$ result in very different kinetics. H-excitation in DLL forms a different product state than $\mathrm{P}^{*}$, while $\mathrm{W}(\mathrm{M} 250) \mathrm{V}$ H-excitation shows the expected energy transfer to $\mathrm{B}$, followed by formation of $\mathrm{P}^{+} \mathrm{H}_{A}^{-}$. We will look for the presence of this $\mathrm{H}_{B}$ product state in the $2 \mathrm{D}$ global analysis. 
3. The initially excited state in the H-band has bleaching in both the $\mathrm{H}$ and $\mathrm{B}$ bands within 20fs. The initial SADS in Figure 4.17 can also be treated as a pure basis spectrum in the 2D global fit.

4. The inhomogeneity in the B-band is apparent in the rates and SADS from 796nm vs $818 \mathrm{~nm}$ excitation. The fact that the $\mathrm{P}^{+} \mathrm{H}_{A}^{-}$spectrum is different at $818 \mathrm{~nm}$ excitation suggests the formation of different product states depending on whether $\mathrm{B}_{A}$ or $\mathrm{B}_{B}$ is excited. The $2 \mathrm{D}$ fitting should include a model allowing $\mathrm{B}_{A}$ or $\mathrm{B}_{B}$ to terminate at different product states.

\subsection{Multi-excitation Global Analysis}

The global fits of the individual excitation bands treat each slice of the 2D spectrum as an independent measurement, each with a different set of basis spectra and rates. For the excitation-dependent 2D global analysis, the aim is to represent the entire 2D spectrum, at all excitation frequencies, as a sum of a single set of basis spectra with time-dependent concentrations representing the actual mixtures of each state for a given excitation. The excitation-dependent global 2D fitting approach is described in detail in Section 3.2.2.

\subsubsection{Optimization Parameters}

Implementing this approach to test a given kinetic model requires additional information pertaining to the specific system, and parameters defining the model. The following considerations are specific to the implementation of the 2D fitting for the $\mathrm{W}(\mathrm{M} 250) \mathrm{V}$ data:

1. Linear absorption fitting. The initial conditions for the concentrations at each excitation frequency are determined by the relative contributions of each chromophore's absorption to the total linear absorption spectrum. In general, the positions, dipole strengths, and widths of each lineshape are variables in the deconvolution of the linear spectrum. For W(M250)V, we fit the linear spectrum in the 700-900nm spectral region with a sum of six lineshapes corresponding to the Qy absorption bands of the 
four bacteriochlorophyll and two bacteriopheophytin pigments. Due to the strong coupling between the special pair bacteriochlorophylls, there are two excitonically split eigenenergies with associated eigenstates $\mathrm{P}_{+}$and $\mathrm{P}_{-}$. Jordanides et al. [16] calculated the energies and dipole strengths of the excitonic eigenstates for $R$. sphaeroides at 298K based on crystal structure measurements, with coupling terms in the Hamiltonian estimated using the dipole approximation. For the fitting of our $77 \mathrm{~K}$ spectrum, the dipole strengths were fixed at the values given in [16], and the peak positions and widths were allowed to vary to account for the red-shift of the P-band at low temperatures. The results of the linear absorption deconvolution are shown in Figure 4.19, and Table 4.1 gives the associated peak positions and dipole strengths. These values can be optimized simultaneously with the 2D fitting, with the residual being a weighted sum of the residuals from the linear and 2D spectra, but in practice, the results of the optimization were not substantially different between the separate vs simultaneous fits.

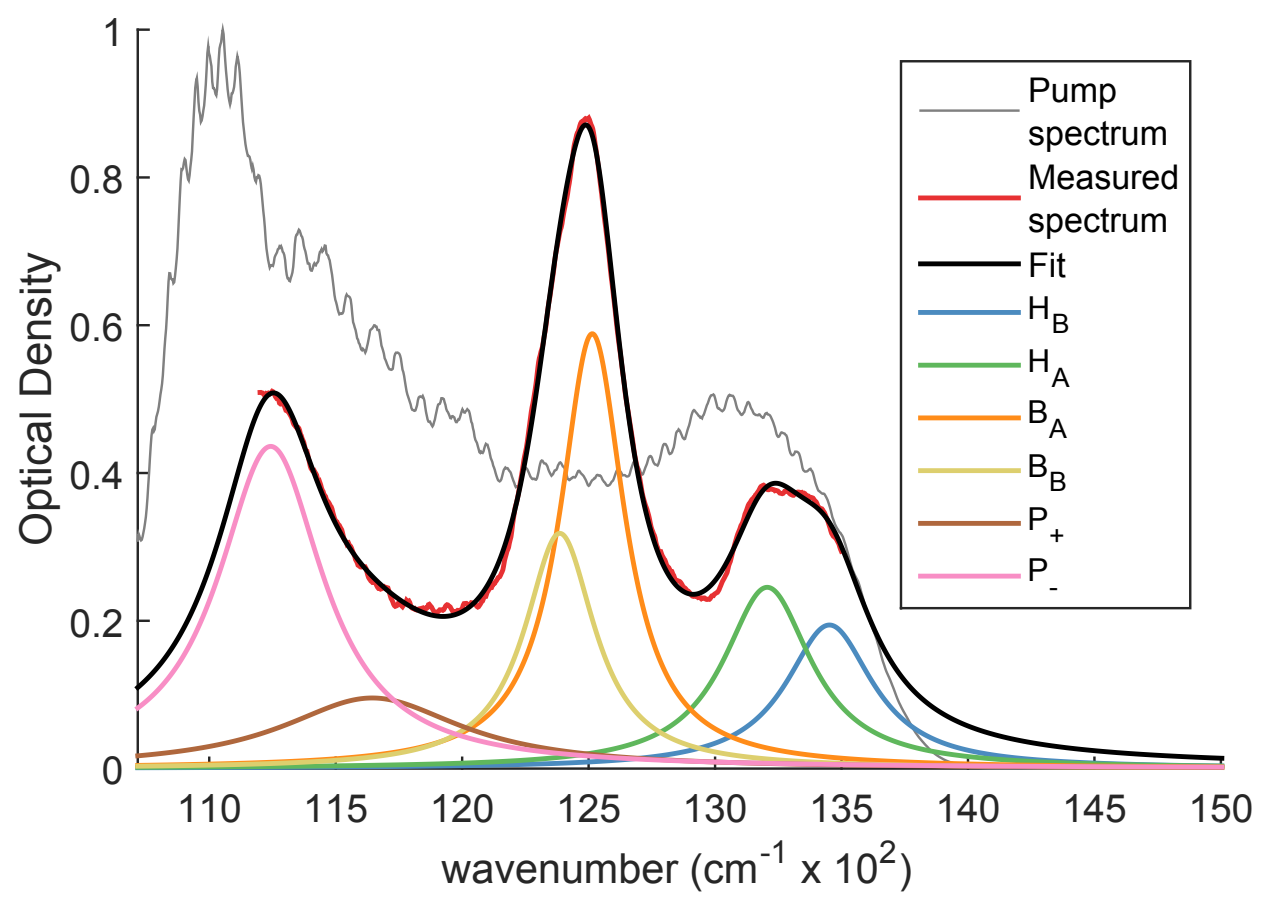

Figure 4.19: 77K linear absorption spectrum of W(M250)V (red) and the resulting fit using six peaks with dipole strengths taken from [16]. 
Table 4.1: Fitting parameters used for W(M250)V 77K linear absorption spectrum

\begin{tabular}{|c|c|c|c|c|c|c|}
\hline Eigenstate & $\mathrm{P}_{-}$ & $\mathrm{P}_{+}$ & $\mathrm{B}_{B}$ & $\mathrm{~B}_{A}$ & $\mathrm{H}_{A}$ & $\mathrm{H}_{B}$ \\
\hline Peak position $\left(\mathrm{cm}^{-1}\right)$ & 11240 & 11640 & 12390 & 12510 & 13210 & 13450 \\
\hline Peak position $(\mathrm{nm})$ & 890 & 859 & 807 & 799 & 757 & 743 \\
\hline Dipole strength $\left(\mathrm{D}^{2}\right)$ & 1.76 & 0.25 & 0.42 & 1.19 & 0.39 & 0.24 \\
\hline
\end{tabular}

2. Defining a kinetic model. As a first step, we must choose the number of states for the model, including the states directly excited by pump absorption, plus the charge separated product states. Generally, there are nonzero rate constants for transitions between all states, constrained by the detailed balance principle such that $k_{j i}=k_{i j} \exp \left(\frac{E_{j}-E_{i}}{k_{B} T}\right)$ where $E_{i}$ and $E_{j}$ are the state energies, $k_{B}$ is the Boltzmann constant, and $T$ is temperature [75]. For the energies in Table 4.1, the up-hill energy transfer is on the order of $10^{-4}$ slower than the down-hill transfer, so back-reactions are assumed to be negligible. Allowing all of the down-hill energy transfer rates to be free parameters in fitting the BRC data proved to be an intractable problem due to the spectral overlap in the B- and H-bands and the similar energy transfer time scales in the two branches, so a more constrained approach to the fitting was necessary.

One way to better-condition the nonlinear optimization of the rates is to constrain the ratios of specific rate constants and use the ratios as nonlinear parameters in the fitting. For example, the $\mathrm{H}_{A} \rightarrow \mathrm{B}_{A}$ and $\mathrm{H}_{B} \rightarrow \mathrm{B}_{B}$ energy transfer rates are expected to be different based on their differing energy gaps and spectral overlap, but they should not be different by orders of magnitude. Allowing the two rates to vary independently can lead to non-physical optimization solutions where one rate is nearly zero, with a basis spectrum that effectively acts as a constant offset for the data fitting rather than an actual state. Constraining one rate to be within a certain range of the other avoids this type of diverging solution. 
3. Constraints on basis spectra. There are several options for how to determine the basis spectra for the 2D fitting. The most straight-forward approach is to solve the linear least squares problem for a given set of rates, which is just the pseudo-inverse solution to Equation 3.20. This is the least constrained option, which effectively gives each state a free linear parameter for every detection frequency. Allowing all of the basis spectra to vary freely can lead to pairs of linearly dependent spectra of opposite sign, like those illustrated in Figure 4.11 from the 2DEAS fitting where the numerically optimal result is not physically reasonable. This is especially problematic for the $\mathrm{B}_{A}$ and $\mathrm{B}_{B}$ spectra.

The second option is to represent the basis spectra as linear combinations of the lineshapes from the linear absorption fit. This approach was employed by Thyrhaug et al. [67] to fit energy transfer dynamics in FMO using 2DES, and further developed by Dostal et al. for general fitting of population transfer from 2DES spectra [75]. Since the linear absorption lineshapes are already constrained from the fit in Figure 4.19, this reduces the number of free linear parameters to $N^{2}$ for an $N$-state model, which can also be solved by linear least squares for each iteration. This approach can help to constrain the $\mathrm{B}_{A}$ and $\mathrm{B}_{B}$ spectra and avoid the nonphysical solutions that arise with the unconstrained least squares spectra. However, the fitting scheme in [75] is not wellsuited to deal with charge transfer states like $\mathrm{P}^{+} \mathrm{H}_{A}^{-}$, which are not included as terms in the linear absorption fitting (they are not directly populated by the excitation pulse), and feature dispersive shapes in their difference spectra from electrochromic shifts $[36,49,61,104]$ which are not well-represented by combinations of the ground state absorption spectra.

Another option is to constrain the basis spectra to a specific, predetermined form, and only perform the nonlinear fitting for the rate constants. Each basis spectrum then has only one unknown positive scaling factor, but this requires some a priori knowledge or physical model for the form of each difference spectrum. If only a subset of the basis spectra are known, this can be combined with the approaches above by using different 
models for the difference spectra of specific species (see Equation 3.25).

4. Optimization method. For fitting the nonlinear variables, we implemented either the trust-region reflective method $[154,155]$ or a particle swarm algorithm [156], using the Matlab Global Optimization Toolbox. The former is well-suited for quickly converging to the minimum near an initial guess of the solution, but can often converge to local minima. The particle swarm method (also used by Thyrhaug et al. for 2D fitting [67]) is better at finding the global minimum by searching the entire parameter space. It is more computationally expensive, but is also easily parallelized.

5. Additional weighting factors. The signal amplitude in the B-band is significantly higher than the $\mathrm{P}$ - and H-bands, so the minimization of the residual will preferentially optimize the fit to the B-band excitation at the expense of the other excitation frequencies. The point of the $2 \mathrm{D}$ global fitting is to find a kinetic model consistent with data from all excitation conditions, so a constant weighting factor is applied to the residuals from each excitation frequency, normalized to the amplitude of the peak of the $\mathrm{P}^{+} \mathrm{H}_{A}^{-}$ spectrum at $\mathrm{T}=1 \mathrm{~ns}$.

We also found it necessary to add an additional excitation-dependent scaling factor to the fitted spectrum to get good agreement with the data. Essentially, this amounts to adjusting the excitation energy, rescaling the time-dependent concentrations for each $\lambda_{e x}$ while retaining the same ratios of concentrations. There are a number of factors that could explain the need for this scaling factor. Phase-mismatch and signal reabsorption effects can cause distortions to 2D spectra of samples with high optical density [157]. With the broad 200nm DOPA bandwidth, the spatial chirp of the laser pulses can cause spatially-dependent frequency overlap between the pump and probe which might also attenuate the $2 \mathrm{D}$ spectrum near the edges of the bandwidth. While we did correct for the frequency-dependent quantum efficiency of the spectrometer CCD, the diffraction efficiency of the spectrometer grating and reflectance of the optics 
between the sample and the spectrometer could also account for a discrepancy in the measured pump spectrum, which was used to calculate the excitation probabilities for the fitting. This scaling factor consistently rescales the reconstructed signal to be lower than expected at the edges of the spectrum, which is consistent with the idea that the measured 2D spectrum is slightly attenuated due to pump/probe overlap effects.

\subsubsection{Kinetic model \#1}

The first reaction scheme to test against the W(M250)V 2DES data is the straightforward downhill energy transfer $\mathrm{H} \rightarrow \mathrm{B} \rightarrow \mathrm{P}$ on the A- and B- branches, followed by the charge separation sequence $\mathrm{P}^{*} \rightarrow \mathrm{P}^{+} \mathrm{B}_{A}^{-} \rightarrow \mathrm{P}^{+} \mathrm{H}_{A}^{-}$. This model can be represented by seven time constants defined in Figure 4.20, where the rate constants $k_{i j}=1 / \tau_{i j}$. The indices 1-6 correspond to the states in Table 4.1 in order of decreasing energy, and indices 7-8 are the charge-separated states. The pairs of time constants $\tau_{14} / \tau_{23}$ and $\tau_{35} / \tau_{45}$ were constrained to ratios between 0.5 and 2, for the reasons discussed in point (2) above.

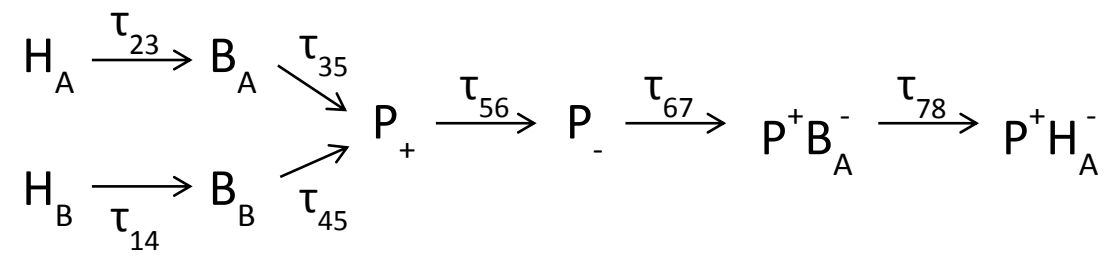

Figure 4.20: Reaction scheme of model \#1 used for 2D multi-excitation global fitting

For the choice of basis spectra, several were constrained based on the results from Section 4.3. Since we concluded that the $865 \mathrm{~nm}$-excitation SADS from the DLL global fit in Figure 4.17 must correspond to $P_{+}$and $P_{-}$difference spectra, which also match the $36 \mathrm{fs}$ and 2.1ps components from the $\mathrm{W}(\mathrm{M} 250) \mathrm{V}$ 865nm-excitation SADS (Figure 4.13D), we used those as the $P_{+}$and $P_{-}$basis spectra in the multi-excitation fit. The fastest-decaying compartment of the H-band excitation in Figure 4.16, which is almost entirely from excitation of $\mathrm{H}_{A}$ and $\mathrm{H}_{B}$, was defined as the basis spectrum for H-excitation in the global fit. In terms of Equation 3.25, the constrained spectra listed above are $\boldsymbol{F}_{1}=\boldsymbol{G} \boldsymbol{L}$, where $\boldsymbol{G}=\operatorname{diag}\left(g_{1}, \ldots, g_{4}\right)$ 
are scaling factors, and the rows of $\boldsymbol{L}$ are the corresponding SADS from Section 4.3. The remaining three unconstrained basis spectra comprise $\boldsymbol{F}_{2}$.

Under the assumptions described above, we ran the nonlinear optimization using a variety of initial conditions for the rate constants near the values obtained from the individual global fits in Section 4.3. The fitting parameters were the seven rate constants, scaling factors for the four constrained basis spectra, and the excitation-dependent weighting function discussed above. Note that the gradient-based optimization will only converge to the same solution within a certain radius in the parameter space. Even with the additional constraints imposed by the multi-excitation fitting, there can still be found pairs of solutions with nearly identical residuals. For example, interchanging the time constants $\tau_{67}$ and $\tau_{78}$ results in a significantly different least-squares spectrum for $\mathrm{P}^{+} \mathrm{B}_{A}^{-}$but negligible difference in residual, which is related to the same uniqueness problem of sequential models discussed in Section 3.1.2.

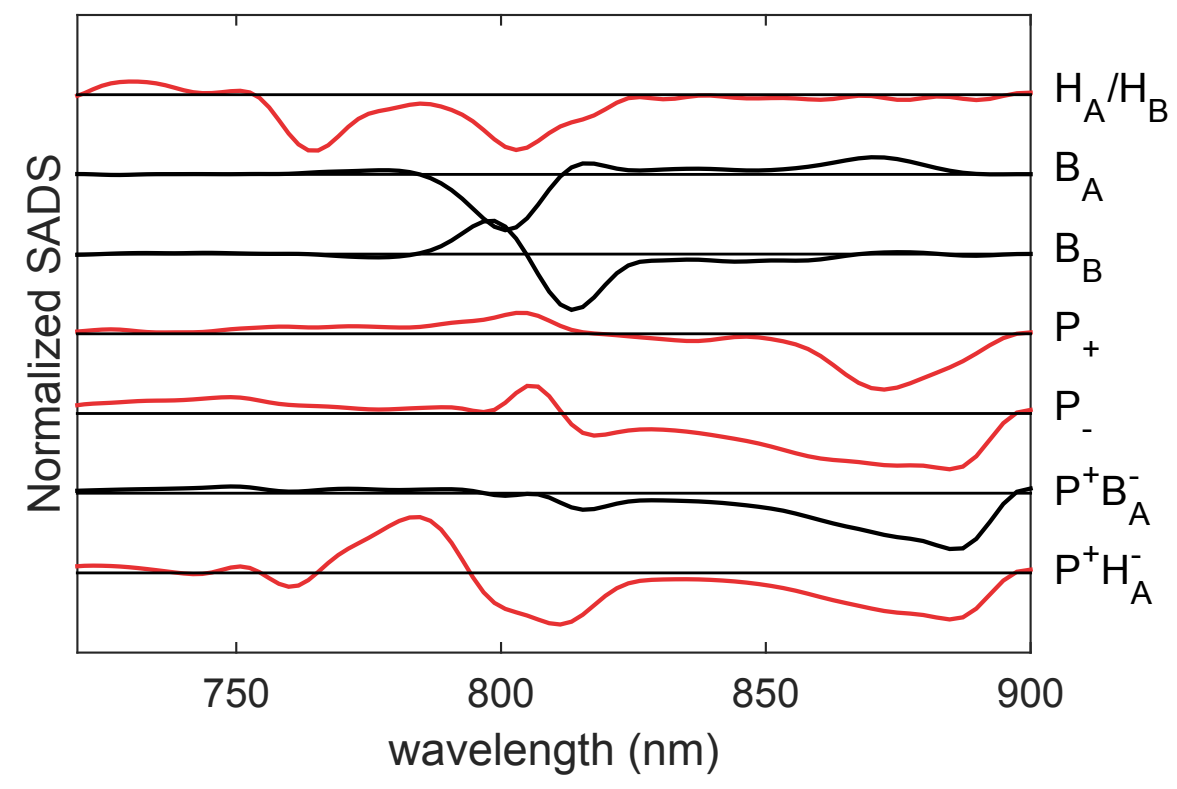

Figure 4.21: Basis spectra from the global analysis of W(M250)V. The spectra plotted in red were constrained to match the SADS from Figure 4.13D-E. The black basis spectra were calculated by linear least squares.

The optimization for this model yielded the rate constants in Table 4.2 with associated basis spectra in Figure 4.22. The energy transfer rates from $\mathrm{H}$ match the single-band fitting 
Table 4.2: Optimized time constants for the kinetic model depicted in Figure 4.20

\begin{tabular}{|c|c|c|}
\hline Time constant & Transition & Optimized value \\
\hline$\tau_{14}$ & $\mathrm{H}_{B} \rightarrow \mathrm{B}_{B}$ & $55 \mathrm{fs}$ \\
\hline$\tau_{23}$ & $\mathrm{H}_{A} \rightarrow \mathrm{B}_{A}$ & $52 \mathrm{fs}$ \\
\hline$\tau_{35}$ & $\mathrm{~B}_{A} \rightarrow \mathrm{P}_{+}$ & $197 \mathrm{fs}$ \\
\hline$\tau_{45}$ & $\mathrm{~B}_{B} \rightarrow \mathrm{P}_{+}$ & $169 \mathrm{fs}$ \\
\hline$\tau_{56}$ & $\mathrm{P}_{+} \rightarrow \mathrm{P}_{-}$ & $36 \mathrm{fs}$ \\
\hline$\tau_{67}$ & $\mathrm{P}_{-} \rightarrow \mathrm{P}^{+} \mathrm{B}_{A}^{-}$ & $490 \mathrm{fs}$ \\
\hline$\tau_{78}$ & $\mathrm{P}^{+} \mathrm{B}_{A}^{-} \rightarrow \mathrm{P}^{+} \mathrm{H}_{A}^{-}$ & $2.1 \mathrm{ps}$ \\
\hline
\end{tabular}

case almost exactly, since we used the same basis spectrum and the $\mathrm{H} \rightarrow \mathrm{B}$ reaction is unaffected by changes to the other rate constants. The $\mathrm{B} \rightarrow \mathrm{P}$ times are close to the 160fs reported by Stanley et al. [33] and King et al. [32]. The 36fs $\mathrm{P}_{+} \rightarrow \mathrm{P}_{-}$internal conversion is faster than the 50-100fs observed by Vos et al. [54], which might be attributed to the improved time resolution of the 2DES measurement over the 30fs instrument response reported in their study. The $0.49 \mathrm{ps}$ component is significantly faster than the often-cited $0.9 \mathrm{ps} \mathrm{P}^{*} \rightarrow \mathrm{P}^{+} \mathrm{B}_{A}^{-}$rate, while the total time for the $\mathrm{P}^{*} \rightarrow \mathrm{P}^{+} \mathrm{H}_{A}^{-}$transition is reasonably close to the accepted $2.8 \pm 0.2 \mathrm{ps}$ time scale first reported by [158]. In fact, our measurements are not inconsistent with that rate; fixing the rate constants to those values still yields a reasonable fit to the $2 \mathrm{D}$ spectra, so long as the sum of $\tau_{67}$ and $\tau_{78}$ are near the optimal 2.6ps. This point will be discussed further in Section 4.5.

Figure 4.22 and Figure 4.23 compare the measured 2D spectra to the spectra reconstructed from the global analysis. The fit retains most of the features of the measured spectra, including the positions, amplitudes and widths of the cross-peaks, the splitting of the B-band diagonal peaks at 350fs, and the structure of the above-diagonal ESA. Crosssection comparisons of the data and global fit in Figure 4.24 show very good agreement across the entire spectrum from 85 fs to $45 \mathrm{ps}$. The one region where the global fit does a poor job of reproducing the data is the B-band diagonal peak after $\approx 1$ ps. The measured 2D spectrum retains an inhomogeneous line shape along the diagonal which persists for the 

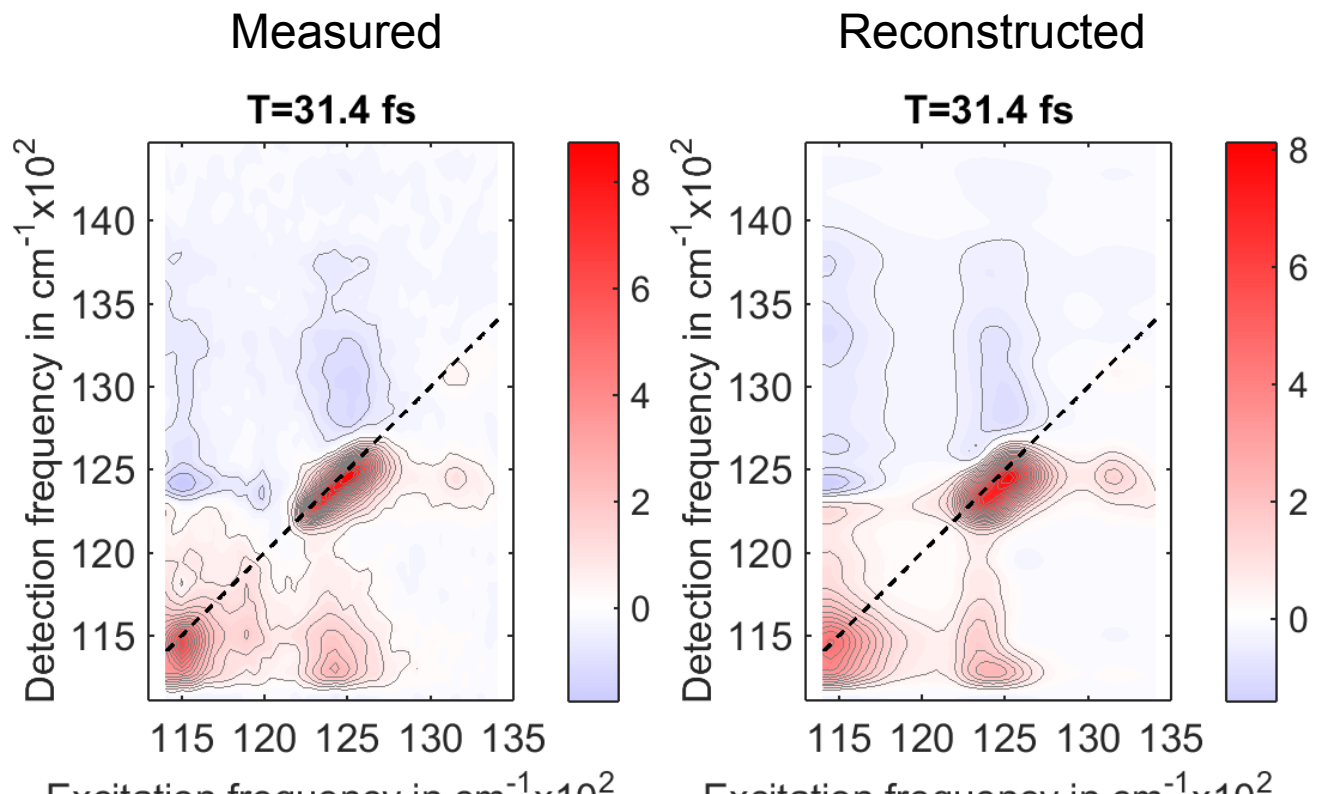

Excitation frequency in $\mathrm{cm}^{-1} \times 10^{2}$

Excitation frequency in $\mathrm{cm}^{-1} \times 10^{2}$
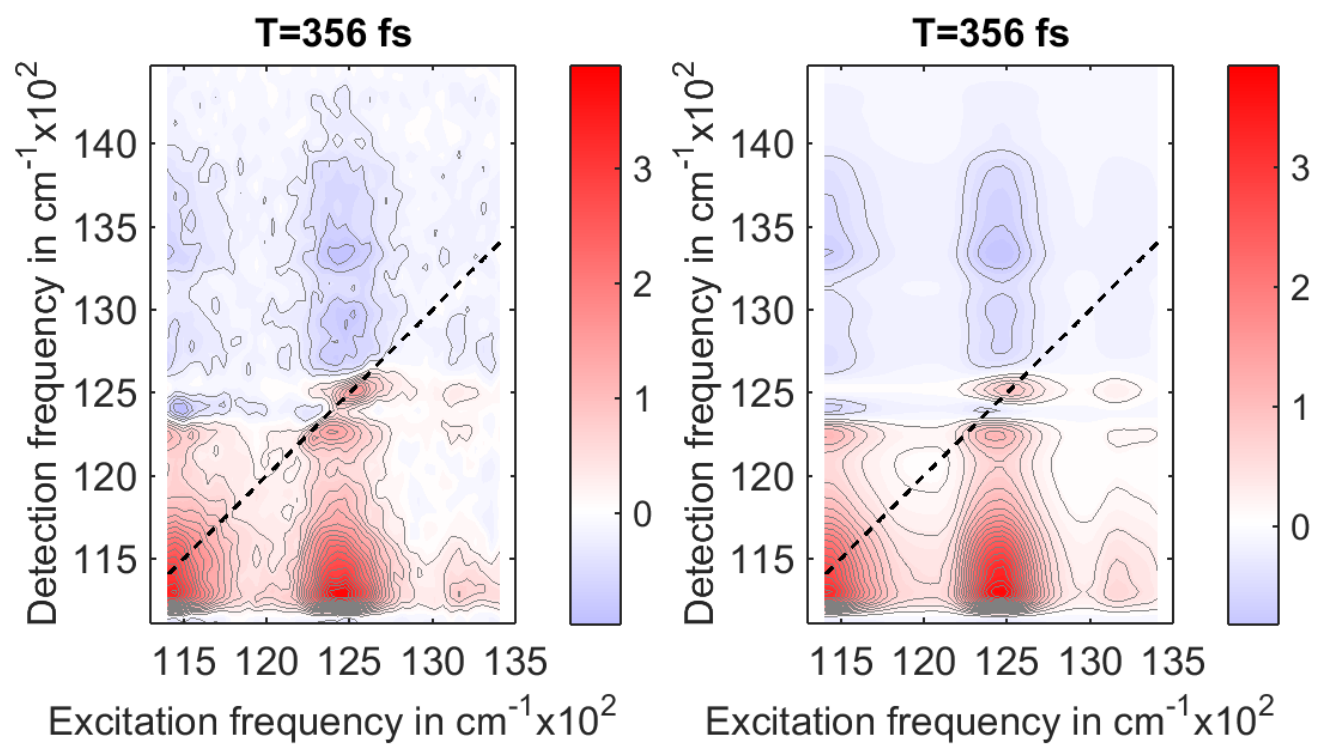

Figure 4.22: Comparison of measured 2D spectra (left) and 2D spectra reconstructed from the global fit (right) at population times of 31.4fs (top) and 356fs (bottom) 

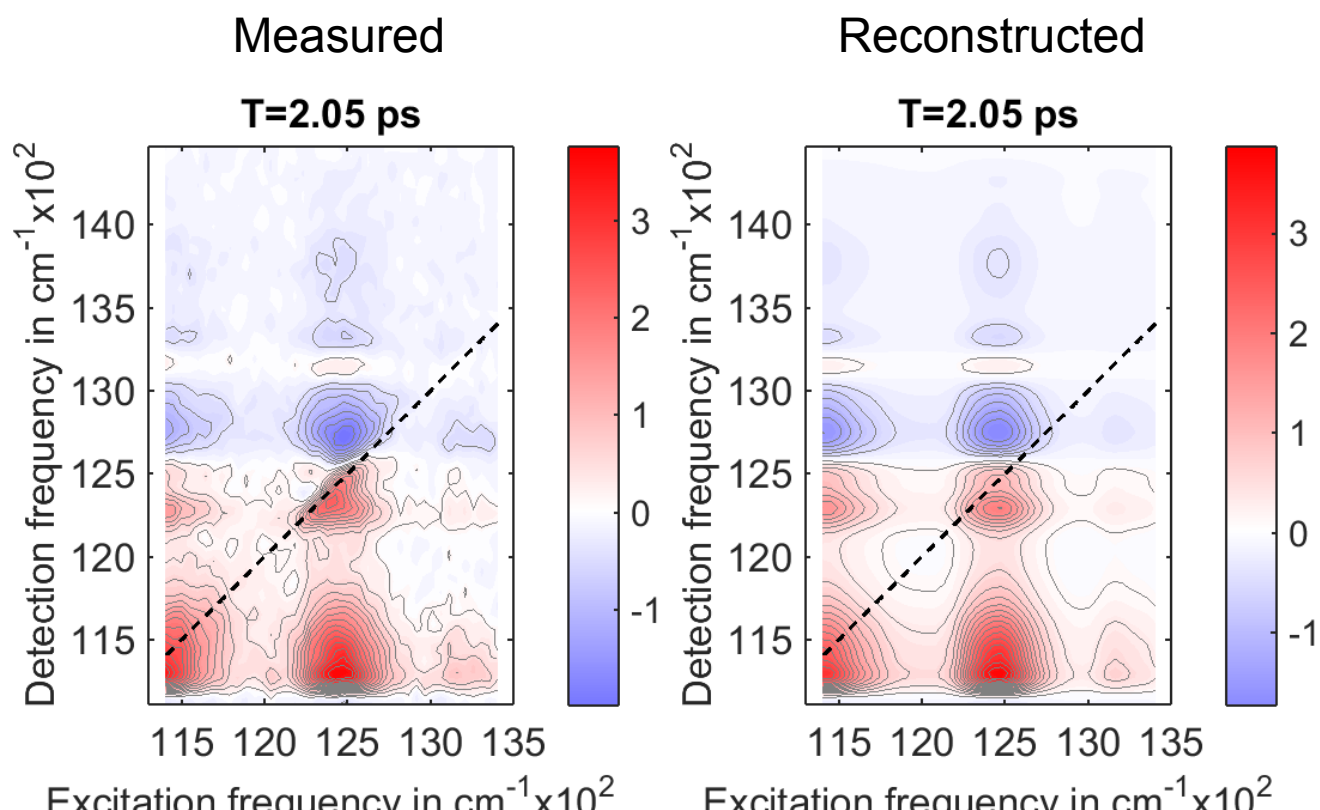

Excitation frequency in $\mathrm{cm}^{-1} \times 10^{2}$
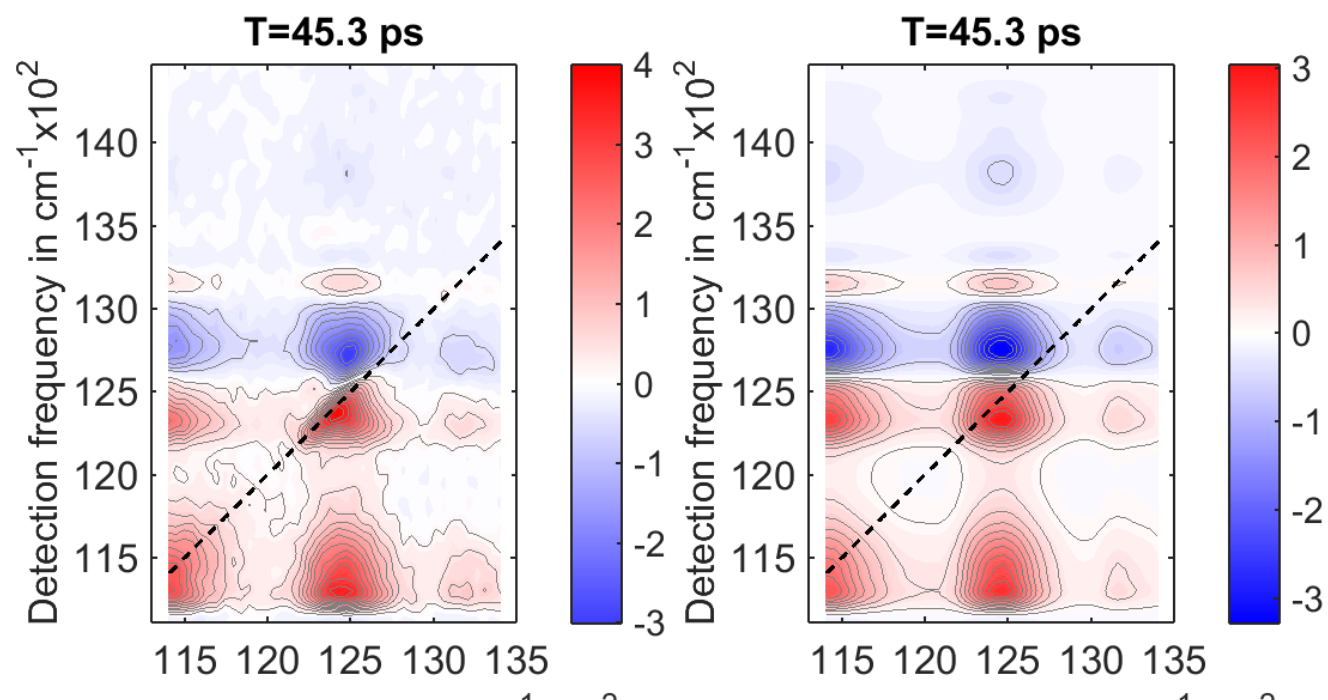

Excitation frequency in $\mathrm{cm}^{-1} \times 10^{2} \quad$ Excitation frequency in $\mathrm{cm}^{-1} \times 10^{2}$

Figure 4.23: Comparison of measured 2D spectra (left) and 2D spectra reconstructed from the global fit (right) at population times of 2ps (top) and 45ps (bottom) 
entire 1ns scan. The expectation with the reaction scheme above is that the detected spectrum should become excitation-independent at population times long enough for $\mathrm{P}^{+} \mathrm{H}_{A}^{-}$to have formed. The persisting inhomogeneous peak might indicate that some fraction of the photo-excited bacteriochlorophylls do not undergo energy transfer. It is also notable that the $S_{z}$ component of the polarization associated spectrum in Figure 4.4 exhibits much more of this inhomogeneity than the $S_{y}$ component, with excitation frequency cross-sections that resemble derivative line shapes.

Examining the features in the optimized SADS for this model in Figure 4.21, the $\mathrm{B}_{A}$ and $\mathrm{B}_{B}$ peak positions are near the positions we expect based on the linear absorption fit, but the positive (i.e. increased absorption) peak in the $\mathrm{B}_{B}$ spectrum is unexpected. At early population times after a B-band excitation excites a mixture of $\mathrm{B}_{A}$ and $\mathrm{B}_{B}$, the positive $\mathrm{B}_{B}$ peak cancels the $\mathrm{B}_{A} \mathrm{SE}$ peak, which allows the global fit to approximate the inhomogeneous broadening in Figure 4.22. The shape of the $\mathrm{B}_{B}$ spectrum in Figure 4.21 is likely nonphysical, and a more sophisticated model is required to properly account for the B-band inhomogeneity.
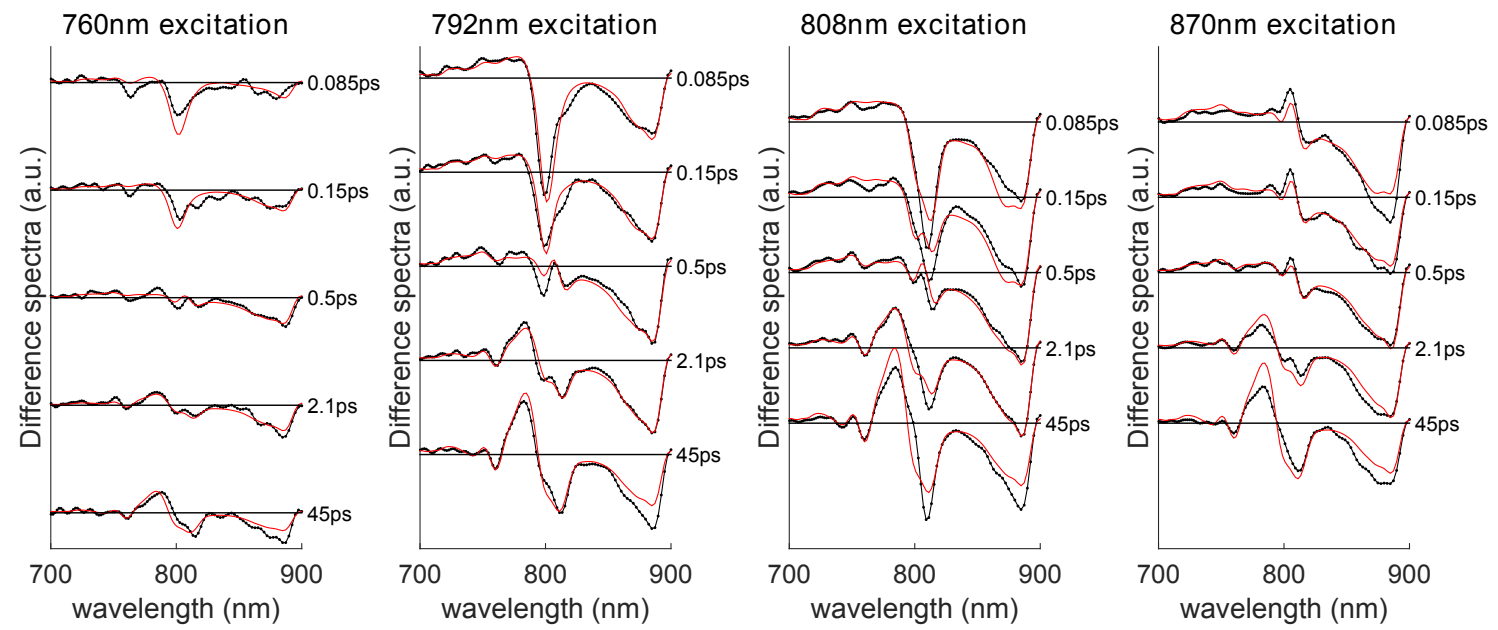

Figure 4.24: Comparison between W(M250)V 2DES data (black) and reconstructed data from global fit (red) at several excitation wavelengths and population times.

To test this interpretation of the $\mathrm{B}_{B}$ basis spectrum, we tried fitting the same kinetic model while also constraining the $\mathrm{B}_{A}$ and $\mathrm{B}_{B}$ spectra to match the initial SADS in Fig- 

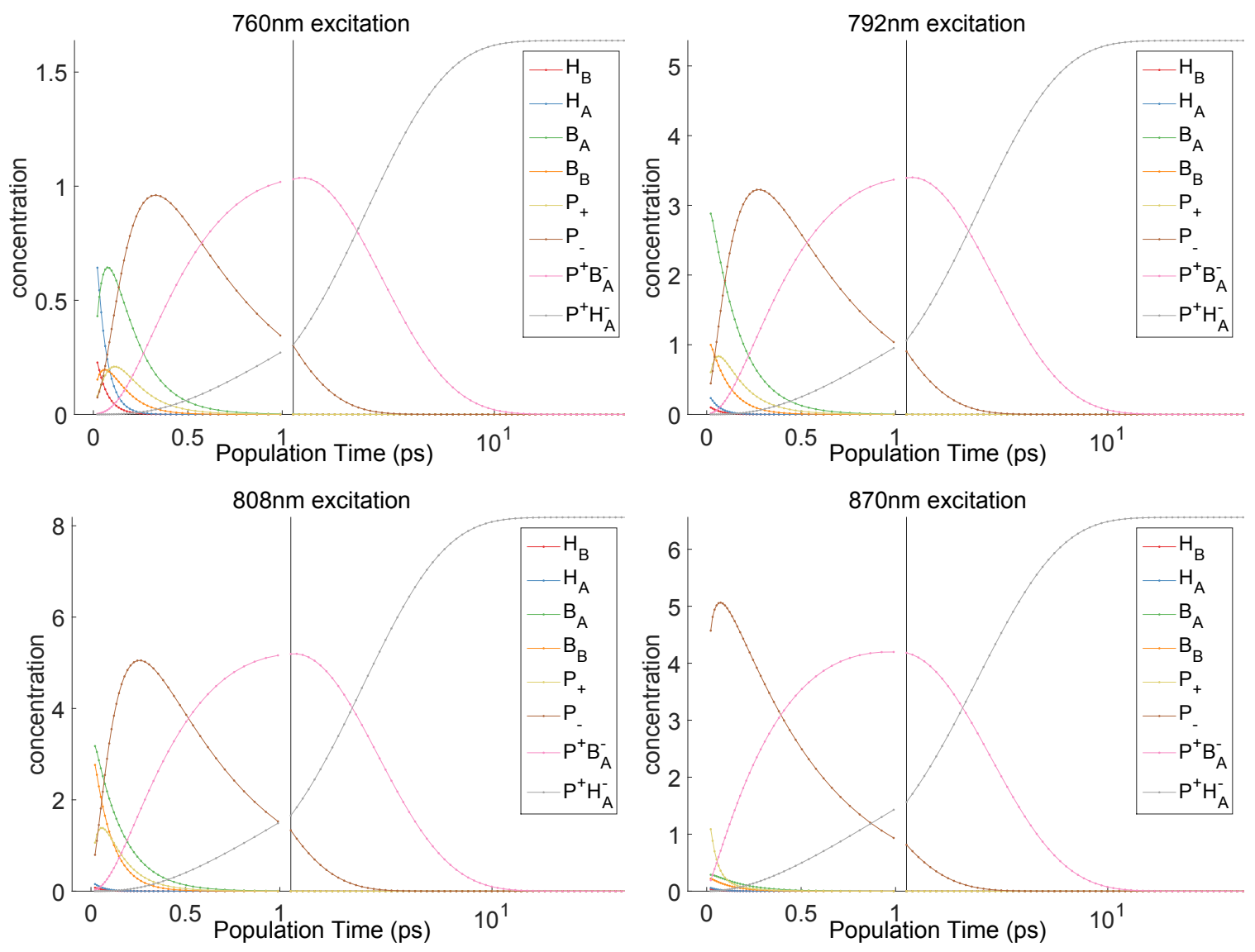

Figure 4.25: Time-dependent concentrations from global fit at several excitation wavelengths representing selective excitation of different mixtures of chromophores in the $\mathrm{P}, \mathrm{B}$ and $\mathrm{H}$ absorption bands. 

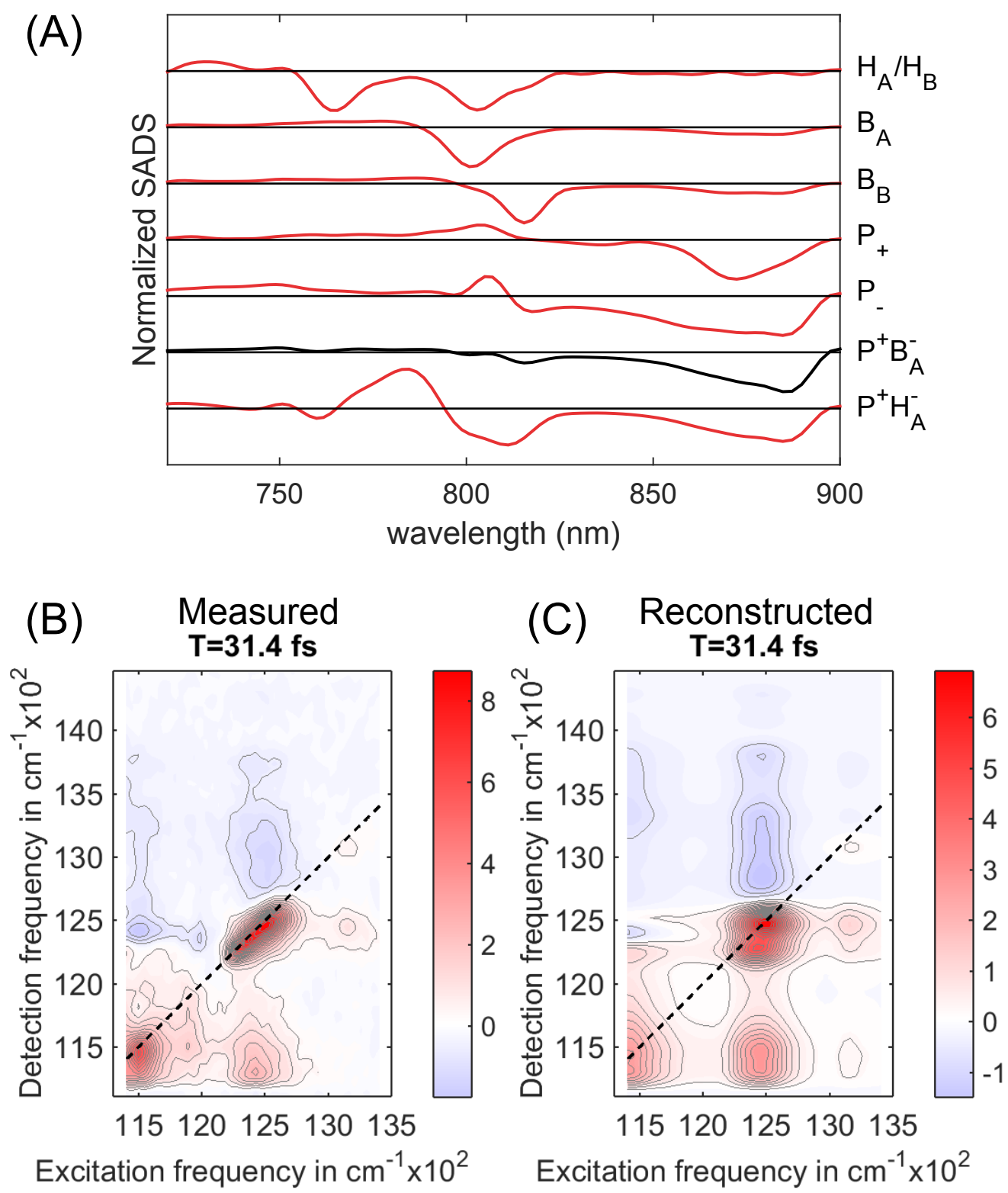

Figure 4.26: (A) Basis spectra from 2D global fit with the reaction scheme in Figure 4.20. Red spectra were constrained to match the SADS from Figure 4.13D-E, and the black spectrum was calculated by linear least squares. (B) Measured 2D spectrum at 31fs. (C) $2 \mathrm{D}$ spectrum reconstructed using the basis spectra from $(\mathrm{A})$. 
ure 4.13. The resulting fit, shown in Figure 4.26 at $31 \mathrm{fs}$, does not reproduce the measured homogeneous linewidth (i.e. the anti-diagonal cross-section) of the B-band signal nearly as well as the first set of basis spectra. Dostal et al. noted this as a limitation in their 2D fitting method [75], which is essentially equivalent to the way the $\mathrm{B}_{A}$ and $\mathrm{B}_{B}$ spectra are defined for Figure 4.26.

\subsubsection{Kinetic model \#2}

For the second kinetic model, we look for evidence of a branched charge separation reaction from $\mathrm{B}_{A}$ proposed in several studies $[51-53,57]$. Figure $4.27 \mathrm{~A}$ shows the reaction scheme for this model, now involving nine spectra with the addition of a $\mathrm{B}_{A}^{+} \mathrm{H}_{A}^{-}$intermediate state, and nine rate constants. For the branched reaction from $\mathrm{B}_{A}$, the rate constants $\tau_{35}$ and $\tau_{37}$ are related by a nonlinear parameter defining the ratio of $\mathrm{P}^{*}$ and $\mathrm{B}_{A}^{+} \mathrm{H}_{A}^{-}$yields. The basis spectra associated with $\mathrm{H}, \mathrm{P}_{+}, \mathrm{P}_{-}$and $\mathrm{P}^{+} \mathrm{H}_{A}^{-}$are constrained in the same way as the first model. The resulting spectra after optimization are shown in Figure 4.27B, along with cross-sections of the data and fit in Figure $4.27 \mathrm{C}$ to illustrate the improvement in residual compared to Figure 4.24. The optimized rate constants are in Table 4.3. The initial conditions for the optimization were the optimized rates from Table 4.2, with $\tau_{37}$ and $\tau_{35}$ initially assumed to be equal.

Table 4.3: Optimized time constants for the second kinetic model, depicted in Figure 4.27

\begin{tabular}{|c|c|c|}
\hline Time constant & Transition & Optimized value \\
\hline$\tau_{14}$ & $\mathrm{H}_{B} \rightarrow \mathrm{B}_{B}$ & $169 \mathrm{fs}$ \\
\hline$\tau_{23}$ & $\mathrm{H}_{A} \rightarrow \mathrm{B}_{A}$ & $161 \mathrm{fs}$ \\
\hline$\tau_{35}$ & $\mathrm{~B}_{A} \rightarrow \mathrm{P}_{+}$ & $293 \mathrm{fs}$ \\
\hline$\tau_{37}$ & $\mathrm{~B}_{B} \rightarrow \mathrm{B}_{A}^{+} \mathrm{H}_{A}^{-}$ & $224 \mathrm{fs}$ \\
\hline$\tau_{45}$ & $\mathrm{~B}_{B} \rightarrow \mathrm{P}_{+}$ & $146 \mathrm{fs}$ \\
\hline$\tau_{56}$ & $\mathrm{P}_{+} \rightarrow \mathrm{P}_{-}$ & $36 \mathrm{fs}$ \\
\hline$\tau_{68}$ & $\mathrm{P}_{-} \rightarrow \mathrm{P}^{+} \mathrm{B}_{A}^{-}$ & $1.68 \mathrm{ps}$ \\
\hline$\tau_{79}$ & $\mathrm{~B}_{A}^{+} \mathrm{H}_{A}^{-} \rightarrow \mathrm{P}^{+} \mathrm{H}_{A}^{-}$ & $1.99 \mathrm{ps}$ \\
\hline$\tau_{89}$ & $\mathrm{P}^{+} \mathrm{B}_{A}^{-} \rightarrow \mathrm{P}^{+} \mathrm{H}_{A}^{-}$ & $243 \mathrm{ps}$ \\
\hline
\end{tabular}


(A)

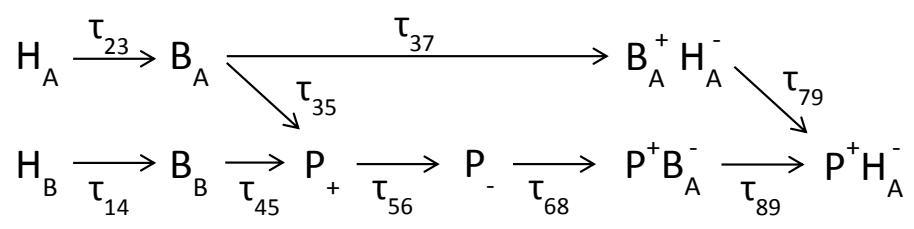

(B)
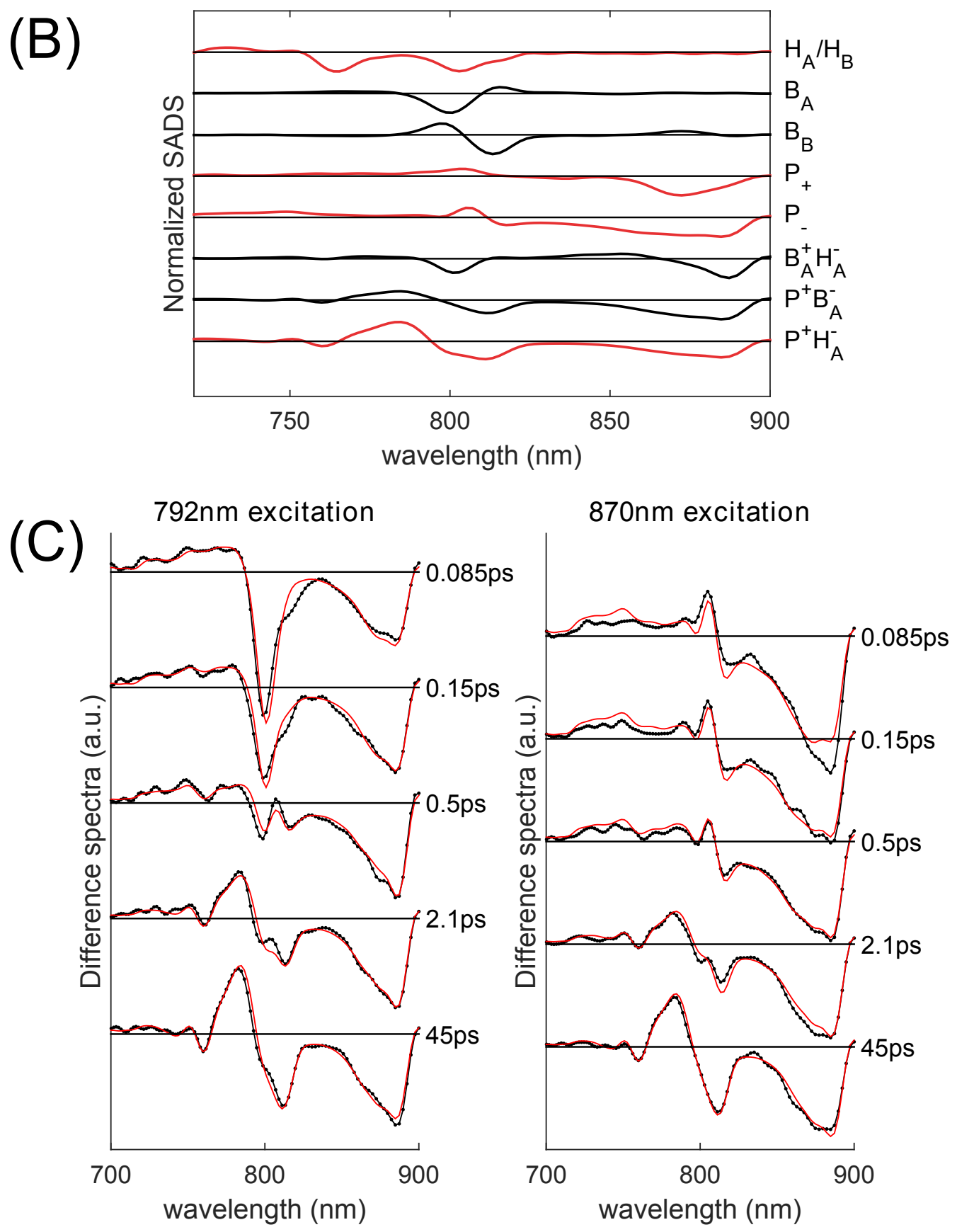

Figure 4.27: (A) 9-state branched reaction scheme defining kinetic model \#2. (B) Optimized basis spectra, with red/black being constrained/unconstrained as in model 1. (C) Measured spectra (black) vs fit (red) at several population times and excitation wavelengths. 
The $\mathrm{B}_{A}$ and $\mathrm{B}_{B}$ spectra for this model share the same mutually canceling peaks that we saw in the first model, due to the same problem fitting the inhomogeneous line shape of the B-band. The charge separation timescales from this model are inconsistent with the dynamics observed directly from the data, and from the individual SADS fits in Section 4.3, with an very slow $243 \mathrm{ps}^{+} \mathrm{B}_{A}^{-} \rightarrow \mathrm{P}^{+} \mathrm{H}_{A}^{-}$rate, while the $\mathrm{B}_{A}^{+} \mathrm{H}_{A}^{-}$pathway takes on the $2 \mathrm{ps}$ dynamics. The $\mathrm{B}_{A}^{+} \mathrm{H}_{A}^{-}$spectrum resembles the $\mathrm{P}^{+} \mathrm{H}_{A}^{-}$spectrum from model 1, while the slow $\mathrm{P}^{+} \mathrm{B}_{A}^{-}$spectrum is nearly identical to that of $\mathrm{P}^{+} \mathrm{H}_{A}^{-}$. The time scale and spectral features of the $\mathrm{B}_{A}^{+} \mathrm{H}_{A}^{-}$spectrum suggest that, rather than being the signature of the proposed branched $\mathrm{B}_{A}^{*} \rightarrow \mathrm{B}_{A}^{+} \mathrm{H}_{A}^{-}$pathway, the optimization algorithm simply converges to a solution in which the $\mathrm{B}_{A}^{+} \mathrm{H}_{A}^{-}$compartment takes on the role that $\mathrm{P}^{+} \mathrm{B}_{A}^{-}$played in model 1 to replicate the 2ps dynamics, while the new $\mathrm{P}^{+} \mathrm{B}_{A}^{-}$basis spectrum in Figure 4.27 is used to make small adjustments to the shape of the $\mathrm{P}^{+} \mathrm{H}_{A}^{-}$spectrum. Only the first 50ps of the measured 2D data were used for these fits, so the $\tau_{89}=243$ ps rate is essentially a constant.

The results of Figure 4.27 highlight the inherent challenges of uniquely identifying branched reactions from transient spectra. In this case, even with half of the basis spectra constrained to be reasonable approximations to the real species associated spectra, exchanging the values of $\tau_{79}$ and $\tau_{89}$ results in global fits with nearly identical residuals and exchanged $\mathrm{P}^{+} \mathrm{B}_{A}^{-}$and $\mathrm{B}_{A}^{+} \mathrm{H}_{A}^{-}$basis spectra. This model and other branched models such as $\mathrm{B}^{*} \rightarrow \mathrm{P}^{+} \mathrm{B}_{A}^{-}$do not converge to yield physically reasonable basis spectra, and applying different ratios between time constants for the two branches yields fits with negligible difference in residual. Based on the results of the nonlinear fitting for the model in Figure 4.27A and a number of other branched reaction schemes, we conclude that the information contained in the 2DES spectrum does not reveal compelling evidence of direct charge separation upon photo-excitation of $\mathrm{B}_{A}$, either by way of $\mathrm{B}^{*} \rightarrow \mathrm{P}^{+} \mathrm{B}_{A}^{-}$or $\mathrm{B}^{*} \rightarrow \mathrm{B}_{A}^{+} \mathrm{H}_{A}^{-}$. Since including additional charge separation intermediates does not produce a clear improvement in the residual with physically reasonable basis spectra, the most reasonable conclusion is that our data supports the simpler kinetic model \#1. 
The fitting results with the branched reaction models do not disprove the existence of these parallel charge separation pathways. Many such models are consistent with the 2D data, so quantitative assignment of the relative yields for each pathway is not yet possible. This suggests that the information extracted from global analysis of transient absorption data is also insufficient to unambiguously assign specific branched kinetic schemes. The 2D fitting approach we employed essentially takes the information from dozens of transient absorption experiments at different excitation frequencies, models the relationship between them based on the absorption spectrum and crystal structure measurements, and attempts to discriminate between kinetic models. Therefore, distinguishing between different kinetic models from transient absorption experiments alone can only be more difficult than when using the richer data set available from 2DES.

\subsection{Discussion}

In this chapter, we have presented broadband polarization-dependent 2D spectra of two BRC mutants from $R$. capsulatus, on timescales ranging from $10 \mathrm{fs}$ to $1 \mathrm{~ns}$. This multidimensional dataset contains a wealth of information on energy and charge transfer processes, with time and excitation frequency resolutions not achievable with transient absorption spectroscopy. We applied several global and target analysis techniques to disentangle the reaction kinetics and extract physically meaningful rate constants and spectral signatures. 2DDAS and 2DEAS provided estimates of the rate constants and qualitative information revealing which regions of the spectrum change on particular timescales. Global fits on subsets of the 2D spectra at specific excitation frequencies proved useful for identifying the difference spectra of $\mathrm{P}_{+}, \mathrm{P}_{-}, \mathrm{P}^{+} \mathrm{H}_{A}^{-}$. Global analysis of the $\mathrm{H}$ excitation band revealed that signficant $\mathrm{H} \rightarrow \mathrm{B}$ energy transfer occurs within the first $20 \mathrm{fs}$, which is faster than previously observed $[54,153]$. We also observe $\mathrm{P}_{+} \rightarrow \mathrm{P}_{-}$energy transfer with a $36 \mathrm{fs}$ time constant that is faster than previous estimates [54], and clearly resolve the homogeneous and inhomogeneous widths of the B-band. 
Using our multi-excitation 2D global fitting approach, we find a minimal representation of the 2D spectrum using a common set of basis spectra and rate constants for all excitation frequencies. Using the entire 2D spectrum, the linear absorption spectrum, additional constraints from direct comparison of the kinetics of the two mutants, and dipole strengths calculated from the crystal structure, we can reproduce the 2D spectrum with eight species associated spectra and eight rate constants, using a model with only the $\mathrm{P}^{*} \rightarrow \mathrm{P}^{+} \mathrm{B}_{A}^{-} \rightarrow \mathrm{P}^{+} \mathrm{H}_{A}^{-}$ charge separation pathway. This method reduces the number of free parameters by an order of magnitude compared to 2DEAS, and imposes a physical model of the interdependence of different columns of the $2 \mathrm{D}$ spectrum. With the exception of $\mathrm{B}_{A}$ and $\mathrm{B}_{B}$, the basis spectra correspond to the actual difference spectra of the species, and not linear combinations of them as is the case with transient absorption SADS fitting. This model could be improved by accounting for the B-band inhomogeneity using a distribution of $\mathrm{B}_{A}$ and $\mathrm{B}_{B}$ peaks with homogeneous widths identified from the anti-diagonal cross-section of the B-band.

Testing models with branched charge separation pathways did not yield conclusive results. Showing that a given branched model agrees with our data is necessary, but not sufficient, to make a definitive assignment of a reaction scheme. We find that different branched charge separation models produce equally good fits to our data, and additional basis spectra beyond the eight used in the minimal model are linearly dependent on the others, suggesting that the information contained solely in the 2D spectrum from 700-900nm does not support the presence of branched pathways. In Section 4.3 we showed that the features in our data are consistent with several published transient absorption studies, which are essentially subsets of the 2D spectrum with lower excitation resolution. The non-uniqueness of the branched reaction schemes discussed in Section 1.2 helps to explain the lack of consensus and variety of proposed models in the literature. To better distinguish between these models, we require additional measurements which are more sensitive to specific charge separated states. Section 5.2 describes several potential methods to explore. 


\section{CHAPTER 5}

CONCLUSION

\subsection{Summary}

In this thesis, I have presented my contributions towards the ultimate goal of using 2DES to characterize the energy transfer and charge separation processes in bacterial reaction centers. From an experimental standpoint, this project has presented several technical challenges in extending our 2DES setup into the near IR wavelength range. The development of a three-stage degenerate optical parametric amplifier was a key component in obtaining high quality 2D spectra spanning the $\mathrm{P}, \mathrm{B}$ and $\mathrm{H}$ absorption bands of the $\mathrm{BRC}$ with $<15 \mathrm{fs}$ time resolution [159]. Using the DOPA, we have acquired 2DES scans of two BRC mutants, revealing excitation and polarization dependent population kinetics information on timescales ranging from $10 \mathrm{fs}$ to $1 \mathrm{~ns}$ [160]. Some additional experimental work not presented in this thesis includes preliminary data from 2-color 2DES using a NOPA probe to detect the 650nm Bchl and BPheo anion bands, and temperature-dependent 2DES on Bchl a and W(M250)V ranging from $5 \mathrm{~K}$ to $77 \mathrm{~K}$. I have also contributed to the development of the $2 \mathrm{D}$ Electronic Stark Spectroscopy (2DESS) experiment [144], working towards applying the technique on BRCs to better resolve signatures of charge separated states. These experiments will be discussed further in Section 5.2. Additionally, I worked on time-resolved second harmonic generation experiments, which measured photoinduced ultrafast charge transfer processes at the donor/acceptor interface of organic photovoltaic materials [161, 162].

After acquiring the 2DES data, the next challenge was to extract quantitatively meaningful information about the population kinetics. In Chapter 3, I presented an overview 
of the global and target analysis techniques commonly used for fitting transient absorption and 2DES data. The 2DDAS and 2DEAS approaches, whle useful for making qualitative observations about the time evolution of the $2 \mathrm{D}$ spectrum, proved to be inadequate for identifying branched and parallel reaction schemes. I implemented an improved 2D kinetic fitting method which uses information from the linear absorption spectrum and the BRC structure to model the excitation dependence of the time-resolved 2D spectrum. I developed a versatile 2D global fitting program for simultaneous excitation-dependent fitting of multiple data sets of different types, allowing for model-dependent constraints on specific basis spectra. The method employed by Thyrhaug et al. [67] and Dostal et al. [75], which was shown to uniquely characterize energy transfer kinetics in certain cases, is a special case of the method I describe in Chapter 3.

The results of the excitation-dependent global fitting on the $\mathrm{W}(\mathrm{M} 250) \mathrm{V}$ mutant showed that the entire time-dependent 2D spectrum is well-represented by a sequential reaction scheme in which photoexcitation of each chromophore leads to energy transfer to the special pair, followed by a single charge-separation pathway $\mathrm{P}^{*} \rightarrow \mathrm{P}^{+} \mathrm{B}_{A}^{-} \rightarrow \mathrm{P}^{+} \mathrm{H}_{A}^{-}$. The reconstructed 2D spectrum with this model uses only seven basis spectra which are shared between all excitation frequencies, some of which are constrained based on a comparison of spectral features shared between the two mutants. This global fitting approach reduces the number of free linear parameters by more than an order magnitude compared to 2DDAS or 2DEAS, with basis spectra corresponding to actual species associated spectra.

Our global analysis failed to reveal conclusive evidence for any of the alternative charge separation pathways described in Chapter 1. Despite the additional constraints imposed by the improved global fit, these branched kinetic models were not uniquely distinguishable. While we can choose a given branched model and show that it reproduces our data, we find that several such models reproduce the data equally well, and do not substantially improve the fit when compared to the unbranched model. This does not disprove the existence of these pathways, but it may cast some doubt on the uniqueness of some global fitting results 
from transient absorption studies purporting specific branched reaction schemes.

\section{$5.2 \quad$ Future Directions}

My contributions to the experimental setup and 2D global fitting have laid the groundwork for several future experiments which, combined with the 2DES data and global fitting methods described in this thesis, could provide a more complete picture of the energy landscape of the BRC which governs its ultrafast energy transfer and charge separation kinetics.

- Anion band probe. We have taken preliminary data with DOPA excitation of the P, $\mathrm{B}$ and $\mathrm{H}$ bands and a 600-700nm NOPA probe spanning the Bchl and BPheo anion bands. Probing this region can reveal direct signatures of the formation of the charge separated product states $[5,57]$, which could be added to the $2 \mathrm{D}$ global analysis to better distinguish between the proposed alternative charge separation pathways. The signal strength is very low in this region, making it challenging to obtain a sufficiently high signal-to-noise ratio to be suitable for global fitting. The signal-to-noise may be improved by performing transient grating experiments with narrower pump bandwidths and much longer averaging at each population time, rather than the full 2DES scan. The time resolution in this case should still be sufficient to resolve the picosecond charge separation kinetics. There is an additional Bchl anion band at 1020nm without an overlapping BPheo anion band [5]. Probing this region could isolate the population of $\mathrm{P}^{+} \mathrm{B}_{A}^{-}$from the BPheo anion states. A near-IR continuum probe could be used, or possibly a simple one or two-stage near-IR OPA copied from the current DOPA design.

- Polarization-dependent global fitting. We have 2D spectra from parallel, perpendicular, and magic angle pump/probe polarizations, but only the magic angle spectra were used in the global fitting presented here. Polarization control is a powerful tool for selectively exciting specific pigments and disentangling the signals from different pathways [57]. As discussed in Section 3.3, the parallel and perpendicular signals detected from each species are weighted by the relative transition dipole orientations of the initial and final 
state. To use the polarization components in the 2D global analysis, these weighting factors must be included, either as free parameters or from a priori knowledge. The dipole orientations of the initially excited states can be estimated from the crystal structure [16], but the charge separated product states present a challenge.

- $2 D$ and TG Stark Spectroscopy. We have recently demonstrated a method which extends the dimensionality of Stark spectroscopy using 2DES [144], which may be used to identify clearer spectral signatures of charge transfer states. In combination with the anion band experiments, 2DESS could help isolate the weak spectral components from intermediate charge separated states in the BRC.

- Low-temperature 2DES. We have also acquired preliminary 2D spectra of W(M250)V at 5K using a liquid helium-cooled cryostat. The effects of thermal broadening are further reduced compared to the $77 \mathrm{~K}$ spectrum, revealing the inhomogeneity of the B-band even more clearly. 2DES at low temperatures can also be used to better characterize the spectral densities for different electronic states [163] and refine simulations of 2D spectra based on excitonic models like the Novoderezhkin model for photosystem II $[81,82]$.

- Temperature-dependent kinetic fitting. The liquid helium cryostat enables precise temperature control from $5 \mathrm{~K}$ to room temperature. Adding the temperature dependence as another independent variable in the global fit could offer an additional constraint to uniquely identify specific kinetic components. A possible implementation of this is described in Section 3.1.3, and the 2D fitting program I developed can readily be adapted to model temperature-dependence.

- 2DES on additional BRC mutants. The direct comparison of W(M250)V and DLL kinetics proved useful for constraining the basis spectra of specific states. The discussion in Chapter 1 describes several other potentially interesting mutants to compare. In particular, the YM210W mutant shown to exhibit an alternate charge separation 
pathway $[35,53]$ would be useful for isolating basis spectra from charge intermeidate separated states.

- 2D fitting with inhomogeneity. The $2 \mathrm{D}$ global fit did not give physically reasonable B-band basis spectra due to the signficant inhomogeneous broadening of the B-band diagonal in the first few hundred femtoseconds. One might account for this by modeling the inhomogeneous $\mathrm{B}_{A}$ and $\mathrm{B}_{B}$ peaks with sets of narrow-bandwidth homogeneous states with a distribution of peak positions [75]. The transition rates from each inhomogeneous state might also need to be modeled with a distribution of rates $[62,78]$.

- Assessing generalizability of a model. One of the challenges inherent to global fitting is in determining how many components or free parameters to use. Adding more states to the model will always result in an improved residual, but beyond a certain point, the marginal improvement in residual comes from fitting random noise in the data rather than representing real trends. There are statistical methods which provide criteria for choosing a model, balancing the goodness of fit against model complexity [74]. The Bayesian Information Criterion, for instance, could be a useful tool for assessing the validity of a given branched reaction scheme $[164,165]$.

- 2DES on BRC crystals. Huang et al. have performed transient absorption measurements on single crystals of BRCs at cryogenic temperatures [57, 166], exploiting the fixed sample orientation to selectively excite $\mathrm{B}_{A}$ and $\mathrm{B}_{B}$ with different pump polarizations. Making BRC crystals of suitable optical quality for 2DES presents a challenge, but the the ability to precisely select excitation of A- and B- branch cofactos would help to further disentangle the spectral signatures from energy transfer and charge separation processes. 


\section{REFERENCES}

[1] Gregory D. Scholes, Graham R. Fleming, Alexandra Olaya-Castro, and Rienk van Grondelle. Lessons from nature about solar light harvesting. Nature Chemistry, 3(10): 763-774, 2011.

[2] Elisabet Romero, Vladimir I. Novoderezhkin, and Rienk van Grondelle. Quantum design of photosynthesis for bio-inspired solar-energy conversion. Nature, 543(7645): 355-365, 2017.

[3] A.J. Hoff and J. Deisenhofer. Photophysics of photosynthesis. Structure and spectroscopy of reaction centers of purple bacteria. Physics Reports, 287:1-247, 1997.

[4] Herbert Van Amerongen, Rienk Van Grondelle, and Leonas Valkunas. Photosynthetic excitons. World Scientific, 2000.

[5] Wolfgang Zinth and Josef Wachtveitl. The first picoseconds in bacterial photosynthesis - Ultrafast electron transfer for the efficient conversion of light energy. ChemPhysChem, 6(5):871-880, 2005.

[6] Robert E Blankenship. Molecular mechanisms of photosynthesis. John Wiley \& Sons, 2013.

[7] David L Nelson, Albert L Lehninger, and Michael M Cox. Lehninger principles of biochemistry. Macmillan, 2008.

[8] Graham R. Fleming and Rienk van Grondelle. The Primary Steps of Photosynthesis. Physics Today, 1994.

[9] J.P. Allen and J.C. Williams. Photosynthetic reaction centers. FEBS Letters, 438(1-2): 5-9, 1998.

[10] J Deisenhofer, O Epp, K Miki, R Huber, and H Michel. X-ray structure analysis of a membrane protein complex. Electron density map at $3 \mathrm{~A}$ resolution and a model of the chromophores of the photosynthetic reaction center from Rhodopseudomonas viridis. Journal of molecular biology, 180:385-398, 1984. 
[11] Johann Deisenhofer, Epp, Miki, Huber, and Michel. Structure of the protein subunits in the photosynthetic reaction centre of Rhodopseudomonas viridis at $3 \mathrm{~A}$ resolution. Nature, 318:618-624, 1985.

[12] C. H. Chang, D. Tiede, J. Tang, U. Smith, J. Norris, and M. Schiffer. Structure of Rhodopseudomonas sphaeroides R-26 reaction center. FEBS Letters, 205(1):82-86, 1986.

[13] T O Yeates, H Komiya, A Chirino, D C Rees, J P Allen, and G Feher. Structure of the reaction center from Rhodobacter sphaeroides R-26 and 2.4.1: protein-cofactor (bacteriochlorophyll, bacteriopheophytin, and carotenoid) interactions. PNAS, 85(21): 7993-7997, 1988.

[14] U Ermler, G Fritzsch, S K Buchanan, and H Michel. Structure of the photosynthetic reaction centre from Rhodobacter sphaeroides at 2.65 A resolution: cofactors and protein-cofactor interactions. Structure (London, England : 1993), 2(10):925-936, 1994.

[15] Gergely Katona, Ulf Andreasson, Ehud M Landau, Lars-Erik Andreasson, and Richard Neutze. Lipidic cubic phase crystal structure of the photosynthetic reaction centre from rhodobacter sphaeroides at 2.35 å resolution. Journal of molecular biology, 331 (3):681-692, 2003.

[16] Xanthipe J. Jordanides, Gregory D. Scholes, and Graham R. Fleming. The Mechanism of Energy Transfer in the Bacterial Photosynthetic Reaction Center. The Journal of Physical Chemistry B, 105(8):1652-1669, 2001.

[17] Y W Jia, T J Dimagno, C K Chan, Z Y Wang, M Du, D K Hanson, M Schiffer, J R Norris, G R Fleming, and M S Popov. Primary Charge Separation In Mutant Reaction Centers of Rhodobacter-capsulatus. J Phys Chem, 97(50):13180-13191, 1993.

[18] Ulrich Finkele, Christoph Lauterwasser, Wolfgang Zinth, Kevin A Gray, and Dieter Oesterhelt. Role of tyrosine M210 in the initial charge separation of reaction centers of Rhodobacter sphaeroides. Biochemistry, 29(1985):8517-8521, 1990.

[19] V. Nagarajan, W. Parson, D. Davis, and C. Schenck. Kinetics and Free Energy Gaps of Electron-Transfer Reactions in Rhodobacter sphaeroides Reaction Centers. Biochemistry, 32:12324-12336, 1993.

[20] W W Parson, Z T Chu, and a Warshel. Electrostatic control of charge separation in bacterial photosynthesis. Biochimica et biophysica acta, 1017(3):251-272, 1990. 
[21] B A Heller, D Holten, and C Kirmaier. Control of electron transfer between the Land M-sides of photosynthetic reaction centers. Science (New York, N.Y.), 269(5226): $940-5,1995$.

[22] Evaldas Katilius, Trieva Turanchik, Su Lin, Aileen K. W. Taguchi, and Neal W. Woodbury. B-Side Electron Transfer in a Rhodobacter sphaeroides Reaction Center Mutant in Which the B-Side Monomer Bacteriochlorophyll Is Replaced with Bacteriopheophytin. The Journal of Physical Chemistry B, 103(35):7386-7389, 1999.

[23] Philip D. Laible, Christine Kirmaier, C. S M Udawatte, Samuel J. Hofman, Dewey Holten, and Deborah K. Hanson. Quinone reduction via secondary B-branch electron transfer in mutant bacterial reaction centers. Biochemistry, 42(6):1718-1730, 2003.

[24] Christine Kirmaier, Philip D. Laible, Kazimierz Czarnecki, Aaron N. Hata, Deborah K. Hanson, David F. Bocian, and Dewey Holten. Comparison of M-side electron transfer in Rb. sphaeroides and Rb. capsulatus reaction centers. Journal of Physical Chemistry $B, 106(7): 1799-1808,2002$.

[25] S Lin, E Katilius, a L Haffa, a K Taguchi, and N W Woodbury. Blue light drives B-side electron transfer in bacterial photosynthetic reaction centers. Biochemistry, 40 (46):13767-73, 2001.

[26] M L Paddock, C. Chang, Q. Xu, E.C. Abresch, H.L. Axelrod, G. Feher, and M.Y. Okamura. Quinone (QB) Reduction by B-Branch Electron Transfer in Mutant Bacterial Reaction Centers from Rhodobacter sphaeroides : Quantum Efficiency and X-ray Structure. Biochemistry, 44:6920-6928, 2005.

[27] N W Woodbury, M Becker, D Middendorf, and W W Parson. Picosecond kinetics of the initial photochemical electron-transfer reaction in bacterial photosynthetic reaction centers. Biochemistry, 24(26):7516-21, 1985.

[28] C Kirmaier and D Holten. An assessment of the mechanism of inital electron transfer in bacterial reaction centers. Biochemistry, 30:609-613, 1991.

[29] N W Woodbury, J M Peloquin, R G Alden, X Lin, S Lin, a K Taguchi, J C Williams, and J P Allen. Relationship between thermodynamics and mechanism during photoinduced charge separation in reaction centers from Rhodobacter sphaeroides. Biochemistry, 33(26):8101-8112, 1994.

[30] a Ogrodnik, W Keupp, M Volk, G Aumeier, and Me Michel-Beyerle. Inhomogeneity of radical pair energies in photosynthetic reaction centers revealed by differences in recombination dynamics of $\mathrm{P}+\mathrm{HA}$-when detected in delayed Emission and in Absorption. J Phys Chem, 98:3432-3439, 1994. 
[31] Gilad Haran, Klaas Wynne, Chris C. Moser, P. Leslie Dutton, and Robin M. Hochstrasser. Level Mixing and Energy Redistribution in Bacterial Photosynthetic Reaction Centers. The Journal of Physical Chemistry, 100(13):5562-5569, 1996.

[32] Brett A. King, Tim B. McAnaney, Alex Dewinter, and Steven G. Boxer. Excited state energy transfer pathways in photosynthetic reaction centers. 3. Ultrafast emission from the monomeric bacteriochlorophylls. Journal of Physical Chemistry B, 104(37):8895$8902,2000$.

[33] RJ Stanley, Brett King, and SG Boxer. Excited state energy transfer pathways in photosynthetic reaction centers. 1. Structural symmetry effects. The Journal of Physical Chemistry, 3654(96):12052-12059, 1996.

[34] ME Van Brederode, MR Jones, and R Van Grondelle. Fluorescence excitation spectra of membrane-bound photosynthetic reaction centers of Rhodobacter sphaeroides in which the tyrosine M210 residue is replaced by tryptophan: evidence for a new pathway of charge separation. Chem. Phys. Lett., 268:143-149, 1997.

[35] Marion E Van Brederode, Frank Van Mourik, Ivo H M Van Stokkum, and Michael R Jones. Multiple pathways for ultrafast transduction of light energy in the photosynthetic reaction center of Rhodobacter sphaeroides. PNAS, 96(5):2054-9., 1999.

[36] Marten H Vos, Jean-Christophe Lambry, Steven J Robles, Douglas C Youvan, Jacques Breton, and Jean-Louis Martin. Femtosecond spectral evolution of the excited states of bacterial reaction centers at $10 \mathrm{~K}$. PNAS, 89:613-617, 1992.

[37] J Breton, J.-L. Martin, J Petrich, A Migus, and A Antonetti. The absence of a spectroscopically resolved intermediate state $\mathrm{P}+\mathrm{B}$ - in bacterial photosynthesis. FEBS Lett., 209(1):37-43, 1986.

[38] C K Chan, T J Dimagno, L X Q Chen, J R Norris, and G R Fleming. Mechanism of the Initial Charge Separation in Bacterial Photosynthetic Reaction Centers. PNAS, 88(24):11202-11206, 1991.

[39] J Breton, J L Martin, G R Fleming, and J C Lambry. Low-Temperature Femtosecond Spectroscopy of the Initial Step of Electron-Transfer in Reaction Centers From Photosynthetic Purple Bacteria. Biochemistry, 27(21):8276-8284, 1988.

[40] S. Schmidt, T. Arlt, P. Hamm, C. Lauterwasser, U. Finkele, G. Drews, and W. Zinth. Time-resolved spectroscopy of the primary photosynthetic processes of membranebound reaction centers from an antenna-deficient mutant of Rhodobacter capsulatus. BBA - Bioenergetics, 1144(3):385-390, 1993. 
[41] U Eberl, M Gilbert, W Keupp, T Langenbacher, J Siegl, I Sinning, A Ogrodnik, SJ Robles, J Breton, DC Youvan, et al. Fast internal conversion in bacteriochlorophyll dimers. In The Photosynthetic Bacterial Reaction Center II, pages 253-260. Springer, 1992.

[42] C Kirmaier and D Holten. Primary photochemistry of reaction centers from the photosynthetic purple bacteria. Photosynthesis research, 13(3):225-60, 1987.

[43] William W. Parson and Richard J. Cogdell. The primary photochemical reaction of bacterial photosynthesis. BBA Reviews On Bioenergetics, 416(1):105-149, 1975.

[44] R K Clayton. Primary Processes in Bacterial Photosynthesis. Annual Reviews, 2(1): 421-448, 1973.

[45] A Warshel, S Creighton, and W W Parson. Electron-Transfer Pathways in the Primary Event of Bacterial Photosynthesis. J. Phys. Chem., 92(1):2696-2701, 1988.

[46] P O J Scherer and Sighart F Fischer. Quantum treatment of the optical spectra and the initial electron transfer process within the reaction center of rhodopseudomonas viridis. Chemical Physics, 131:115-127, 1989.

[47] Sighart F Fischer and P O J Scherer. On the early charge separation and recombination processes in bacterial reaction centers. Chem. Phys., 115(2):151-158, 1987.

[48] W Holzapfel, U Finkele, W Kaiser, D Oesterhelt, H Scheer, H U Stilz, and W Zinth. Initial electron-transfer in the reaction center from Rhodobacter sphaeroides. PNAS, 87(13):5168-72, 1990.

[49] a. R. Holzwarth and Marc G. Müller. Energetics and kinetics of radical pairs in reaction centers from Rhodobacter sphaeroides. A femtosecond transient absorption study. Biochemistry, 35(96):11820-11831, 1996.

[50] J T M Kennis, A Y Shkuropatov, I H M van Stokkum, P Gast, A J Hoff, V A Shuvalov, and T J Aartsma. Formation of a long-lived $\mathrm{P}+\mathrm{B}(\mathrm{A})$ - state in plant Pheophytinexchanged reaction centers of Rhodobacter sphaeroides R26 at low temperature. Biochemistry, 36(97):16231-16238, 1997.

[51] S Lin, J Jackson, A K W Taguchi, and N W Woodbury. Excitation wavelength dependent spectral evolution in Rhodobacter sphaeroides R-26 reaction centers at low temperatures: The Q(y) transition region. Journal of Physical Chemistry B, 102(98): 4016-4022, 1998. 
[52] M E van Brederode, JP Ridge, Ivo H M van Stokkum, F van Mourik, MR Jones, and $R$ van Grondelle. On the efficiency of energy transfer and the different pathways of electron transfer in mutant reaction centers of Rhodobacter sphaeroides. Photosynthesis Research, 55:141-146, 1998.

[53] Marion E Van Brederode, Michael R Jones, Frank Van Mourik, and Ivo H M Van Stokkum. A New Pathway for Transmembrane Electron Transfer in Photosynthetic Reaction Centers of Rhodobacter sphaeroides Not Involving the Excited Special Pair. Biochemistry, 36(23), 1997.

[54] Marten H Vos, Jacques Breton, and Jean-Louis Martin. Electronic Energy Transfer within the Hexamer Cofactor System of Bacterial Reaction Centers. The Journal of Physical Chemistry B, 101(47):9820-9832, 1997.

[55] Huilin Zhou and Steven G. Boxer. Probing Excited-State Electron Transfer by Resonance Stark Spectroscopy. 1. Experimental Results for Photosynthetic Reaction Centers. J. Phys. Chem. B, 102(45):9139-9147, 1998.

[56] Huilin Zhou and Steven G. Boxer. Probing Excited-State Electron Transfer by Resonance Stark Spectroscopy. 2. Theory and Application. J. Phys. Chem. B, 102(91489160):9148-9160, 1998.

[57] Libai Huang, Nina Ponomarenko, Gary P Wiederrecht, and David M Tiede. Cofactorspecific photochemical function resolved by ultrafast spectroscopy in photosynthetic reaction center crystals. PNAS, 109(13):4851-6, mar 2012.

[58] B A King, R J Stanley, and S G Boxer. Excited state energy transfer pathways in photosynthetic reaction centers .2. Heterodimer special pair. J. Phys. Chem. B, 101 (18):3644-3648, 1997.

[59] S J Robles, J Breton, and D C Youvan. Partial symmetrization of the photosynthetic reaction center. Science, 248:1402-1405, 1990.

[60] J Fajer, D C Brune, M S Davis, A Forman, and L D Spaulding. Primary charge separation in bacterial photosynthesis: oxidized chlorophylls and reduced pheophytin. PNAS, 72(12):4956-4960, 1975.

[61] David M Jonas, Matthew J Lang, Yutaka Nagasawa, Taiha Joo, and Graham R Fleming. PumpProbe Polarization Anisotropy Study of Femtosecond Energy Transfer within the Photosynthetic Reaction Center of Rhodobacter sphaeroides R26. Journal of Physical Chemistry, 100(30):12660-12673, 1996. 
[62] Heinz Huber, M. Meyer, H. Scheer, W. Zinth, and Josef Wachtveitl. Temperature dependence of the primary electron transfer reaction in pigment-modified bacterial reaction centers. Photosynthesis Research, 55(2-3):153-162, 1998.

[63] Rudolph A Marcus. On the theory of oxidation-reduction reactions involving electron transfer. The Journal of Chemical Physics, 24(5):966-978, 1956.

[64] Andrei K. Dioumaev. Evaluation of intrinsic chemical kinetics and transient product spectra from time-resolved spectroscopic data. Biophysical Chemistry, 67(1-3):1-25, 1997.

[65] Yuan Chung Cheng, Gregory S. Engel, and Graham R. Fleming. Elucidation of population and coherence dynamics using cross-peaks in two-dimensional electronic spectroscopy. Chemical Physics, 341(1-3):285-295, 2007.

[66] Gitt Panitchayangkoon, Dugan Hayes, Kelly a Fransted, Justin R Caram, Elad Harel, Jianzhong Wen, Robert E Blankenship, and Gregory S Engel. Long-lived quantum coherence in photosynthetic complexes at physiological temperature. PNAS, 107(29): 12766-70, 2010.

[67] Erling Thyrhaug, Karel Zidek, Jakub Dostal, David Bina, and Donatas Zigmantas. Exciton Structure and Energy Transfer in the Fenna-Matthews-Olson Complex. Journal of Physical Chemistry Letters, 7:1653-1660, 2016.

[68] Jeffrey a. Myers, Kristin L. M. Lewis, Franklin D. Fuller, Patrick F. Tekavec, Charles F. Yocum, and Jennifer P. Ogilvie. Two-Dimensional Electronic Spectroscopy of the D1-D2-cyt b559 Photosystem II Reaction Center Complex. The Journal of Physical Chemistry Letters, 1(19):2774-2780, oct 2010.

[69] Franklin D Fuller, Jie Pan, Andrius Gelzinis, Vytautas Butkus, S Seckin Senlik, Daniel E Wilcox, Charles F Yocum, Leonas Valkunas, Darius Abramavicius, and Jennifer P Ogilvie. Vibronic coherence in oxygenic photosynthesis. Nature chemistry, 6 (8):706-11, 2014.

[70] Kristin L. M. Lewis and Jennifer P. Ogilvie. Probing Photosynthetic Energy and Charge Transfer with Two-Dimensional Electronic Spectroscopy. The Journal of Physical Chemistry Letters, 3(4):503-510, feb 2012.

[71] V P Singh, M Westberg, C Wang, P D Dahlberg, T Gellen, a T Gardiner, R J Cogdell, and G S Engel. Towards quantification of vibronic coupling in photosynthetic antenna complexes. The Journal of chemical physics, 142(21):212446, jun 2015.

[72] J F Nagle. Solving complex photocycle kinetics. Theory and direct method. Biophysical journal, 59(2):476-487, 1991. 
[73] Ivo H.M. van Stokkum, Delmar S. Larsen, and Rienk van Grondelle. Global and target analysis of time-resolved spectra. Biochimica et Biophysica Acta (BBA) - Bioenergetics, 1657(2-3):82-104, 2004.

[74] C. Ruckebusch, M. Sliwa, P. Pernot, A. de Juan, and R. Tauler. Comprehensive data analysis of femtosecond transient absorption spectra: A review. Journal of Photochemistry and Photobiology C: Photochemistry Reviews, 13(1):1-27, 2012.

[75] Jakub Dostal, Benesova, and Brixner. Two-Dimensional Electronic Spectroscopy Can Fully Characterize the Population Transfer in Molecular Systems. Journal of Chemical Physics, 145(124312), 2016.

[76] G J Small, J M Hayes, and R J Silbey. The Question of Dispersive Kinetics for the Initial Phase of Charge Separation in Bacterial Reaction Centers. J.Phys.Chem., 96: 7499-7501, 1992.

[77] T Arlt, S Schmidt, W Kaiser, C Lauterwasser, M Meyer, H Scheer, and W Zinth. The accessory bacteriochlorophyll: a real electron carrier in primary photosynthesis. PNAS, 90(24):11757-11761, 1993.

[78] Zhiyu Wang, Robert M. Pearlstein, Yiwei Jia, Graham R. Fleming, and James R. Norris. Inhomogeneous electron transfer kinetics in reaction centers of bacterial photosynthesis. Chemical Physics, 176(2-3):421-425, 1993.

[79] Andrius Gelzinis, Leonas Valkunas, Franklin D Fuller, Jennifer P Ogilvie, Shaul Mukamel, and Darius Abramavicius. Tight-binding model of the photosystem II reaction center: application to two-dimensional electronic spectroscopy. New Journal of Physics, 15(7):075013, jul 2013.

[80] K L M Lewis, F D Fuller, J a Myers, C F Yocum, S Mukamel, D Abramavicius, and J P Ogilvie. Simulations of the two-dimensional electronic spectroscopy of the photosystem II reaction center. J. Phys. Chem. A, 117(1):34-41, jan 2013.

[81] Vladimir I Novoderezhkin, Elisabet Romero, Jan P Dekker, and Rienk van Grondelle. Multiple charge-separation pathways in photosystem ii: Modeling of transient absorption kinetics. ChemPhysChem, 12(3):681-688, 2011.

[82] Vladimir I Novoderezhkin, Elena G Andrizhiyevskaya, Jan P Dekker, and Rienk van Grondelle. Pathways and timescales of primary charge separation in the photosystem ii reaction center as revealed by a simultaneous fit of time-resolved fluorescence and transient absorption. Biophysical journal, 89(3):1464-1481, 2005. 
[83] Nancy Makri, Eunji Sim, Dmitrii E Makarov, and Maria Topaler. Long-time quantum simulation of the primary charge separation in bacterial photosynthesis. PNAS, 93(9): 3926-3931, 1996.

[84] Hong Xu, Ru-Bo Zhang, Shu-Hua Ma, Zheng-Wang Qu, Xing-Kang Zhang, and QiYuan Zhang. Theoretical studies on the mechanism of primary electron transfer in the photosynthetic reaction center of rhodobacter sphaeroides. Photosynthesis research, 74 (1):11-36, 2002.

[85] William W Parson and Arieh Warshel. Mechanism of charge separation in purple bacterial reaction centers. In The Purple Phototrophic Bacteria, pages 355-377. Springer, 2009 .

[86] Benjamin P Fingerhut and Shaul Mukamel. Resolving the electron transfer kinetics in the bacterial reaction center by pulse polarized 2-d photon echo spectroscopy. The Journal of Physical Chemistry Letters, 3(13):1798-1805, 2012.

[87] David M Jonas. Two-dimensional femtosecond spectroscopy. Annual review of physical chemistry, 54:425-63, jan 2003.

[88] Robert W Boyd. Nonlinear optics. In Handbook of Laser Technology and Applications (Three-Volume Set), pages 161-183. Taylor \& Francis, 2003.

[89] Shaul Mukamel. Principles of nonlinear optical spectroscopy. Number 6. Oxford University Press on Demand, 1999.

[90] Jennifer P. Ogilvie and Kevin J. Kubarych. Multidimensional Electronic and Vibrational Spectroscopy: An Ultrafast Probe of Molecular Relaxation and Reaction Dynamics, volume 57 of Advances In Atomic, Molecular, and Optical Physics. Elsevier, 2009. ISBN 9780123747990.

[91] Paul R Berman and Vladimir S Malinovsky. Principles of laser spectroscopy and quantum optics. Princeton University Press, 2010.

[92] M Khalil, N Demirdöven, and A Tokmakoff. Obtaining absorptive line shapes in twodimensional infrared vibrational correlation spectra. Physical review letters, 90(4): 047401, 2003.

[93] Sarah M Gallagher Faeder and David M Jonas. Two-Dimensional Electronic Correlation and Relaxation Spectra: Theory and Model Calculations. J. Phys. Chem. A, 103:10489-10505, 1999. 
[94] Franklin D Fuller, Daniel E Wilcox, and Jennifer P Ogilvie. Pulse shaping based twodimensional electronic spectroscopy in a background free geometry. Optics Express, 22(1):17420-17428, 2014.

[95] Sang-Hee Shim and Martin T Zanni. How to turn your pump-probe instrument into a multidimensional spectrometer: 2D IR and Vis spectroscopies via pulse shaping. Phys Chem Chem Phys, 11(5):748-61, feb 2010.

[96] Zhengyang Zhang, Kym Lewis Wells, Edward William James Hyland, and Howe-Siang Tan. Phase-cycling schemes for pumpprobe beam geometry two-dimensional electronic spectroscopy. Chemical Physics Letters, 550:156-161, oct 2012.

[97] Dorine Keusters and Howe-Siang Tan. Role of Pulse Phase and Direction in TwoDimensional Optical Spectroscopy. The Journal of Physical Chemistry A, 103(49): 10369-10380, dec 1999.

[98] L. Lepetit, G. Chériaux, and M. Joffre. Linear techniques of phase measurement by femtosecond spectral interferometry for applications in spectroscopy. Journal of the Optical Society of America B, 12(12):2467, 1995.

[99] Christophe Dorrer, Nadia Belabas, Jean-Pierre Likforman, and Manuel Joffre. Spectral resolution and sampling issues in Fourier-transform spectral interferometry. Journal of the Optical Society of America B, 17(10):1795, 2000.

[100] Franz Milota, Craig N Lincoln, and Jürgen Hauer. Precise phasing of 2D-electronic spectra in a fully non-collinear phase-matching geometry. Optics express, 21(13):1590411, jul 2013.

[101] Chu-kang Tang, Joann C Williams, Aileen K W Taguchi, James P Allen, and Neal W Woodbury. P + H A - Charge Recombination Reaction Rate Constant in Rhodobacter sphaeroides Reaction Centers Is Independent of the P / P + Midpoint Potential . Biochemistry, 38:8794-8799, 1999.

[102] S. G. Johnson, D. Tang, R. Jankowiak, J. M. Hayes, Gerald J. Small, and D. M. Tiede. Primary donor state mode structure and energy transfer in bacterial reaction centers. The Journal of Physical Chemistry, 94(15):5849-5855, 1990.

[103] Christine; Kirmaier, Dewey; Holten, and William W. Parson. Picosecondphotodichroism studies of the transient states in Rhodopseudomonas sphaeroides reaction centers at $5 \mathrm{~K}$. Effects of electron transfer on the six bacteriochlorin pigments. Biochimica et Biophysica Acta, 808:49-61, 1985. 
[104] L. M. P. Beekman, I. H. M. van Stokkum, R. Monshouwer, A. J. Rijnders, P. McGlynn, R. W. Visschers, M. R. Jones, and R. van Grondelle. Primary Electron Transfer in Membrane-Bound Reaction Centers with Mutations at the M210 Position. The Journal of Physical Chemistry, 100(17):7256-7268, 1996.

[105] L M Beekman, R W Visschers, R Monshouwer, M Heer-Dawson, T A Mattioli, P McGlynn, C N Hunter, B Robert, I H van Stokkum, and R van Grondelle. Time-resolved and steady-state spectroscopic analysis of membrane-bound reaction centers from Rhodobacter sphaeroides: comparisons with detergent-solubilized complexes. Biochemistry, 34(45):14712-21, 1995.

[106] D C Arnett, C C Moser, P L Dutton, and N F Scherer. The first events in photosynthesis: Electronic coupling and energy transfer dynamics in the photosynthetic reaction center from Rhodobacter sphaeroides. Journal of Physical Chemistry B, 103 (11):2014-2032, 1999.

[107] I H van Stokkum, L M Beekman, M R Jones, M E van Brederode, and R van Grondelle. Primary electron transfer kinetics in membrane-bound Rhodobacter sphaeroides reaction centers: a global and target analysis. Biochemistry, 36(38):11360-8, 1997.

[108] Neal W. Woodbury, Su Lin, Xiaomei Lin, Jeffrey M. Peloquin, Aileen K.W. Taguchi, JoAnn C. Williams, and James P. Allen. The role of reaction center excited state evolution during charge separation in a Rb. sphaeroides mutant with an initial electron donor midpoint potential $260 \mathrm{mV}$ above wild type. Chemical Physics, 197(3):405-421, aug 1995.

[109] C Kirmaier and D Holten. Evidence that a distribution of bacterial reaction centers underlies the temperature and detection-wavelength dependence of the rates of the primary electron-transfer reactions. PNAS, 87(9):3552-6, 1990.

[110] Marc G. Müller, Kai Griebenow, and Alfred R. Holzwarth. Primary processes in isolated bacterial reaction centers from Rhodobacter sphaeroides studied by picosecond fluorescence kinetics. Chemical Physics Letters, 199(5):465-469, 1992.

[111] J C Williams, R G Alden, H a Murchison, J M Peloquin, N W Woodbury, and J P Allen. Effects of mutations near the bacteriochlorophylls in reaction centers from Rhodobacter sphaeroides. Biochemistry, 31(45):11029-11037, 1992.

[112] S Lin, W Xiao, J E Eastman, A K W Taguchi, and N W Woodbury. Low-temperature femtosecond-resolution transient absorption spectroscopy of large-scale symmetry mutants of bacterial reaction centers. Biochemistry, 35:3187-3196, 1996. 
[113] Daniel E. Wilcox, Matthew E. Sykes, Andrew Niedringhaus, Max Shtein, and Jennifer P. Ogilvie. Heterodyne-detected and ultrafast time-resolved second-harmonic generation for sensitive measurements of charge transfer. Optics Letters, 39(14):4274, jul 2014.

[114] Vadim V Lozovoy, Igor Pastirk, and Marcos Dantus. Multiphoton intrapulse interference. IV. Ultrashort laser pulse spectral phase characterization and compensation. Optics Letters, 29(7):775, 2004.

[115] Brent Donovan, Larry a. Walker, Charles F. Yocum, and Roseanne J. Sension. Transient Absorption Studies of the Primary Charge Separation in Photosystem II. The Journal of Physical Chemistry, 100(5):1945-1949, 1996.

[116] S. Sporlein, W Zinth, M Meyer, H Scheer, and J Wachtveitl. Primary electron transfer in modified bacterial reaction centers: Optimization of the First Events in Photosynthesis. Chemical Physics Letters, 322:454-464, 2000.

[117] Alexander B. Doust, Ivo H M Van Stokkum, Delmar S. Larsen, Krystyna E. Wilk, Paul M G Curmi, Rienk Van Grondelle, and Gregory D. Scholes. Mediation of ultrafast lightharvesting by a central dimer in phycoerythrin 545 studied by transient absorption and global analysis. Journal of Physical Chemistry B, 109(29):14219-14226, 2005.

[118] Patrick F Tekavec, Jeffrey a Myers, Kristin L M Lewis, and Jennifer P Ogilvie. Twodimensional electronic spectroscopy with a continuum probe. Optics letters, 34(9): 1390-1392, 2009.

[119] M Siddiqui, G Cirmi, D Brida, F X Kärtner, and G Cerullo. Generation of 7 fs pulses at $800 \mathrm{~nm}$ from a blue-pumped optical parametric amplifier at degeneracy. Optics letters, 34(22):3592-4, nov 2009.

[120] T Wilhelm, J Piel, and E Riedle. Sub-20-fs pulses tunable across the visible from a blue-pumped single-pass noncollinear parametric converter. Optics letters, 22(19): 1494-6, oct 1997.

[121] Giulio Cerullo and Sandro De Silvestri. Ultrafast optical parametric amplifiers. Review of Scientific Instruments, 74(1):1, 2003.

[122] Amnon Yariv and Pochi Yeh. Optical waves in crystals, volume 10. Wiley, New York, 1984.

[123] David Eimerl, L Davis, S Velsko, EK Graham, and A Zalkin. Optical, mechanical, and thermal properties of barium borate. Journal of applied physics, 62(5):1968-1983, 1987. 
[124] A Holzwarth. Data analysis of time-resolved measurements. Biophysical techniques in photosynthesis, pages 75-92, 2004.

[125] E R Henry. The use of matrix methods in the modeling of spectroscopic data sets. Biophysical journal, 72:652-73, 1997.

[126] J K H Horber, W Gobel, A Ogrodnik, M E Michel-Beyerle, and R J Cogdell. Timeresolved measurements of fluorescence from reaction centers of Rhodopseudomonas sphaeroides R26.1. FEBS Lett., 198(2):273-278, 1986.

[127] Christine Kirmaier, Dewey Holten, and William W. Parson. Temperature and detection-wavelength dependence of the picosecond electron-transfer kinetics measured in Rhodopseudomonas sphaeroides reaction centers. Resolution of new spectral and kinetic components in the primary charge-separation process. Biochimica et Biophysica Acta (BBA) - Bioenergetics, 810(1):33-48, 1985.

[128] Luc Pronzato, Eric Walter, Alain Venot, and Jean Francois Lebruchec. A generalpurpose global optimizer: Implimentation and applications. Mathematics and Computers in Simulation, 26(5):412-422, 1984.

[129] Gene Golub and Victor Pereyra. Separable nonlinear least squares: the variable projection method and its applications. Inverse Problems, 19(2):R1-R26, 2003.

[130] K Madsen, H B Nielsen, and O Tingleff. Methods for non-linear least squares problems. Infomatics and Mathematical Modeling, 2:1-30, 2004.

[131] G.H. Golub and V. Pereyra. The differentiation of pseudo-inverses and nonlienar least squares problems whose variables separate. SIAM J. Numer. Anal., 10(2):413-432, 1973.

[132] A.L.M Haffa, S Lin, E Katilius, J. C. Williams, a. K. W. Taguchi, J. P. Allen, and N.W. Woodbury. The Dependence of the Initial Electron Transfer Rate on Driving Force in Rhodobacter sphaeroides Reaction Centers. J. Phys. Chem. B, 106:7376-7384, 2002.

[133] J F Nagle, L A Parodi, and R H Lozier. Procedure for testing kinetic models of the photocycle of bacteriorhodopsin. Biophysical journal, 38(2):161-74, 1982.

[134] John F Nagle, Laszlo Zimanyi, and Janos K Lanyi. Testing BR Photocycle Kinetics. Biophysical Journal, 68(4):1490-1499, 1995.

[135] Jeffrey M. Peloquin, Su Lin, Aileen K. W. Taguchi, and Neal W. Woodbury. Excitation Wavelength Dependence of Bacterial Reaction Center Photochemistry. 1. Ground State and Excited State Evolution. The Journal of Physical Chemistry, 99(4):1349-1356, 1995. 
[136] Natalia P Pawlowicz, Ivo H M van Stokkum, Jacques Breton, Rienk van Grondelle, and Michael R Jones. An investigation of slow charge separation in a tyrosine M210 to tryptophan mutant of the Rhodobacter sphaeroides reaction center by femtosecond mid-infrared spectroscopy. Phys Chem Chem Phys, 12(11):2693-705, 2010.

[137] Elisabet Romero, Ivo H M Van Stokkum, Vladimir I. Novoderezhkin, Jan P. Dekker, and Rienk Van Grondelle. Two different charge separation pathways in photosystem II. Biochemistry, 49(20):4300-4307, 2010.

[138] Andrius Gelzinis, Vytautas Butkus, Egidijus Songaila, Ramunas Augulis, Andrew Gall, Claudia Büchel, Bruno Robert, Darius Abramavicius, Donatas Zigmantas, and Leonas Valkunas. Mapping energy transfer channels in fucoxanthin-chlorophyll protein complex. Biochimica et Biophysica Acta - Bioenergetics, 1847(2):241-247, 2015.

[139] Jan Alster, Heiko Lokstein, Jakub Dostál, Akira Uchida, and Donatas Zigmantas. Spectroscopic Study of Water Soluble Chlorophyll-Binding Protein from Lepidium Virginicum. The Journal of Physical Chemistry B, 118:3524-3531, 2014.

[140] Margherita Maiuri, Julien Réhault, Anne Marie Carey, Kirsty Hacking, Marco Garavelli, Larry Lüer, Dario Polli, Richard J. Cogdell, and Giulio Cerullo. Ultra-broadband 2D electronic spectroscopy of carotenoid-bacteriochlorophyll interactions in the LH1 complex of a purple bacterium. Journal of Chemical Physics, 142(21), 2015.

[141] Evgeny E. Ostroumov, Rachel M. Mulvaney, Jessica M. Anna, Richard J. Cogdell, and Gregory D. Scholes. Energy transfer pathways in light-harvesting complexes of purple bacteria as revealed by global kinetic analysis of two-dimensional transient spectra. Journal of Physical Chemistry B, 117(38):11349-11362, 2013.

[142] Evgeny E Ostroumov, Rachel M Mulvaney, Richard J Cogdell, and Gregory D Scholes. Broadband 2D electronic spectroscopy reveals a carotenoid dark state in purple bacteria. Science, 340(6128):52-6, 2013.

[143] A Ndrea V Olpato, L U C A B Olzonello, E Lena M Eneghin, and E Lisabetta C Ollini. Global analysis of coherence and population dynamics in 2D electronic spectroscopy. Optics Express, 24(21):24773-24785, 2016.

[144] Anton Loukianov, Andrew Niedringhaus, Brandon Berg, Jie Pan, S. Seckin Senlik, and Jennifer P. Ogilvie. Two-dimensional Electronic Stark Spectroscopy. The Journal of Physical Chemistry Letters, 8:679-683, 2017.

[145] Sebastian Schott, Andreas Steinbacher, Johannes Buback, Patrick Nuernberger, and Tobias Brixner. Generalized magic angle for time-resolved spectroscopy with laser pulses of arbitrary ellipticity. Journal of Physics B: Atomic, Molecular and Optical Physics, 47(12):124014, 2014. 
[146] Jennifer P. Ogilvie, Marie Plazanet, Gami Dadusc, and R. J. Dwayne Miller. Dynamics of Ligand Escape in Myoglobin: Q-Band Transient Absorption and Four-Wave Mixing Studies. The Journal of Physical Chemistry B, 106(40):10460-10467, oct 2002.

[147] Robin M. Hochstrasser. Two-dimensional IR-spectroscopy: Polarization anisotropy effects. Chemical Physics, 266(2-3):273-284, 2001.

[148] Martin T Zanni, N H Ge, Yung Sam Kim, and Robin M Hochstrasser. Two-dimensional IR spectroscopy can be designed to eliminate the diagonal peaks and expose only the crosspeaks needed for structure determination. PNAS, 98(20):11265-11270, 2001.

[149] Andreas C. Albrecht. Polarizations and assignments of transitions: The method of photoselection. J. Mol. Spectrosc., 6:84-108, 1961.

[150] S Schmidt, T Arlt, P Hamm, H Huber, T Nägele, J Wachtveitl, W Zinth, M Meyer, and $\mathrm{H}$ Scheer. Primary electron-transfer dynamics in modified bacterial reaction centers containing pheophytin-a instead of bacteriopheophytin-a. Spectrochimica Acta Part A: Molecular and Biomolecular Spectroscopy, 51(9):1565-1578, 1995.

[151] S.H. Lin, M. Hayashi, S. Suzuki, X. Gu, W. Xiao, and M. Sugawara. Theoretical analyses on femtosecond time-resolved spectra of initial electron transfer of photosynthetic reaction centers at low temperatures. Chemical Physics, 197(3):435-455, aug 1995.

[152] Haberle, Lossau, Friese, Hartwich, A Ogrodnikl, H Scheer, and M.E. Michel-Beyerle. Ultrafast Electron and Exitation Energy Transfer in Modified Photosynthetic Reaction Centers from Rhodobacter sphaeroides. The Reaction Center of Photosynthetic Bacteria, 1996.

[153] Su Lin, Aileen K W Taguchi, and Neal W Woodbury. Excitation wavelength dependence of energy transfer and charge separation in reaction centers from Rhodobacter sphaeroides: Evidence for adiabatic electron transfer. J. Phys. Chem., 100(42):1706717078, 1996.

[154] Thomas F. Coleman and Yuying Li. On the convergence of interior-reflective Newton methods for nonlinear minimization subject to bounds. Mathematical Programming, 67(1-3):189-224, 1994.

[155] Thomas F. Coleman and Yuying Li. An Interior Trust Region Approach for Nonlinear Minimization Subject to Bounds. SIAM Journal on Optimization, 6(2):418-445, 1996.

[156] James; Kennedy and Russell; Eberhart. Particle swarm optimization. Encyclopedia of Machine Learning, pages 1942-1948, 1995. 
[157] Michael K Yetzbacher, Nadia Belabas, Katherine a Kitney, and David M Jonas. Propagation, beam geometry, and detection distortions of peak shapes in two-dimensional Fourier transform spectra. The Journal of chemical physics, 126:044511, jan 2007.

[158] J L Martin, J Breton, a J Hoff, A Migus, and A Antonetti. Femtosecond spectroscopy of electron transfer in the reaction center of the photosynthetic bacterium Rhodopseudomonas sphaeroides R-26: Direct electron transfer from the dimeric bacteriochlorophyll primary donor to the bacteriopheophytin acceptor with a t. PNAS, 83(4):957-61, 1986.

[159] Andrew Niedringhaus, Veronica R Policht, and Jennifer P Ogilvie. Probing ultrafast dynamics of bacteriochlorophyll-a using pulse shaping based $2 \mathrm{~d}$ electronic spectrometer with a degenerate opa. In 2016 Conference on Lasers and Electro-Optics (CLEO). IEEE, 2016.

[160] Andrew J Niedringhaus, Veronica R Policht, and Jennifer P Ogilvie. Probing ultrafast dynamics of bacterial reaction centers using two-dimensional electronic spectroscopy. In International Conference on Ultrafast Phenomena, volume UM4A-4. Optical Society of America, 2016.

[161] Daniel E Wilcox, Matthew E Sykes, Andrew Niedringhaus, Max Shtein, and Jennifer P Ogilvie. Heterodyne-detected and ultrafast time-resolved second-harmonic generation for sensitive measurements of charge transfer. Optics letters, 39(14):4274-4277, 2014.

[162] Daniel E Wilcox, Myeong H Lee, Matthew E Sykes, Andrew Niedringhaus, Eitan Geva, Barry D Dunietz, Max Shtein, and Jennifer P Ogilvie. Ultrafast charge-transfer dynamics at the boron subphthalocyanine chloride/c60 heterojunction: Comparison between experiment and theory. The journal of physical chemistry letters, 6(3):569$575,2015$.

[163] Olga Rancova, Ryszard Jankowiak, and Darius Abramavicius. Probing environment fluctuations by two-dimensional electronic spectroscopy of molecular systems at temperatures below $5 \mathrm{~K}$. The Journal of chemical physics, 142(21):212428, jun 2015.

[164] Jay I Myung and Mark A Pitt. Model comparison methods. Methods in Enzymology, 383:351-366, 2004.

[165] Jay I Myung, Yun Tang, and Mark A Pitt. Evaluation and comparison of computational models. Methods in Enzymology, 454:287-304, 2009.

[166] Libai Huang, Gary P Wiederrecht, Lisa M Utschig, Sandra L Schlesselman, Christina Xydis, Philip D Laible, Deborah K Hanson, and David M Tiede. Correlating ultrafast function with structure in single crystals of the photosynthetic reaction center. Biochemistry, 47(44):11387-11389, 2008. 